\title{
Asymptotic inference for linear stochastic differential equations with time delay
}

\author{
Ph.D. Thesis \\ by \\ JÁnos Marcell Benke
}

Supervisor: Professor Gyula Pap

Doctoral School of Mathematics and Computer Science Bolyai Institute

University of Szeged, Faculty of Science and Informatics

Szeged, 2018 



\section{Contents}

1 Introduction $\quad 1$

1.1 Some motivational applications for delayed models . . . . . . . . . . . . . . 1

1.2 Local asymptotic normality . . . . . . . . . . . . . . . . . . 4

1.3 Historical background . . . . . . . . . . . . . . . . . . . . 6

2 Asymptotic statistics $\quad 9$

2.1 Local asymptotic properties of statistical experiments . . . . . . . . . . . . 9

2.2 Consequences of the local asymptotic properties . . . . . . . . . . . . . . 14

2.3 An example for a Heston model . . . . . . . . . . . . . . . . . . 16

2.3.1 A short introduction and preliminaries . . . . . . . . . . 16

2.3 .2 Radon-Nikodym derivatives for Heston models . . . . . . . . . . . . . . . 19

2.3 .3 Subcritical case . . . . . . . . . . . . . . . . . . . . . 22

2.3 .4 Critical case . . . . . . . . . . . . . . . . . . 25

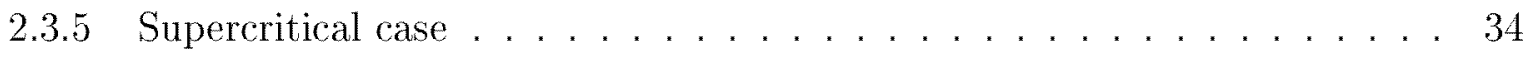

3 Statistical models for linear stochastic differential equation with time delay 39

3.1 Preliminaries for linear stochastic differential equations with time delay . . . . . 39

3.2 The special case of uniformly distributed time delay . . . . . . . . . . . . . . 42

3.2 .1 Radon-Nikodym derivatives . . . . . . . . . . . . . . . 43

3.2 .2 Local asymptotics of the likelihood ratios . . . . . . . . . . . . . 44

3.2 .3 Maximum likelihood estimates . . . . . . . . . . . . . . 45

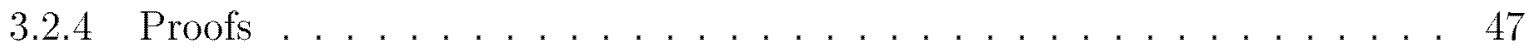

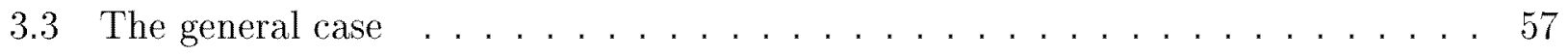

3.3 .1 Radon-Nikodym derivatives $\ldots \ldots \ldots \ldots$ 
3.3 .2 Local asymptotics of the likelihood ratios . . . . . . . . . . . 58

3.3 .3 Maximum likelihood estimates . . . . . . . . . . . . 62

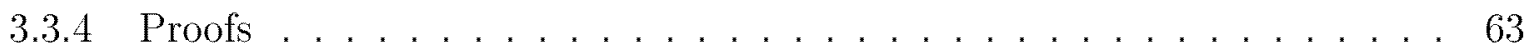

$\begin{array}{lr}\text { A Summary } & 79\end{array}$

$\begin{array}{lr}\text { B Összefoglaló } & 83\end{array}$

$\begin{array}{lr}\text { Bibliography } & 87\end{array}$ 


\section{Köszönetnyilvánítás}

Mindenek előtt köszönetet szeretnénk mondani témavezetőmnek, Dr. Pap Gyulának, aki MSc hallgató koromban bevezetett a sztochasztikus jelenségek világába, gondos irányítással egyengette doktoranduszi tanulmányaimat, és aki nélkül ez az értekezés nem születhetett volna meg.

Természetesen más utat jártam volna be, ha nem Dr. Krisztin Tibor lett volna a BSc szakdolgozatom témavezetôje és nem ismertet meg engem a dinamikus rendszerek matematikájával. Ez úton is köszönöm neki. Rajtuk kívül köszönettel tartozom a Bolyai Intézet egészének, mivel remek keretet adott a tanulmányaim és munkám végzéséhez.

A legnagyobb köszönet a családomat és ezen belül a szüleimet illeti, akik mindig mellettem álltak és állnak, valamint támogatták, hogy az legyek, aki szeretnék. Köszönöm! 



\section{Notation}

\begin{tabular}{|c|c|}
\hline $\mathbb{N}$ & set of positive integers \\
\hline $\mathbb{Z}_{+}$ & set of non-negative integers \\
\hline $\mathbb{R}$ & set of real numbers \\
\hline $\mathbb{R}_{+}$ & set of non-negative real numbers \\
\hline $\mathbb{R}_{++}$ & set of positive real numbers \\
\hline $\mathbb{R}_{-}$ & set of non-positive real numbers \\
\hline $\mathbb{R}_{--}$ & set of negative real numbers \\
\hline $\mathbb{C}$ & set of complex numbers \\
\hline$x \wedge y$ & $\min (x, y)$ for $x, y \in \mathbb{R}$ \\
\hline$x \vee y$ & $\max (x, y)$ for $x, y \in \mathbb{R}$ \\
\hline$\|x\|$ & Euclidean norm of a vector $\boldsymbol{x} \in \mathbb{R}^{d}$ \\
\hline$\|\boldsymbol{A}\|$ & induced matrix norm of a matrix $\boldsymbol{A} \in \mathbb{R}^{d \times d}$ \\
\hline$A \otimes B$ & Kronecker product of matrices $\boldsymbol{A}$ and $\boldsymbol{B}$ \\
\hline $\boldsymbol{I}_{d}$ & the $d$-dimensional unit matrix \\
\hline$\stackrel{\mathbb{P}}{\longrightarrow}$ & convergence in probability \\
\hline$\stackrel{\mathcal{D}}{\longrightarrow}$ & convergence in distribution \\
\hline$\stackrel{\text { a.s. }}{\longrightarrow}$ & convergence almost surely \\
\hline$\Rightarrow$ & convergence of the finite dimensional distributions \\
\hline$C\left(I, \mathbb{R}^{d}\right)$ & $\begin{array}{l}\text { the Banach space of continuous functions from the interval } I \subseteq \mathbb{R} \\
\text { to the set } \mathbb{R}^{d}, d \in \mathbb{N} \text { with the supremum norm }\end{array}$ \\
\hline $\mathcal{B}(X)$ & the Borel $\sigma$-algebra on a topological space $X$ \\
\hline $\operatorname{Res}_{z=\lambda}(f(z))$ & residue of the function $f$ at $\lambda$ \\
\hline LAQ & local asymptotic quadratic, see Definition 2.1.4 \\
\hline LAMN & local asymptotic mixed normal, see Definition 2.1 .5 \\
\hline LAN & local asymptotic normal, see Definition 2.1 .6 \\
\hline PLAMN & periodic local asymptotic mixed normal, see Definition 2.1 .7 \\
\hline MLE & maximum likelihood estimator \\
\hline $\mathcal{N}\left(\mu, \sigma^{2}\right)$ & $\begin{array}{l}\text { the normal (or Gaussian) distribution with expected value } \mu \in \mathbb{R} \\
\text { and variance } \sigma^{2} \in \mathbb{R}_{++}\end{array}$ \\
\hline $\mathcal{N}_{d}(\boldsymbol{\mu}, \boldsymbol{\Sigma})$ & $\begin{array}{l}\text { the } d \text {-dimensional normal (or Gaussian) distribution with expected } \\
\text { value } \boldsymbol{\mu} \in \mathbb{R}^{d} \text { and covariance matrix } \Sigma \in \mathbb{R}^{d \times d}\end{array}$ \\
\hline
\end{tabular}





\section{Chapter 1}

\section{Introduction}

In this thesis the local asymptotic properties of time delayed linear stochastic differential equations are studied. This section is intended to explain, why this topic was chosen for mathematical investigation. In Section 1.1 some time delayed models are presented to emphasize how useful and applicable are these type of models in many area. In Section 1.2 we introduce the local asymptotic normality, which is the basic concept of asymptotic statistics. Finally, in Section 1.3 we give an overview of the papers concerning results of this topic.

\subsection{Some motivational applications for delayed models}

Many dynamical systems can be well described with deterministic differential equations. However in many cases, it would be desirable to raise the complexity of our model by using time delay. Indeed, in many real world phenomena time delay or feedback occurs in the dynamics. An illustrative example is the logistic equation, which is given by

$$
\frac{\mathrm{d} N(t)}{\mathrm{d} t}=r N(t)\left(1-\frac{N(t)}{K}\right),
$$

where $N(t)$ is the size of a population at time $t, r$ and $K$ are the growth rate and the carrying capacity of the population, see, e.g., Erneux [15]. This model describes the population dynamics well in the case, when the underlying organisms' birth and/or death rates respond instantaneously to changes in population size. However, there are organisms that exhibit pulses of reproduction and have some lag time before they reproduce again. Thus time delay occurs in the dynamics. Hutchinson introduced the following so-called delayed logistic equation or Hutchinson's equation

$$
\frac{\mathrm{d} N(t)}{\mathrm{d} t}=r N(t)\left(1-\frac{N(t-\tau)}{K}\right),
$$

where $\tau$ is the time that is required for an individual to be able to reproduce. 
The so-called Wright's equation is defined by

$$
\frac{\mathrm{d} x(t)}{\mathrm{d} t}=-\alpha\left(\mathrm{e}^{x(t-1)}-1\right)
$$

which is connected to the density of prime numbers (see Wright [47]) is in strong relationship with the delayed logistic equation. Although this is one of the simplest but nonlinear delay differential equation, it has been researched intensively, see, e.g., Bánhelyi et al. [4].

Time delay occurs in many other fields, e.g., biology, chemistry, physics, (see, e.g., Erneux [15]), economics (see, e.g., Liz and Röst [34]) or in epidemics (see, e.g., Röst and Wu [43]).

If we would like to make our model more complex and hence more precise, we can consider stochastic delay differential equations. Indeed, many dynamical systems are difficult to describe accurately or it is impossible with deterministic setup. This is the case especially in financial applications. A good example for this the following, which was presented in the paper by Appleby, Riedle and Swords [2]. They studied the asymptotic behaviour of an stochastic delay differential equation modeling the evolution of the cumulative return of a risky security. In the model, the traders of the security determine their investment strategy by comparing short- and long-run moving averages of the security's returns.

The following results can be founded in Appleby, Riedle and Swords [2]. Let $(S(t))_{t \geq 0}$ denote the prices of a risky asset which satisfy

$$
\mathrm{d} S(t)=S(t) \mathrm{d} R(t) \quad t \geq 0
$$

where $R(t)$ denotes the cumulative return at time $t$. We assume that the cumulative returns $R$ follow a linear trend $\mu$. Suppose that there are $N$ traders in the economy, who determine their demand based on the cumulative de-trended returns $Y(t):=R(t)-\mu t$ on the asset. The trading strategy of the $j$-th agent at time $t$ is as follows: they consider a short-run moving average of the cumulative de-trended returns price over the last $\theta_{j}$ units of time

$$
\int_{\left[-\theta_{j}, 0\right]} Y(t+u) s_{j}(\mathrm{~d} u),
$$

where $s_{j}$ is a signed Borel-measure on $\left[-\theta_{j}, 0\right]$, and also calculate a long-run average of cumulative de-trended returns over the last $\tau_{j} \geq \theta_{j}$ units of time

$$
\int_{\left[-\tau_{j}, 0\right]} Y(t+u) l_{j}(\mathrm{~d} u),
$$

where $l_{j}$ is a signed Borel-measure on $\left[-\tau_{j}, 0\right]$. The measures $s_{j}$ and $l_{j}$ reflect the weights the agent puts on the different past values. In order to make the short-run and long-run comparable the measures $s_{j}$ and $l_{j}$ are chosen such that

$$
s_{j}\left(\left[-\theta_{j}, 0\right]\right)=l_{j}\left(\left[-\tau_{j}, 0\right]\right)
$$

We extend $s_{j}$ to a Borel-measure on $\left[-\tau_{j}, 0\right]$ by setting $s_{j}(I)=0$ for any Borel set $I \subseteq\left[-\tau_{j},-\theta_{j}\right)$. These averages can be distinguished as being "short-run" and "long-run" by 
hypothesising that the short-run average always allocates at least as much weights to the most recent $t$ time units of return as the long-run average does. Mathematically, this means that

$$
s_{j}([-t, 0]) \geq l_{j}([-t, 0]) \quad \text { for all } t \in\left[0, \tau_{j}\right] .
$$

The averages are distinguishable by presuming that $s_{j} \neq l_{j}$.

Trader $j$ then has planned demand at time $t$, which depends upon the strength of the signal received from the market, the signal being stronger the greater the difference between the short-run an long-run average. We assume that the trader buys the asset if the short-run average exceeds the long-run average and they sell the asset if the short-run average lies below the long-run average. The planned excess demand of trader $j$ at time $t$ is

$$
\beta_{j}\left(\int_{\left[-\theta_{j}, 0\right]} Y(t+u) s_{j}(\mathrm{~d} u)-\int_{\left[-\tau_{j}, 0\right]} Y(t+u) l_{j}(\mathrm{~d} u)\right),
$$

where $\beta_{j} \geq 0$. Therefore, the overall planned excess demand of all $N$ traders is

$$
\sum_{j=1}^{N} \beta_{j}\left(\int_{\left[-\theta_{j}, 0\right]} Y(t+u) s_{j}(\mathrm{~d} u)-\int_{\left[-\tau_{j}, 0\right]} Y(t+u) l_{j}(\mathrm{~d} u)\right) .
$$

The constants $\beta_{j}$ model the different influence of each trader on the total excess demand. Speculators react to other random stimuli - "news" - which are independent of past returns. The increments of this news are independent, so if the stimulus is a continuous process, this may be thought of as adding a further $\sigma\left(W\left(t_{2}\right)-W\left(t_{1}\right)\right)$ to the traders' excess demand over the time intervall $\left[t_{1}, t_{2}\right]$, where $W$ is a Brownian motion and $\sigma \geq 0$.

Finally, we suppose that the de-trended returns increase when there is excess demand, with the rise being larger the greater the excess demand. One way to capture this is to suppose that the evolution of the de-trended returns is described by

$$
\mathrm{d} Y(t)=\sum_{j=1}^{N} \beta_{j}\left(\int_{\left[-\theta_{j}, 0\right]} Y(t+u) s_{j}(\mathrm{~d} u)-\int_{\left[-\tau_{j}, 0\right]} Y(t+u) l_{j}(\mathrm{~d} u)\right) \mathrm{d} t+\sigma \mathrm{d} W(t) .
$$

We extend all measures $s_{j}$ and $l_{j}$ to the interval $[-\tau, 0]$, where $\tau=\max \left\{\tau_{1}, \ldots, \tau_{N}\right\}$ by setting them to zero outside their support. By introducing the Borel-measure $\nu$ on $[-\tau, 0]$ defined by

$$
\nu(\mathrm{d} u)=\sum_{j=1}^{N} \beta_{j}\left(s_{j}-l_{j}\right)(\mathrm{d} u)
$$

and the linear functional $L$ defined by

$$
L: C([-\tau, 0], \mathbb{R}) \rightarrow \mathbb{R}, \quad L \phi=\int_{[-\tau, 0]} \phi(u) \nu(\mathrm{d} u),
$$

we can rewrite Equation (1.1.4) as

$$
\mathrm{d} Y(t)=L\left(Y_{t}\right) \mathrm{d} t+\sigma \mathrm{d} W(t), \quad t \geq 0,
$$


where $Y_{t}$ is the segment of $Y$ at time $t$, namely $Y_{t}(u)=Y(t+u)$, where $u \in[-\tau, 0]$.

Because of (1.1.3), the evolution of the price of the risky asset $(S(t))_{t \geq 0}$ is now given by

$$
\mathrm{d} S(t)=\mu S(t) \mathrm{d} t+S(t) \mathrm{d} Y(t), \quad t \geq 0
$$

Applying Itô's formula shows as in the standard Black-Scholes model that the asset price $S$ can be represented by

$$
S(t)=S(0) \exp \left(Y(t)+\left(\mu-\frac{1}{2} \sigma^{2}\right) t\right), \quad t \geq 0
$$

In the case, when the feedback traders are absent, i.e., $\beta_{j}=0$, for all $j=1, \ldots, N$, we have $\mathrm{d} Y(t)=\sigma \mathrm{d} W(t)$, in which case $S$ is a Geometric Brownian motion, evolving according to

$$
\mathrm{d} S(t)=\mu S(t) \mathrm{d} t+\sigma S(t) \mathrm{d} W(t), \quad t \geq 0 .
$$

In this case this model coincides with the standard Black-Scholes model and can be considered as a generalisation of it.

Needless to say there are numerous results in the literature dealing with modeling financial problems via stochastic delay differential equation (see, e.g. Arriojas et al. [3]), but the above presented example was one of the most motivational one for the birth of this thesis.

\section{$1.2 \quad$ Local asymptotic normality}

One of the most important and fundamental notion in asymptotic statistics is the local asymptotic normality. As one can read in the book of van der Vaart [46], a sequence of statistical models is locally asymptotic normal if, asymptotically, their likelihood ratio processes are similar to those for a normal location parameter. Local asymptotic normality implies convergence of the models to a Gaussian model after a rescaling of the parameter. The precise definition of local asymptotic normality and the other notions and results of asymptotic statistics can be found in Chapter 2. In this section some parts of the book of van der Vaart [46, Chapter 7] be recalled to introduce the basic concept of asymptotic statistics.

Suppose we observe a sample $X_{1}, \ldots, X_{n}$ from a distribution $\mathbb{P}_{\theta}$ on some measurable space $(\mathcal{X}, \mathcal{A})$ indexed by a parameter $\theta$ that ranges over on open subset $\Theta$ of $\mathbb{R}^{k}$. For simplicity, assume that $k$ equals to one. Then the full observation is a single observation from the product $\mathbb{P}_{\theta}^{n}$ of $n$ copies of $\mathbb{P}_{\theta}$, and the statistical model is completely described as the collection of probability measures $\left\{\mathbb{P}_{\theta}^{n}: \theta \in \Theta\right\}$ on the sample space $\left(\mathcal{X}^{n}, \mathcal{A}^{n}\right)$. In this context we shall speak of a statistical experiment, rather than of a statistical model. It is shown that many statistical experiments can be approximated by Gaussian experiments after a suitable reparametrization.

The reparametrization is centered around a fixed parameter $\theta_{0}$, which should be regarded as known. We define a local parameter $h=\sqrt{n}\left(\theta-\theta_{0}\right)$, rewrite $\mathbb{P}_{\theta}^{n}$ as $\mathbb{P}_{\theta_{0}+h / \sqrt{n}}^{n}$, and thus 
obtain an experiment with parameter $h$. In the case of local asymptotic normality, for large $n$, the experiments

$$
\left(\mathbb{P}_{\theta_{0}+\frac{h}{\sqrt{n}}}^{n}: h \in \mathbb{R}\right) \quad \text { and } \quad\left(\mathcal{N}\left(h, J_{\theta_{0}}^{-1}\right): h \in \mathbb{R}\right)
$$

are similar in statistical properties, where the second experiment consists of observing a single observation from a normal distribution with mean $h$ and known covariance matrix (equal to the inverse of the Fisher information matrix). This is a simple experiment, which is easy to analyze, whence the approximation yields much information about the asymptotic properties of the original experiments. This information concerns both asymptotic optimality theory and the behaviour of statistical procedures such as maximum likelihood estimator and hypothesis testing, see Section 2.2 .

To see the similarity of the experiments in (1.2.1), we show the similarity of the corresponding likelihood ratio processes. For this, assume for simplicity, that exists the density $p_{\theta}$ of $\mathbb{P}_{\theta}$ with respect to some measure $\mu$, and that the $\log$-likelihood $\ell_{\theta}(x)=\log p_{\theta}(x)$ is twice-differentiable with respect to $\theta$, for every $x$, with derivaties $\dot{\ell}_{\theta}(x)$ and $\ddot{\ell}_{\theta}(x)$. Then, for every fixed $x$,

$$
\log \frac{p_{\theta+h}}{p_{\theta}}(x)=h \dot{\ell}_{\theta}(x)+\frac{1}{2} h^{2} \ddot{\ell}_{\theta}(x)+\mathrm{o}_{x}\left(h^{2}\right) .
$$

The subscript $x$ in the reminder term is a reminder of the fact that this term depend on $x$ as well as on $h$. It follows that

$$
\log \prod_{i=1}^{n} \frac{p_{\theta+\frac{h}{\sqrt{n}}}}{p_{\theta}}\left(X_{i}\right)=\frac{h}{\sqrt{n}} \sum_{i=1}^{n} \dot{\ell}_{\theta}\left(X_{i}\right)+\frac{1}{2} \frac{h^{2}}{n} \sum_{i=1}^{n} \ddot{\ell}_{\theta}\left(X_{i}\right)+\operatorname{Rem}_{n} .
$$

Here the score has mean zero, $\int \dot{\ell}_{\theta} \mathrm{d} \mathbb{P}_{\theta}=0$, and $-\int \ddot{\ell}_{\theta} \mathrm{d} \mathbb{P}_{\theta}=\int\left(\dot{\ell}_{\theta}\right)^{2} \mathrm{~d} \mathbb{P}_{\theta}=J_{\theta}$ equals the Fisher information for $\theta$ (see, van der Vaart [46, Section 5.5]). Hence the first term can be rewritten as $h \Delta_{n, \theta}$, where $\Delta_{n, \theta}=n^{-1 / 2} \sum_{i=1}^{n} \dot{\ell}_{\theta}\left(X_{i}\right)$ is asymptotically normal with zero mean and variance $J_{\theta}$, by the central limit theorem. Furthermore, the second term in the expansion is asymptotically equivalent to $-\frac{1}{2} h^{2} J_{\theta}$, by the law of large numbers. The remainder term should behave as $\mathrm{o}(1 / n)$ times a sum of $n$ terms and hopefully is asymptotically negligible. Consequently, under suitable conditions we have, for every $h$,

$$
\log \prod_{i=1}^{n} \frac{p_{\theta+\frac{h}{\sqrt{n}}}}{p_{\theta}}\left(X_{i}\right)=h \Delta_{n, \theta}-\frac{1}{2} h^{2} J_{\theta}+\mathrm{o}_{\mathbb{P}_{\theta}}(1) .
$$

Because this expansion concerns the likelihood process in a neighborhood of $\theta$, we speak of local asymptotic normality of the sequence of models $\left\{\mathbb{P}_{\theta}^{n}: \theta \in \Theta\right\}$.

The second experiment in (1.2.1), thus the experiment, which consists of observing a single observation $X$ with the distribution $\mathcal{N}\left(h, J_{\theta}^{-1}\right)$. The log-likelihood ratio process of this experiment equals

$$
\log \frac{\mathrm{d} \mathcal{N}\left(h, J_{\theta}^{-1}\right)}{\mathrm{d} \mathcal{N}\left(0, J_{\theta}^{-1}\right)}(X)=h J_{\theta} X-\frac{1}{2} h^{2} J_{\theta} .
$$

If we compare (1.2.2) and (1.2.3), we can see, that the corresponding likelihood ratio processes of the experiments (1.2.1) are asymptotically equal in distribution. 


\subsection{Historical background}

The list of the papers dealing with investigation of the local asymptotic properties of delay models is not so extensive. The majority of the literature is the works by Gushchin, Küchler and their co-authors. One of the first paper was made in 1999 by Gushchin and Küchler [16]. They study the local asymptotic properties of the equation

$$
\mathrm{d} X(t)=(a X(t)+b X(t-1)) \mathrm{d} t+\mathrm{d} W(t) .
$$

It is shown, that eleven different cases - consisting LAN, LAMN, PLAMN and LAQ (for the definitions, see, Section 2.1) - are possible if the value of the parameter $\boldsymbol{\theta}=(a, b)$ runs through $\mathbb{R}^{2}$. In 2001 an addendum was published by Gushchin and Küchler [18]. In this paper they strengthen the result in one of the cases. With an other, periodic normalization function they show that in on of the PLAMN case the usual LAMN property holds.

In 2003 the paper written by Gushchin and Küchler [19] consists the equation

$$
\mathrm{d} X(t)=\int_{[-r, 0]} X(t+u) a_{\boldsymbol{\theta}}(\mathrm{d} u) \mathrm{d} t+\mathrm{d} W(t),
$$

which is a more complex model then the previous one. Here $a_{\boldsymbol{\theta}}$ is a finite signed measure depending on the parameter $\boldsymbol{\theta}$, which belongs to an open set $\Theta \subseteq \mathbb{R}^{k}$. Conditions are derived under which this model satisfies the LAN property. These conditions are the stationarity of the solution and some regularity conditions for the smoothness of the dependence of $a_{\boldsymbol{\theta}}$ on $\boldsymbol{\theta}$. A necessary and sufficient condition is proved for stationary solutions in the paper by Gushchin and Küchler [17].

The exhaustive doctoral dissertation written in 2001 by Putschke [37] study the model

$$
\mathrm{d} X(t)=\sum_{i=0}^{N} \theta_{i} X\left(t-r_{i}\right) \mathrm{d} t+\mathrm{d} W(t),
$$

where $r_{i} \in \mathbb{R}_{+}$and $0=r_{0}<r_{1}<\cdots<r_{N}$. Depending on the parameter $\boldsymbol{\theta}=\left(\theta_{0}, \ldots, \theta_{N}\right) \in$ $\mathbb{R}^{N+1}$ the local asymptotic properties are derived.

The investigation of the asymptotic behaviour of the maximum likelihood estimator is strongly connected to the local asymptotic properties. Küchler and Kutoyants [27] investigated in 2000 the equation

$$
\mathrm{d} X(t)=b X(t-\theta) \mathrm{d} t+\mathrm{d} W(t),
$$

where $b$ is a known negative parameter and the delay parameter $\theta \in \mathbb{R}$ is unknown. It is shown that the MLE is consistent and its limit distribution is described. Gushchin and Küchler [20] considered a generalisation of this model in 2011. They study the model

$$
\mathrm{d} X(t)=\int_{[-r, 0]} X(t+u) a_{\theta}(\mathrm{d} u) \mathrm{d} t+\mathrm{d} W(t),
$$


with stationary solutions, where the parameter $\boldsymbol{\theta} \in \Theta, \Theta=\left(\theta_{0}, \theta_{1}\right),-\infty<\theta_{0}<0<\theta_{1}<\infty$ and the finite signed measures $a_{\boldsymbol{\theta}}$ are of the form $a_{\boldsymbol{\theta}}=a+b_{\boldsymbol{\theta}}-b$, where $a$ and $b$ are finite signed measures, and $b_{\boldsymbol{\theta}}$ is the translate of $b$ by $\boldsymbol{\theta}$, namely $b_{\boldsymbol{\theta}}(B)=b(B-\boldsymbol{\theta})$ for any Borel set $B$. The limit behaviour of the normalized likelihoods are studied, and it turns out, that under some additional conditions the limit can be described with the help of a fractional Brownian motion with index $H \in[1 / 2,1]$, and every $H \in[1 / 2,1]$ may occur in this framework.

All of the mentioned studies and this thesis were concerned with continuous observation of the solution process. Küchler and Sørensen [28], [29] considered delayed models with discrete time observations.

Nonparametric estimators for delayed stochastic differential equations were investigated by Reiß [38], [39], [40]. 



\section{Chapter 2}

\section{Asymptotic statistics}

In this chapter the main definitions and results from the topic of asymptotic statistics are recalled. Furthermore these results are illustrated on the Heston model, which is a well-known model in financial mathematics. At the beginning in Section 2.1 the local asymptotic properties of the likelihood function or likelihood ratios of statistical experiments are defined and the Le Cam's lemmas are recalled. In Section 2.2 some useful consequences are stated. Finally in Section 2.3 the Heston model is considered. The results have been published in Benke and Pap [12].

\subsection{Local asymptotic properties of statistical experiments}

The following definitions and statements concerning quadratic approximations to likelihood ratios based on Jeganathan [26], Le Cam and Yang [31], van der Vaart [46], Shiryaev and Spokoiny [44], Kutoyants [30] and Höpfner [23].

If $\mathbb{P}$ and $\mathbb{Q}$ are probability measures on a measurable space $(X, \mathcal{X})$, then

$$
\frac{\mathrm{d} \mathbb{P}}{\mathrm{d} \mathbb{Q}}: X \rightarrow \mathbb{R}_{+}
$$

denotes the Radon-Nykodym derivative of the absolutely continuous part of $\mathbb{P}$ with respect to $\mathbb{Q}$. If $(X, \mathcal{X}, \mathbb{P})$ is a probability space and $(Y, \mathcal{Y})$ is a measurable space, then the distribution of a measurable mapping $\xi: X \rightarrow Y$ under $\mathbb{P}$ will be denoted by $\mathcal{L}(\xi \mid \mathbb{P})$ (i.e., $\mathcal{L}(\xi \mid \mathbb{P})$ is the probability measure on $(Y, \mathcal{Y})$ defined by $\mathcal{L}(\xi \mid \mathbb{P})(B):=\mathbb{P}(\xi \in B), B \in \mathcal{Y})$.

2.1.1 Definition. A statistical experiment is a triplet $\left(X, \mathcal{X},\left\{\mathbb{P}_{\theta}: \theta \in \Theta\right\}\right)$, where $(X, \mathcal{X})$ is a measurable space and $\left\{\mathbb{P}_{\theta}: \theta \in \Theta\right\}$ is a family of probability measures on $(X, \mathcal{X})$. Its likelihood ratio process with base $\theta_{0} \in \Theta$ is the stochastic process

$$
\left(\frac{\mathrm{d} \mathbb{P}_{\theta}}{d \mathbb{P}_{\theta_{0}}}\right)_{\theta \in \Theta} .
$$


2.1.2 Definition. A family $\left(X_{T}, \mathcal{X}_{T},\left\{\mathbb{P}_{\theta, T}: \theta \in \Theta\right\}\right)_{T \in \mathbb{R}_{++}}$of statistical experiments converges to a statistical experiment $\left(X, \mathcal{X},\left\{\mathbb{P}_{\theta}: \theta \in \Theta\right\}\right)$ as $T \rightarrow \infty$ if, for every finite subset $H \subset \Theta$ and every $\theta_{0} \in \Theta$,

$$
\mathcal{L}\left(\left(\frac{\mathrm{d} \mathbb{P}_{\theta, T}}{\mathrm{~d} \mathbb{P}_{\theta_{0}, T}}\right)_{\theta \in H} \mid \mathbb{P}_{\theta_{0}, T}\right) \Rightarrow \mathcal{L}\left(\left(\frac{\mathrm{d} \mathbb{P}_{\theta}}{\mathrm{d} \mathbb{P}_{\theta_{0}}}\right)_{\theta \in H} \mid \mathbb{P}_{\theta_{0}}\right) \quad \text { as } T \rightarrow \infty,
$$

i.e., the finite dimensional distributions of the likelihood ratio process $\left(\frac{\mathrm{d} \mathbb{P}_{\theta, T}}{\mathrm{~d} \mathbb{P}_{\theta_{0}, T}}\right)_{\theta \in \Theta}$ under $\mathbb{P}_{\theta_{0}, T}$ converges to the finite dimensional distributions of the likelihood ratio process $\left(\frac{d \mathbb{P}_{\theta}}{d \mathbb{P}_{\theta_{0}}}\right)_{\theta \in \Theta}$ under $\mathbb{P}_{\theta_{0}}$ as $T \rightarrow \infty$.

If $\left(X_{T}, \mathcal{X}_{T}, \mathbb{P}_{T}\right), T \in \mathbb{R}_{++}$, are probability spaces and $f_{T}: X_{T} \rightarrow \mathbb{R}^{p}, T \in \mathbb{R}_{++}$, are measurable functions, then

$$
f_{T} \stackrel{\mathbb{P}_{T}}{\longrightarrow} 0 \quad \text { or } \quad f_{T}=\mathrm{O}_{\mathbb{P}_{T}}(1) \quad \text { as } T \rightarrow \infty
$$

denotes convergence in $\left(\mathbb{P}_{T}\right)_{T \in \mathbb{R}_{++}}$-probabilities to 0 as $T \rightarrow \infty$, i.e., $\mathbb{P}_{T}\left(\left\|f_{T}\right\|>\varepsilon\right) \rightarrow 0$ as $T \rightarrow \infty$ for all $\varepsilon \in \mathbb{R}_{++}$. Moreover,

$$
f_{T}=\mathrm{O}_{\mathbb{P}_{T}}(1), \quad T \in \mathbb{R}_{++},
$$

denotes boundedness in $\left(\mathbb{P}_{T}\right)_{T \in \mathbb{R}_{++}}$-probabilities, i.e., $\sup _{T \in \mathbb{R}_{++}} \mathbb{P}_{T}\left(\left\|f_{T}\right\|>K\right) \rightarrow 0$ as $K \rightarrow \infty$.

2.1.3 Remark. Note that if $(\Omega, \mathcal{A}, \mathbb{P})$ is a probability space and for each $T \in \mathbb{R}_{++}, \xi_{T}$ : $\Omega \rightarrow X_{T}$ is a random element with $\mathcal{L}\left(\xi_{T} \mid \mathbb{P}\right)=\mathbb{P}_{T}$, then $f_{T}=\mathrm{o}_{\mathbb{P}_{T}}(1)$ as $T \rightarrow \infty$ or $f_{T}=\mathrm{O}_{\mathbb{P}_{T}}(1), \quad T \in \mathbb{R}_{++}$, if and only if $f_{T} \circ \xi_{T}=\mathrm{o}_{\mathbb{P}}(1)$ as $T \rightarrow \infty$ or $f_{T} \circ \xi_{T}=\mathrm{O}_{\mathbb{P}}(1)$, $T \in \mathbb{R}_{++}$, respectively. Indeed, $\mathbb{P}_{T}\left(\left\|f_{T}\right\|>c\right)=\mathbb{P}\left(\left\|f_{T}\left(\xi_{T}\right)\right\|>c\right)$ for all $T \in \mathbb{R}_{++}$and all $c \in \mathbb{R}_{++}$. Moreover, $f_{T}=\mathrm{O}_{\mathbb{P}_{T}}(1), T \in \mathbb{R}_{++}$, if and only if the family $\left(\mathcal{L}\left(f_{T} \mid \mathbb{P}_{T}\right)\right)_{T \in \mathbb{R}_{++}}$ of probability measures is tight, and hence, for each sequence $T_{n} \in \mathbb{R}_{++}, n \in \mathbb{N}$, with $T_{n} \rightarrow \infty$ as $n \rightarrow \infty$, there exist a subsequence $T_{n_{k}}, k \in \mathbb{N}$, and a probability measure $\mu$ on $\left(\mathbb{R}^{p}, \mathcal{B}\left(\mathbb{R}^{p}\right)\right)$, such that $\mathcal{L}\left(f_{T_{n_{k}}} \mid \mathbb{P}_{T_{n_{k}}}\right) \Rightarrow \mu$ as $k \rightarrow \infty$. In this case, $\mu$ is called an accumulation point of the family $\left(\mathcal{L}\left(f_{T} \mid \mathbb{P}_{T}\right)\right)_{T \in \mathbb{R}_{++}}$.

2.1.4 Definition. Let $\Theta \subset \mathbb{R}^{p}$ be an open set. A family $\left(X_{T}, \mathcal{X}_{T},\left\{\mathbb{P}_{\boldsymbol{\theta}, T}: \boldsymbol{\theta} \in \Theta\right\}\right)_{T \in \mathbb{R}_{++}}$of statistical experiments is said to have locally asymptotically quadratic (LAQ) likelihood ratios at $\boldsymbol{\theta} \in \Theta$ if there exist (scaling) matrices $\boldsymbol{r}_{\boldsymbol{\theta}, T} \in \mathbb{R}^{p \times p}, T \in \mathbb{R}_{++}$, measurable functions (statistics) $\boldsymbol{\Delta}_{\boldsymbol{\theta}, T}: X_{T} \rightarrow \mathbb{R}^{p}, T \in \mathbb{R}_{++}$, and $\boldsymbol{J}_{\boldsymbol{\theta}, T}: X_{T} \rightarrow \mathbb{R}^{p \times p}, T \in \mathbb{R}_{++}$, such that

$$
\log \frac{\mathrm{d} \mathbb{P}_{\boldsymbol{\theta}+\boldsymbol{r}_{\boldsymbol{\theta}, T} \boldsymbol{h}_{T}, T}}{\mathrm{~d} \mathbb{P}_{\boldsymbol{\theta}, T}}=\boldsymbol{h}_{T}^{\top} \boldsymbol{\Delta}_{\boldsymbol{\theta}, T}-\frac{1}{2} \boldsymbol{h}_{T}^{\top} \boldsymbol{J}_{\boldsymbol{\theta}, T} \boldsymbol{h}_{T}+\mathrm{O}_{\mathbb{P}_{\boldsymbol{\theta}, T}} \text { (1) } \quad \text { as } T \rightarrow \infty
$$

whenever $\boldsymbol{h}_{T} \in \mathbb{R}^{p}, \quad T \in \mathbb{R}_{++}$, is a bounded family satisfying $\boldsymbol{\theta}+\boldsymbol{r}_{\boldsymbol{\theta}, T} \boldsymbol{h}_{T} \in \Theta$ for all $T \in \mathbb{R}_{++}$,

$$
\left(\boldsymbol{\Delta}_{\boldsymbol{\theta}, T}, \boldsymbol{J}_{\boldsymbol{\theta}, T}\right)=\mathrm{O}_{\mathbb{P}_{\boldsymbol{\theta}, T}}(1), \quad T \in \mathbb{R}_{++},
$$


and for each accumulation point $\mu_{\boldsymbol{\theta}}$ of the family $\left(\mathcal{L}\left(\left(\boldsymbol{\Delta}_{\boldsymbol{\theta}, T}, \boldsymbol{J}_{\boldsymbol{\theta}, T}\right) \mid \mathbb{P}_{\boldsymbol{\theta}, T}\right)\right)_{T \in \mathbb{R}_{++}}$as $T \rightarrow \infty$, which is a probability measure on $\left(\mathbb{R}^{p} \times \mathbb{R}^{p \times p}, \mathcal{B}\left(\mathbb{R}^{p} \times \mathbb{R}^{p \times p}\right)\right)$, we have

$$
\mu_{\boldsymbol{\theta}}\left(\left\{(\boldsymbol{\Delta}, \boldsymbol{J}) \in \mathbb{R}^{p} \times \mathbb{R}^{p \times p}: \boldsymbol{J} \text { is symmetric and strictly positive definite }\right\}\right)=1
$$

and

$$
\int_{\mathbb{R}^{p} \times \mathbb{R}^{p \times p}} \exp \left\{\boldsymbol{h}^{\top} \boldsymbol{\Delta}-\frac{1}{2} \boldsymbol{h}^{\top} \boldsymbol{J} \boldsymbol{h}\right\} \mu_{\boldsymbol{\theta}}(\mathrm{d} \boldsymbol{\Delta}, \mathrm{d} \boldsymbol{J})=1
$$

whenever $\boldsymbol{h} \in \mathbb{R}^{p}$ such that there exist $T_{k} \in \mathbb{R}_{++}, k \in \mathbb{N}$, and $\boldsymbol{h}_{T_{k}} \in \mathbb{R}^{p}, k \in \mathbb{N}$, with $\boldsymbol{h}_{T_{k}} \rightarrow \boldsymbol{h}$ as $k \rightarrow \infty, \boldsymbol{\theta}+\boldsymbol{r}_{\boldsymbol{\theta}, T_{k}} \boldsymbol{h}_{T_{k}} \in \Theta$ for all $k \in \mathbb{N}$.

2.1.5 Definition. Let $\Theta \subset \mathbb{R}^{p}$ be an open set. A family $\left(X_{T}, \mathcal{X}_{T},\left\{\mathbb{P}_{\boldsymbol{\theta}, T}: \boldsymbol{\theta} \in \Theta\right\}\right)_{T \in \mathbb{R}_{++}}$of statistical experiments is said to have locally asymptotically mixed normal (LAMN) likelihood ratios at $\boldsymbol{\theta} \in \Theta$ if it is $L A Q$ at $\boldsymbol{\theta} \in \Theta$, and for each accumulation point $\mu_{\boldsymbol{\theta}}$ of the family $\left(\mathcal{L}\left(\left(\boldsymbol{\Delta}_{\boldsymbol{\theta}, T}, \boldsymbol{J}_{\boldsymbol{\theta}, T}\right) \mid \mathbb{P}_{\boldsymbol{\theta}, T}\right)\right)_{T \in \mathbb{R}_{++}}$as $T \rightarrow \infty$, we have

$$
\int_{\mathbb{R}^{p} \times B} \mathrm{e}^{\mathrm{i} \boldsymbol{h}^{\top} \boldsymbol{\Delta}} \mu_{\boldsymbol{\theta}}(\mathrm{d} \boldsymbol{\Delta}, \mathrm{d} \boldsymbol{J})=\int_{\mathbb{R}^{p} \times B} \mathrm{e}^{-\boldsymbol{h}^{\top} \boldsymbol{J} h / 2} \mu_{\boldsymbol{\theta}}(\mathrm{d} \boldsymbol{\Delta}, \mathrm{d} \boldsymbol{J}), \quad B \in \mathcal{B}\left(\mathbb{R}^{p \times p}\right), \quad \boldsymbol{h} \in \mathbb{R}^{p},
$$

i.e., the conditional distribution of $\boldsymbol{\Delta}$ given $\boldsymbol{J}$ under $\mu_{\boldsymbol{\theta}}$ is $\mathcal{N}_{p}(0, \boldsymbol{J})$, or, equivalently, $\mu_{\boldsymbol{\theta}}=\mathcal{L}\left(\left(\eta_{\boldsymbol{\theta}} \mathcal{Z}, \eta_{\boldsymbol{\theta}} \eta_{\boldsymbol{\theta}}^{\top}\right) \mid \mathbb{P}\right)$, where $\mathcal{Z}: \Omega \rightarrow \mathbb{R}^{p}$ and $\eta_{\boldsymbol{\theta}}: \Omega \rightarrow \mathbb{R}^{p \times p}$ are independent random elements on a probability space $(\Omega, \mathcal{F}, \mathbb{P})$ such that $\mathcal{L}(\mathcal{Z} \mid \mathbb{P})=\mathcal{N}_{p}\left(0, \boldsymbol{I}_{p}\right)$.

2.1.6 Definition. Let $\Theta \subset \mathbb{R}^{p}$ be an open set. A family $\left(X_{T}, \mathcal{X}_{T},\left\{\mathbb{P}_{\boldsymbol{\theta}, T}: \boldsymbol{\theta} \in \Theta\right\}\right)_{T \in \mathbb{R}_{++}}$ of statistical experiments is said to have locally asymptotically normal ( $L A N$ ) likelihood ratios at $\boldsymbol{\theta} \in \Theta$ if it is $L A M N$ at $\boldsymbol{\theta} \in \Theta$, and for each accumulation point $\mu_{\boldsymbol{\theta}}$ of the family $\left(\mathcal{L}\left(\left(\boldsymbol{\Delta}_{\boldsymbol{\theta}, T}, \boldsymbol{J}_{\boldsymbol{\theta}, T}\right) \mid \mathbb{P}_{\boldsymbol{\theta}, T}\right)\right)_{T \in \mathbb{R}_{++}}$as $T \rightarrow \infty$, we have

$$
\mu_{\boldsymbol{\theta}}=\mathcal{N}_{p}\left(0, \boldsymbol{J}_{\boldsymbol{\theta}}\right) \times \delta_{\boldsymbol{J}_{\theta}}
$$

with some symmetric, strictly positive definite matrix $\boldsymbol{J}_{\boldsymbol{\theta}} \in \mathbb{R}^{p \times p}$, where $\delta_{\boldsymbol{J}_{\boldsymbol{\theta}}}$ denotes the Dirac measure on $\left(\mathbb{R}^{p \times p}, \mathcal{B}\left(\mathbb{R}^{p \times p}\right)\right)$, concentrated in $\boldsymbol{J}_{\boldsymbol{\theta}}$. The matrix $\boldsymbol{J}_{\boldsymbol{\theta}}$ is called the information matrix.

These definitions of LAQ, LAMN and LAN are well-known. However in most of the applications, including in the most of the models in this thesis, a special case occurs, namely when the family $\left(\mathcal{L}\left(\left(\boldsymbol{\Delta}_{\boldsymbol{\theta}, T}, \boldsymbol{J}_{\boldsymbol{\theta}, T}\right) \mid \mathbb{P}_{\boldsymbol{\theta}, T}\right)\right)_{T \in \mathbb{R}_{++}}$has only one accumulation point as $T \rightarrow \infty$, hence a pair of random variable $\left(\boldsymbol{\Delta}_{\boldsymbol{\theta}}, \boldsymbol{J}_{\boldsymbol{\theta}}\right) \in\left(\mathbb{R}^{p}, \mathbb{R}^{p \times p}\right)$ exists such that

$$
\left(\boldsymbol{\Delta}_{\boldsymbol{\theta}, T}, \boldsymbol{J}_{\boldsymbol{\theta}, T}\right) \stackrel{\mathcal{D}}{\longrightarrow}\left(\boldsymbol{\Delta}_{\boldsymbol{\theta}}, \boldsymbol{J}_{\boldsymbol{\theta}}\right) \quad \text { as } T \rightarrow \infty
$$

Nevertheless there are examples, when not only one accumulation point exists. This is the case in the following definiton, which was introduced by Gushchin and Küchler [16]. 
2.1.7 Definition. Let $\Theta \subset \mathbb{R}^{p}$ be an open set. A family $\left(X_{T}, \mathcal{X}_{T},\left\{\mathbb{P}_{\boldsymbol{\theta}, T}: \boldsymbol{\theta} \in \Theta\right\}\right)_{T \in \mathbb{R}_{++}}$of statistical experiments is said to have periodic locally asymptotically mixed normal (PLAMN) likelihood ratios at $\boldsymbol{\theta} \in \Theta$ if it is $L A Q$ at $\boldsymbol{\theta} \in \Theta$, and

$$
\left(\boldsymbol{\Delta}_{\boldsymbol{\theta}, k D+d}, \boldsymbol{J}_{\boldsymbol{\theta}, k D+d}\right) \stackrel{\mathcal{D}}{\longrightarrow}\left(\boldsymbol{\Delta}_{\boldsymbol{\theta}}(d), \boldsymbol{J}_{\boldsymbol{\theta}}(d)\right) \quad \text { as } k \rightarrow \infty
$$

for all $d \in[0, D)$, and for each $d \in[0, D)$, the conditional distribution of $\boldsymbol{\Delta}_{\theta}(d)$ given $\boldsymbol{J}_{\boldsymbol{\theta}}(d)$ is $\mathcal{N}_{p}\left(0, \boldsymbol{J}_{\boldsymbol{\theta}}(d)\right)$, or, equivalently, there exist a random vector $\mathcal{Z}: \Omega \rightarrow \mathbb{R}^{p}$ and a random matrix $\eta_{\boldsymbol{\theta}}(d): \Omega \rightarrow \mathbb{R}^{p \times p}$ such that they are independent, $\mathcal{Z} \stackrel{\mathcal{D}}{=} \mathcal{N}_{p}\left(0, \boldsymbol{I}_{p}\right)$, and $\boldsymbol{\Delta}_{\boldsymbol{\theta}}(d)=\eta_{\boldsymbol{\theta}}(d) \mathcal{Z}, \quad \boldsymbol{J}_{\boldsymbol{\theta}}(d)=\eta_{\boldsymbol{\theta}}(d) \eta_{\boldsymbol{\theta}}^{\top}(d)$.

According to the definition of LAMN (2.1.5) PLAMN is a special case of LAMN, when the family $\left(\mathcal{L}\left(\left(\boldsymbol{\Delta}_{\boldsymbol{\theta}, T}, \boldsymbol{J}_{\boldsymbol{\theta}, T}\right) \mid \mathbb{P}_{\boldsymbol{\theta}, T}\right)\right)_{T \in \mathbb{R}_{++}}$has uncountably infinitely many accumulation points as $T \rightarrow \infty$, and these accumulation points can be reached by arithmetic subsequences with the same common difference $(D)$, which is called period, but with different initial terms $(d)$.

We will need Le Cam's first lemma, see, e.g, Lemma 6.4 in van der Vaart [46] or Höpfner [23, Lemma 3.5]. For this the definition of contiguity of families of probability measures is recalled.

2.1.8 Definition. Let $\left(X_{T}, \mathcal{X}_{T}\right), T \in \mathbb{R}_{++}$, be measurable spaces. For each $T \in \mathbb{R}_{++}$, let $\mathbb{P}_{T}$ and $\mathbb{Q}_{T}$ be probability measures on $\left(X_{T}, \mathcal{X}_{T}\right)$. The family $\left(\mathbb{Q}_{T}\right)_{T \in \mathbb{R}_{++}}$is said to be contiguous with respect to the family $\left(\mathbb{P}_{T}\right)_{T \in \mathbb{R}_{++}}$if $\mathbb{Q}_{T}\left(A_{T}\right) \rightarrow 0$ as $T \rightarrow \infty$ whenever $A_{T} \in \mathcal{X}_{T}, T \in \mathbb{R}_{++}$, such that $\mathbb{P}_{T}\left(A_{T}\right) \rightarrow 0$ as $T \rightarrow \infty$. This will be denoted by $\left(\mathbb{Q}_{T}\right)_{T \in \mathbb{R}_{++}} \triangleleft\left(\mathbb{P}_{T}\right)_{T \in \mathbb{R}_{++}}$. The families $\left(\mathbb{P}_{T}\right)_{T \in \mathbb{R}_{++}}$and $\left(\mathbb{Q}_{T}\right)_{T \in \mathbb{R}_{++}}$are said to be mutually contiguous if both $\left(\mathbb{P}_{T}\right)_{T \in \mathbb{R}_{++}} \triangleleft\left(\mathbb{Q}_{T}\right)_{T \in \mathbb{R}_{++}}$and $\left(\mathbb{Q}_{T}\right)_{T \in \mathbb{R}_{++}} \triangleleft\left(\mathbb{P}_{T}\right)_{T \in \mathbb{R}_{++}}$hold.

2.1.9 Lemma. (Le Cam's first lemma) Let $\left(X_{T}, \mathcal{X}_{T}\right), T \in \mathbb{R}_{++}$, be measurable spaces. For each $T \in \mathbb{R}_{++}$, let $\mathbb{P}_{T}$ and $\mathbb{Q}_{T}$ be probability measures on $\left(X_{T}, \mathcal{X}_{T}\right)$. Then the following statements are equivalent:

(i) $\left(\mathbb{Q}_{T}\right)_{T \in \mathbb{R}_{++}} \triangleleft\left(\mathbb{P}_{T}\right)_{T \in \mathbb{R}_{++}}$;

(ii) If $\mathcal{L}\left(\frac{\mathrm{d} \mathbb{P}_{T_{k}}}{\mathrm{~d} \mathbb{Q}_{T_{k}}} \mid \mathbb{Q}_{T_{k}}\right) \Rightarrow \nu$ as $k \rightarrow \infty$ for some sequence $\left(T_{k}\right)_{k \in \mathbb{N}}$ with $T_{k} \rightarrow \infty$ as $T \rightarrow \infty$, where $\nu$ is a probability measure on $\left(\mathbb{R}_{+}, \mathcal{B}\left(\mathbb{R}_{+}\right)\right)$, then $\nu\left(\mathbb{R}_{++}\right)=1$;

(iii) If $\mathcal{L}\left(\frac{\mathrm{d}_{T_{k}}}{\mathrm{~d} \mathbb{P}_{T_{k}}} \mid \mathbb{P}_{T_{k}}\right) \Rightarrow \mu$ as $k \rightarrow \infty$ for some sequence $\left(T_{k}\right)_{k \in \mathbb{N}}$ with $T_{k} \rightarrow \infty$ as $T \rightarrow \infty$, where $\mu$ is a probability measure on $\left(\mathbb{R}_{+}, \mathcal{B}\left(\mathbb{R}_{+}\right)\right)$, then $\int_{\mathbb{R}_{++}} x \mu(\mathrm{d} x)=1$;

(iv) $\mathcal{L}\left(f_{T} \mid \mathbb{Q}_{T}\right) \Rightarrow 0$ as $T \rightarrow \infty$ whenever $f_{T}: X_{T} \rightarrow \mathbb{R}^{p}, \quad T \in \mathbb{R}_{++}$, are measurable functions and $\mathcal{L}\left(f_{T} \mid \mathbb{P}_{T}\right) \Rightarrow 0$ as $T \rightarrow \infty$.

We will need a version of general form of Le Cam's third lemma, see, e.g., van der Vaart [46, Theorem 6.6] or Höpfner [23, Lemma 3.6]. 
2.1.10 Theorem. Let $\left(X_{T}, \mathcal{X}_{T}\right), T \in \mathbb{R}_{++}$, be measurable spaces. For each $T \in \mathbb{R}_{++}$, let $\mathbb{P}_{T}$ and $\mathbb{Q}_{T}$ be probability measures on $\left(X_{T}, \mathcal{X}_{T}\right)$. Let $f_{T}: X_{T} \rightarrow \mathbb{R}^{p}, T \in \mathbb{R}_{++}$, be measurable functions. Suppose that the family $\left(\mathbb{Q}_{T}\right)_{T \in \mathbb{R}_{++}}$is contiguous with respect to the family $\left(\mathbb{P}_{T}\right)_{T \in \mathbb{R}_{++}}$and

$$
\mathcal{L}\left(\left(f_{T}, \frac{\mathrm{d} \mathbb{Q}_{T}}{\mathrm{~d} \mathbb{P}_{T}}\right) \mid \mathbb{P}_{T}\right) \Rightarrow \nu \quad \text { as } \quad T \rightarrow \infty,
$$

where $\nu$ is a probability measure on $\left(\mathbb{R}^{p} \times \mathbb{R}_{+}, \mathcal{B}\left(\mathbb{R}^{p} \times \mathbb{R}_{+}\right)\right)$. Then $\mathcal{L}\left(f_{T} \mid \mathbb{Q}_{T}\right) \Rightarrow \mu$ as $T \rightarrow \infty$, where $\mu$ is the probability measure on $\left(\mathbb{R}^{p}, \mathcal{B}\left(\mathbb{R}^{p}\right)\right)$ given by

$$
\mu(B):=\int_{\mathbb{R}^{p} \times \mathbb{R}_{+}} \mathbb{1}_{B}(f) V \nu(\mathrm{d} f, \mathrm{~d} V), \quad B \in \mathcal{B}\left(\mathbb{R}^{p}\right) .
$$

The following convergence theorem is Proposition 1 in Jeghanathan [26]. In fact, it is a generalization of Theorems 9.4 and 9.8 of van der Vaart [46], which are valid for LAMN and LAN families of experiments. For completeness, here is the proof as well.

2.1.11 Theorem. Let $\Theta \subset \mathbb{R}^{p}$ be an open set. Let $\left(X_{T}, \mathcal{X}_{T},\left\{\mathbb{P}_{\boldsymbol{\theta}, T}: \boldsymbol{\theta} \in \Theta\right\}\right)_{T \in \mathbb{R}_{++}}$be a family of statistical experiments. Assume that $L A Q$ is satisfied at $\boldsymbol{\theta} \in \Theta$. Let $T_{k} \in \mathbb{R}_{++}$, $k \in \mathbb{N}$, be such that $T_{k} \rightarrow \infty$ and $\mathcal{L}\left(\left(\boldsymbol{\Delta}_{\boldsymbol{\theta}, T_{k}}, \boldsymbol{J}_{\boldsymbol{\theta}, T_{k}}\right) \mid \mathbb{P}_{\boldsymbol{\theta}, T_{k}}\right) \Rightarrow \mu_{\boldsymbol{\theta}}$ as $k \rightarrow \infty$. Then, for every $\boldsymbol{h}_{T_{k}} \in \mathbb{R}^{p}, \quad k \in \mathbb{N}$, with $\boldsymbol{h}_{T_{k}} \rightarrow \boldsymbol{h}$ as $k \rightarrow \infty$ and $\boldsymbol{\theta}+\boldsymbol{r}_{\boldsymbol{\theta}, T_{k}} \boldsymbol{h}_{T_{k}} \in \Theta$ for all $k \in \mathbb{N}$,

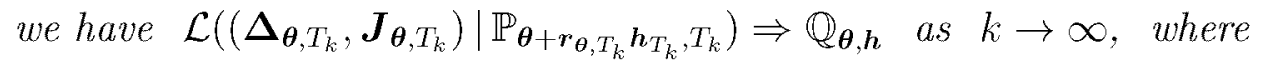

$$
\mathbb{Q}_{\boldsymbol{\theta}, \boldsymbol{h}}(B):=\int_{B} \exp \left\{\boldsymbol{h}^{\top} \boldsymbol{\Delta}-\frac{1}{2} \boldsymbol{h}^{\top} \boldsymbol{J} \boldsymbol{h}\right\} \mu_{\boldsymbol{\theta}}(\mathrm{d} \boldsymbol{\Delta}, \mathrm{d} \boldsymbol{J}), \quad B \in \mathcal{B}\left(\mathbb{R}^{p} \times \mathbb{R}^{p \times p}\right) .
$$

Consequently, the sequence $\left(X_{T_{k}}, \mathcal{X}_{T_{k}},\left\{\mathbb{P}_{\boldsymbol{\theta}+\boldsymbol{r}_{\boldsymbol{\theta}, T_{k}} \boldsymbol{h}, T_{k}}: \boldsymbol{h} \in \mathbb{R}^{p}\right\}\right)_{k \in \mathbb{N}}$ of statistical experiments converges to the statistical experiment $\left(\mathbb{R}^{p} \times \mathbb{R}^{p \times p}, \mathcal{B}\left(\mathbb{R}^{p} \times \mathbb{R}^{p \times p}\right),\left\{\mathbb{Q}_{\boldsymbol{\theta}, \boldsymbol{h}}: \boldsymbol{h} \in \mathbb{R}^{p}\right\}\right)$ as $k \rightarrow \infty$.

Note that for each $\boldsymbol{h} \in \mathbb{R}^{p}$, the probability measures $\mathbb{Q}_{\boldsymbol{\theta}, \boldsymbol{h}}$ and $\mathbb{Q}_{\boldsymbol{\theta}, \boldsymbol{0}}$ are equivalent, and

$$
\frac{\mathrm{d} \mathbb{Q}_{\boldsymbol{\theta}, \boldsymbol{h}}}{\mathrm{d} \mathbb{Q}_{\boldsymbol{\theta}, \boldsymbol{0}}}(\boldsymbol{\Delta}, \boldsymbol{J})=\exp \left\{\boldsymbol{h}^{\top} \boldsymbol{\Delta}-\frac{1}{2} \boldsymbol{h}^{\top} \boldsymbol{J} \boldsymbol{h}\right\}, \quad(\boldsymbol{\Delta}, \boldsymbol{J}) \in \mathbb{R}^{p} \times \mathbb{R}^{p \times p} .
$$

Proof. Let $(\Omega, \mathcal{A}, \mathbb{P})$ be a probability space and let $(\boldsymbol{\Delta}, \boldsymbol{J}): \Omega \rightarrow \mathbb{R}^{p} \times \mathbb{R}^{p \times p}$ be a measurable function such that $\mathcal{L}((\boldsymbol{\Delta}, \boldsymbol{J}) \mid \mathbb{P})=\mu_{\boldsymbol{\theta}}$. Using $\mathcal{L}\left(\left(\boldsymbol{\Delta}_{\boldsymbol{\theta}, T_{k}}, \boldsymbol{J}_{\boldsymbol{\theta}, T_{k}}\right) \mid \mathbb{P}_{\boldsymbol{\theta}, T_{k}}\right) \Rightarrow \mu_{\boldsymbol{\theta}}$ as $k \rightarrow \infty$, by Slutsky's lemma,

$$
\mathcal{L}\left(\frac{\mathrm{d} \mathbb{P}_{\boldsymbol{\theta}+\boldsymbol{r}_{\boldsymbol{\theta}, T_{k}} \boldsymbol{h}, T_{k}}}{\mathrm{~d} \mathbb{P}_{\boldsymbol{\theta}, T_{k}}} \mid \mathbb{P}_{\boldsymbol{\theta}, T_{k}}\right) \Rightarrow \mathcal{L}\left(\exp \left\{\boldsymbol{h}^{\top} \boldsymbol{\Delta}-\frac{1}{2} \boldsymbol{h}^{\top} \boldsymbol{J} \boldsymbol{h}\right\}\right) \quad \text { as } T \rightarrow \infty .
$$

By (2.1.3) and (2.1.4), applying Lemma 2.1.9, we conclude that the sequences $\left(\mathbb{P}_{\boldsymbol{\theta}+\boldsymbol{r}_{\boldsymbol{\theta}, T_{k}} \boldsymbol{h}, T_{k}}\right)_{k \in \mathbb{N}}$ and $\left(\mathbb{P}_{\boldsymbol{\theta}, T_{k}}\right)_{k \in \mathbb{N}}$ are mutually contiguous. Therefore, for each $\boldsymbol{h}, \boldsymbol{h}_{0} \in \mathbb{R}^{p}$, the probability of the set on which we have

$$
\log \frac{\mathrm{d} \mathbb{P}_{\boldsymbol{\theta}+\boldsymbol{r}_{\boldsymbol{\theta}, T_{k}} \boldsymbol{h , T _ { k }}}}{\mathrm{d} \mathbb{P}_{\boldsymbol{\theta}+\boldsymbol{r}_{\boldsymbol{\theta}, T_{k}} \boldsymbol{h}_{0}, T_{k}}}=\log \frac{\mathrm{d} \mathbb{P}_{\boldsymbol{\theta}+\boldsymbol{r}_{\boldsymbol{\theta}, T_{k}} \boldsymbol{h}, T_{k}}}{\mathrm{~d} \mathbb{P}_{\boldsymbol{\theta}, T_{k}}}-\log \frac{\mathrm{d} \mathbb{P}_{\boldsymbol{\theta}+\boldsymbol{r}_{\boldsymbol{\theta}, T_{k}} \boldsymbol{h}_{0}, T_{k}}}{\mathrm{~d} \mathbb{P}_{\boldsymbol{\theta}, T_{k}}}
$$


converges to one. By (2.1.1), one can obtain

$$
\log \frac{\mathrm{d} \mathbb{P}_{\boldsymbol{\theta}+\boldsymbol{r}_{\boldsymbol{\theta}, T_{k}} \boldsymbol{h}, T_{k}}}{\mathrm{~d} \mathbb{P}_{\boldsymbol{\theta}+\boldsymbol{r}_{\boldsymbol{\theta}, T_{k}} \boldsymbol{h}_{0}, T_{k}}}=\left(\boldsymbol{h}-\boldsymbol{h}_{0}\right)^{\top} \boldsymbol{\Delta}_{\boldsymbol{\theta}, T_{k}}-\frac{1}{2} \boldsymbol{h}^{\top} \boldsymbol{J}_{\boldsymbol{\theta}, T_{k}} \boldsymbol{h}+\frac{1}{2} \boldsymbol{h}_{0}^{\top} \boldsymbol{J}_{\boldsymbol{\theta}, T_{k}} \boldsymbol{h}_{0}+\mathrm{O}_{\mathbb{P}_{\boldsymbol{\theta}, T}}(1) \quad \text { as } T \rightarrow \infty .
$$

Hence it suffices to observe that $\mathcal{L}\left(\left(\boldsymbol{\Delta}_{\boldsymbol{\theta}, T_{k}}, \boldsymbol{J}_{\boldsymbol{\theta}, T_{k}}\right) \mid \mathbb{P}_{\boldsymbol{\theta}+\boldsymbol{r}_{\boldsymbol{\theta}, T_{k}} \boldsymbol{h}, T_{k}}\right) \Rightarrow \mathbb{Q}_{\boldsymbol{\theta}, \boldsymbol{h}}$ as $k \rightarrow \infty$ for all $h \in \mathbb{R}^{p}$ follows from Theorem 2.1.10.

The following statements are trivial consequences of Theorem 2.1.11, and they can also be derived from Theorems 9.4 and 9.8 of van der Vaart [46].

2.1.12 Proposition. Let $\Theta \subset \mathbb{R}^{p}$ be an open set. Let $\left(X_{T}, \mathcal{X}_{T},\left\{\mathbb{P}_{\boldsymbol{\theta}, T}: \boldsymbol{\theta} \in \Theta\right\}\right)_{T \in \mathbb{R}_{++}}$be a family of statistical experiments. Assume that $L A M N$ is satisfied at $\boldsymbol{\theta} \in \Theta$. Let $T_{k} \in \mathbb{R}_{++}$, $k \in \mathbb{N}$, be such that $\mathcal{L}\left(\left(\boldsymbol{\Delta}_{\boldsymbol{\theta}, T_{k}}, \boldsymbol{J}_{\boldsymbol{\theta}, T_{k}}\right) \mid \mathbb{P}_{\boldsymbol{\theta}, T_{k}}\right) \Rightarrow \mathcal{L}\left(\left(\eta_{\boldsymbol{\theta}} \mathcal{Z}, \eta_{\boldsymbol{\theta}} \eta_{\boldsymbol{\theta}}^{\top}\right) \mid \mathbb{P}\right)$ as $k \rightarrow \infty$, where $\mathcal{Z}:$ $\Omega \rightarrow \mathbb{R}^{p}$ and $\eta_{\boldsymbol{\theta}}: \Omega \rightarrow \mathbb{R}^{p \times p}$ are independent random elements on a probability space $(\Omega, \mathcal{F}, \mathbb{P})$ such that $\mathcal{L}(\mathcal{Z} \mid \mathbb{P})=\mathcal{N}_{p}\left(0, \boldsymbol{I}_{p}\right)$. Then, for every $\boldsymbol{h}_{T_{k}} \in \mathbb{R}^{p}, k \in \mathbb{N}$, with $\boldsymbol{h}_{T_{k}} \rightarrow \boldsymbol{h}$ as $k \rightarrow \infty$ and $\boldsymbol{\theta}+\boldsymbol{r}_{\boldsymbol{\theta}, T_{k}} \boldsymbol{h}_{T_{k}} \in \Theta$ for all $k \in \mathbb{N}$, we have $\mathcal{L}\left(\left(\boldsymbol{\Delta}_{\boldsymbol{\theta}, T_{k}}, \boldsymbol{J}_{\boldsymbol{\theta}, T_{k}}\right) \mid \mathbb{P}_{\boldsymbol{\theta}+\boldsymbol{r}_{\boldsymbol{\theta}, T_{k}} \boldsymbol{h}_{T_{k}}, T_{k}}\right) \Rightarrow \mathcal{L}\left(\left(\eta_{\boldsymbol{\theta}} \mathcal{Z}+\right.\right.$ $\left.\left.\eta_{\boldsymbol{\theta}} \eta_{\boldsymbol{\theta}}^{\top} \boldsymbol{h}, \eta_{\boldsymbol{\theta}} \eta_{\boldsymbol{\theta}}^{\top}\right) \mid \mathbb{P}\right)$ as $k \rightarrow \infty$. Consequently, the sequence $\left(X_{T_{k}}, \mathcal{X}_{T_{k}},\left\{\mathbb{P}_{\boldsymbol{\theta}+\boldsymbol{r}_{\boldsymbol{\theta}, T_{k}} \boldsymbol{h}, T_{k}}: \boldsymbol{h} \in\right.\right.$ $\left.\left.\mathbb{R}^{p}\right\}\right)_{T \in \mathbb{R}_{++}}$of statistical experiments converges to the statistical experiment $\left(\mathbb{R}^{p} \times \mathbb{R}^{p \times p}, \mathcal{B}\left(\mathbb{R}^{p} \times\right.\right.$ $\left.\left.\mathbb{R}^{p \times p}\right),\left\{\mathcal{L}\left(\left(\eta_{\boldsymbol{\theta}} \mathcal{Z}+\eta_{\boldsymbol{\theta}} \eta_{\boldsymbol{\theta}}^{\top} \boldsymbol{h}, \eta_{\boldsymbol{\theta}} \eta_{\boldsymbol{\theta}}^{\top}\right) \mid \mathbb{P}\right): \boldsymbol{h} \in \mathbb{R}^{p}\right\}\right)$ as $k \rightarrow \infty$.

2.1.13 Proposition. Let $\Theta \subset \mathbb{R}^{p}$ be an open set. Let $\left(X_{T}, \mathcal{X}_{T},\left\{\mathbb{P}_{\boldsymbol{\theta}, T}: \boldsymbol{\theta} \in \Theta\right\}\right)_{T \in \mathbb{R}_{++}}$be a family of statistical experiments. Assume that $L A N$ is satisfied at $\boldsymbol{\theta} \in \Theta$. Let $T_{k} \in$ $\mathbb{R}_{++}, \quad k \in \mathbb{N}$, be such that $\mathcal{L}\left(\left(\boldsymbol{\Delta}_{\boldsymbol{\theta}, T_{k}}, \boldsymbol{J}_{\boldsymbol{\theta}, T_{k}}\right) \mid \mathbb{P}_{\boldsymbol{\theta}, T_{k}}\right) \Rightarrow \mathcal{N}_{p}\left(0, \boldsymbol{J}_{\boldsymbol{\theta}}\right) \times \delta_{\boldsymbol{J}_{\boldsymbol{\theta}}}$ as $k \rightarrow \infty$ with some symmetric, strictly positive definite matrix $\boldsymbol{J}_{\boldsymbol{\theta}} \in \mathbb{R}^{p \times p}$. Then, for every $\boldsymbol{h}_{T_{k}} \in \mathbb{R}^{p}$, $k \in \mathbb{N}$, with $\boldsymbol{h}_{T_{k}} \rightarrow \boldsymbol{h}$ as $k \rightarrow \infty$ and $\boldsymbol{\theta}+\boldsymbol{r}_{\boldsymbol{\theta}, T_{k}} \boldsymbol{h}_{T_{k}} \in \Theta$ for all $k \in \mathbb{N}$, we have $\mathcal{L}\left(\left(\boldsymbol{\Delta}_{\boldsymbol{\theta}, T_{k}}, \boldsymbol{J}_{\boldsymbol{\theta}, T_{k}}\right) \mid \mathbb{P}_{\boldsymbol{\theta}+\boldsymbol{r}_{\boldsymbol{\theta}, T_{k}} \boldsymbol{h}_{T_{k}}, T_{k}}\right) \Rightarrow \mathcal{N}_{p}\left(\boldsymbol{J}_{\boldsymbol{\theta}} \boldsymbol{h}, \boldsymbol{J}_{\boldsymbol{\theta}}\right) \times \delta_{\boldsymbol{J}_{\boldsymbol{\theta}}}$ as $k \rightarrow \infty$. Consequently, the sequence $\left(X_{T_{k}}, \mathcal{X}_{T_{k}},\left\{\mathbb{P}_{\boldsymbol{\theta}+\boldsymbol{r}_{\boldsymbol{\theta}, T_{k}} \boldsymbol{h}, T_{k}}: \boldsymbol{h} \in \mathbb{R}^{p}\right\}\right)_{T \in \mathbb{R}_{++}}$of statistical experiments converges to the statistical experiment $\left(\mathbb{R}^{p}, \mathcal{B}\left(\mathbb{R}^{p}\right),\left\{\mathcal{N}_{p}\left(\boldsymbol{J}_{\boldsymbol{\theta}} \boldsymbol{h}, \boldsymbol{J}_{\boldsymbol{\theta}}\right): \boldsymbol{h} \in \mathbb{R}^{p}\right\}\right)$ as $k \rightarrow \infty$.

\subsection{Consequences of the local asymptotic properties}

The first and most important consequence is the following. If one can prove any of the local asymptotic properties of the likelihood function, get information for the asymptotic of the maximum likelihood estimator (MLE). If for a fixed $T \in \mathbb{R}_{++}$we have a continuous sample $X=(X(t))_{t \in[0, T]}$, maximizing $\log \frac{\mathrm{d \mathbb {P }}_{\boldsymbol{\theta}, T}}{\mathrm{dI}_{0, T}}(X)$ in $\boldsymbol{\theta}$ gives the MLE of $\boldsymbol{\theta}$ based on the observations $X$ denoted by $\widehat{\boldsymbol{\theta}}_{T}$. Then we have

$$
r_{\boldsymbol{\theta}, T}^{-1}\left(\widehat{\boldsymbol{\theta}}_{T}-\boldsymbol{\theta}\right)=\frac{\boldsymbol{\Delta}_{\boldsymbol{\theta}, T}}{\boldsymbol{J}_{\boldsymbol{\theta}, T}}
$$

and using the local asymptotic properties of the likelihood function and the continuous mapping theorem, we obtain the asymptotic behaviour of the MLE. For example in the case of LAN, 
asymptotic normality can be derived. For a detailed discussion see Section 3.2 .3 and Section 3.3 .3 .

The next consequence is connected to the question of efficiency. If LAMN property holds then we have the following local asymptotic minimax bound for arbitrary estimators, see, e.g., Le Cam and Yang [31, 6.6, Theorem 1] or Höpfner [23, Theorem 7.12].

2.2.1 Proposition. Let $\Theta \subset \mathbb{R}^{p}$ be an open set. Let $\left(X_{T}, \mathcal{X}_{T},\left\{\mathbb{P}_{\boldsymbol{\theta}, T}: \boldsymbol{\theta} \in \Theta\right\}\right)_{T \in \mathbb{R}_{+}}$be a family of statistical experiments. Assume that $L A M N$ is satisfied at $\boldsymbol{\theta} \in \Theta$. Let $T_{k} \in \mathbb{R}_{++}$, $k \in \mathbb{N}$, be such that $\mathcal{L}\left(\left(\boldsymbol{\Delta}_{\boldsymbol{\theta}, T_{k}}, \boldsymbol{J}_{\boldsymbol{\theta}, T_{k}}\right) \mid \mathbb{P}_{\boldsymbol{\theta}, T_{k}}\right) \Rightarrow \mathcal{L}\left(\left(\eta_{\boldsymbol{\theta}} \mathcal{Z}, \eta_{\boldsymbol{\theta}} \eta_{\boldsymbol{\theta}}^{\top}\right) \mid \mathbb{P}\right)$ as $k \rightarrow \infty$, where $\mathcal{Z}: \Omega \rightarrow \mathbb{R}^{p}$ and $\eta_{\theta}: \Omega \rightarrow \mathbb{R}^{p \times p}$ are independent random elements on a probability space $(\Omega, \mathcal{F}, \mathbb{P})$ such that $\mathcal{L}(\mathcal{Z} \mid \mathbb{P})=\mathcal{N}_{p}\left(0, \boldsymbol{I}_{p}\right)$. Let $w: \mathbb{R}^{p} \rightarrow \mathbb{R}_{+}$be a bowl-shaped loss function, i.e., for each $c \in \mathbb{R}_{+}$, the set $\left\{x \in \mathbb{R}^{p}: w(x) \leqslant c\right\}$ is closed, convex and symmetric. Then, for arbitrary estimators (statistics, i.e., measurable functions) $\widetilde{\boldsymbol{\theta}}_{T}: X_{T} \rightarrow \mathbb{R}^{p}, T \in \mathbb{R}_{+}$, of the parameter $\boldsymbol{\theta}$, one has

$$
\lim _{c \rightarrow \infty} \liminf _{k \rightarrow \infty} \sup _{\left\{x \in X_{T_{k}}:\left\|r_{\boldsymbol{\theta}, T_{k}}^{-1}\left(\widetilde{\boldsymbol{\theta}}_{T_{k}}(x)-\boldsymbol{\theta}\right)\right\| \leqslant c\right\}} \int_{X_{T_{k}}} w\left(r_{\boldsymbol{\theta}, T_{k}}^{-1}\left(\widetilde{\boldsymbol{\theta}}_{T_{k}}(x)-\boldsymbol{\theta}\right)\right) \mathbb{P}_{\boldsymbol{\theta}, T_{k}}(\mathrm{~d} x) \geqslant \mathbb{E}\left[w\left(\left(\eta_{\boldsymbol{\theta}}^{\top}\right)^{-1} \mathcal{Z}\right)\right] .
$$

Maximum likelihood estimators attain this bound for bounded loss function $w$, see, e.g., Le Cam and Yang [31, 6.6, Remark 11]. Moreover, maximum likelihood estimators are asymptotically efficient in Hájek's convolution theorem sense (for example, see, Le Cam and Yang $[31,6.6$, Theorem 3 and Remark 13]; Jeganathan [26]).

The local asymptotic properties of the likelihood function are useful in hypothesis testing as well.

2.2.2 Definition. $A$ (randomized) test (function) in a statistical experiment $\left(X, \mathcal{X},\left\{\mathbb{P}_{\boldsymbol{\theta}}: \boldsymbol{\theta} \in\right.\right.$ $\Theta\}$ ) is a Borel measurable function $\phi: X \rightarrow[0,1]$. (The interpretation is that if $x \in X$ is observed, then a null hypothesis $H_{0} \subset \Theta$ is rejected with probability $\phi(x)$.)

The power function of a test $\phi$ is the function $\theta \mapsto \int_{X} \phi(x) \mathbb{P}_{\boldsymbol{\theta}}(\mathrm{d} x)$. (This gives the probability that the null hypothesis $H_{0}$ is rejected.)

For $\alpha \in(0,1)$, a test $\phi$ is of level $\alpha$ for testing a null hypothesis $H_{0}$ if

$$
\sup \left\{\int_{X} \phi(x) \mathbb{P}_{\boldsymbol{\theta}}(\mathrm{d} x): \theta \in H_{0}\right\} \leqslant \alpha .
$$

If the LAN property holds then one obtains asymptotically optimal tests in the following way, see, e.g., Theorem 15.4 and Addendum 15.5 of van der Vaart [46].

2.2.3 Theorem. Let $\Theta \subset \mathbb{R}^{p}$ be an open set. Let $\left(X_{T}, \mathcal{X}_{T},\left\{\mathbb{P}_{\boldsymbol{\theta}, T}: \boldsymbol{\theta} \in \Theta\right\}\right)_{T \in \mathbb{R}_{++}}$be a family of statistical experiments such that $L A N$ is satisfied at $\boldsymbol{\theta}_{0} \in \Theta$. Let $T_{k} \in \mathbb{R}_{++}, k \in \mathbb{N}$, be such that $\mathcal{L}\left(\left(\boldsymbol{\Delta}_{\boldsymbol{\theta}_{0}, T_{k}}, \boldsymbol{J}_{\boldsymbol{\theta}_{0}, T_{k}}\right) \mid \mathbb{P}_{\boldsymbol{\theta}_{0}, T_{k}}\right) \Rightarrow \mathcal{N}_{p}\left(\mathbf{0}, \boldsymbol{J}_{\boldsymbol{\theta}_{0}}\right) \times \delta_{\boldsymbol{J}_{\boldsymbol{\theta}_{0}}}$ as $k \rightarrow \infty$ with some symmetric, strictly positive definite matrix $\boldsymbol{J}_{\boldsymbol{\theta}_{0}} \in \mathbb{R}^{p \times p}$. Let $\psi: \Theta \rightarrow \mathbb{R}$ be differentiable at $\boldsymbol{\theta}_{0} \in \Theta$ with 
$\psi\left(\boldsymbol{\theta}_{0}\right)=0$ and $\psi^{\prime}\left(\boldsymbol{\theta}_{0}\right) \neq 0$. Let $\alpha \in(0,1)$. For each $k \in \mathbb{N}$, let $\phi_{k}: X_{T_{k}} \rightarrow[0,1]$ be a test of level $\alpha$ for testing $H_{0}: \psi(\boldsymbol{\theta}) \leqslant 0$ against $H_{1}: \psi(\boldsymbol{\theta})>0$, i.e., it is a Borel measurable function such that

$$
\sup \left\{\int_{X_{T_{k}}} \phi_{k}(x) \mathbb{P}_{\boldsymbol{\theta}, T_{k}}(\mathrm{~d} x): \boldsymbol{\theta} \in \Theta, \psi(\boldsymbol{\theta}) \leqslant 0\right\} \leqslant \alpha .
$$

Then for each $\boldsymbol{h} \in \mathbb{R}^{p}$ with $\left\langle\psi^{\prime}\left(\boldsymbol{\theta}_{0}\right), \boldsymbol{h}\right\rangle>0$, the power function of the test $\phi_{k}$ satisfies

$$
\limsup _{k \rightarrow \infty} \int_{X_{T_{k}}} \phi_{k}(x) \mathbb{P}_{\boldsymbol{\theta}_{0}+\boldsymbol{r}_{\boldsymbol{\theta}_{0}, T_{k}} \boldsymbol{h}, T_{k}}(\mathrm{~d} x) \leqslant 1-\Phi\left(z_{\alpha}-\frac{\left\langle\psi^{\prime}\left(\boldsymbol{\theta}_{0}\right), \boldsymbol{h}\right\rangle}{\sqrt{\left\langle\boldsymbol{J}_{\boldsymbol{\theta}_{0}}^{-1} \psi^{\prime}\left(\boldsymbol{\theta}_{0}\right), \psi^{\prime}\left(\boldsymbol{\theta}_{0}\right)\right\rangle}}\right),
$$

where $\Phi$ denotes the standard normal distribution function, and $z_{\alpha}$ denotes the upper $\alpha$ quantile of the standard normal distribution.

Moreover, if $S_{\boldsymbol{\theta}_{0}, k}: X_{T_{k}} \rightarrow \mathbb{R}, k \in \mathbb{N}$, are Borel measurable functions such that

$$
S_{\boldsymbol{\theta}_{0}, k}=\frac{\left\langle\boldsymbol{J}_{\boldsymbol{\theta}_{0}}^{-1} \boldsymbol{\Delta}_{\boldsymbol{\theta}_{0}, T_{k}}, \psi^{\prime}\left(\boldsymbol{\theta}_{0}\right)\right\rangle}{\sqrt{\left\langle\boldsymbol{J}_{\boldsymbol{\theta}_{0}}^{-1} \psi^{\prime}\left(\boldsymbol{\theta}_{0}\right), \psi^{\prime}\left(\boldsymbol{\theta}_{0}\right)\right\rangle}}+\mathrm{o}_{\mathbb{P}_{\boldsymbol{\theta}_{0}, k}}(1), \quad k \in \mathbb{N}
$$

then the family of tests that reject for values $S_{\boldsymbol{\theta}_{0}, k}$ exceeding $z_{\alpha}$ is asymptotically optimal for testing $H_{0}: \psi(\boldsymbol{\theta}) \leqslant 0$ against $H_{1}: \psi(\boldsymbol{\theta})>0$ in the sense that for every $\boldsymbol{h} \in \mathbb{R}^{p}$ with $\left\langle\psi^{\prime}\left(\boldsymbol{\theta}_{0}\right), \boldsymbol{h}\right\rangle>0$,

$$
\mathbb{P}\left(S_{\boldsymbol{\theta}_{0}, k}(x) \geqslant z_{\alpha}\right) \rightarrow 1-\Phi\left(z_{\alpha}-\frac{\left\langle\psi^{\prime}\left(\boldsymbol{\theta}_{0}, \boldsymbol{h}\right\rangle\right.}{\sqrt{\left\langle\boldsymbol{J}_{\boldsymbol{\theta}_{0}}^{-1} \psi^{\prime}\left(\boldsymbol{\theta}_{0}\right), \psi^{\prime}\left(\boldsymbol{\theta}_{0}\right)\right\rangle}}\right) \quad \text { as } k \rightarrow \infty .
$$

\subsection{An example for a Heston model}

\subsubsection{A short introduction and preliminaries}

Heston models have been extensively used in financial mathematics since one can well-fit them to real financial data set, and they are well-tractable from the point of view of computability as well, see Heston [22]. These models have been investigated with many statistical techniques. In the introduction of Barczy and Pap [7] one can find a detailed overview on the topic of parameter estimation, e.g. parameter estimation on discrete and continuous time observations, maximum likelihood estimator, quasi-maximum likelihood procedure and (weighted) conditional least squares estimator. In this section the local asymptotic properties of the likelihood ratios are studied.

Let us consider a Heston model

$$
\left\{\begin{array}{l}
\mathrm{d} Y_{t}=\left(a-b Y_{t}\right) \mathrm{d} t+\sigma_{1} \sqrt{Y_{t}} \mathrm{~d} W_{t}, \\
\mathrm{~d} X_{t}=\left(\alpha-\beta Y_{t}\right) \mathrm{d} t+\sigma_{2} \sqrt{Y_{t}}\left(\varrho \mathrm{d} W_{t}+\sqrt{1-\varrho^{2}} \mathrm{~d} B_{t}\right), \quad t \geqslant 0,
\end{array} \quad t\right.
$$


where $a>0, b, \alpha, \beta \in \mathbb{R}, \sigma_{1}>0, \sigma_{2}>0, \varrho \in(-1,1)$ and $\left(W_{t}, B_{t}\right)_{t \geqslant 0}$ is a 2-dimensional standard Wiener process. Here one can interpret $X_{t}$ as the log-price of an asset, and $Y_{t}$ as the stochastic volatility of the asset price at time $t \geqslant 0$. The squared volatility process $\left(\sigma_{2}^{2} Y_{t}\right)_{t \geqslant 0}$ is a Cox-Ingersoll-Ross (CIR) process. The parameter $\sigma_{1}$ is the so-called volatility of the volatility, and $\varrho$ is the correlation between the driving standard Wiener processes $\left(W_{t}\right)_{t \geq 0}$ and $\left(\varrho \mathrm{d} W_{t}+\sqrt{1-\varrho^{2}} \mathrm{~d} B_{t}\right)_{t \geq 0}$. One can distinguish three cases: subcritical if $b>0$, critical if $b=0$ and supercritical if $b<0$. We study local asymptotic properties of the likelihood ratios of the model (2.3.1) concerning the drift parameter $(a, \alpha, b, \beta)$. The parameters $\sigma_{1}, \sigma_{2}$ and $\varrho$ are supposed to be known, because using continuous time observations these parameters can be calculated almost surely, see Barczy and Pap ([7, Remark 2.6]).

The original Heston model (see Heston [22]) takes the form

$$
\left\{\begin{array}{l}
\mathrm{d} Y_{t}=\kappa\left(\theta-Y_{t}\right) \mathrm{d} t+\sigma \sqrt{Y_{t}} \mathrm{~d} W_{t}, \\
\mathrm{~d} X_{t}=\mu S_{t} \mathrm{~d} t+S_{t} \sqrt{Y_{t}}\left(\varrho \mathrm{d} W_{t}+\sqrt{1-\varrho^{2}} \mathrm{~d} B_{t}\right),
\end{array} \quad t \geqslant 0,\right.
$$

where $\left(X_{t}\right)_{t \geq 0}$ is the price process of an asset, $\mu \in \mathbb{R}$ is the rate of return of the asset, $\theta>0$ is the so-called long variance (long run average price variance, i.e., the limit of $\mathbb{E}\left(Y_{t}\right)$ as $t \rightarrow \infty), \kappa>0$ is the rate at which $\left(Y_{t}\right)_{t \geq 0}$ reverts to $\theta$, and $\sigma>0$ is the so-called volatility of volatility. We call the attention that there are two differences between the models (2.3.1) and (2.3.2). Namely, in (2.3.2) the coefficient $\kappa$ can only be positive, while in (2.3.1) the corresponding coefficient $b$ can be an arbitrary real number. In other words, the first coordinate process in (2.3.2) can be subcritical, critical or supercritical, but in (2.3.2) it can only be subcritical. Morover, the second coordinate process in $(2.3 .2)$ is the price process, while in (2.3.1) it is the log-price process.

In case of the one-dimensional CIR process $Y$, Overbeck [36] examined local asymptotic properties of the likelihood ratios concerning the drift parameter $(a, b)$, and proved the following results under the assumption $a \in\left(\frac{\sigma_{1}^{2}}{2}, \infty\right)$, which guarantees that the information matrix process tends to infinity almost surely. It turned out LAN is valid in the subcritical case. In the critical case LAN has been proved for the submodel when $b=0$ is known, and only LAQ has been shown for the submodel when $a \in\left(\frac{\sigma_{1}^{2}}{2}, \infty\right)$ is known, but the asymptotic property of the experiment locally at $(a, 0)$ with a suitable two-dimensional localization sequence remained as an open question. In the supercritical case LAMN has been proved for the submodel when $a \in\left(\frac{\sigma_{1}^{2}}{2}, \infty\right)$ is known. Kutoyants [30] investigated statistical inference for 1-dimensional ergodic diffusion processes. LAN for the one-dimensional CIR process $Y$ in the subcritical case also follows from his general result (see Proposition 2.2).

For the Heston model (2.3.1), we assume again $a \in\left(\frac{\sigma_{1}^{2}}{2}, \infty\right)$. We prove LAN in the subcritical case (see Theorem 2.3.7), LAQ in the critical case (see Theorem 2.3.10), and is shown that LAQ does not hold in the supercritical case, although we can describe the asymptotic property of the experiment locally at $(a, \alpha, b, \beta)$ with a suitable four-dimensional degenerate localization sequence (see Theorem 2.3.12). In the critical case LAN will be shown for the submodel when $b=0$ and $\beta \in \mathbb{R}$ are known (see Theorem 2.3.10). In the supercritical 
case LAMN will be proved for the submodel when $a \in\left(\frac{\sigma_{1}^{2}}{2}, \infty\right)$ and $\alpha \in \mathbb{R}$ are known (see Theorem 2.3.12).

If the LAN property holds then one can obtain asymptotically optimal tests (see Remarks 2.3.9 and 2.3.11) based on Theorem 15.4 and Addendum 15.5 of van der Vaart [46]. If the LAMN property holds then exists a local asymptotic minimax bound for arbitrary estimators, see, e.g., Le Cam and Yang [31, 6.6, Theorem 1] or Höpfner [23, Theorem 7.12]. Moreover, any maximum likelihood estimator attains this bound for bounded loss function (see Le Cam and Yang [31, 6.6, Remark 11]), and it is asymptotically efficient in Hájek's convolution theorem sense (for example, see, Le Cam and Yang [31, 6.6, Theorem 3 and Remark 13]; Jeganathan [26]). Asymptotic behaviour of maximum likelihood estimators are described in all cases in Barczy and Pap [7].

The next proposition is about the existence and uniqueness of a strong solution of the SDE (2.3.1), see, e.g., Barczy and Pap [7, Proposition 2.1].

2.3.1 Proposition. Let $(\Omega, \mathcal{F}, \mathbb{P})$ be a probability space. Let $\left(W_{t}, B_{t}\right)_{t \in \mathbb{R}_{+}}$be a D-dimensional standard Wiener process. Let $\left(\eta_{0}, \zeta_{0}\right)$ be a random vector independent of $\left(W_{t}, B_{t}\right)_{t \in \mathbb{R}_{+}}$ satisfying $\mathbb{P}\left(\eta_{0} \in \mathbb{R}_{+}\right)=1$. Then for all $a \in \mathbb{R}_{++}, \quad b, \alpha, \beta \in \mathbb{R}, \sigma_{1}, \sigma_{2} \in \mathbb{R}_{++}$, $\varrho \in(-1,1)$, there is a (pathwise) unique strong solution $\left(Y_{t}, X_{t}\right)_{t \in \mathbb{R}_{+}}$of the SDE (2.3.1) such that $\mathbb{P}\left(\left(Y_{0}, X_{0}\right)=\left(\eta_{0}, \zeta_{0}\right)\right)=1$ and $\mathbb{P}\left(Y_{t} \in \mathbb{R}_{+}\right.$for all $\left.t \in \mathbb{R}_{+}\right)=1$. Further, for all $s, t \in \mathbb{R}_{+}$with $s \leqslant t$,

$$
\left\{\begin{array}{l}
Y_{t}=\mathrm{e}^{-b(t-s)}\left(Y_{s}+a \int_{s}^{t} \mathrm{e}^{-b(s-u)} \mathrm{d} u+\sigma_{1} \int_{s}^{t} \mathrm{e}^{-b(s-u)} \sqrt{Y_{u}} \mathrm{~d} W_{u}\right) \\
X_{t}=X_{s}+\int_{s}^{t}\left(\alpha-\beta Y_{u}\right) \mathrm{d} u+\sigma_{2} \int_{s}^{t} \sqrt{Y_{u}}\left(\varrho \mathrm{d} W_{u}+\sqrt{1-\varrho^{2}} \mathrm{~d} B_{u}\right) .
\end{array}\right.
$$

Next we present a result about the first moment of $\left(Y_{t}, X_{t}\right)_{t \in \mathbb{R}_{+}}$, see Barczy and Pap [7]. We note that Hurn et al. [24, Equation (23)] derived the same formula for the expectation of $\left(Y_{t}, X_{t}\right), t \in \mathbb{R}_{+}$, by a different method. Note also that the formula for $\mathbb{E}\left(Y_{t}\right), t \in \mathbb{R}_{+}$, is well-known.

2.3.2 Proposition. Let $\left(Y_{t}, X_{t}\right)_{t \in \mathbb{R}_{+}}$be the unique strong solution of the $S D E(2.3 .1)$ satisfying $\mathbb{P}\left(Y_{0} \in \mathbb{R}_{+}\right)=1$ and $\mathbb{E}\left(Y_{0}\right)<\infty, \mathbb{E}\left(\left|X_{0}\right|\right)<\infty$. Then

$$
\left[\begin{array}{l}
\mathbb{E}\left(Y_{t}\right) \\
\mathbb{E}\left(X_{t}\right)
\end{array}\right]=\left[\begin{array}{cc}
\mathrm{e}^{-b t} & 0 \\
-\beta \int_{0}^{t} \mathrm{e}^{-b u} \mathrm{~d} u & 1
\end{array}\right]\left[\begin{array}{l}
\mathbb{E}\left(Y_{0}\right) \\
\mathbb{E}\left(X_{0}\right)
\end{array}\right]+\left[\begin{array}{cc}
\int_{0}^{t} \mathrm{e}^{-b u} \mathrm{~d} u & 0 \\
-\beta \int_{0}^{t}\left(\int_{0}^{u} \mathrm{e}^{-b v} \mathrm{~d} v\right) \mathrm{d} u & t
\end{array}\right]\left[\begin{array}{l}
a \\
\alpha
\end{array}\right], \quad t \in \mathbb{R}_{+} .
$$

Consequently, if $b \in \mathbb{R}_{++}$, then

$$
\lim _{t \rightarrow \infty} \mathbb{E}\left(Y_{t}\right)=\frac{a}{b}, \quad \lim _{t \rightarrow \infty} t^{-1} \mathbb{E}\left(X_{t}\right)=\alpha-\frac{\beta a}{b},
$$

if $b=0$, then

$$
\lim _{t \rightarrow \infty} t^{-1} \mathbb{E}\left(Y_{t}\right)=a, \quad \lim _{t \rightarrow \infty} t^{-2} \mathbb{E}\left(X_{t}\right)=-\frac{1}{2} \beta a,
$$


if $b \in \mathbb{R}_{--}$, then

$$
\lim _{t \rightarrow \infty} e^{b t} \mathbb{E}\left(Y_{t}\right)=\mathbb{E}\left(Y_{0}\right)-\frac{a}{b}, \quad \lim _{t \rightarrow \infty} e^{b t} \mathbb{E}\left(X_{t}\right)=\frac{\beta}{b} \mathbb{E}\left(Y_{0}\right)-\frac{\beta a}{b^{2}}
$$

Based on the asymptotic behaviour of the expectations $\left(\mathbb{E}\left(Y_{t}\right), \mathbb{E}\left(X_{t}\right)\right)$ as $t \rightarrow \infty$, one can classify Heston processes given by the SDE (2.3.1).

2.3.3 Definition. Let $\left(Y_{t}, X_{t}\right)_{t \in \mathbb{R}_{+}}$be the unique strong solution of the SDE (2.3.1) satisfying $\mathbb{P}\left(Y_{0} \in \mathbb{R}_{+}\right)=1$. We call $\left(Y_{t}, X_{t}\right)_{t \in \mathbb{R}_{+}}$subcritical, critical or supercritical if $b \in \mathbb{R}_{++}, b=0$ or $b \in \mathbb{R}_{--}$, respectively.

The following result states ergodicity of the process $\left(Y_{t}\right)_{t \in \mathbb{R}_{+}}$given by the first equation in (2.3.1) in the subcritical case, see, e.g., Cox et al. [13, Equation (20)], Li and Ma [32, Theorem 2.6] or Theorem 4.1 in Barczy et al. [6].

2.3.4 Theorem. Let $a, b, \sigma_{1} \in \mathbb{R}_{++}$. Let $\left(Y_{t}\right)_{t \in \mathbb{R}_{+}}$be the unique strong solution of the first equation of the $S D E(2.3 .1)$ satisfying $\mathbb{P}\left(Y_{0} \in \mathbb{R}_{+}\right)=1$.

(i) Then $Y_{t} \stackrel{\mathcal{D}}{\longrightarrow} Y_{\infty}$ as $t \rightarrow \infty$, and the distribution of $Y_{\infty}$ is given by

$$
\mathbb{E}\left(\mathrm{e}^{-\lambda Y_{\infty}}\right)=\left(1+\frac{\sigma_{1}^{2}}{2 b} \lambda\right)^{-2 a / \sigma_{1}^{2}}, \quad \lambda \in \mathbb{R}_{+},
$$

i.e., $Y_{\infty}$ has Gamma distribution with parameters $2 a / \sigma_{1}^{2}$ and $2 b / \sigma_{1}^{2}$, hence

$$
\mathbb{E}\left(Y_{\infty}^{\kappa}\right)=\frac{\Gamma\left(\frac{2 a}{\sigma_{1}^{2}}+\kappa\right)}{\left(\frac{2 b}{\sigma_{1}^{2}}\right)^{\kappa} \Gamma\left(\frac{2 a}{\sigma_{1}^{2}}\right)}, \quad \kappa \in\left(-\frac{2 a}{\sigma_{1}^{2}}, \infty\right) .
$$

Especially, $\mathbb{E}\left(Y_{\infty}\right)=\frac{a}{b}$. Further, if $a \in\left(\frac{\sigma_{1}^{2}}{2}, \infty\right)$, then $\mathbb{E}\left(\frac{1}{Y_{\infty}}\right)=\frac{2 b}{2 a-\sigma_{1}^{2}}$.

(ii) For all Borel measurable functions $f: \mathbb{R} \rightarrow \mathbb{R}$ such that $\mathbb{E}\left(\left|f\left(Y_{\infty}\right)\right|\right)<\infty$, we have

$$
\frac{1}{T} \int_{0}^{T} f\left(Y_{s}\right) \mathrm{d} s \stackrel{\text { a.s. }}{\longrightarrow} \mathbb{E}\left(f\left(Y_{\infty}\right)\right) \quad \text { as } T \rightarrow \infty .
$$

\subsubsection{Radon-Nikodym derivatives for Heston models}

From this section, we consider the Heston model $(2.3 .1)$ with fixed $\sigma_{1}, \sigma_{2} \in \mathbb{R}_{++}, \varrho \in(-1,1)$, and fixed initial value $\left(Y_{0}, X_{0}\right)=\left(y_{0}, x_{0}\right) \in \mathbb{R}_{++} \times \mathbb{R}$, and we consider $\boldsymbol{\theta}:=(a, \alpha, b, \beta) \in$ $\mathbb{R}_{++} \times \mathbb{R}^{3}=: \Theta$ as a parameter. Note that $\Theta \subset \mathbb{R}^{4}$ is an open subset.

Let $\mathbb{P}_{\boldsymbol{\theta}}$ denote the probability measure induced by $\left(Y_{t}, X_{t}\right)_{t \in \mathbb{R}_{+}}$on the measurable space $\left(C\left(\mathbb{R}_{+}, \mathbb{R}^{2}\right), \mathcal{B}\left(C\left(\mathbb{R}_{+}, \mathbb{R}^{2}\right)\right)\right)$ endowed with the natural filtration $\left(\mathcal{G}_{t}\right)_{t \in \mathbb{R}_{+}}$, given by $\mathcal{G}_{t}:=$ $\varphi_{t}^{-1}\left(\mathcal{B}\left(C\left(\mathbb{R}_{+}, \mathbb{R}^{2}\right)\right)\right), t \in \mathbb{R}_{+}$, where $\varphi_{t}: C\left(\mathbb{R}_{+}, \mathbb{R}^{2}\right) \rightarrow C\left(\mathbb{R}_{+}, \mathbb{R}^{2}\right)$ is the mapping $\varphi_{t}(f)(s):=$ 
$f(t \wedge s), s, t \in \mathbb{R}_{+}, \quad f \in C\left(\mathbb{R}_{+}, \mathbb{R}^{2}\right)$. For all $T \in \mathbb{R}_{++}$, let $\mathbb{P}_{\boldsymbol{\theta}, T}:=\left.\mathbb{P}_{\boldsymbol{\theta}}\right|_{\mathcal{G}_{T}}$ be the restriction of $\mathbb{P}_{\boldsymbol{\theta}}$ to $\mathcal{G}_{T}$.

Write the Heston model (2.3.1) in the form

$$
\left[\begin{array}{l}
\mathrm{d} Y_{t} \\
\mathrm{~d} X_{t}
\end{array}\right]=\left[\begin{array}{l}
a-b Y_{t} \\
\alpha-\beta Y_{t}
\end{array}\right] \mathrm{d} t+\sqrt{Y_{t}}\left[\begin{array}{cc}
\sigma_{1} & 0 \\
\sigma_{2} \varrho & \sigma_{2} \sqrt{1-\varrho^{2}}
\end{array}\right]\left[\begin{array}{l}
\mathrm{d} W_{t} \\
\mathrm{~d} B_{t}
\end{array}\right] .
$$

In order to calculate Radon-Nikodym derivatives $\frac{d \mathbb{P}_{\widetilde{\boldsymbol{\theta}}}, T}{d \mathbb{P}_{\boldsymbol{\theta}, T}}$ for certain $\boldsymbol{\theta}, \widetilde{\boldsymbol{\theta}} \in \Theta$, we need the following statement, which can be derived from formula (7.139) in Section 7.6.4 of Liptser and Shiryaev [33], see Barczy and Pap [7, Lemma 3.1].

2.3.5 Lemma. Let $a, \widetilde{a} \in\left[\frac{\sigma_{1}^{2}}{2}, \infty\right)$ and $b, \widetilde{b}, \alpha, \widetilde{\alpha}, \beta, \widetilde{\beta} \in \mathbb{R}$. Let $\boldsymbol{\theta}:=(a, \alpha, b, \beta)$ and $\widetilde{\boldsymbol{\theta}}:=(\widetilde{a}, \widetilde{\alpha}, \widetilde{b}, \widetilde{\beta})$. Then for all $T \in \mathbb{R}_{++}$, the measures $\mathbb{P}_{\boldsymbol{\theta}, T}$ and $\mathbb{P}_{\widetilde{\boldsymbol{\theta}}, T}$ are absolutely continuous with respect to each other, and

$$
\begin{aligned}
\log \frac{\mathrm{d} \mathbb{P}_{\widetilde{\boldsymbol{\theta}}, T}}{\mathrm{~d} \mathbb{P}_{\boldsymbol{\theta}, T}}(Y, X)= & \int_{0}^{T} \frac{1}{Y_{s}}\left[\begin{array}{c}
\left(\widetilde{a}-\widetilde{b} Y_{s}\right)-\left(a-b Y_{s}\right) \\
\left(\widetilde{\alpha}-\widetilde{\beta} Y_{s}\right)-\left(\alpha-\beta Y_{s}\right)
\end{array}\right]^{\top} \boldsymbol{S}^{-1}\left[\begin{array}{c}
\mathrm{d} Y_{s} \\
\mathrm{~d} X_{s}
\end{array}\right] \\
& -\frac{1}{2} \int_{0}^{T} \frac{1}{Y_{s}}\left[\begin{array}{c}
\left(\widetilde{a}-\widetilde{b} Y_{s}\right)-\left(a-b Y_{s}\right) \\
\left(\widetilde{\alpha}-\widetilde{\beta} Y_{s}\right)-\left(\alpha-\beta Y_{s}\right)
\end{array}\right]^{\top} \boldsymbol{S}^{-1}\left[\begin{array}{c}
\left(\widetilde{a}-\widetilde{b} Y_{s}\right)+\left(a-b Y_{s}\right) \\
\left(\widetilde{\alpha}-\widetilde{\beta} Y_{s}\right)+\left(\alpha-\beta Y_{s}\right)
\end{array}\right] \mathrm{d} s,
\end{aligned}
$$

where

$$
\boldsymbol{S}:=\left[\begin{array}{cc}
\sigma_{1} & 0 \\
\sigma_{2} \varrho & \sigma_{2} \sqrt{1-\varrho^{2}}
\end{array}\right]\left[\begin{array}{cc}
\sigma_{1} & \sigma_{2} \varrho \\
0 & \sigma_{2} \sqrt{1-\varrho^{2}}
\end{array}\right]=\left[\begin{array}{cc}
\sigma_{1}^{2} & \varrho \sigma_{1} \sigma_{2} \\
\varrho \sigma_{1} \sigma_{2} & \sigma_{2}^{2}
\end{array}\right]
$$

Moreover, the process

$$
\left(\frac{\mathrm{d} \mathbb{P}_{\widetilde{\boldsymbol{\theta}}, T}}{\mathrm{~d} \mathbb{P}_{\boldsymbol{\theta}, T}}\right)_{T \in \mathbb{R}_{+}}
$$

is a $\mathbb{P}_{\boldsymbol{\theta}}$-martingale with respect to the filtration $\left(\mathcal{G}_{T}\right)_{T \in \mathbb{R}_{+}}$.

The martingale property of the process (2.3.8) is a consequence of Theorem 3.4 in Chapter III of Jacod and Shiryaev [25].

Introduce the family

$$
\left(\mathcal{E}_{T}\right)_{T \in \mathbb{R}_{++}}:=\left(C\left(\mathbb{R}_{+}, \mathbb{R}^{2}\right), \mathcal{B}\left(C\left(\mathbb{R}_{+}, \mathbb{R}^{2}\right)\right),\left\{\mathbb{P}_{\boldsymbol{\theta}, T}: \boldsymbol{\theta} \in \mathbb{R}_{++} \times \mathbb{R}^{3}\right\}\right)_{T \in \mathbb{R}_{++}}
$$

of statistical experiments. Since the log-likelihood function is a quadratic polynomial of the parameters, $(2.3 .9)$ is a so-called $(\gamma, \Gamma ; \varphi)$-model, see, e.g., Shiryaev and Spokoiny [44]. In order to investigate convergence of the family (2.3.9), we derive the following corollary. 
2.3.6 Corollary. Let $a \in\left[\frac{\sigma_{1}^{2}}{2}, \infty\right), b, \alpha, \beta, \in \mathbb{R}$ and $T \in \mathbb{R}_{++}$. Put $\boldsymbol{\theta}:=(a, \alpha, b, \beta)$. If

$$
\boldsymbol{r}_{\boldsymbol{\theta}, T}=\left[\begin{array}{cccc}
r_{\boldsymbol{\theta}, T, 1} & 0 & 0 & 0 \\
0 & r_{\boldsymbol{\theta}, T, 2} & 0 & 0 \\
0 & 0 & r_{\boldsymbol{\theta}, T, 3} & 0 \\
0 & 0 & 0 & r_{\boldsymbol{\theta}, T, 4}
\end{array}\right] \in \mathbb{R}^{4 \times 4}, \quad \boldsymbol{h}_{T}=\left[\begin{array}{c}
h_{T, 1} \\
h_{T, 2} \\
h_{T, 3} \\
h_{T, 4}
\end{array}\right] \in \mathbb{R}^{4}
$$

such that $a+r_{\boldsymbol{\theta}, T, 1} h_{T, 1} \in\left[\frac{\sigma_{1}^{2}}{2}, \infty\right)$, then

$$
\log \frac{\mathrm{d} \mathbb{P}_{\boldsymbol{\theta}+\boldsymbol{r}_{\boldsymbol{\theta}, T} \boldsymbol{h}_{T}, T}}{\mathrm{d \mathbb {P } _ { \boldsymbol { \theta } , T }}}(Y, X)=\boldsymbol{h}_{T}^{\top} \boldsymbol{\Delta}_{\boldsymbol{\theta}, T}(Y, X)-\frac{1}{2} \boldsymbol{h}_{T}^{\top} \boldsymbol{J}_{\boldsymbol{\theta}, T}(Y, X) \boldsymbol{h}_{T},
$$

where

$$
\boldsymbol{\Delta}_{\boldsymbol{\theta}, T}(Y, X):=\boldsymbol{r}_{\boldsymbol{\theta}, T}\left(\boldsymbol{I}_{2} \otimes\left[\begin{array}{cc}
\sigma_{1} & \sigma_{2} \varrho \\
0 & \sigma_{2} \sqrt{1-\varrho^{2}}
\end{array}\right]^{-1}\right)\left[\begin{array}{c}
\int_{0}^{T} \frac{\mathrm{d} W_{s}}{\sqrt{Y_{s}}} \\
\int_{0}^{T} \frac{\mathrm{d} B_{s}}{\sqrt{Y_{s}}} \\
-\int_{0}^{T} \sqrt{Y_{s}} \mathrm{~d} W_{s} \\
-\int_{0}^{T} \sqrt{Y_{s}} \mathrm{~d} B_{s}
\end{array}\right]
$$

and

$$
\boldsymbol{J}_{\boldsymbol{\theta}, T}(Y, X):=\boldsymbol{r}_{\boldsymbol{\theta}, T}\left(\left[\begin{array}{cc}
\int_{0}^{T} \frac{\mathrm{d} s}{Y_{s}} & -T \\
-T & \int_{0}^{T} Y_{s} \mathrm{~d} s
\end{array}\right] \otimes \boldsymbol{S}^{-1}\right) \boldsymbol{r}_{\boldsymbol{\theta}, T},
$$

where $\boldsymbol{A} \otimes \boldsymbol{B}$ denotes the Kronecker product of matrices $\boldsymbol{A}$ and $\boldsymbol{B}$. Consequently, by Remark 2.1.3, the quadratic approximation (2.1.1) is valid.

Proof. Using equations (2.3.6), one can get

$$
\begin{aligned}
\log \frac{\mathrm{d} \mathbb{P}_{\widetilde{\boldsymbol{\theta}}, T}}{\mathrm{~d} \mathbb{P}_{\boldsymbol{\theta}, T}}(Y, X)= & \int_{0}^{T} \frac{1}{\sqrt{Y_{s}}}\left[\begin{array}{c}
(\widetilde{a}-a)-(\widetilde{b}-b) Y_{s} \\
(\widetilde{\alpha}-\alpha)-(\widetilde{\beta}-\beta) Y_{s}
\end{array}\right]^{\top}\left[\begin{array}{cc}
\sigma_{1} & \sigma_{2} \varrho \\
0 & \sigma_{2} \sqrt{1-\varrho^{2}}
\end{array}\right]^{-1}\left[\begin{array}{l}
\mathrm{d} W_{s} \\
\mathrm{~d} B_{s}
\end{array}\right] \\
& -\frac{1}{2} \int_{0}^{T} \frac{1}{Y_{s}}\left[\begin{array}{c}
(\widetilde{a}-a)-(\widetilde{b}-b) Y_{s} \\
(\widetilde{\alpha}-\alpha)-(\widetilde{\beta}-\beta) Y_{s}
\end{array}\right]^{\top} \boldsymbol{S}^{-1}\left[\begin{array}{c}
(\widetilde{a}-a)-(\widetilde{b}-b) Y_{s} \\
(\widetilde{\alpha}-\alpha)-(\widetilde{\beta}-\beta) Y_{s}
\end{array}\right] \mathrm{d} s .
\end{aligned}
$$

Writing $\boldsymbol{r}=\boldsymbol{r}_{\boldsymbol{\theta}, T}$ and $\boldsymbol{h}=\boldsymbol{h}_{T}$ for the sake of simplicity, we obtain

$$
\log \frac{\mathrm{d} \mathbb{P}_{\boldsymbol{\theta}+\boldsymbol{r h}, T}}{\mathrm{~d} \mathbb{P}_{\boldsymbol{\theta}, T}}(Y, X)=I_{1}-\frac{1}{2} I_{2}
$$

where

$$
\begin{aligned}
& I_{1}:=\int_{0}^{T} \frac{1}{\sqrt{Y_{s}}}\left[\begin{array}{l}
r_{1} h_{1}-r_{3} h_{3} Y_{s} \\
r_{2} h_{2}-r_{4} h_{4} Y_{s}
\end{array}\right]^{\top}\left[\begin{array}{cc}
\sigma_{1} & \sigma_{2} \varrho \\
0 & \sigma_{2} \sqrt{1-\varrho^{2}}
\end{array}\right]^{-1}\left[\begin{array}{l}
\mathrm{d} W_{s} \\
\mathrm{~d} B_{s}
\end{array}\right], \\
& I_{2}:=\int_{0}^{T} \frac{1}{Y_{s}}\left[\begin{array}{l}
r_{1} h_{1}-r_{3} h_{3} Y_{s} \\
r_{2} h_{2}-r_{4} h_{4} Y_{s}
\end{array}\right]^{\top} \boldsymbol{S}^{-1}\left[\begin{array}{l}
r_{1} h_{1}-r_{3} h_{3} Y_{s} \\
r_{2} h_{2}-r_{4} h_{4} Y_{s}
\end{array}\right] \mathrm{d} s .
\end{aligned}
$$


We have

$$
\frac{1}{\sqrt{Y_{s}}}\left[\begin{array}{c}
r_{1} h_{1}-r_{3} h_{3} Y_{s} \\
r_{2} h_{2}-r_{4} h_{4} Y_{s}
\end{array}\right]^{\top}=\frac{1}{\sqrt{Y_{s}}}\left[\begin{array}{l}
h_{1} \\
h_{2}
\end{array}\right]^{\top}\left[\begin{array}{cc}
r_{1} & 0 \\
0 & r_{2}
\end{array}\right]-\sqrt{Y_{s}}\left[\begin{array}{l}
h_{3} \\
h_{4}
\end{array}\right]^{\top}\left[\begin{array}{cc}
r_{3} & 0 \\
0 & r_{4}
\end{array}\right],
$$

hence

$$
\begin{aligned}
I_{1}= & {\left[\begin{array}{l}
h_{1} \\
h_{2}
\end{array}\right]^{\top}\left[\begin{array}{cc}
r_{1} & 0 \\
0 & r_{2}
\end{array}\right]\left[\begin{array}{cc}
\sigma_{1} & \sigma_{2} \varrho \\
0 & \sigma_{2} \sqrt{1-\varrho^{2}}
\end{array}\right]^{-1}\left[\begin{array}{c}
\int_{0}^{T} \frac{\mathrm{d} W_{s}}{\sqrt{Y_{s}}} \\
\int_{0}^{T} \frac{\mathrm{d} B_{s}}{\sqrt{Y_{s}}}
\end{array}\right] } \\
& +\left[\begin{array}{l}
h_{3} \\
h_{4}
\end{array}\right]^{\top}\left[\begin{array}{cc}
r_{3} & 0 \\
0 & r_{4}
\end{array}\right]\left[\begin{array}{cc}
\sigma_{1} & \sigma_{2} \varrho \\
0 & \sigma_{2} \sqrt{1-\varrho^{2}}
\end{array}\right]^{-1}\left[\begin{array}{l}
-\int_{0}^{T} \sqrt{Y_{s}} \mathrm{~d} W_{s} \\
-\int_{0}^{T} \sqrt{Y_{s}} \mathrm{~d} B_{s}
\end{array}\right]=\boldsymbol{\Delta}_{\boldsymbol{\theta}, T}(Y, X),
\end{aligned}
$$

and

$$
\begin{aligned}
I_{2}= & \int_{0}^{T} \frac{\mathrm{d} s}{Y_{s}}\left[\begin{array}{l}
h_{1} \\
h_{2}
\end{array}\right]^{\top}\left[\begin{array}{ll}
r_{1} & 0 \\
0 & r_{2}
\end{array}\right] \boldsymbol{S}^{-1}\left[\begin{array}{ll}
r_{1} & 0 \\
0 & r_{2}
\end{array}\right]\left[\begin{array}{l}
h_{1} \\
h_{2}
\end{array}\right]-T\left[\begin{array}{l}
h_{1} \\
h_{2}
\end{array}\right]^{\top}\left[\begin{array}{cc}
r_{1} & 0 \\
0 & r_{2}
\end{array}\right] \boldsymbol{S}^{-1}\left[\begin{array}{cc}
r_{3} & 0 \\
0 & r_{4}
\end{array}\right]\left[\begin{array}{l}
h_{3} \\
h_{4}
\end{array}\right] \\
& +T\left[\begin{array}{l}
h_{3} \\
h_{4}
\end{array}\right]^{\top}\left[\begin{array}{cc}
r_{3} & 0 \\
0 & r_{4}
\end{array}\right] \boldsymbol{S}^{-1}\left[\begin{array}{cc}
r_{1} & 0 \\
0 & r_{2}
\end{array}\right]\left[\begin{array}{l}
h_{1} \\
h_{2}
\end{array}\right]+\int_{0}^{T} Y_{s} \mathrm{~d} s\left[\begin{array}{l}
h_{3} \\
h_{4}
\end{array}\right]^{\top}\left[\begin{array}{cc}
r_{3} & 0 \\
0 & r_{4}
\end{array}\right] \boldsymbol{S}^{-1}\left[\begin{array}{cc}
r_{3} & 0 \\
0 & r_{4}
\end{array}\right]\left[\begin{array}{l}
h_{3} \\
h_{4}
\end{array}\right] \\
= & \boldsymbol{J}_{\boldsymbol{\theta}, T}(Y, X),
\end{aligned}
$$

hence we conclude the assertion.

\subsubsection{Subcritical case}

2.3.7 Theorem. If $a \in\left(\frac{\sigma_{1}^{2}}{2}, \infty\right), b \in \mathbb{R}_{++}$, and $\alpha, \beta \in \mathbb{R}$, then the family $\left(\mathcal{E}_{T}\right)_{T \in \mathbb{R}_{++}}$ of statistical experiments, given in (2.3.9), is $L A N$ at $\boldsymbol{\theta}:=(a, \alpha, b, \beta)$ with scaling matrices $\boldsymbol{r}_{\boldsymbol{\theta}, T}:=\frac{1}{\sqrt{T}} \boldsymbol{I}_{4}, T \in \mathbb{R}_{++}$, and with information matrix

$$
\boldsymbol{J}_{\boldsymbol{\theta}}:=\left[\begin{array}{cc}
\mathbb{E}\left(\frac{1}{Y_{\infty}}\right) & -1 \\
-1 & \mathbb{E}\left(Y_{\infty}\right)
\end{array}\right] \otimes \boldsymbol{S}^{-1}
$$

Consequently, the family $\left(C\left(\mathbb{R}_{+}, \mathbb{R}^{2}\right), \mathcal{B}\left(C\left(\mathbb{R}_{+}, \mathbb{R}^{2}\right)\right),\left\{\mathbb{P}_{\boldsymbol{\theta}+\boldsymbol{h} / \sqrt{T, T}}: \boldsymbol{h} \in \mathbb{R}^{4}\right\}\right)_{T \in \mathbb{R}_{++}}$of statistical experiments converges to the statistical experiment $\left(\mathbb{R}^{4} \times \mathbb{R}^{4 \times 4}, \mathcal{B}\left(\mathbb{R}^{4} \times \mathbb{R}^{4 \times 4}\right),\left\{\mathcal{N}_{4}\left(\boldsymbol{J}_{\theta} \boldsymbol{h}, \boldsymbol{J}_{\theta}\right)\right.\right.$ : $\left.h \in \mathbb{R}^{4}\right\}$ ) as $T \rightarrow \infty$.

For the proof we need a so-called stable central limit theorem for multidimensional continuous local martingales.

2.3.8 Theorem. (van Zanten [48, Theorem 4.1]) Let $\left(\Omega, \mathcal{F},\left(\mathcal{F}_{t}\right)_{t \in \mathbb{R}_{+}}, \mathbb{P}\right)$ be a filtered probability space satisfying the usual conditions. Let $\left(\boldsymbol{M}_{t}\right)_{t \in \mathbb{R}_{+}}$be a d-dimensional continuous 
local martingale with respect to the filtration $\left(\mathcal{F}_{t}\right)_{t \in \mathbb{R}_{+}}$such that $\mathbb{P}\left(\boldsymbol{M}_{0}=0\right)=1$. Suppose that there exists a function $Q: \mathbb{R}_{+} \rightarrow \mathbb{R}^{d \times d}$ such that $\boldsymbol{Q}(t)$ is an invertible (non-random) matrix for all $t \in \mathbb{R}_{+}, \lim _{t \rightarrow \infty}\|\boldsymbol{Q}(t)\|=0$ and

$$
\boldsymbol{Q}(t)\langle\boldsymbol{M}\rangle_{t} \boldsymbol{Q}(t)^{\top} \stackrel{\mathbb{P}}{\longrightarrow} \boldsymbol{\eta} \boldsymbol{\eta}^{\top} \quad \text { as } t \rightarrow \infty,
$$

where $\boldsymbol{\eta}$ is a $d \times d$ random matrix. Then, for each $\mathbb{R}^{k}$-valued random vector $\boldsymbol{v}$ defined on $(\Omega, \mathcal{F}, \mathbb{P})$, we have

$$
\left(\boldsymbol{Q}(t) \boldsymbol{M}_{t}, \boldsymbol{v}\right) \stackrel{\mathcal{D}}{\longrightarrow}(\boldsymbol{\eta} \boldsymbol{Z}, \boldsymbol{v}) \quad \text { as } t \rightarrow \infty,
$$

where $\boldsymbol{Z}$ is a d-dimensional standard normally distributed random vector independent of $(\boldsymbol{\eta}, \boldsymbol{v})$.

The Theorem 2.3.8 remains true if the function $\boldsymbol{Q}$ is defined only on an interval $\left[t_{0}, \infty\right)$ with some $t_{0} \in \mathbb{R}_{++}$.

Proof of Theorem 2.3.7. By part (i) of Theorem 2.3.4, $\mathbb{E}\left(Y_{\infty}\right)=\frac{a}{b}$ and $\mathbb{E}\left(\frac{1}{Y_{\infty}}\right)=\frac{2 b}{2 a-\sigma_{1}^{2}}$, and hence, part (ii) of Theorem 2.3.4 implies

$$
\frac{1}{T} \int_{0}^{T} Y_{s} \mathrm{~d} s \stackrel{\text { a.s. }}{\longrightarrow} \mathbb{E}\left(Y_{\infty}\right) \quad \text { and } \quad \frac{1}{T} \int_{0}^{T} \frac{\mathrm{d} s}{Y_{s}} \stackrel{\text { a.s. }}{\longrightarrow} \mathbb{E}\left(\frac{1}{Y_{\infty}}\right) \quad \text { as } T \rightarrow \infty .
$$

Thus, using $\boldsymbol{r}_{\boldsymbol{\theta}, T}=\frac{1}{\sqrt{T}}\left(\boldsymbol{I}_{2} \otimes \boldsymbol{I}_{2}\right), T \in \mathbb{R}_{++}$, and applying the identity $(\boldsymbol{A} \otimes \boldsymbol{B})(\boldsymbol{C} \otimes \boldsymbol{D})=$ $(\boldsymbol{A C}) \otimes(\boldsymbol{B D})$, we obtain

$$
\begin{aligned}
\boldsymbol{J}_{\boldsymbol{\theta}, T}(Y, X) & =\left(\boldsymbol{I}_{2} \otimes \boldsymbol{I}_{2}\right)\left(\left[\begin{array}{cc}
\frac{1}{T} \int_{0}^{T} \frac{\mathrm{d} s}{Y_{s}} & -1 \\
-1 & \frac{1}{T} \int_{0}^{T} Y_{s} \mathrm{~d} s
\end{array}\right] \otimes \boldsymbol{S}^{-1}\right)\left(\boldsymbol{I}_{2} \otimes \boldsymbol{I}_{2}\right) \\
& \stackrel{\text { a.s. }}{\longrightarrow}\left[\begin{array}{cc}
\mathbb{E}\left(\frac{1}{Y_{\infty}}\right) & -1 \\
-1 & \mathbb{E}\left(Y_{\infty}\right)
\end{array}\right] \otimes \boldsymbol{S}^{-1}=\boldsymbol{J}_{\boldsymbol{\theta}} \quad \text { as } T \rightarrow \infty .
\end{aligned}
$$

Moreover,

$$
\boldsymbol{M}_{T}:=\left[\begin{array}{c}
\int_{0}^{T} \frac{\mathrm{d} W_{s}}{\sqrt{Y_{s}}} \\
\int_{0}^{T} \frac{\mathrm{d} B_{s}}{\sqrt{Y_{s}}} \\
-\int_{0}^{T} \sqrt{Y_{s}} \mathrm{~d} W_{s} \\
-\int_{0}^{T} \sqrt{Y_{s}} \mathrm{~d} B_{s}
\end{array}\right], \quad T \in \mathbb{R}_{+},
$$

is a 4-dimensional continuous local martingale with quadratic variation process

$$
\langle\boldsymbol{M}\rangle_{T}=\left[\begin{array}{cc}
\int_{0}^{T} \frac{\mathrm{d} s}{Y_{s}} & -T \\
-T & \int_{0}^{T} Y_{s} \mathrm{~d} s
\end{array}\right] \otimes \boldsymbol{I}_{2}, \quad t \in \mathbb{R}_{+} .
$$

By (2.3.10), we have

$$
\frac{1}{T}\langle\boldsymbol{M}\rangle_{T} \stackrel{\text { a.s. }}{\longrightarrow}\left[\begin{array}{cc}
\mathbb{E}\left(\frac{1}{Y_{\infty}}\right) & -1 \\
-1 & \mathbb{E}\left(Y_{\infty}\right)
\end{array}\right] \otimes \boldsymbol{I}_{2} \quad \text { as } T \rightarrow \infty .
$$


Hence, Theorem 2.3 .8 yields

$$
\frac{1}{\sqrt{T}} \boldsymbol{M}_{T} \stackrel{\mathcal{D}}{\longrightarrow} \mathcal{N}_{4}\left(0,\left[\begin{array}{cc}
\mathbb{E}\left(\frac{1}{Y_{\infty}}\right) & -1 \\
-1 & \mathbb{E}\left(Y_{\infty}\right)
\end{array}\right] \otimes \boldsymbol{I}_{2}\right) \quad \text { as } T \rightarrow \infty,
$$

consequently, as $T \rightarrow \infty$, we have

$$
\begin{aligned}
& \boldsymbol{\Delta}_{\boldsymbol{\theta}, T}(Y, X)=\frac{1}{\sqrt{T}}\left(\boldsymbol{I}_{2} \otimes\left[\begin{array}{cc}
\sigma_{1} & \sigma_{2} \varrho \\
0 & \sigma_{2} \sqrt{1-\varrho^{2}}
\end{array}\right]^{-1}\right) \boldsymbol{M}_{T} \\
& \stackrel{\mathcal{D}}{\longrightarrow}\left(\boldsymbol{I}_{2} \otimes\left[\begin{array}{cc}
\sigma_{1} & \sigma_{2} \varrho \\
0 & \sigma_{2} \sqrt{1-\varrho^{2}}
\end{array}\right]^{-1}\right) \mathcal{N}_{4}\left(0,\left[\begin{array}{cc}
\mathbb{E}\left(\frac{1}{Y_{\infty}}\right) & -1 \\
-1 & \mathbb{E}\left(Y_{\infty}\right)
\end{array}\right] \otimes \boldsymbol{I}_{2}\right) \\
& \stackrel{\mathcal{D}}{=} \mathcal{N}_{4}\left(0,\left(\boldsymbol{I}_{2} \otimes\left[\begin{array}{cc}
\sigma_{1} & \sigma_{2} \varrho \\
0 & \sigma_{2} \sqrt{1-\varrho^{2}}
\end{array}\right]^{-1}\right)\left(\left[\begin{array}{cc}
\mathbb{E}\left(\frac{1}{Y_{\infty}}\right) & -1 \\
-1 & \mathbb{E}\left(Y_{\infty}\right)
\end{array}\right] \otimes \boldsymbol{I}_{2}\right)\right. \\
& \times\left(\boldsymbol{I}_{2} \otimes\left[\begin{array}{cc}
\sigma_{1} & \sigma_{2} \varrho \\
0 & \sigma_{2} \sqrt{1-\varrho^{2}}
\end{array}\right]^{-1}\right) \\
& =\mathcal{N}_{4}\left(0,\left[\begin{array}{cc}
\mathbb{E}\left(\frac{1}{Y_{\infty}}\right) & -1 \\
-1 & \mathbb{E}\left(Y_{\infty}\right)
\end{array}\right] \otimes \boldsymbol{S}^{-1}\right)=\mathcal{N}_{4}\left(0, \boldsymbol{J}_{\boldsymbol{\theta}}\right)
\end{aligned}
$$

Thus,

$$
\mathcal{L}\left(\left(\boldsymbol{\Delta}_{\boldsymbol{\theta}, T}, \boldsymbol{J}_{\boldsymbol{\theta}, T}\right) \mid \mathbb{P}_{\boldsymbol{\theta}, T}\right) \Rightarrow \mathcal{N}_{4}\left(0, \boldsymbol{J}_{\boldsymbol{\theta}}\right) \times \delta_{\boldsymbol{J}_{\boldsymbol{\theta}}} \quad \text { as } T \rightarrow \infty,
$$

yielding by Remark 2.1.3, that the family $\left(\mathcal{E}_{T}\right)_{T \in \mathbb{R}_{++}}$of statistical experiments is LAN at $\boldsymbol{\theta}$.

2.3.9 Remark. Applying Theorem 2.2.3 for functions $\psi_{1}(a, \alpha, b, \beta):=a-a_{0}, \psi_{2}(a, \alpha, b, \beta):=$ $\alpha-\alpha_{0}, \psi_{3}(a, \alpha, b, \beta):=b-b_{0}$, and $\psi_{4}(a, \alpha, b, \beta):=\beta-\beta_{0}, \quad(a, \alpha, b, \beta) \in \mathbb{R}_{++} \times \mathbb{R}^{3}$, one can obtain that the family of tests that reject for values

$$
\begin{aligned}
S_{\boldsymbol{\theta}_{0}, T}^{(1)} & :=\frac{\sqrt{2 a_{0}-\sigma_{1}^{2}}}{\sigma_{1}^{2} \sqrt{a_{0} b_{0} T}} \int_{0}^{T} \frac{a_{0}-b_{0} Y_{s}}{Y_{s}}\left[\mathrm{~d} Y_{s}-\left(a_{0}-b_{0} Y_{s}\right) \mathrm{d} s\right] \\
S_{\boldsymbol{\theta}_{0}, T}^{(2)} & :=\frac{\sqrt{2 a_{0}-\sigma_{1}^{2}}}{\sigma_{1} \sigma_{2} \sqrt{a_{0} b_{0} T}} \int_{0}^{T} \frac{a_{0}-b_{0} Y_{s}}{Y_{s}}\left[\mathrm{~d} X_{s}-\left(\alpha_{0}-\beta_{0} Y_{s}\right) \mathrm{d} s\right] \\
S_{\boldsymbol{\theta}_{0}, T}^{(3)} & :=\frac{1}{\sigma_{1}^{2} \sqrt{2 b_{0} T}} \int_{0}^{T} \frac{2 a_{0}-\sigma_{1}^{2}-2 b_{0} Y_{s}}{Y_{s}}\left[\mathrm{~d} Y_{s}-\left(a_{0}-b_{0} Y_{s}\right) \mathrm{d} s\right] \\
S_{\boldsymbol{\theta}_{0}, T}^{(4)} & :=\frac{1}{\sigma_{1} \sigma_{2} \sqrt{2 b_{0} T}} \int_{0}^{T} \frac{2 a_{0}-\sigma_{1}^{2}-2 b_{0} Y_{s}}{Y_{s}}\left[\mathrm{~d} X_{s}-\left(\alpha_{0}-\beta_{0} Y_{s}\right) \mathrm{d} s\right],
\end{aligned}
$$


exceeding $z_{\alpha}$, respectively, are asymptotically optimal for testing $H_{0}^{(1)}: a \leqslant a_{0}$ against $H_{1}^{(1)}: a>a_{0}, H_{0}^{(2)}: \alpha \leqslant \alpha_{0}$ against $H_{1}^{(2)}: \alpha>\alpha_{0}, H_{0}^{(3)}: b \leqslant b_{0}$ against $H_{1}^{(3)}: b>b_{0}$, and $H_{0}^{(4)}: \beta \leqslant \beta_{0}$ against $H_{1}^{(4)}: \beta>\beta_{0}$, respectively, where $\boldsymbol{\theta}_{0}=\left(a_{0}, \alpha_{0}, b_{0}, \beta_{0}\right)$ with $a_{0} \in\left(\frac{\sigma_{1}^{2}}{2}, \infty\right), b_{0} \in \mathbb{R}_{++}, \alpha_{0}, \beta_{0} \in \mathbb{R}$. Indeed,

$$
\boldsymbol{J}_{\boldsymbol{\theta}_{0}}^{-1}=\left[\begin{array}{cc}
\mathbb{E}\left(\frac{1}{Y_{\infty}}\right) & -1 \\
-1 & \mathbb{E}\left(Y_{\infty}\right)
\end{array}\right]^{-1} \otimes \boldsymbol{S}=\frac{2 a_{0}-\sigma_{1}^{2}}{\sigma_{1}^{2}}\left[\begin{array}{cc}
\frac{a_{0}}{b_{0}} & 1 \\
1 & \frac{2 b_{0}}{2 a_{0}-\sigma_{1}^{2}}
\end{array}\right] \otimes \boldsymbol{S},
$$

hence

$$
\begin{aligned}
\boldsymbol{J}_{\boldsymbol{\theta}_{0}}^{-1} \boldsymbol{\Delta}_{\boldsymbol{\theta}_{0}, T} & =\left(\left[\begin{array}{cc}
\mathbb{E}\left(\frac{1}{Y_{\infty}}\right) & -1 \\
-1 & \mathbb{E}\left(Y_{\infty}\right)
\end{array}\right]^{-1} \otimes \boldsymbol{S}\right) \frac{1}{\sqrt{T}}\left(\boldsymbol{I}_{2} \otimes\left[\begin{array}{cc}
\sigma_{1} & \sigma_{2} \varrho \\
0 & \sigma_{2} \sqrt{1-\varrho^{2}}
\end{array}\right]^{-1}\right) \boldsymbol{M}_{T} \\
& =\frac{1}{\sqrt{T}}\left(\left[\begin{array}{cc}
\mathbb{E}\left(\frac{1}{Y_{\infty}}\right) & -1 \\
-1 & \mathbb{E}\left(Y_{\infty}\right)
\end{array}\right]^{-1} \otimes\left[\begin{array}{cc}
\sigma_{1} & 0 \\
\sigma_{2} \varrho & \sigma_{2} \sqrt{1-\varrho^{2}}
\end{array}\right]\right) \int_{0}^{T}\left(\left[\begin{array}{c}
\frac{1}{\sqrt{Y_{s}}} \\
-\sqrt{Y_{s}}
\end{array}\right] \otimes\left[\begin{array}{l}
\mathrm{d} W_{s} \\
\mathrm{~d} B_{s}
\end{array}\right]\right) \\
& =\frac{1}{\sqrt{T}} \int_{0}^{T}\left(\left[\begin{array}{cc}
\mathbb{E}\left(\frac{1}{Y_{\infty}}\right) & -1 \\
-1 & \mathbb{E}\left(Y_{\infty}\right)
\end{array}\right]^{-1}\left[\begin{array}{c}
\frac{1}{\sqrt{Y_{s}}} \\
-\sqrt{Y_{s}}
\end{array}\right]\right) \otimes\left(\left[\begin{array}{cc}
\sigma_{1} & 0 \\
\sigma_{2} \varrho & \sigma_{2} \sqrt{1-\varrho^{2}}
\end{array}\right]\left[\begin{array}{c}
\mathrm{d} W_{s} \\
\mathrm{~d} B_{s}
\end{array}\right]\right) \\
& =\frac{1}{\sqrt{T}} \int_{0}^{T}\left(\left[\begin{array}{cc}
\mathbb{E}\left(\frac{1}{Y_{\infty}}\right) & -1 \\
-1 & \mathbb{E}\left(Y_{\infty}\right)
\end{array}\right]^{-1}\left[\begin{array}{c}
\frac{1}{Y_{s}} \\
-1
\end{array}\right]\right) \otimes\left[\begin{array}{c}
\mathrm{d} Y_{s}-\left(a_{0}-b_{0} Y_{s}\right) \mathrm{d} s \\
\mathrm{~d} X_{s}-\left(\alpha_{0}-\beta_{0} Y_{s}\right) \mathrm{d} s
\end{array}\right] \\
& =\frac{1}{\sqrt{T}} \int_{0}^{T}\left[\begin{array}{c}
\frac{\left(2 a_{0}-\sigma_{1}^{2}\right)\left(a_{0}-b_{0} Y_{s}\right)}{\sigma_{1}^{2} b_{0} Y_{s}} \\
\frac{\left(2 a_{0}-\sigma_{2}^{2}-2 b_{0} Y_{s}\right)}{\sigma_{1}^{2} Y_{s}}
\end{array}\right] \otimes\left[\begin{array}{c}
\mathrm{d} Y_{s}-\left(a_{0}-b_{0} Y_{s}\right) \mathrm{d} s \\
\mathrm{~d} X_{s}-\left(\alpha_{0}-\beta_{0} Y_{s}\right) \mathrm{d} s
\end{array}\right]
\end{aligned}
$$

where we used

$$
\left[\begin{array}{cc}
\sigma_{1} & 0 \\
\sigma_{2} \varrho & \sigma_{2} \sqrt{1-\varrho^{2}}
\end{array}\right]\left[\begin{array}{l}
\mathrm{d} W_{s} \\
\mathrm{~d} B_{s}
\end{array}\right]=\frac{1}{\sqrt{Y_{s}}}\left[\begin{array}{c}
\mathrm{d} Y_{s}-\left(a_{0}-b_{0} Y_{s}\right) \mathrm{d} s \\
\mathrm{~d} X_{s}-\left(\alpha_{0}-\beta_{0} Y_{s}\right) \mathrm{d} s
\end{array}\right]
$$

following from (2.3.6), and $\left(\psi_{i}\right)^{\prime}\left(a_{0}, \alpha_{0}, b_{0}, \beta_{0}\right)=\boldsymbol{e}_{i}, \quad i \in\{1,2,3,4\}$.

\subsubsection{Critical case}

2.3.10 Theorem. If $a \in\left(\frac{\sigma_{1}^{2}}{2}, \infty\right), b=0$, and $\alpha, \beta \in \mathbb{R}$, then the family $\left(\mathcal{E}_{T}\right)_{T \in \mathbb{R}_{++}}$of statistical experiments, given in (2.3.9), is $L A Q$ at $\boldsymbol{\theta}:=(a, \alpha, b, \beta)$ with scaling matrices

$$
\boldsymbol{r}_{\boldsymbol{\theta}, T}:=\left[\begin{array}{cc}
\frac{1}{\sqrt{\log T}} & 0 \\
0 & \frac{1}{T}
\end{array}\right] \otimes \boldsymbol{I}_{2}, \quad T \in \mathbb{R}_{++},
$$

and with

$$
\left(\boldsymbol{\Delta}_{\boldsymbol{\theta}, T}(Y, X), \boldsymbol{J}_{\boldsymbol{\theta}, T}(Y, X)\right) \stackrel{\mathcal{D}}{\longrightarrow}\left(\boldsymbol{\Delta}_{\boldsymbol{\theta}}, \boldsymbol{J}_{\boldsymbol{\theta}}\right) \quad \text { as } T \rightarrow \infty,
$$


where

$$
\boldsymbol{\Delta}_{\boldsymbol{\theta}}:=\left[\begin{array}{c}
\left(a-\frac{\sigma_{1}^{2}}{2}\right)^{-1 / 2}\left[\begin{array}{cc}
\sigma_{1} & \sigma_{2} \varrho \\
0 & \sigma_{2} \sqrt{1-\varrho^{2}}
\end{array}\right]^{-1} \boldsymbol{Z}_{2} \\
\boldsymbol{S}^{-1}\left[\begin{array}{l}
a-\mathcal{Y}_{1} \\
\alpha-\mathcal{X}_{1}
\end{array}\right]
\end{array}\right], \quad \boldsymbol{J}_{\boldsymbol{\theta}}:=\left[\begin{array}{cc}
\left(a-\frac{\sigma_{1}^{2}}{2}\right)^{-1} & 0 \\
0 & \int_{0}^{1} \mathcal{Y}_{s} \mathrm{~d} s
\end{array}\right] \otimes \boldsymbol{S}^{-1},
$$

where $\left(\mathcal{Y}_{t}, \mathcal{X}_{t}\right)_{t \in \mathbb{R}_{+}}$is the unique strong solution of the $S D E$

$$
\left\{\begin{array}{l}
\mathrm{d} \mathcal{Y}_{t}=a \mathrm{~d} t+\sigma_{1} \sqrt{\mathcal{Y}_{t}} \mathrm{~d} \mathcal{W}_{t}, \\
\mathrm{~d} \mathcal{X}_{t}=\alpha \mathrm{d} t+\sigma_{2} \sqrt{\mathcal{Y}_{t}}\left(\varrho \mathrm{d} \mathcal{W}_{t}+\sqrt{1-\varrho^{2}} \mathrm{~d} \mathcal{B}_{t}\right),
\end{array} \quad t \in \mathbb{R}_{+},\right.
$$

with initial value $\left(\mathcal{Y}_{0}, \mathcal{X}_{0}\right)=(0,0)$, where $\left(\mathcal{W}_{t}, \mathcal{B}_{t}\right)_{t \in \mathbb{E}_{+}}$is a 2-dimensional standard Wiener process, $Z_{2}$ is a 2-dimensional standard normally distributed random vector independent of $\left(\mathcal{Y}_{1}, \int_{0}^{1} \mathcal{Y}_{t} \mathrm{~d} t, \mathcal{X}_{1}\right)$, and $\boldsymbol{S}$ is defined in (2.3.7). Consequently, the family $\left(C\left(\mathbb{R}_{+}, \mathbb{R}^{2}\right), \mathcal{B}\left(C\left(\mathbb{R}_{+}, \mathbb{R}^{2}\right)\right),\left\{\mathbb{P}_{\boldsymbol{\theta}+r_{\boldsymbol{\theta}, T} \boldsymbol{h}, T}: \boldsymbol{h} \in \mathbb{R}^{4}\right\}\right)_{T \in \mathbb{R}_{++}}$of statistical experiments converges to the statistical experiment $\left(\mathbb{R}^{4} \times \mathbb{R}^{4 \times 4}, \mathcal{B}\left(\mathbb{R}^{4} \times \mathbb{R}^{4 \times 4}\right),\left\{\mathbb{Q}_{\theta, \boldsymbol{h}}: \boldsymbol{h} \in \mathbb{R}^{4}\right\}\right)$ as $T \rightarrow \infty$, where

$$
\mathbb{Q}_{\boldsymbol{\theta}, \boldsymbol{h}}(B):=\mathbb{E}\left(\exp \left\{\boldsymbol{h}^{\top} \boldsymbol{\Delta}_{\boldsymbol{\theta}}-\frac{1}{2} \boldsymbol{h}^{\top} \boldsymbol{J}_{\boldsymbol{\theta}} \boldsymbol{h}\right\} \mathbb{1}_{B}\left(\boldsymbol{\Delta}_{\boldsymbol{\theta}}, \boldsymbol{J}_{\boldsymbol{\theta}}\right)\right), \quad B \in \mathcal{B}\left(\mathbb{R}^{4} \times \mathbb{R}^{4 \times 4}\right), \quad \boldsymbol{h} \in \mathbb{R}^{4} .
$$

If $b=0$ and $\beta \in \mathbb{R}$ are fixed, then the subfamily

$$
\left(C\left(\mathbb{R}_{+}, \mathbb{R}^{2}\right), \mathcal{B}\left(C\left(\mathbb{R}_{+}, \mathbb{R}^{2}\right)\right),\left\{\mathbb{P}_{\boldsymbol{\theta}, T}: a \in\left(\frac{\sigma_{1}^{2}}{2}, \infty\right), \alpha \in \mathbb{R}\right\}\right)_{T \in \mathbb{R}_{++}}
$$

of statistical experiments is LAN at $(a, \alpha)$ with scaling matrices $\boldsymbol{r}_{\boldsymbol{\theta}, T}^{(1)}:=\frac{1}{\sqrt{\log T}} \boldsymbol{I}_{2}, \quad T \in$ $\mathbb{R}_{++}$, and with information matrix $\boldsymbol{J}_{\boldsymbol{\theta}}^{(1)}:=\left(a-\frac{\sigma_{1}^{2}}{2}\right)^{-1} \boldsymbol{S}^{-1}$. Consequently, the family $\left(C\left(\mathbb{R}_{+}, \mathbb{R}^{2}\right), \mathcal{B}\left(C\left(\mathbb{R}_{+}, \mathbb{R}^{2}\right)\right),\left\{\mathbb{P}_{\boldsymbol{\theta}+\boldsymbol{h} / \sqrt{\log T, T}}: \boldsymbol{h}_{1} \in \mathbb{R}^{2}\right\}\right)_{T \in \mathbb{R}_{++}}$of statistical experiments converges to the statistical experiment $\left(\mathbb{R}^{2} \times \mathbb{R}^{2 \times 2}, \mathcal{B}\left(\mathbb{R}^{2} \times \mathbb{R}^{2 \times 2}\right),\left\{\mathcal{N}_{2}\left(\boldsymbol{J}_{\boldsymbol{\theta}}^{(1)} \boldsymbol{h}_{\boldsymbol{1}}, \boldsymbol{J}_{\boldsymbol{\theta}}^{(1)}\right): \boldsymbol{h}_{1} \in \mathbb{R}^{2}\right\}\right)$ as $T \rightarrow \infty$, where $\boldsymbol{h}:=\left(\boldsymbol{h}_{1}, 0\right)^{\top} \in \mathbb{R}^{4}$. 
Proof. We have

$$
\begin{gathered}
\boldsymbol{\Delta}_{\boldsymbol{\theta}, T}(Y, X)=\left(\left[\begin{array}{cc}
\frac{1}{\sqrt{\log T}} & 0 \\
0 & \frac{1}{T}
\end{array}\right] \otimes \boldsymbol{I}_{2}\right)\left(\boldsymbol{I}_{2} \otimes\left[\begin{array}{cc}
\sigma_{1} & \sigma_{2} \varrho \\
0 & \sigma_{2} \sqrt{1-\varrho^{2}}
\end{array}\right]^{-1}\right)\left[\begin{array}{c}
\int_{0}^{T} \frac{\mathrm{d} W_{s}}{\sqrt{Y_{s}}} \\
\int_{0}^{T} \frac{\mathrm{d} B_{s}}{\sqrt{Y_{s}}} \\
-\int_{0}^{T} \sqrt{Y_{s}} \mathrm{~d} W_{s} \\
-\int_{0}^{T} \sqrt{Y_{s}} \mathrm{~d} B_{s}
\end{array}\right] \\
=\left(\left[\begin{array}{cc}
\left(\frac{1}{\log T} \int_{0}^{T} \frac{\mathrm{d} s}{Y_{s}}\right)^{1 / 2} & \left(\frac{1}{T^{2}} \int_{0}^{T} Y_{s} \mathrm{~d} s\right)^{1 / 2}
\end{array}\right] \otimes\left[\begin{array}{cc}
\int_{0}^{T} \frac{\mathrm{d} W_{s}}{\sqrt{Y_{s}}} \\
0 & \sigma_{2} \varrho \\
0 & \sigma_{2} \sqrt{\left.1-\varrho_{0}^{T} \frac{\mathrm{d} s}{Y_{s}}\right)^{1 / 2}}
\end{array}\right]^{-1}\right)\left[\begin{array}{c}
\int_{0}^{T} \frac{\mathrm{d} B_{s}}{\sqrt{Y_{s}}} \\
\left(\int_{0}^{T} \frac{\mathrm{d} s}{Y_{s}}\right)^{1 / 2} \\
-\frac{\int_{0}^{T} \sqrt{Y_{s}} \mathrm{~d} W_{s}}{\left(\int_{0}^{T} Y_{s} \mathrm{~d} s\right)^{1 / 2}} \\
-\frac{\int_{0}^{T} \sqrt{Y_{s}} \mathrm{~d} B_{s}}{\left(\int_{0}^{T} Y_{s} \mathrm{~d} s\right)^{1 / 2}}
\end{array}\right]
\end{gathered}
$$

and

$$
\begin{aligned}
\boldsymbol{J}_{\boldsymbol{\theta}, T}(Y, X) & =\left(\left[\begin{array}{cc}
\frac{1}{\sqrt{\log T}} & 0 \\
0 & \frac{1}{T}
\end{array}\right] \otimes \boldsymbol{I}_{2}\right)\left(\left[\begin{array}{cc}
\int_{0}^{T} \frac{\mathrm{d} s}{Y_{s}} & -T \\
-T & \int_{0}^{T} Y_{s} \mathrm{~d} s
\end{array}\right] \otimes \boldsymbol{S}^{-1}\right)\left(\left[\begin{array}{cc}
\frac{1}{\sqrt{\log T}} & 0 \\
0 & \frac{1}{T}
\end{array}\right] \otimes \boldsymbol{I}_{2}\right) \\
& =\left[\begin{array}{cc}
\frac{1}{\log T} \int_{0}^{T} \frac{\mathrm{d} s}{Y_{s}} & -\frac{1}{\sqrt{\log T}} \\
-\frac{1}{\sqrt{\log T}} & \frac{1}{T^{2}} \int_{0}^{T} Y_{s} \mathrm{~d} s
\end{array}\right] \otimes \boldsymbol{S}^{-1} .
\end{aligned}
$$

It is known that

$$
\frac{1}{\log T} \int_{0}^{T} \frac{\mathrm{d} s}{Y_{s}} \stackrel{\mathbb{P}}{\longrightarrow}\left(a-\frac{\sigma_{1}^{2}}{2}\right)^{-1} \quad \text { as } T \rightarrow \infty,
$$

see, e.g., Overbeck [36, Lemma 5] or Ben Alaya and Kebaier [8, Proposition 2]. Consequently, (2.3.11) will follow from

$$
\begin{aligned}
& \left(\frac{\int_{0}^{T} \frac{\mathrm{d} W_{s}}{\sqrt{Y_{s}}}}{\left(\int_{0}^{T} \frac{\mathrm{d} s}{Y_{s}}\right)^{1 / 2}}, \frac{\int_{0}^{T} \frac{\mathrm{d} B_{s}}{\sqrt{Y_{s}}}}{\left(\int_{0}^{T} \frac{\mathrm{d} s}{Y_{s}}\right)^{1 / 2}}, \frac{\int_{0}^{T} \sqrt{Y_{s}} \mathrm{~d} W_{s}}{\left(\int_{0}^{T} Y_{s} \mathrm{~d} s\right)^{1 / 2}}, \frac{\int_{0}^{T} \sqrt{Y_{s}} \mathrm{~d} B_{s}}{\left(\int_{0}^{T} Y_{s} \mathrm{~d} s\right)^{1 / 2}}, \frac{Y_{T}}{T}, \frac{1}{T^{2}} \int_{0}^{T} Y_{s} \mathrm{~d} s\right) \\
& \stackrel{\mathcal{D}}{\longrightarrow}\left(Z_{2}, \frac{\mathcal{Y}_{1}-a}{\sigma_{1}\left(\int_{0}^{1} \mathcal{Y}_{s} \mathrm{~d} s\right)^{1 / 2}}, Z_{3}, \mathcal{Y}_{1}, \int_{0}^{1} \mathcal{Y}_{s} \mathrm{~d} s\right)
\end{aligned}
$$

as $T \rightarrow \infty$, where $Z_{3}$ is a standard normally distributed random variable independent of $\left(Z_{2}, \mathcal{Y}_{1}, \int_{0}^{1} \mathcal{Y}_{s} \mathrm{~d} s\right)$. Indeed,

$$
\left(\boldsymbol{\Delta}_{\boldsymbol{\theta}, T}\left(\left(Y_{s}, X_{s}\right)_{s \in[0, T]}\right), \boldsymbol{J}_{\boldsymbol{\theta}, T}\left(\left(Y_{s}, X_{s}\right)_{s \in[0, T]}\right)\right) \stackrel{\mathcal{D}}{\longrightarrow}\left(\widetilde{\boldsymbol{\Delta}}_{\boldsymbol{\theta}}, \boldsymbol{J}_{\boldsymbol{\theta}}\right) \quad \text { as } T \rightarrow \infty,
$$


where, by (2.3.7),

$$
\begin{aligned}
& \widetilde{\boldsymbol{\Delta}}_{\boldsymbol{\theta}}:=\left(\left[\begin{array}{cc}
\left(a-\frac{\sigma_{1}^{2}}{2}\right)^{-1 / 2} & 0 \\
0 & \left(\int_{0}^{1} \mathcal{Y}_{s} \mathrm{~d} s\right)^{1 / 2}
\end{array}\right] \otimes\left[\begin{array}{cc}
\sigma_{1} & \sigma_{2} \varrho \\
0 & \sigma_{2} \sqrt{1-\varrho^{2}}
\end{array}\right]^{-1}\right)\left[\begin{array}{c}
\boldsymbol{Z}_{2} \\
\frac{a-\mathcal{Y}_{1}}{\sigma_{1}\left(\int_{0}^{1} \mathcal{Y}_{s} \mathrm{~d} s\right)^{1 / 2}} \\
-Z_{3}
\end{array}\right]
\end{aligned}
$$

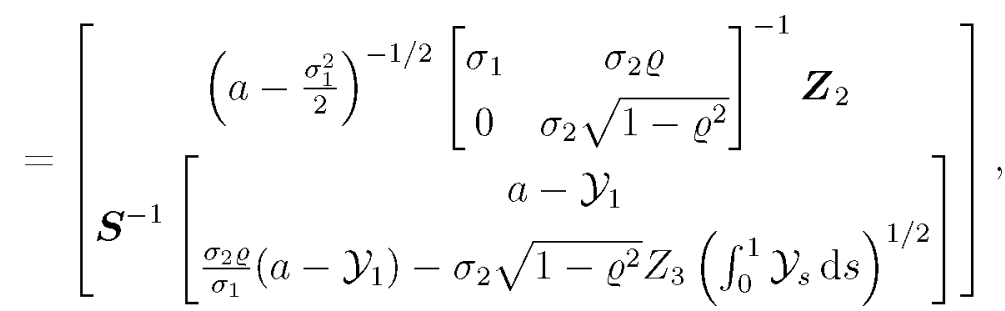

and $\left(\widetilde{\boldsymbol{\Delta}}_{\boldsymbol{\theta}}, \boldsymbol{J}_{\boldsymbol{\theta}}\right) \stackrel{\mathcal{D}}{=}\left(\boldsymbol{\Delta}_{\boldsymbol{\theta}}, \boldsymbol{J}_{\boldsymbol{\theta}}\right)$, since

$$
\left(\mathcal{Y}_{1}, \int_{0}^{1} \mathcal{Y}_{s} \mathrm{~d} s, \frac{\sigma_{2} \varrho}{\sigma_{1}} \frac{\mathcal{Y}_{1}-a}{\int_{0}^{1} \mathcal{Y}_{s} \mathrm{~d} s}+\frac{\sigma_{2} \sqrt{1-\varrho^{2}}}{\left(\int_{0}^{1} \mathcal{Y}_{s} \mathrm{~d} s\right)^{1 / 2}} Z_{3}\right) \stackrel{\mathcal{D}}{=}\left(\mathcal{Y}_{1}, \int_{0}^{1} \mathcal{Y}_{s} \mathrm{~d} s, \frac{\mathcal{X}_{1}-\alpha}{\int_{0}^{1} \mathcal{Y}_{s} \mathrm{~d} s}\right),
$$

and $Z_{2}$ is independent of $\left(Z_{3}, \mathcal{Y}_{1}, \int_{0}^{1} \mathcal{Y}_{s} \mathrm{~d} s\right)$ and of $\left(\mathcal{Y}_{1}, \int_{0}^{1} \mathcal{Y}_{s} \mathrm{~d} s, \mathcal{X}_{1}\right)$, see Barczy and Pap [7, Equation (6.9)].

We prove (2.3.14) using continuity theorem. We have

$$
\sigma_{1} \int_{0}^{T} \frac{\mathrm{d} W_{s}}{\sqrt{Y_{s}}}=\log Y_{T}-\log y_{0}+\left(\frac{\sigma_{1}^{2}}{2}-a\right) \int_{0}^{T} \frac{\mathrm{d} s}{Y_{s}}, \quad T \in \mathbb{R}_{+},
$$

see Barczy and Pap [7, Formula (6.16)]. By (2.3.1) and by the assumption $b=0$, one can obtain

$$
\sigma_{1} \int_{0}^{T} \sqrt{Y_{s}} \mathrm{~d} W_{s}=Y_{T}-y_{0}-a T, \quad T \in \mathbb{R}_{+} .
$$

Consequently, $\int_{0}^{T} \frac{\mathrm{d} W_{s}}{\sqrt{Y_{s}}}$ and $\int_{0}^{T} \sqrt{Y_{s}} \mathrm{~d} W_{s}$ are measurable with respect to the $\sigma$-algebra $\sigma\left(Y_{s}, s \in\right.$ 
$[0, T])$. For all $\left(u_{1}, u_{2}, u_{3}, u_{4}, v_{1}, v_{2}\right) \in \mathbb{R}^{6}$ and $T \in \mathbb{R}_{++}$, we have

$$
\begin{aligned}
& \mathbb{E}\left(\operatorname { e x p } \left\{\mathrm{i} u_{1} \frac{\int_{0}^{T} \frac{\mathrm{d} W_{s}}{\sqrt{Y_{s}}}}{\left(\int_{0}^{T} \frac{\mathrm{d} s}{Y_{s}}\right)^{1 / 2}}+\mathrm{i} u_{2} \frac{\int_{0}^{T} \frac{\mathrm{d} B_{s}}{\sqrt{Y_{s}}}}{\left(\int_{0}^{T} \frac{\mathrm{d} s}{Y_{s}}\right)^{1 / 2}}+\mathrm{i} u_{3} \frac{\int_{0}^{T} \sqrt{Y_{s}} \mathrm{~d} W_{s}}{\left(\int_{0}^{T} Y_{s} \mathrm{~d} s\right)^{1 / 2}}+\mathrm{i} u_{4} \frac{\int_{0}^{T} \sqrt{Y_{s}} \mathrm{~d} B_{s}}{\left(\int_{0}^{T} Y_{s} \mathrm{~d} s\right)^{1 / 2}}\right.\right. \\
& \left.\left.+\mathrm{i} v_{1} \frac{1}{T} Y_{T}+\mathrm{i} v_{2} \frac{1}{T^{2}} \int_{0}^{T} Y_{s} \mathrm{~d} s\right\} \mid Y_{s}, s \in[0, T]\right) \\
& =\exp \left\{\mathrm{i} u_{1} \frac{\int_{0}^{T} \frac{\mathrm{d} W_{s}}{\sqrt{Y_{s}}}}{\left(\int_{0}^{T} \frac{\mathrm{d} s}{Y_{s}}\right)^{1 / 2}}+\mathrm{i} u_{3} \frac{\int_{0}^{T} \sqrt{Y_{s}} \mathrm{~d} W_{s}}{\left(\int_{0}^{T} Y_{s} \mathrm{~d} s\right)^{1 / 2}}+\mathrm{i} v_{1} \frac{1}{T} Y_{T}+\mathrm{i} v_{2} \frac{1}{T^{2}} \int_{0}^{T} Y_{s} \mathrm{~d} s\right\} \\
& =\exp \left\{\mathrm{i} u_{1} \frac{\int_{0}^{T} \frac{\mathrm{d} W_{s}}{\sqrt{Y_{s}}}}{\left(\int_{0}^{T} \frac{\mathrm{d} s}{Y_{s}}\right)^{1 / 2}}+\mathrm{i} u_{3} \frac{\int_{0}^{T} \sqrt{Y_{s}} \mathrm{~d} W_{s}}{\left(\int_{0}^{T} Y_{s} \mathrm{~d} s\right)^{1 / 2}}+\mathrm{i} v_{1} \frac{1}{T} Y_{T}+\mathrm{i} v_{2} \frac{1}{T^{2}} \int_{0}^{T} Y_{s} \mathrm{~d} s\right\} \\
& \left.\quad \times \exp \left\{-\frac{1}{2} \int_{0}^{T}\left(\frac{u_{2}}{\int_{0}^{T}} \frac{1}{\int_{0}^{T}} \frac{1}{Y_{t}}\right)^{1 / 2} \frac{1}{Y_{s}}+\frac{u_{4}^{2}}{\int_{0}^{T} Y_{t} \mathrm{~d} t} Y_{s}+\frac{2 u_{2} u_{4}}{\left(\int_{0}^{T} \frac{\mathrm{d} t}{Y_{t}} \int_{0}^{T} Y_{t} \mathrm{~d} t\right)^{1 / 2}}\right) \mathrm{d} s\right\} \\
& =\exp \left\{\mathrm{i} u_{1} \frac{\int_{0}^{T} \frac{\mathrm{d} W_{s}}{\sqrt{Y_{s}}}}{\left(\int_{0}^{T} \frac{\mathrm{d} s}{Y_{s}}\right)^{1 / 2}}+\mathrm{i} u_{3} \frac{\int_{0}^{T} \sqrt{Y_{s}} \mathrm{~d} W_{s}}{\left(\int_{0}^{T} Y_{s} \mathrm{~d} s\right)^{1 / 2}}+\mathrm{i} v_{1} \frac{1}{T} Y_{T}+\mathrm{i} v_{2} \frac{1}{T^{2}} \int_{0}^{T} Y_{s} \mathrm{~d} s\right\} \\
& \quad \times \exp \left\{-\frac{1}{2}\left(u_{2}^{2}+u_{4}^{2}\right)-\frac{T u_{2} u_{4}}{\left(\int_{0}^{T} \frac{\mathrm{d} t}{Y_{t}} \int_{0}^{T} Y_{t} \mathrm{~d} t\right)^{1 / 2}}\right\},
\end{aligned}
$$

where we used the independence of $Y$ and $B$. Consequently, the joint characteristic function of the random vector on the left hand side of (2.3.14) takes the form

$$
\begin{aligned}
& \mathbb{E}\left(\operatorname { e x p } \left\{\mathrm{i} u_{1} \frac{\int_{0}^{T} \frac{\mathrm{d} W_{s}}{\sqrt{Y_{s}}}}{\left(\int_{0}^{T} \frac{\mathrm{d} s}{Y_{s}}\right)^{1 / 2}}+\mathrm{i} u_{2} \frac{\int_{0}^{T} \frac{\mathrm{d} B_{s}}{\sqrt{Y_{s}}}}{\left(\int_{0}^{T} \frac{\mathrm{d} s}{Y_{s}}\right)^{1 / 2}}+\mathrm{i} u_{3} \frac{\int_{0}^{T} \sqrt{Y_{s}} \mathrm{~d} W_{s}}{\left(\int_{0}^{T} Y_{s} \mathrm{~d} s\right)^{1 / 2}}+\mathrm{i} u_{4} \frac{\int_{0}^{T} \sqrt{Y_{s}} \mathrm{~d} B_{s}}{\left(\int_{0}^{T} Y_{s} \mathrm{~d} s\right)^{1 / 2}}\right.\right. \\
& \left.\left.+\mathrm{i} v_{1} \frac{1}{T} Y_{T}+\mathrm{i} v_{2} \frac{1}{T^{2}} \int_{0}^{T} Y_{s} \mathrm{~d} s\right\}\right) \\
& =\mathrm{e}^{-\left(u_{2}^{2}+u_{4}^{2}\right) / 2} \mathbb{E}\left(\exp \left\{\xi_{T}\left(u_{1}, u_{3}, v_{1}, v_{2}\right)-\frac{T u_{2} u_{4}}{\left(\int_{0}^{T} \frac{\mathrm{d} t}{Y_{t}} \int_{0}^{T} Y_{t} \mathrm{~d} t\right)^{1 / 2}}\right\}\right),
\end{aligned}
$$

where

$$
\xi_{T}\left(u_{1}, u_{3}, v_{1}, v_{2}\right):=\mathrm{i} u_{1} \frac{\int_{0}^{T} \frac{\mathrm{d} W_{s}}{\sqrt{Y_{s}}}}{\left(\int_{0}^{T} \frac{\mathrm{d} s}{Y_{s}}\right)^{1 / 2}}+\mathrm{i} u_{3} \frac{\int_{0}^{T} \sqrt{Y_{s}} \mathrm{~d} W_{s}}{\left(\int_{0}^{T} Y_{s} \mathrm{~d} s\right)^{1 / 2}}+\mathrm{i} v_{1} \frac{1}{T} Y_{T}+\mathrm{i} v_{2} \frac{1}{T^{2}} \int_{0}^{T} Y_{s} \mathrm{~d} s .
$$


Ben Alaya and Kebaier [9, proof of Theorem 6] proved

$$
\left(\frac{\log Y_{T}-\log y_{0}+\left(\frac{\sigma_{1}^{2}}{2}-a\right) \int_{0}^{T} \frac{\mathrm{d} s}{Y_{s}}}{\sqrt{\log T}}, \frac{Y_{T}}{T}, \frac{1}{T^{2}} \int_{0}^{T} Y_{s} \mathrm{~d} s\right) \stackrel{\mathcal{D}}{\longrightarrow}\left(\frac{\sigma_{1}}{\sqrt{a-\frac{\sigma_{1}^{2}}{2}}} Z_{1}, \mathcal{Y}_{1}, \int_{0}^{1} \mathcal{Y}_{s} \mathrm{~d} s\right)
$$

as $T \rightarrow \infty$, where $Z_{1}$ is a 1-dimensional standard normally distributed random variable independent of $\left(\mathcal{Y}_{1}, \int_{0}^{1} \mathcal{Y}_{t} \mathrm{~d} t\right)$. Using (2.3.15) we have

$$
\frac{\int_{0}^{T} \frac{\mathrm{d} W_{s}}{\sqrt{Y_{s}}}}{\left(\int_{0}^{T} \frac{\mathrm{d} s}{Y_{s}}\right)^{1 / 2}}=\frac{\frac{1}{\sqrt{\log T}} \frac{1}{\sigma_{1}}\left(\log Y_{T}-\log y_{0}+\left(\frac{\sigma_{1}^{2}}{2}-a\right) \int_{0}^{T} \frac{\mathrm{d} s}{Y_{s}}\right)}{\left(\frac{1}{\log T} \int_{0}^{T} \frac{\mathrm{d} s}{Y_{s}}\right)^{1 / 2}}, \quad T \in \mathbb{R}_{++}
$$

and, by (2.3.13), we conclude

$$
\begin{aligned}
& \left(\frac{\int_{0}^{T} \frac{\mathrm{d} W_{s}}{\sqrt{Y_{s}}}}{\left(\int_{0}^{T} \frac{\mathrm{d} s}{Y_{s}}\right)^{1 / 2}}, \frac{\int_{0}^{T} \sqrt{Y_{s}} \mathrm{~d} W_{s}}{\left(\int_{0}^{T} Y_{s} \mathrm{~d} s\right)^{1 / 2}}, \frac{Y_{T}}{T}, \frac{1}{T^{2}} \int_{0}^{T} Y_{s} \mathrm{~d} s\right) \\
& \stackrel{\mathcal{D}}{\longrightarrow}\left(Z_{1}, \frac{\mathcal{Y}_{1}-a}{\sigma_{1}\left(\int_{0}^{1} \mathcal{Y}_{s} \mathrm{~d} s\right)^{1 / 2}}, \mathcal{Y}_{1}, \int_{0}^{1} \mathcal{Y}_{s} \mathrm{~d} s\right) \text { as } T \rightarrow \infty,
\end{aligned}
$$

thus we derived joint convergence of four coordinates of the left hand side of (2.3.14). Hence

$$
\begin{aligned}
& \mathbb{E}\left(\exp \left\{\xi_{T}\left(u_{1}, u_{3}, v_{1}, v_{2}\right)\right\}\right) \\
& \rightarrow \mathbb{E}\left(\exp \left\{\mathrm{i} u_{1} Z_{1}+\mathrm{i} u_{3} \frac{\mathcal{Y}_{1}-a}{\sigma_{1}\left(\int_{0}^{1} \mathcal{Y}_{s} \mathrm{~d} s\right)^{1 / 2}}+\mathrm{i} v_{1} \mathcal{Y}_{1}+\mathrm{i} v_{2} \int_{0}^{1} \mathcal{Y}_{s} \mathrm{~d} s\right\}\right)
\end{aligned}
$$

as $T \rightarrow \infty$ for all $\left(u_{1}, u_{3}, v_{1}, v_{2}\right) \in \mathbb{R}^{4}$. Using $\left|\exp \left\{\xi_{T}\left(u_{1}, u_{3}, v_{1}, v_{2}\right)\right\}\right|=1$, we have

$$
\begin{aligned}
& \left|\mathbb{E}\left(\exp \left\{\xi_{T}\left(u_{1}, u_{3}, v_{1}, v_{2}\right)-\frac{T u_{2} u_{4}}{\left(\int_{0}^{T} \frac{\mathrm{d} t}{Y_{t}} \int_{0}^{T} Y_{t} \mathrm{~d} t\right)^{1 / 2}}\right\}\right)-\mathbb{E}\left(\exp \left\{\xi_{T}\left(u_{1}, u_{3}, v_{1}, v_{2}\right)\right\}\right)\right| \\
& \quad \leqslant \mathbb{E}\left(\left|\exp \left\{\xi_{T}\left(u_{1}, u_{3}, v_{1}, v_{2}\right)\right\}\right| \exp \left\{-\frac{T u_{2} u_{4}}{\left(\int_{0}^{T} \frac{\mathrm{d} t}{Y_{t}} \int_{0}^{T} Y_{t} \mathrm{~d} t\right)^{1 / 2}}\right\}-1 \mid\right) \\
& \quad=\mathbb{E}\left(\left|\exp \left\{-\frac{T u_{2} u_{4}}{\left(\int_{0}^{T} \frac{\mathrm{d} t}{Y_{t}} \int_{0}^{T} Y_{t} \mathrm{~d} t\right)^{1 / 2}}\right\}-1\right|\right) \rightarrow 0 \quad \text { as } T \rightarrow \infty,
\end{aligned}
$$

by the moment convergence theorem (see, e.g., Stroock [45, Lemma 2.2.1]). Indeed, by (2.3.13), (2.3.16), continuous mapping theorem and Slutsky's lemma,

$$
\left|\exp \left\{-\frac{T u_{2} u_{4}}{\left(\int_{0}^{T} \frac{\mathrm{d} t}{Y_{t}} \int_{0}^{T} Y_{t} \mathrm{~d} t\right)^{1 / 2}}\right\}-1\right|=\left|\exp \left\{-\frac{u_{2} u_{4}}{\sqrt{\log T}\left(\frac{1}{\log T} \int_{0}^{T} \frac{\mathrm{d} t}{Y_{t}} \cdot \frac{1}{T^{2}} \int_{0}^{T} Y_{t} \mathrm{~d} t\right)^{1 / 2}}\right\}-1\right| \stackrel{\mathbb{P}}{\longrightarrow} 0
$$


as $T \rightarrow \infty$, and the family

$$
\left\{\left|\exp \left\{-\frac{T u_{2} u_{4}}{\left(\int_{0}^{T} \frac{\mathrm{d} t}{Y_{t}} \int_{0}^{T} Y_{t} \mathrm{~d} t\right)^{1 / 2}}\right\}-1\right|, T \in \mathbb{R}_{++}\right\}
$$

is uniformly integrable, since, by Cauchy-Schwarz inequality,

$$
\left|\exp \left\{-\frac{T u_{2} u_{4}}{\left(\int_{0}^{T} \frac{\mathrm{d} t}{Y_{t}} \int_{0}^{T} Y_{t} \mathrm{~d} t\right)^{1 / 2}}\right\}-1\right| \leqslant \exp \left\{\frac{T\left|u_{2} u_{4}\right|}{\left(\int_{0}^{T} \frac{\mathrm{d} t}{Y_{t}} \int_{0}^{T} Y_{t} \mathrm{~d} t\right)^{1 / 2}}\right\}+1 \leqslant \exp \left\{\left|u_{2} u_{4}\right|\right\}+1
$$

for all $T \in \mathbb{R}_{++}$. Using (2.3.17), one can conclude

$$
\begin{aligned}
& \mathbb{E}\left(\operatorname { e x p } \left\{\mathrm{i} u_{1} \frac{\int_{0}^{T} \frac{\mathrm{d} W_{s}}{\sqrt{Y_{s}}}}{\left(\int_{0}^{T} \frac{\mathrm{d} s}{Y_{s}}\right)^{1 / 2}}+\mathrm{i} u_{2} \frac{\int_{0}^{T} \frac{\mathrm{d} B_{s}}{\sqrt{Y_{s}}}}{\left(\int_{0}^{T} \frac{\mathrm{d} s}{Y_{s}}\right)^{1 / 2}}+\mathrm{i} u_{3} \frac{\int_{0}^{T} \sqrt{Y_{s}} \mathrm{~d} W_{s}}{\left(\int_{0}^{T} Y_{s} \mathrm{~d} s\right)^{1 / 2}}+\mathrm{i} u_{4} \frac{\int_{0}^{T} \sqrt{Y_{s}} \mathrm{~d} B_{s}}{\left(\int_{0}^{T} Y_{s} \mathrm{~d} s\right)^{1 / 2}}\right.\right. \\
& \left.\left.+\mathrm{i} v_{1} \frac{1}{T} Y_{T}+\mathrm{i} v_{2} \frac{1}{T^{2}} \int_{0}^{T} Y_{s} \mathrm{~d} s\right\}\right) \\
& \rightarrow \mathrm{e}^{-\left(u_{2}^{2}+u_{4}^{2}\right) / 2} \mathbb{E}\left(\exp \left\{\mathrm{i} u_{1} Z_{1}+\mathrm{i} u_{3} \frac{\mathcal{Y}_{1}-a}{\sigma_{1}\left(\int_{0}^{1} \mathcal{Y}_{s} \mathrm{~d} s\right)^{1 / 2}}+\mathrm{i} v_{1} \mathcal{Y}_{1}+\mathrm{i} v_{2} \int_{0}^{1} \mathcal{Y}_{s} \mathrm{~d} s\right\}\right)
\end{aligned}
$$

as $T \rightarrow \infty$. Note that, since $Z_{1}$ is independent of $\left(\mathcal{Y}_{1}, \int_{0}^{1} \mathcal{Y}_{s} \mathrm{~d} s\right)$, we have

$$
\begin{aligned}
& \mathrm{e}^{-\left(u_{2}^{2}+u_{4}^{2}\right) / 2} \mathbb{E}\left(\exp \left\{\mathrm{i} u_{1} Z_{1}+\mathrm{i} u_{3} \frac{\mathcal{Y}_{1}-a}{\sigma_{1}\left(\int_{0}^{1} \mathcal{Y}_{s} \mathrm{~d} s\right)^{1 / 2}}+\mathrm{i} v_{1} \mathcal{Y}_{1}+\mathrm{i} v_{2} \int_{0}^{1} \mathcal{Y}_{s} \mathrm{~d} s\right\}\right) \\
& =\mathbb{E}\left(\mathrm{e}^{\mathrm{i} u_{1} Z_{1}}\right) \mathbb{E}\left(\mathrm{e}^{\mathrm{i} u_{2} Z_{2}}\right) \mathbb{E}\left(\mathrm{e}^{\mathrm{i} u_{3} Z_{3}}\right) \mathbb{E}\left(\exp \left\{\mathrm{i} u_{3} \frac{\mathcal{Y}_{1}-a}{\sigma_{1}\left(\int_{0}^{1} \mathcal{Y}_{s} \mathrm{~d} s\right)^{1 / 2}}+\mathrm{i} v_{1} \mathcal{Y}_{1}+\mathrm{i} v_{2} \int_{0}^{1} \mathcal{Y}_{s} \mathrm{~d} s\right\}\right),
\end{aligned}
$$

where $\left(Z_{2}, Z_{3}\right)$ is a 2-dimensional standard normally distributed random vector, independent of $\left(Z_{1}, \mathcal{Y}_{1}, \int_{0}^{1} \mathcal{Y}_{s} \mathrm{~d} s\right)$, thus one can obtain $(2.3 .14)$ with $Z_{2}:=\left(Z_{1}, Z_{2}\right)$, and hence (2.3.11), which yields $(2.1 .2)$.

It is known that $\mathbb{P}\left(\int_{0}^{1} \mathcal{Y}_{s} \mathrm{~d} s \in \mathbb{R}_{++}\right)=1$ (which has been shown in the proof of Theorem 3.1 in Barczy et al. [5]), hence (2.1.3) holds. Finally, (2.1.4) will follow from

$$
\mathbb{E}\left(\exp \left\{\boldsymbol{h}^{\top} \boldsymbol{\Delta}_{\theta}-\frac{1}{2} \boldsymbol{h}^{\top} \boldsymbol{J}_{\boldsymbol{\theta}} \boldsymbol{h}\right\}\right)=1
$$

for all $\boldsymbol{h} \in \mathbb{R}^{4}$. Writing $\boldsymbol{h}=\left(\boldsymbol{h}_{1}, \boldsymbol{h}_{2}\right)^{\top}, \boldsymbol{h}_{1}, \boldsymbol{h}_{2} \in \mathbb{R}^{2}$, and using the independence of $\boldsymbol{Z}_{2}$ and $\left(\mathcal{Y}_{1}, \int_{0}^{1} \mathcal{Y}_{t} \mathrm{~d} t, \mathcal{X}_{1}\right)$, we have

$$
\mathbb{E}\left(\exp \left\{\boldsymbol{h}^{\top} \boldsymbol{\Delta}_{\boldsymbol{\theta}}-\frac{1}{2} \boldsymbol{h}^{\top} \boldsymbol{J}_{\boldsymbol{\theta}} \boldsymbol{h}\right\}\right)=E_{1} E_{2}
$$


where

$$
\begin{aligned}
& E_{1}:=\mathbb{E}\left(\exp \left\{\left(a-\frac{\sigma_{1}^{2}}{2}\right)^{-1 / 2} \boldsymbol{h}_{1}^{\top}\left[\begin{array}{cc}
\sigma_{1} & \sigma_{2} \varrho \\
0 & \sigma_{2} \sqrt{1-\varrho^{2}}
\end{array}\right]^{-1} \boldsymbol{Z}_{2}-\frac{1}{2}\left(a-\frac{\sigma_{1}^{2}}{2}\right)^{-1} \boldsymbol{h}_{1}^{\top} \boldsymbol{S}^{-1} \boldsymbol{h}_{1}\right\}\right), \\
& E_{2}:=\mathbb{E}\left(\exp \left\{\boldsymbol{h}_{2}^{\top} \boldsymbol{S}^{-1}\left[\begin{array}{l}
a-\mathcal{Y}_{1} \\
\alpha-\mathcal{X}_{1}
\end{array}\right]-\frac{1}{2}\left(\int_{0}^{1} \mathcal{Y}_{s} \mathrm{~d} s\right) \boldsymbol{h}_{2}^{\top} \boldsymbol{S}^{-1} \boldsymbol{h}_{2}\right\}\right) .
\end{aligned}
$$

The moment generating function of the 2-dimensional standard normally distributed random vector $Z_{2}$ has the form

$$
\mathbb{E}\left(\mathrm{e}^{\boldsymbol{v}^{\top} \boldsymbol{Z}_{2}}\right)=\mathrm{e}^{\|\boldsymbol{v}\|^{2} / 2}, \quad \boldsymbol{v} \in \mathbb{R}^{2}
$$

since

$$
\mathbb{E}\left(\mathrm{e}^{\boldsymbol{v}^{\top} \boldsymbol{Z}_{2}}\right)=\frac{1}{2 \pi} \int_{\mathbb{R}^{2}} \mathrm{e}^{\boldsymbol{v}^{\top} \boldsymbol{Z}_{2}-\|\boldsymbol{x}\|^{2} / 2} \mathrm{~d} \boldsymbol{x}=\frac{1}{2 \pi} \int_{\mathbb{R}^{2}} \mathrm{e}^{-\|\boldsymbol{x}-\boldsymbol{v}\|^{2} / 2+\|\boldsymbol{v}\|^{2} / 2} \mathrm{~d} \boldsymbol{x}=\mathrm{e}^{\|\boldsymbol{v}\|^{2} / 2} .
$$

Applying this with

$$
\boldsymbol{v}^{\top}=\left(a-\frac{\sigma_{1}^{2}}{2}\right)^{-1 / 2} \boldsymbol{h}_{1}^{\top}\left[\begin{array}{cc}
\sigma_{1} & \sigma_{2} \varrho \\
0 & \sigma_{2} \sqrt{1-\varrho^{2}}
\end{array}\right]^{-1}, \quad\|\boldsymbol{v}\|^{2}=\boldsymbol{v}^{\top} \boldsymbol{v}=\left(a-\frac{\sigma_{1}^{2}}{2}\right)^{-1} \boldsymbol{h}_{1}^{\top} \boldsymbol{S}^{-1} \boldsymbol{h}_{1}
$$

we obtain $E_{1}=1$. Using Corollary 2.3 .6 for the process $\left(\mathcal{Y}_{t}, \mathcal{X}_{t}\right)_{t \in \mathbb{R}_{+}}$with

$$
\boldsymbol{r}_{\boldsymbol{\theta}, T}=\boldsymbol{r}:=\left[\begin{array}{ll}
0 & 0 \\
0 & 1
\end{array}\right] \otimes \boldsymbol{I}_{2}, \quad \boldsymbol{h}_{T}=\boldsymbol{h}
$$

we obtain

$$
\log \frac{\mathrm{d} \mathbb{P}_{\boldsymbol{\theta}+\boldsymbol{r} \boldsymbol{h}, T}}{\mathrm{~d} \mathbb{P}_{\boldsymbol{\theta}, T}}(\mathcal{Y}, \mathcal{X})=\boldsymbol{h}^{\top} \boldsymbol{\Delta}_{\boldsymbol{\theta}, T}(\mathcal{Y}, \mathcal{X})-\frac{1}{2} \boldsymbol{h}^{\top} \boldsymbol{J}_{\boldsymbol{\theta}, T}(\mathcal{Y}, \mathcal{X}) \boldsymbol{h}
$$

where

$$
\begin{gathered}
\boldsymbol{h}^{\top} \boldsymbol{\Delta}_{\boldsymbol{\theta}, T}(\mathcal{Y}, \mathcal{X})=\boldsymbol{h}^{\top}\left(\left[\begin{array}{ll}
0 & 0 \\
0 & 1
\end{array}\right] \otimes \boldsymbol{I}_{2}\right)\left(\boldsymbol{I}_{2} \otimes\left[\begin{array}{cc}
\sigma_{1} & \sigma_{2} \varrho \\
0 & \sigma_{2} \sqrt{1-\varrho^{2}}
\end{array}\right]^{-1}\right)\left[\begin{array}{c}
\int_{0}^{T} \frac{\mathrm{d} \mathcal{W}_{s}}{\sqrt{\mathcal{Y}_{s}}} \\
\int_{0}^{T} \frac{\mathrm{d} \mathcal{B}_{s}}{\sqrt{\mathcal{Y}_{s}}} \\
-\int_{0}^{T} \sqrt{\mathcal{Y}_{s}} \mathrm{~d} \mathcal{W}_{s} \\
-\int_{0}^{T} \sqrt{\mathcal{Y}_{s}} \mathrm{~d} \mathcal{B}_{s}
\end{array}\right] \\
=-\boldsymbol{h}_{2}^{\top}\left[\begin{array}{ll}
\sigma_{1} & \sigma_{2} \varrho \\
0 & \sigma_{2} \sqrt{1-\varrho^{2}}
\end{array}\right]^{-1}\left[\begin{array}{l}
\int_{0}^{T} \sqrt{\mathcal{Y}_{s}} \mathrm{~d} \mathcal{W}_{s} \\
\int_{0}^{T} \sqrt{\mathcal{Y}_{s}} \mathrm{~d} \mathcal{B}_{s}
\end{array}\right]=-\boldsymbol{h}_{2}^{\top} \boldsymbol{S}^{-1}\left[\begin{array}{cc}
\sigma_{1} & 0 \\
\sigma_{2} \varrho & \sigma_{2} \sqrt{1-\varrho^{2}}
\end{array}\right]\left[\begin{array}{l}
\int_{0}^{T} \sqrt{\mathcal{Y}_{s}} \mathrm{~d} \mathcal{W}_{s} \\
\int_{0}^{T} \sqrt{\mathcal{Y}_{s}} \mathrm{~d} \mathcal{B}_{s}
\end{array}\right] \\
=\boldsymbol{h}_{2}^{\top} \boldsymbol{S}^{-1}\left[\begin{array}{c}
a T-\mathcal{Y}_{T} \\
\alpha T-\mathcal{X}_{T}
\end{array}\right]
\end{gathered}
$$


and

$$
\begin{aligned}
\boldsymbol{h}^{\top} \boldsymbol{J}_{\boldsymbol{\theta}, T}(\mathcal{Y}, \mathcal{X}) \boldsymbol{h} & =\boldsymbol{h}^{\top}\left(\left[\begin{array}{ll}
0 & 0 \\
0 & 1
\end{array}\right] \otimes \boldsymbol{I}_{2}\right)\left(\left[\begin{array}{cc}
\int_{0}^{T} \frac{\mathrm{d} s}{\mathcal{Y}_{s}} & -T \\
-T & \int_{0}^{T} \mathcal{Y}_{s} \mathrm{~d} s
\end{array}\right] \otimes \boldsymbol{S}^{-1}\right)\left(\left[\begin{array}{ll}
0 & 0 \\
0 & 1
\end{array}\right] \otimes \boldsymbol{I}_{2}\right) \boldsymbol{h} \\
& =\left(\int_{0}^{T} \mathcal{Y}_{s} \mathrm{~d} s\right) \boldsymbol{h}_{2}^{\top} \boldsymbol{S}^{-1} \boldsymbol{h}_{2} .
\end{aligned}
$$

By Lemma 2.3.5, the process

$$
\left(\frac{\mathrm{d} \mathbb{P}_{\boldsymbol{\theta}+\boldsymbol{r h}, T}}{\mathrm{~d} \mathbb{P}_{\boldsymbol{\theta}, T}}(\mathcal{Y}, \mathcal{X})\right)_{T \in \mathbb{R}_{+}}=\left(\exp \left\{\boldsymbol{h}_{2}^{\top} \boldsymbol{S}^{-1}\left[\begin{array}{l}
a T-\mathcal{Y}_{T} \\
\alpha T-\mathcal{X}_{T}
\end{array}\right]-\frac{1}{2}\left(\int_{0}^{T} \mathcal{Y}_{s} \mathrm{~d} s\right) \boldsymbol{h}_{2}^{\top} \boldsymbol{S}^{-1} \boldsymbol{h}_{2}\right\}\right)_{T \in \mathbb{R}_{+}}
$$

is a martingale, hence

$$
E_{2}=\mathbb{E}\left(\frac{\mathrm{d} \mathbb{P}_{\boldsymbol{\theta}+\boldsymbol{r} \boldsymbol{h}, 1}}{\mathrm{~d} \mathbb{P}_{\boldsymbol{\theta}, 1}}(\mathcal{Y}, \mathcal{X})\right)=\mathbb{E}\left(\frac{\mathrm{d} \mathbb{P}_{\boldsymbol{\theta}+\boldsymbol{r} \boldsymbol{h}, 0}}{\mathrm{~d} \mathbb{P}_{\boldsymbol{\theta}, 0}}(\mathcal{Y}, \mathcal{X})\right)=1
$$

and we conclude that the family $\left(\mathcal{E}_{T}\right)_{T \in \mathbb{R}++}$ of statistical experiments is LAQ at $\boldsymbol{\theta}$.

2.3.11 Remark. If $\boldsymbol{\theta}_{0}=\left(a_{0}, \alpha_{0}, b_{0}, \beta_{0}\right)$ with $a_{0} \in\left(\frac{\sigma_{1}^{2}}{2}, \infty\right), b_{0}=0$ and $\alpha_{0}, \beta_{0} \in \mathbb{R}$, then applying Theorem 2.2 .3 for the functions $\psi_{1}(a, \alpha, b, \beta):=a-a_{0}$ and $\psi_{2}(a, \alpha, b, \beta):=\alpha-\alpha_{0}$, $(a, \alpha, b, \beta) \in \mathbb{R}_{++} \times \mathbb{R}^{3}$, one can obtain that the family of tests that reject for values

$$
\begin{aligned}
S_{\boldsymbol{\theta}_{0}, T}^{(1)} & :=\frac{\sqrt{2 a_{0}-\sigma_{1}^{2}}}{\sigma_{1} \sqrt{2 \log T}} \int_{0}^{T} \frac{\mathrm{d} Y_{s}-\left(a_{0}-b_{0} Y_{s}\right) \mathrm{d} s}{Y_{s}} \\
S_{\boldsymbol{\theta}_{0}, T}^{(2)} & :=\frac{\sqrt{2 a_{0}-\sigma_{1}^{2}}}{\sigma_{2} \sqrt{2 \log T}} \int_{0}^{T} \frac{\mathrm{d} X_{s}-\left(\alpha_{0}-\beta_{0} Y_{s}\right) \mathrm{d} s}{Y_{s}},
\end{aligned}
$$

exceeding $z_{\alpha}$, respectively, are asymptotically optimal for testing $H_{0}^{(1)}: a \leqslant a_{0}$ against $H_{1}^{(1)}: a>a_{0}$, and $H_{0}^{(2)}: \alpha \leqslant \alpha_{0}$ against $H_{1}^{(2)}: \alpha>\alpha_{0}$, respectively. Indeed, $\left(\boldsymbol{J}_{\boldsymbol{\theta}_{0}}^{(1)}\right)^{-1}=$ $\left(a_{0}-\frac{\sigma_{1}^{2}}{2}\right) \boldsymbol{S}$

$$
\begin{aligned}
\boldsymbol{\Delta}_{\boldsymbol{\theta}_{0}, T} & =\left(\left[\begin{array}{cc}
\frac{1}{\sqrt{\log T}} & 0 \\
0 & \frac{1}{T}
\end{array}\right] \otimes\left[\begin{array}{cc}
\sigma_{1} & \sigma_{2} \varrho \\
0 & \sigma_{2} \sqrt{1 . \varrho^{2}}
\end{array}\right]^{-1}\right) \int_{0}^{T}\left(\left[\begin{array}{c}
\frac{1}{\sqrt{Y_{s}}} \\
-\sqrt{Y_{s}}
\end{array}\right] \otimes\left[\begin{array}{l}
\mathrm{d} W_{s} \\
\mathrm{~d} B_{s}
\end{array}\right]\right) \\
& =\int_{0}^{T}\left(\left[\begin{array}{cc}
\frac{1}{\sqrt{\log T}} & 0 \\
0 & \frac{1}{T}
\end{array}\right]\left[\begin{array}{c}
\frac{1}{\sqrt{Y_{s}}} \\
-\sqrt{Y_{s}}
\end{array}\right]\right) \otimes\left(\left[\begin{array}{cc}
\sigma_{1} & \sigma_{2} \varrho \\
0 & \sigma_{2} \sqrt{1 . \varrho^{2}}
\end{array}\right]^{-1}\left[\begin{array}{l}
\mathrm{d} W_{s} \\
\mathrm{~d} B_{s}
\end{array}\right]\right) \\
& =\int_{0}^{T}\left[\begin{array}{c}
\frac{1}{\sqrt{Y_{s} \log T}}-\frac{\sqrt{Y_{s}}}{T}
\end{array}\right] \otimes\left(\boldsymbol{S}^{-1} \frac{1}{\sqrt{Y_{s}}}\left[\begin{array}{l}
\mathrm{d} Y_{s}-\left(a_{0}-b_{0} Y_{s}\right) \mathrm{d} s \\
\mathrm{~d} X_{s}-\left(\alpha_{0}-\beta_{0} Y_{s}\right) \mathrm{d} s
\end{array}\right]\right),
\end{aligned}
$$

where we used

$$
\left[\begin{array}{cc}
\sigma_{1} & \sigma_{2} \varrho \\
0 & \sigma_{2} \sqrt{1 . \varrho^{2}}
\end{array}\right]^{-1}\left[\begin{array}{l}
\mathrm{d} W_{s} \\
\mathrm{~d} B_{s}
\end{array}\right]=\boldsymbol{S}^{-1}\left[\begin{array}{cc}
\sigma_{1} & 0 \\
\sigma_{2} \varrho & \sigma_{2} \sqrt{1 . \varrho^{2}}
\end{array}\right]\left[\begin{array}{l}
\mathrm{d} W_{s} \\
\mathrm{~d} B_{s}
\end{array}\right]=\boldsymbol{S}^{-1} \frac{1}{\sqrt{Y_{s}}}\left[\begin{array}{c}
\mathrm{d} Y_{s}-\left(a_{0}-b_{0} Y_{s}\right) \mathrm{d} s \\
\mathrm{~d} X_{s}-\left(\alpha_{0}-\beta_{0} Y_{s}\right) \mathrm{d} s
\end{array}\right],
$$


following from $(2.3 .6)$, thus

$$
\boldsymbol{\Delta}_{\boldsymbol{\theta}_{0}, T}^{-1}=\frac{1}{Y_{s} \sqrt{\log T}} \boldsymbol{S}^{-1} \int_{0}^{T}\left[\begin{array}{c}
\mathrm{d} Y_{s}-\left(a_{0}-b_{0} Y_{s}\right) \mathrm{d} s \\
\mathrm{~d} X_{s}-\left(\alpha_{0}-\beta_{0} Y_{s}\right) \mathrm{d} s
\end{array}\right],
$$

hence

$$
\left(\boldsymbol{J}_{\boldsymbol{\theta}_{0}}^{(1)}\right)^{-1} \boldsymbol{\Delta}_{\boldsymbol{\theta}_{0}, T}^{-1}=\left(a_{0}-\frac{\sigma_{1}^{2}}{2}\right) \frac{1}{\sqrt{\log T}} \int_{0}^{T} \frac{1}{Y_{s}}\left[\begin{array}{c}
\mathrm{d} Y_{s}-\left(a_{0}-b_{0} Y_{s}\right) \mathrm{d} s \\
\mathrm{~d} X_{s}-\left(\alpha_{0}-\beta_{0} Y_{s}\right) \mathrm{d} s
\end{array}\right],
$$

and $\psi_{i}^{\prime}\left(a_{0}, \alpha_{0}, b_{0}, \beta_{0}\right)=\boldsymbol{e}_{i}, \quad i \in\{1,2\}$.

\subsubsection{Supercritical case}

2.3.12 Theorem. If $a \in\left[\frac{\sigma_{1}^{2}}{2}, \infty\right), b \in \mathbb{R}_{--}$, and $\alpha, \beta \in \mathbb{R}$, then the family $\left(\mathcal{E}_{T}\right)_{T \in \mathbb{R}_{++}}$of statistical experiments, given in (2.3.9), is not LAQ at $\boldsymbol{\theta}:=(a, \alpha, b, \beta)$ with scaling matrices

$$
\boldsymbol{r}_{\boldsymbol{\theta}, T}:=\left[\begin{array}{cc}
1 & 0 \\
0 & \mathrm{e}^{b T / 2}
\end{array}\right] \otimes \boldsymbol{I}_{2}, \quad T \in \mathbb{R}_{++},
$$

although

$$
\left(\boldsymbol{\Delta}_{\boldsymbol{\theta}, T}(Y, X), \boldsymbol{J}_{\boldsymbol{\theta}, T}(Y, X)\right) \stackrel{\mathcal{D}}{\longrightarrow}\left(\boldsymbol{\Delta}_{\boldsymbol{\theta}}, \boldsymbol{J}_{\boldsymbol{\theta}}\right) \quad \text { as } T \rightarrow \infty
$$

with

$$
\boldsymbol{\Delta}_{\boldsymbol{\theta}}:=\left(\boldsymbol{I}_{2} \otimes\left[\begin{array}{cc}
\sigma_{1} & \sigma_{2} \varrho \\
0 & \sigma_{2} \sqrt{1-\varrho^{2}}
\end{array}\right]^{-1}\right)\left[\begin{array}{c}
\sigma_{1}^{-1} \widetilde{\mathcal{V}} \\
Z_{1} \\
\left(-\frac{\tilde{\mathcal{Y}}_{-1 / b}}{b}\right)^{1 / 2} \boldsymbol{Z}_{2}
\end{array}\right], \quad \boldsymbol{J}_{\boldsymbol{\theta}}:=\left[\begin{array}{cc}
\int_{0}^{-1 / b} \tilde{\mathcal{Y}}_{u} \mathrm{~d} u & 0 \\
0 & -\frac{\tilde{\mathcal{Y}}_{-1 / b}}{b}
\end{array}\right] \otimes \boldsymbol{S}^{-1}
$$

where $\left(\widetilde{\mathcal{Y}}_{t}\right)_{t \in \mathbb{R}_{+}}$is a CIR process given by the $S D E$

$$
\mathrm{d} \widetilde{\mathcal{Y}}_{t}=a \mathrm{~d} t+\sigma_{1} \sqrt{\widetilde{\mathcal{Y}}_{t}} \mathrm{~d} \mathcal{W}_{t}, \quad t \in \mathbb{R}_{+}
$$

with initial value $\widetilde{\mathcal{Y}}_{0}=y_{0}$, where $\left(\mathcal{W}_{t}\right)_{t \in \mathbb{R}_{+}}$is a standard Wiener process,

$$
\widetilde{\mathcal{V}}:=\log \widetilde{\mathcal{Y}}_{-1 / b}-\log y_{0}-\left(a-\frac{\sigma_{1}^{2}}{2}\right) \int_{0}^{-1 / b} \widetilde{\mathcal{Y}}_{u} \mathrm{~d} u
$$

$Z_{1}$ is a 1-dimensional standard normally distributed random variable, $Z_{2}$ is a 2-dimensional standard normally distributed random vector such that $\left(\widetilde{\mathcal{Y}}_{-1 / b}, \int_{0}^{-1 / b} \widetilde{\mathcal{Y}}_{u} \mathrm{~d} u\right), Z_{1}$ and $Z_{2}$ are independent, and $\boldsymbol{S}$ is defined in (2.3.7). Moreover, (2.1.3) also holds, but (2.1.4) is not valid.

If $a \in\left(\frac{\sigma_{1}^{2}}{2}, \infty\right)$ and $\alpha \in \mathbb{R}$ are fixed, then the subfamily

$$
\left(C\left(\mathbb{R}_{+}, \mathbb{R}^{2}\right), \mathcal{B}\left(C\left(\mathbb{R}_{+}, \mathbb{R}^{2}\right)\right),\left\{\mathbb{P}_{\boldsymbol{\theta}, T}: b \in \mathbb{R}_{--}, \beta \in \mathbb{R}\right\}\right)_{T \in \mathbb{R}_{++}}
$$


of statistical experiments is $L A M N$ at $(b, \beta)$ with scaling matrices $\boldsymbol{r}_{\boldsymbol{\theta}, T}^{(2)}:=\mathrm{e}^{b T / 2} \boldsymbol{I}_{2}, \quad T \in \mathbb{R}_{++}$, and with

$$
\boldsymbol{\Delta}_{\boldsymbol{\theta}}^{(2)}:=\left(-\frac{\widetilde{\mathcal{Y}}_{-1 / b}}{b}\right)^{1 / 2}\left[\begin{array}{cc}
\sigma_{1} & \sigma_{2} \varrho \\
0 & \sigma_{2} \sqrt{1-\varrho^{2}}
\end{array}\right]^{-1} \boldsymbol{Z}_{2}, \quad \boldsymbol{J}_{\boldsymbol{\theta}}^{(2)}:=\left(-\frac{\widetilde{\mathcal{Y}}_{-1 / b}}{b}\right) \boldsymbol{S}^{-1}
$$

Consequently, the family $\left(C\left(\mathbb{R}_{+}, \mathbb{R}^{2}\right), \mathcal{B}\left(C\left(\mathbb{R}_{+}, \mathbb{R}^{2}\right)\right),\left\{\mathbb{P}_{\boldsymbol{\theta}+\mathrm{e}^{b T / 2} \boldsymbol{h}, T}: \boldsymbol{h}_{2} \in \mathbb{R}^{2}\right\}\right)_{T \in \mathbb{R}_{++}}$of statistical experiments converges to the statistical experiment $\left(\mathbb{R}^{2} \times \mathbb{R}^{2 \times 2}, \mathcal{B}\left(\mathbb{R}^{2} \times \mathbb{R}^{2 \times 2}\right),\left\{\mathcal{L}\left(\left(\Delta_{\boldsymbol{\theta}}^{(2)}+\right.\right.\right.\right.$ $\left.\left.\left.\left.\boldsymbol{J}_{\boldsymbol{\theta}}^{(2)} \boldsymbol{h}_{2}, \boldsymbol{J}_{\boldsymbol{\theta}}^{(2)}\right) \mid \mathbb{P}\right): \boldsymbol{h}_{2} \in \mathbb{R}^{2}\right\}\right)$ as $T \rightarrow \infty$, where $\boldsymbol{h}:=\left(0, \boldsymbol{h}_{2}\right)^{\top} \in \mathbb{R}^{4}$.

Proof. We have

$$
\begin{gathered}
\boldsymbol{\Delta}_{\boldsymbol{\theta}, T}(Y, X)=\left(\left[\begin{array}{cc}
1 & 0 \\
0 & \mathrm{e}^{b T / 2}
\end{array}\right] \otimes \boldsymbol{I}_{2}\right)\left(\boldsymbol{I}_{2} \otimes\left[\begin{array}{cc}
\sigma_{1} & \sigma_{2} \varrho \\
0 & \sigma_{2} \sqrt{1-\varrho^{2}}
\end{array}\right]^{-1}\right)\left[\begin{array}{c}
\int_{0}^{T} \frac{\mathrm{d} W_{s}}{\sqrt{Y_{s}}} \\
\int_{0}^{T} \frac{\mathrm{d} B_{s}}{\sqrt{Y_{s}}} \\
-\int_{0}^{T} \sqrt{Y_{s}} \mathrm{~d} W_{s} \\
-\int_{0}^{T} \sqrt{Y_{s}} \mathrm{~d} B_{s}
\end{array}\right] \\
=\left(\left[\begin{array}{ll}
\left(\int_{0}^{T} \frac{\mathrm{d} s}{Y_{s}}\right)^{1 / 2} \\
0
\end{array}\right] \otimes\left[\begin{array}{cc}
\sigma_{1} & \sigma_{2} \varrho \\
0 & \sigma_{2} \sqrt{1-\varrho^{2}}
\end{array}\right]^{-1} \begin{array}{c}
\int_{0}^{T} \frac{\mathrm{d} W_{s}}{\sqrt{Y_{s}}} \\
\left(\int_{0}^{T} \frac{\mathrm{d} s}{Y_{s}}\right)^{1 / 2} \\
\frac{\int_{0}^{T} \frac{\mathrm{d} B_{s}}{\sqrt{Y_{s}}}}{\left(\int_{0}^{T} \frac{\mathrm{d} s}{Y_{s}}\right)^{1 / 2}} \\
-\frac{\int_{0}^{T} \sqrt{Y_{s}} \mathrm{~d} W_{s}}{\left(\int_{0}^{T} Y_{s} \mathrm{~d} s\right)^{1 / 2}} \\
-\frac{\int_{0}^{T} \sqrt{Y_{s}} \mathrm{~d} B_{s}}{\left(\int_{0}^{T} Y_{s} \mathrm{~d} s\right)^{1 / 2}}
\end{array}\right]
\end{gathered}
$$

and

$$
\begin{aligned}
\boldsymbol{J}_{\boldsymbol{\theta}, T}(Y, X) & =\left(\left[\begin{array}{cc}
1 & 0 \\
0 & \mathrm{e}^{b T / 2}
\end{array}\right] \otimes \boldsymbol{I}_{2}\right)\left(\left[\begin{array}{cc}
\int_{0}^{T} \frac{\mathrm{d} s}{Y_{s}} & -T \\
-T & \int_{0}^{T} Y_{s} \mathrm{~d} s
\end{array}\right] \otimes \boldsymbol{S}^{-1}\right)\left(\left[\begin{array}{cc}
1 & 0 \\
0 & \mathrm{e}^{b T / 2}
\end{array}\right] \otimes \boldsymbol{I}_{2}\right) \\
& =\left[\begin{array}{cc}
\int_{0}^{T} \frac{\mathrm{d} s}{Y_{s}} & -T \mathrm{e}^{b T / 2} \\
-T \mathrm{e}^{b T / 2} & \mathrm{e}^{b T} \int_{0}^{T} Y_{s} \mathrm{~d} s
\end{array}\right] \otimes \boldsymbol{S}^{-1} .
\end{aligned}
$$

We have

$$
\sigma_{1} \int_{0}^{T} \frac{\mathrm{d} W_{s}}{\sqrt{Y_{s}}}=\log Y_{T}-\log y_{0}+\left(\frac{\sigma_{1}^{2}}{2}-a\right) \int_{0}^{T} \frac{\mathrm{d} s}{Y_{s}}+b T, \quad T \in \mathbb{R}_{++} .
$$

see Barczy and Pap [7, Formula (4.10)]. Moreover,

$$
\mathrm{e}^{b T} Y_{T} \stackrel{\text { a.s. }}{\longrightarrow} V, \quad \mathrm{e}^{b T} \int_{0}^{T} Y_{s} \mathrm{~d} s \stackrel{\text { a.s. }}{\longrightarrow}-\frac{V}{b}, \quad \int_{0}^{T} \frac{\mathrm{d} s}{Y_{s}} \stackrel{\text { a.s. }}{\longrightarrow} \int_{0}^{\infty} \frac{\mathrm{d} s}{Y_{s}}, \quad \text { as } T \rightarrow \infty,
$$


see Barczy and Pap [7, Formulae (4.7) and (4.9)]. Thus,

$$
\frac{\sigma_{1} \int_{0}^{T} \frac{\mathrm{d} W_{s}}{\sqrt{Y_{s}}}}{\int_{0}^{T} \frac{\mathrm{d} s}{Y_{s}}}=\frac{\log \left(\mathrm{e}^{b T} Y_{T}\right)-\log y_{0}}{\int_{0}^{T} \frac{\mathrm{d} s}{Y_{s}}}+\frac{\sigma_{1}^{2}}{2}-a \stackrel{\text { a.s. }}{\longrightarrow} \frac{\log V-\log y_{0}}{\int_{0}^{\infty} \frac{\mathrm{d} s}{Y_{s}}}+\frac{\sigma_{1}^{2}}{2}-a
$$

as $T \rightarrow \infty$. By Theorem 4 in Ben Alaya and Kebaier [9],

$$
\left(V, \int_{0}^{\infty} \frac{\mathrm{d} s}{Y_{s}}\right) \stackrel{\mathcal{D}}{=}\left(\widetilde{\mathcal{Y}}_{-1 / b}, \int_{0}^{-1 / b} \widetilde{\mathcal{Y}}_{u} \mathrm{~d} u\right)
$$

hence

$$
\frac{\log V-\log y_{0}}{\int_{0}^{\infty} \frac{\mathrm{d} s}{Y_{s}}}+\frac{\sigma_{1}^{2}}{2}-a \stackrel{\mathcal{D}}{=} \frac{\log \widetilde{\mathcal{Y}}_{-1 / b}-\log y_{0}}{\int_{0}^{-1 / b} \widetilde{\mathcal{Y}}_{u} \mathrm{~d} u}+\frac{\sigma_{1}^{2}}{2}-a=\widetilde{\mathcal{V}}
$$

Further,

$$
\left(\frac{\int_{0}^{T} \frac{\mathrm{d} B_{s}}{\sqrt{Y_{s}}}}{\left(\int_{0}^{T} \frac{\mathrm{d} s}{Y_{s}}\right)^{1 / 2}}, \frac{\int_{0}^{T} \sqrt{Y_{s}} \mathrm{~d} W_{s}}{\left(\int_{0}^{T} Y_{s} \mathrm{~d} s\right)^{1 / 2}}, \frac{\int_{0}^{T} \sqrt{Y_{s}} \mathrm{~d} B_{s}}{\left(\int_{0}^{T} Y_{s} \mathrm{~d} s\right)^{1 / 2}}\right) \stackrel{\mathcal{D}}{\longrightarrow}\left(Z_{1},-Z_{2}\right) \quad \text { as } T \rightarrow \infty
$$

see Barczy and Pap [7, Formula (7.6)]. Hence we obtain (2.3.20).

It is known that under the condition $a \in\left[\frac{\sigma_{1}^{2}}{2}, \infty\right)$, we have $\mathbb{P}\left(\widetilde{\mathcal{Y}}_{-1 / b} \in \mathbb{R}_{++}\right)=1$ (see, e.g., page 442 in Revuz and Yor [42]) and $\mathbb{P}\left(\int_{0}^{-1 / b} \widetilde{\mathcal{Y}}_{s} \mathrm{~d} s \in \mathbb{R}_{++}\right)=1$ (which has been shown in the proof of Theorem 3.1 in Barczy et al. [5]), hence (2.1.3) holds. from

If $a \in\left(\frac{\sigma_{1}^{2}}{2}, \infty\right)$ and $\alpha \in \mathbb{R}$ are fixed, then LAMN property of the subfamily will follow

$$
\mathbb{E}\left(\exp \left\{\boldsymbol{h}_{2}^{\top} \boldsymbol{\Delta}_{\boldsymbol{\theta}}^{(2)}-\frac{1}{2} \boldsymbol{h}_{2}^{\top} \boldsymbol{J}_{\boldsymbol{\theta}}^{(2)} \boldsymbol{h}_{2}\right\}\right)=: E_{2}=1
$$

for all $\boldsymbol{h}_{2} \in \mathbb{R}^{2}$. We have

$$
\begin{gathered}
E_{2}=\mathbb{E}\left(\exp \left\{\left(-\frac{\widetilde{\mathcal{Y}}_{-1 / b}}{b}\right)^{-1 / 2} \boldsymbol{h}_{2}^{\top}\left[\begin{array}{cc}
\sigma_{1} & \sigma_{2} \varrho \\
0 & \sigma_{2} \sqrt{1-\varrho^{2}}
\end{array}\right]^{-1} \boldsymbol{Z}_{2}-\frac{1}{2}\left(-\frac{\widetilde{\mathcal{Y}}_{-1 / b}}{b}\right)^{-1} \boldsymbol{h}_{2}^{\top} \boldsymbol{S}^{-1} \boldsymbol{h}_{2}\right\}\right) \\
=\mathbb{E}\left(\mathbb { E } \left(\operatorname { e x p } \left\{\left(-\frac{\widetilde{\mathcal{Y}}_{-1 / b}}{b}\right)^{-1 / 2} \boldsymbol{h}_{2}^{\top}\left[\begin{array}{cc}
\sigma_{1} & \sigma_{2} \varrho \\
0 & \sigma_{2} \sqrt{1-\varrho^{2}}
\end{array}\right]^{-1} \boldsymbol{Z}_{2}\right.\right.\right. \\
\left.\left.\left.-\frac{1}{2}\left(-\frac{\widetilde{\mathcal{Y}}_{-1 / b}}{b}\right)^{-1} \boldsymbol{h}_{2}^{\top} \boldsymbol{S}^{-1} \boldsymbol{h}_{2}\right\} \mid \widetilde{\mathcal{Y}}_{-1 / b}\right)\right)=1
\end{gathered}
$$

by (2.3.19), thus we conclude (2.3.22).

Finally, we show that (2.1.4) is not valid for the whole family $\left(\mathcal{E}_{T}\right)_{T \in \mathbb{R}_{++}}$of statistical experiments, given in (2.3.9), i.e., there exists $\boldsymbol{h} \in \mathbb{R}^{4}$, such that

$$
\mathbb{E}\left(\exp \left\{\boldsymbol{h}^{\top} \boldsymbol{\Delta}_{\boldsymbol{\theta}}^{(2)}-\frac{1}{2} \boldsymbol{h}^{\top} \boldsymbol{J}_{\boldsymbol{\theta}}^{(2)} \boldsymbol{h}\right\}\right) \neq 1
$$


Indeed, using again (2.3.19), for $\boldsymbol{h}=(0,1,0)^{\top} \in \mathbb{R} \times \mathbb{R} \times \mathbb{R}^{2}$, we have

$$
\begin{aligned}
& \mathbb{E}\left(\exp \left\{\boldsymbol{h}^{\top} \boldsymbol{\Delta}_{\boldsymbol{\theta}}-\frac{1}{2} \boldsymbol{h}^{\top} \boldsymbol{J}_{\boldsymbol{\theta}} \boldsymbol{h}\right\}\right) \\
& =\mathbb{E}\left(\operatorname { e x p } \left\{\left[\begin{array}{l}
0 \\
1
\end{array}\right]^{\top}\left[\begin{array}{cc}
\sigma_{1} & \sigma_{2} \varrho \\
0 & \sigma_{2} \sqrt{1-\varrho^{2}}
\end{array}\right]^{-1}\left[\begin{array}{c}
\sigma_{1}^{-1} \widetilde{\mathcal{V}} \\
Z_{1}
\end{array}\right]\right.\right. \\
& \left.=\mathbb{E}\left(\exp \left\{\frac{1}{\sigma_{2} \sqrt{1-\varrho^{2}}} Z_{1}-\frac{1}{2 \sigma_{2}^{2}\left(1-\varrho^{2}\right)} \int_{0}^{-1 / b} \widetilde{\mathcal{Y}}_{u} \mathrm{~d} u\right)\left[\begin{array}{l}
0 \\
1
\end{array}\right]^{\top} \boldsymbol{S}^{-1}\left[\begin{array}{l}
0 \\
1
\end{array}\right]\right\}\right) \\
& =\mathbb{E}\left(\exp \left\{\frac{1}{\left.\left.\sigma_{2} \sqrt{1-\varrho_{u}^{2}} \mathrm{~d} u\right\}\right)} Z_{1}\right\}\right) \mathbb{E}\left(\exp \left\{-\frac{1}{2 \sigma_{2}^{2}\left(1-\varrho^{2}\right)} \int_{0}^{-1 / b} \widetilde{\mathcal{Y}}_{u} \mathrm{~d} u\right\}\right) \\
& =\exp \left\{\frac{1}{2 \sigma_{2}^{2}\left(1-\varrho^{2}\right)}\right\} \mathbb{E}\left(\exp \left\{-\frac{1}{2 \sigma_{2}^{2}\left(1-\varrho^{2}\right)} \int_{0}^{-1 / b} \widetilde{\mathcal{Y}}_{u} \mathrm{~d} u\right\}\right) \neq 1
\end{aligned}
$$

since, by Lemma 1 in Ben Alaya and Kebaier [8],

$$
\mathbb{E}\left(\exp \left\{-2 \mu^{2} \int_{0}^{t} \widetilde{\mathcal{Y}}_{u} \mathrm{~d} u\right\}\right)=\cosh \left(\sigma_{1} \mu t\right)^{-\frac{2 a}{\sigma_{1}^{2}}} \exp \left\{\frac{2 \mu y_{0}}{\sigma_{1}} \tanh \left(\sigma_{1} \mu t\right)\right\}
$$

for $\mu, t \in \mathbb{R}_{+}$. 



\section{Chapter 3}

\section{Statistical models for linear stochastic differential equation with time delay}

In this chapter the main results are presented. Assume that we have a stochastic process $(X(t))_{t \in[-r, T]}$, which satisfies the linear stochastic delay differential equation

$$
\mathrm{d} X(t)=\vartheta \int_{[-r, 0]} X(t+u) a(\mathrm{~d} u) \mathrm{d} t+\mathrm{d} W(t), \quad t \geqslant 0,
$$

where $a$ is a finite signed measure on $[-r, 0]$ and $W$ is a standard Wiener process. The real parameter $\vartheta$ is unknown, hence a good estimation from the continuous sample $(X(t))_{t \in[-r, T]}$ is needed. For this, the local asymptotic properties of the likelihood function are studied.

In Section 3.1 we state some useful results from the topic of delay differential equations. In Section 3.2 the main results for a special case are presented, where the delay is uniform on $[-1,0]$. Finally in Section 3.3 the general case is considered, where the delay measure $a$ is an arbitrary finite signed measure on $[-r, 0]$. The results for the uniform case have been published in Benke and Pap [10], and for the general case in Benke and Pap [11].

\subsection{Preliminaries for linear stochastic differential equa- tions with time delay}

Consider the linear stochastic delay differential equation (SDDE)

$$
\begin{cases}\mathrm{d} X(t)=\vartheta \int_{[-r, 0]} X(t+u) a(\mathrm{~d} u) \mathrm{d} t+\mathrm{d} W(t), & t \in \mathbb{R}_{+}, \\ X(t)=X_{0}(t), & t \in[-r, 0],\end{cases}
$$

where $r \in(0, \infty),(W(t))_{t \in \mathbb{R}_{+}}$is a standard Wiener process, $\vartheta \in \mathbb{R}$, and $a$ is a finite signed measure on $[-r, 0]$ with $a \neq 0$, and $\left(X_{0}(t)\right)_{t \in[-r, 0]}$ is a continuous process independent of $(W(t))_{t \in \mathbb{R}_{+}}$. We say that the SDDE (3.1.1) is a linear SDDE, because it can also be written 
in the form

$$
\begin{cases}\mathrm{d} X(t)=\vartheta F\left(X_{t}\right) \mathrm{d} t+\mathrm{d} W(t), & t \in \mathbb{R}_{+}, \\ X(t)=X_{0}(t), & t \in[-r, 0],\end{cases}
$$

where $F$ is a continuous and linear functional on $C([-r, 0], \mathbb{R})$ and $X_{t}$ is the segment of $X$ at time $t$, namely $X_{t}(u):=X(t+u)$, where $u \in[-r, 0]$. Indeed, by Riesz representation theorem, every continuous functional $F$ can be written in the form

$$
F(\psi)=\int_{[-r, 0]} \psi(u) a(\mathrm{~d} u)
$$

where $\psi \in C([-r, 0], \mathbb{R})$ cf., e.g., Appleby, Mao and Riedle [1].

The SDDE (3.1.1) can be written the integral form

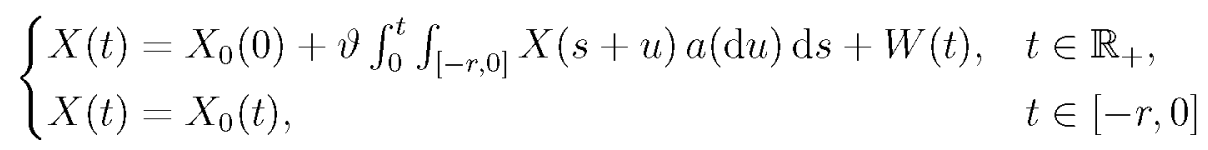

as well. Furthermore SDDE (3.1.1) is a special case of the affine stochastic delay differential equation

$$
\begin{cases}\mathrm{d} X(t)=\int_{-r}^{0} X(t+u) a_{\vartheta}(\mathrm{d} u) \mathrm{d} t+\mathrm{d} W(t), & t \in \mathbb{R}_{+}, \\ X(t)=X_{0}(t), & t \in[-r, 0]\end{cases}
$$

where $r>0$, and for each $\vartheta \in \Theta, a_{\vartheta}$ is a finite signed measure on $[-r, 0]$, see Gushchin and Küchler [19]. In that paper local asymptotic normality (LAN) has been proved for stationary solutions. In Gushchin and Küchler [16], the special case of (3.1.4) has been studied with $r=1$, $\Theta=\mathbb{R}^{2}$, and $a_{\vartheta}=\vartheta_{1} \delta_{0}+\vartheta_{2} \delta_{-1}$ for $\vartheta=\left(\vartheta_{1}, \vartheta_{2}\right)$, where $\delta_{x}$ denotes the Dirac measure concentrated at $x \in \mathbb{R}$, and they described the local properties of the likelihood function for the whole parameter space $\mathbb{R}^{2}$. In Section 3.2 a special case is studied, where $r=1$ and $a_{\vartheta}$ is the Lebesgue measure multiplied by $\vartheta \in \mathbb{R}$.

The solution $\left(X^{(\vartheta)}(t)\right)_{t \in \mathbb{R}_{+}}$of (3.1.1) exists (cf., e.g., Mao [35]), and obeys a variation of constants formula (see, Reiß, Riedle and van Gaans [41] or Gushchin and Küchler [19]). This implies that the solution is pathwise uniquely determined and can be represented as

$$
\begin{aligned}
X^{(\vartheta)}(t)= & x_{0, \vartheta}(t) X_{0}(0)+\vartheta \int_{[-r, 0]} \int_{u}^{0} x_{0, \vartheta}(t+u-s) X_{0}(s) \mathrm{d} s a(\mathrm{~d} u) \\
& +\int_{[0, t]} W(t-s) \mathrm{d} x_{0, \vartheta}(s), \quad t \in \mathbb{R}_{+},
\end{aligned}
$$

where $\left(x_{0, \vartheta}(t)\right)_{t \in[-r, \infty)}$ denotes the so-called fundamental solution of the deterministic homogeneous delay differential equation

$$
\left\{\begin{array}{lll}
x(t)=x_{0}(0)+\vartheta \int_{0}^{t} \int_{[-r, 0]} x(s+u) a(\mathrm{~d} u) \mathrm{d} s, & & t \in \mathbb{R}_{+}, \\
x(t)=x_{0}(t), & & t \in[-r, 0] .
\end{array}\right.
$$


with initial function

$$
x_{0}(t):= \begin{cases}0, & t \in[-r, 0), \\ 1, & t=0\end{cases}
$$

which means that $x_{0, \vartheta}$ is absolutely continuous on $\mathbb{R}_{+}, x_{0, \vartheta}(t)=0$ for $t \in[-r, 0)$, $x_{0, \vartheta}(0)=1$, and $\dot{x}_{0, \vartheta}(t)=\vartheta \int_{[-r, 0]} x_{0, \vartheta}(t+u) a(\mathrm{~d} u)$ for Lebesgue-almost all $t \in \mathbb{R}_{+}$. The domain of integration in the last integral in (3.1.5) includes zero, i.e.,

$$
\int_{[0, t]} W(t-s) \mathrm{d} x_{0, \vartheta}(s)=W(t)+\int_{(0, t]} W(t-s) \mathrm{d} x_{0, \vartheta}(s)=\int_{0}^{t} x_{0, \vartheta}(t-s) \mathrm{d} W(s), \quad t \in \mathbb{R}_{+} .
$$

Due to the variation of constants formula (3.1.5) to analyze the asymptotic behaviour of the solution, we have to know the asymptotic behaviour of the corresponding deterministic equation, thus the behaviour of the fundamental solution $x_{0}$. The following results can be found e.g., in Diekmann et al. [14] and in Hale [21] or see Gushchin and Küchler [19]. In the trivial case of $\vartheta=0$, we have $x_{0,0}(t)=1$ for all $t \in \mathbb{R}_{+}$, and $X^{(0)}(t)=X_{0}(0)+W(t)$ for all $t \in \mathbb{R}_{+}$. The asymptotic behaviour of $x_{0, \vartheta}(t)$ as $t \rightarrow \infty$ is connected with the so-called characteristic function $h_{\vartheta}: \mathbb{C} \rightarrow \mathbb{C}$, given by

$$
h_{\vartheta}(\lambda):=\lambda-\vartheta \int_{[-r, 0]} \mathrm{e}^{\lambda u} a(\mathrm{~d} u), \quad \lambda \in \mathbb{C},
$$

and the set $\Lambda_{\vartheta}$ of the (complex) solutions of the so-called characteristic equation for (3.1.6),

$$
\lambda-\vartheta \int_{[-r, 0]} \mathrm{e}^{\lambda u} a(\mathrm{~d} u)=0 .
$$

Note that a complex number $\lambda$ solves (3.1.8) if and only if $\left(\mathrm{e}^{\lambda t}\right)_{t \in[-r, \infty)}$ solves (3.1.6) with initial function $x_{0}(t)=\mathrm{e}^{\lambda t}, t \in[-r, 0]$. We have $\Lambda_{\vartheta} \neq \emptyset, \overline{\Lambda_{\vartheta}}=\Lambda_{\vartheta}$, and $\Lambda_{\vartheta}$ consists of isolated points. Moreover, $\Lambda_{\vartheta}$ is countably infinite except the case where $a$ is concentrated at 0 , or $\vartheta=0$. Further, for each $c \in \mathbb{R}$, the set $\left\{\lambda \in \Lambda_{\vartheta}: \operatorname{Re}(\lambda) \geqslant c\right\}$ is finite. In particular,

$$
v_{0}(\vartheta):=\sup \left\{\operatorname{Re}(\lambda): \lambda \in \Lambda_{\vartheta}\right\}<\infty
$$

For $\lambda \in \Lambda_{\vartheta}$, denote by $m_{\vartheta}(\lambda)$ the multiplicity of $\lambda$ as a solution of (3.1.8).

The Laplace transform of $\left(x_{0, \vartheta}(t)\right)_{t \in \mathbb{R}_{+}}$is given by

$$
\int_{0}^{\infty} \mathrm{e}^{-\lambda t} x_{0, \vartheta}(t) \mathrm{d} t=\frac{1}{h_{\vartheta}(\lambda)}, \quad \lambda \in \mathbb{C}, \quad \operatorname{Re}(\lambda)>v_{0}(\vartheta) .
$$

Based on the inverse Laplace transform and Cauchy's residue theorem, the following crucial lemma can be shown (see, e.g., Diekmann et al. [14, Lemma 5.1 and Theorem 5.4] or Gushchin and Küchler [17, Lemma 2.1]).

3.1.1 Lemma. For each $\vartheta \in \mathbb{R}$ and each $c \in \mathbb{R}$, the fundamental solution $\left(x_{0, \vartheta}(t)\right)_{t \in[-r, \infty)}$ of (3.1.6) can be represented in the form

$$
x_{0, \vartheta}(t)=\sum_{\substack{\lambda \in \Lambda_{\vartheta} \\ \operatorname{Re}(\lambda) \geqslant c}} \operatorname{Res}_{z=\lambda}\left(\frac{\mathrm{e}^{z t}}{h_{\vartheta}(z)}\right)+\psi_{\vartheta, c}(t)=\sum_{\substack{\lambda \in \Lambda_{\vartheta} \\ \operatorname{Re}(\lambda) \geqslant c}} p_{\vartheta, \lambda}(t) \mathrm{e}^{\lambda t}+\psi_{\vartheta, c}(t), \quad \text { as } t \rightarrow \infty,
$$


where $\psi_{\vartheta, c}: \mathbb{R}_{+} \rightarrow \mathbb{R}$ is a continuous function with $\psi_{\vartheta, c}(t)=\mathrm{o}\left(\mathrm{e}^{c t}\right)$ as $t \rightarrow \infty$, and for each $\vartheta \in \mathbb{R}$ and each $\lambda \in \Lambda_{\vartheta}, p_{\vartheta, \lambda}$ is a complex-valued polynomial of degree $m_{\vartheta}(\lambda)-1$ with $p_{\vartheta, \bar{\lambda}}=\overline{p_{\vartheta, \lambda}}$. More exactly,

$$
p_{\vartheta, \lambda}(t)=\sum_{\ell=0}^{m_{\vartheta}(\lambda)-1} \frac{A_{\vartheta,-1-\ell}(\lambda)}{\ell !} t^{\ell}
$$

where $A_{\vartheta, k}(\lambda), k \in\left\{-m_{\vartheta}(\lambda),-m_{\vartheta}(\lambda)+1, \ldots\right\}$ denotes the coefficients of the Laurent's series of $1 / h_{\vartheta}(z)$ at $z=\lambda$, i.e.,

$$
\frac{1}{h_{\vartheta}(z)}=\sum_{k=-m_{\vartheta}(\lambda)}^{\infty} A_{\vartheta, k}(\lambda)(z-\lambda)^{k}
$$

in a neighborhood of $\lambda$.

As a consequence, for any $c>v_{0}(\vartheta)$, we have $x_{0, \vartheta}(t)=\mathrm{o}\left(\mathrm{e}^{c t}\right)$ as $t \rightarrow \infty$. In particular, $\left(x_{0, \vartheta}(t)\right)_{t \in \mathbb{R}_{+}}$is square integrable if (and only if, see Gushchin and Küchler [17]) $v_{0}(\vartheta)<0$.

\subsection{The special case of uniformly distributed time delay}

In this section the main results in a special case are presented, when the delay is uniform. The results of this case can be derived from the general one, which are in Section 3.3. However the proofs are made in a same manner in the two cases, but the general one is more technical and complex. Hence this section can be useful to understand the proofs.

The other difference between the two cases, that in this uniformly delay case we have more information about the set $\Lambda_{\vartheta}$. This fact implies that we can determine the exact values of the parameter $\vartheta$, where the appropriate local asymptotic property is valid. In the general case we can add only a sufficient condition for this.

Assume $\left(X^{(\vartheta)}(t)\right)_{t \in \mathbb{R}_{+}}$is the solution of the SDDE

$$
\begin{cases}\mathrm{d} X(t)=\vartheta \int_{-1}^{0} X(t+u) \mathrm{d} u \mathrm{~d} t+\mathrm{d} W(t), & t \in \mathbb{R}_{+}, \\ X(t)=X_{0}(t), & t \in[-1,0] .\end{cases}
$$

Indeed, this equation is a special case of the $\operatorname{SDDE}(3.1 .1)$, where $r=1$, and $a$ is the Lebesgue measure on $[-1,0]$. This is, why we call this the uniformly distributed delay case. In the trivial case of $\vartheta=0$, we have $x_{0,0}(t)=1, t \in \mathbb{R}_{+}$, and $X^{(0)}(t)=X_{0}(0)+W(t)$, $t \in \mathbb{R}_{+}$. In case of $\vartheta \in \mathbb{R} \backslash\{0\}$, the characteristic function $h_{\vartheta}$, has the following form

$$
h_{\vartheta}(\lambda):=\lambda-\vartheta \int_{-1}^{0} \mathrm{e}^{\lambda u} \mathrm{~d} u, \quad \lambda \in \mathbb{C} .
$$

Applying usual methods (e.g., argument principle in complex analysis and the existence of local inverses of holomorphic functions), one can derive the following properties of the set $\Lambda_{\vartheta}$, see, 
e.g., Reiß [38]. Let $v_{0}(\vartheta)$ be as before,

$$
v_{0}(\vartheta):=\sup \left\{\operatorname{Re}(\lambda): \lambda \in \Lambda_{\vartheta}\right\}
$$

and put

$$
v_{1}(\vartheta):=\sup \left\{\operatorname{Re}(\lambda): \lambda \in \Lambda_{\vartheta}, \operatorname{Re}(\lambda)<v_{0}(\vartheta)\right\},
$$

where $\sup \emptyset:=-\infty$. We have the following cases:

(i) If $\vartheta \in\left(-\frac{\pi^{2}}{2}, 0\right)$ then $v_{0}(\vartheta)<0$;

(ii) If $\vartheta=-\frac{\pi^{2}}{2}$ then $v_{0}(\vartheta)=0$ and $v_{0}(\vartheta) \notin \Lambda_{\vartheta}$;

(iii) If $\vartheta \in\left(-\infty,-\frac{\pi^{2}}{2}\right)$ then $v_{0}(\vartheta)>0$ and $v_{0}(\vartheta) \notin \Lambda_{\vartheta}$;

(iv) If $\vartheta \in(0, \infty)$ then $v_{0}(\vartheta)>0, v_{0}(\vartheta) \in \Lambda_{\vartheta}, m\left(v_{0}(\vartheta)\right)=1$ and $v_{1}(\vartheta)<0$.

The Lemma 3.1.1 has now the following form.

3.2.1 Lemma. For each $\vartheta \in \mathbb{R} \backslash\{0\}$ and each $c \in\left(-\infty, v_{0}(\vartheta)\right)$, there exists $\gamma \in(-\infty, c)$ such that the fundamental solution $\left(x_{0, \vartheta}(t)\right)_{t \in[-1, \infty)}$ of $(3.1 .6)$ can be represented in the form

$$
x_{0, \vartheta}(t)=\xi_{0, \vartheta}(t) \mathrm{e}^{v_{0}(\vartheta) t}+\sum_{\substack{\lambda \in \Lambda_{\vartheta} \\ \operatorname{Re}(\lambda) \in\left[c, v_{0}(\vartheta)\right)}} c_{\vartheta}(\lambda) \mathrm{e}^{\lambda t}+\mathrm{o}\left(\mathrm{e}^{\gamma t}\right), \quad \text { as } t \rightarrow \infty,
$$

with some constants $c_{\vartheta}(\lambda), \lambda \in \Lambda_{\vartheta}$, and with

$$
\xi_{0, \vartheta}(t):= \begin{cases}\frac{v_{0}(\vartheta)}{v_{0}(\vartheta)^{2}+2 v_{0}(\vartheta)-\vartheta}, & \text { if } v_{0}(\vartheta) \in \Lambda_{\vartheta} \quad \text { and } m\left(v_{0}(\vartheta)\right)=1, \\ A_{0}(\vartheta) \cos \left(\kappa_{0}(\vartheta) t\right)+B_{0}(\vartheta) \sin \left(\kappa_{0}(\vartheta) t\right) & \text { if } v_{0}(\vartheta) \notin \Lambda_{\vartheta},\end{cases}
$$

with $\kappa_{0}(\vartheta):=\left|\operatorname{Im}\left(\lambda_{0}(\vartheta)\right)\right|$, where $\lambda_{0}(\vartheta) \in \Lambda_{\vartheta}$ is given by $\operatorname{Re}\left(\lambda_{0}(\vartheta)\right)=v_{0}(\vartheta)$, and

$$
\begin{aligned}
& A_{0}(\vartheta):=\frac{2\left[\left(v_{0}(\vartheta)^{2}-\kappa_{0}(\vartheta)^{2}\right)\left(v_{0}(\vartheta)-2\right)-\vartheta v_{0}(\vartheta)\right]}{\left(v_{0}(\vartheta)^{2}-\kappa_{0}(\vartheta)^{2}+2 v_{0}(\vartheta)-\vartheta\right)^{2}+4 \kappa_{0}(\vartheta)^{2}\left(v_{0}(\vartheta)+1\right)^{2}}, \\
& B_{0}(\vartheta):=\frac{2\left(v_{0}(\vartheta)^{2}+\kappa_{0}(\vartheta)^{2}+\vartheta\right) \kappa_{0}(\vartheta)}{\left(v_{0}(\vartheta)^{2}-\kappa_{0}(\vartheta)^{2}+2 v_{0}(\vartheta)-\vartheta\right)^{2}+4 \kappa_{0}(\vartheta)^{2}\left(v_{0}(\vartheta)+1\right)^{2}}
\end{aligned}
$$

\subsubsection{Radon-Nikodym derivatives}

From this section, we consider the SDDE (3.2.1) with fixed continuous initial process $\left(X_{0}(t)\right)_{t \in[-1,0]}$. Further, for all $T \in \mathbb{R}_{++}$, let $\mathbb{P}_{\vartheta, T}$ be the probability measure induced by $\left(X^{(\vartheta)}(t)\right)_{t \in[-1, T]}$ on $(C([-1, T], \mathbb{R}), \mathcal{B}(C([-1, T], \mathbb{R})))$. In order to calculate Radon-Nikodym

derivatives $\frac{d \mathbb{P}_{\theta, T}}{d \mathbb{P}_{\vartheta, T}}$ for certain $\theta, \vartheta \in \mathbb{R}$, we need the following statement, which can be derived from formula (7.139) in Section 7.6.4 of Liptser and Shiryaev [33]. 
3.2.2 Lemma. Let $\theta, \vartheta \in \mathbb{R}$. Then for all $T \in \mathbb{R}_{++}$, the measures $\mathbb{P}_{\theta, T}$ and $\mathbb{P}_{\vartheta, T}$ are absolutely continuous with respect to each other, and

$$
\begin{aligned}
& \log \frac{\mathrm{d} \mathbb{P}_{\theta, T}}{\mathrm{dP}_{\vartheta}(X}\left(\left.X^{(\vartheta)}\right|_{[-1, T]}\right) \\
& =(\theta-\vartheta) \int_{0}^{T} \int_{-1}^{0} X^{(\vartheta)}(t+u) \mathrm{d} u \mathrm{~d} X^{(\vartheta)}(t)-\frac{\theta^{2}-\vartheta^{2}}{2} \int_{0}^{T}\left(\int_{-1}^{0} X^{(\vartheta)}(t+u) \mathrm{d} u\right)^{2} \mathrm{~d} t \\
& =(\theta-\vartheta) \int_{0}^{T} \int_{-1}^{0} X^{(\vartheta)}(t+u) \mathrm{d} u \mathrm{~d} W(t)-\frac{(\theta-\vartheta)^{2}}{2} \int_{0}^{T}\left(\int_{-1}^{0} X^{(\vartheta)}(t+u) \mathrm{d} u\right)^{2} \mathrm{~d} t .
\end{aligned}
$$

In order to investigate convergence of the family

$$
\left(\mathcal{E}_{T}\right)_{T \in \mathbb{R}_{++}}:=\left(C\left(\mathbb{R}_{+}, \mathbb{R}\right), \mathcal{B}\left(C\left(\mathbb{R}_{+}, \mathbb{R}\right)\right),\left\{\mathbb{P}_{\vartheta, T}: \vartheta \in \mathbb{R}\right\}\right)_{T \in \mathbb{R}_{++}}
$$

of statistical experiments, we derive the following corollary.

3.2.3 Corollary. For each $\vartheta \in \mathbb{R}, T \in \mathbb{R}_{++}, r_{\vartheta, T} \in \mathbb{R}$ and $h_{T} \in \mathbb{R}$, we have

$$
\log \frac{\mathrm{d} \mathbb{P}_{\vartheta+r_{\vartheta, T} h_{T}, T}}{d \mathbb{P}_{\vartheta, T}}\left(\left.X^{(\vartheta)}\right|_{[-1, T]}\right)=h_{T} \Delta_{\vartheta, T}-\frac{1}{2} h_{T}^{2} J_{\vartheta, T},
$$

with

$$
\Delta_{\vartheta, T}:=r_{\vartheta, T} \int_{0}^{T} \int_{-1}^{0} X^{(\vartheta)}(t+u) \mathrm{d} u \mathrm{~d} W(t), \quad J_{\vartheta, T}:=r_{\vartheta, T}^{2} \int_{0}^{T}\left(\int_{-1}^{0} X^{(\vartheta)}(t+u) \mathrm{d} u\right)^{2} \mathrm{~d} t .
$$

Consequently, the quadratic approximation (2.1.1) in the definition of $L A Q$ is valid.

\subsubsection{Local asymptotics of the likelihood ratios}

3.2.4 Proposition. If $\vartheta \in\left(-\frac{\pi^{2}}{2}, 0\right)$ then the family $\left(\mathcal{E}_{T}\right)_{T \in \mathbb{R}_{++}}$of statistical experiments given in (3.2.3) is LAN at $\vartheta$ with scaling $r_{\vartheta, T}=\frac{1}{\sqrt{T}}, T \in \mathbb{R}_{++}$, and with

$$
J_{\vartheta}=\int_{0}^{\infty}\left(\int_{-1}^{0} x_{0, \vartheta}(t+u) \mathrm{d} u\right)^{2} \mathrm{~d} t
$$

3.2.5 Proposition. The family $\left(\mathcal{E}_{T}\right)_{T \in \mathbb{R}_{++}}$of statistical experiments given in (3.2.3) is $L A Q$ at 0 with scaling $r_{0, T}=\frac{1}{T}, T \in \mathbb{R}_{++}$, and with

$$
\Delta_{0}=\int_{0}^{1} \mathcal{W}(t) \mathrm{d} \mathcal{W}(t), \quad J_{0}=\int_{0}^{1} \mathcal{W}(t)^{2} \mathrm{~d} t
$$

where $(\mathcal{W}(t))_{t \in[0,1]}$ is a standard Wiener process. 
3.2.6 Proposition. The family $\left(\mathcal{E}_{T}\right)_{T \in \mathbb{R}_{++}}$of statistical experiments given in $(3.2 .3)$ is $L A Q$ at $-\frac{\pi^{2}}{2}$ with scaling $r_{-\frac{\pi^{2}}{2}, T}=\frac{1}{T}, T \in \mathbb{R}_{++}$, and with

$$
\begin{gathered}
\Delta_{-\frac{\pi^{2}}{2}}=\frac{16 \int_{0}^{1}\left(\mathcal{W}_{1}(s) \mathrm{d} \mathcal{W}_{2}(s)-\mathcal{W}_{2}(s) \mathrm{d} \mathcal{W}_{1}(s)\right)-4 \pi \int_{0}^{1}\left(\mathcal{W}_{1}(s) \mathrm{d} \mathcal{W}_{1}(s)+\mathcal{W}_{2}(s) \mathrm{d} \mathcal{W}_{2}(s)\right)}{\pi\left(\pi^{2}+16\right)}, \\
J_{-\frac{\pi^{2}}{2}}=\frac{16}{\pi^{2}\left(\pi^{2}+16\right)} \int_{0}^{1}\left(\mathcal{W}_{1}(t)^{2}+\mathcal{W}_{2}(t)^{2}\right) \mathrm{d} t
\end{gathered}
$$

where $\left(\mathcal{W}_{1}(t), \mathcal{W}_{2}(t)\right)_{t \in[0,1]}$ is a 2-dimensional standard Wiener process.

3.2.7 Proposition. If $\vartheta \in(0, \infty)$ then the family $\left(\mathcal{E}_{T}\right)_{T \in \mathbb{R}_{++}}$of statistical experiments given in (3.2.3) is LAMN at $\vartheta$ with scaling $r_{\vartheta, T}=\mathrm{e}^{-v_{0}(\vartheta) T}, T \in \mathbb{R}_{++}$, and with

$$
\Delta_{\vartheta}=Z \sqrt{J_{\vartheta}}, \quad J_{\vartheta}=\frac{\left(1-\mathrm{e}^{-v_{0}(\vartheta)}\right)^{2}}{2 v_{0}(\vartheta)\left(v_{0}(\vartheta)^{2}+2 v_{0}(\vartheta)-\vartheta\right)^{2}}\left(U^{(\vartheta)}\right)^{2},
$$

with

$$
U^{(\vartheta)}=X_{0}(0)+\vartheta \int_{-1}^{0} \int_{u}^{0} \mathrm{e}^{-v_{0}(\vartheta)(s-u)} X_{0}(s) \mathrm{d} s \mathrm{~d} u+\int_{0}^{\infty} \mathrm{e}^{-v_{0}(\vartheta) s} \mathrm{~d} W(s),
$$

and $Z$ is a standard normally distributed random variable independent of $J_{\vartheta}$.

3.2.8 Proposition. If $\vartheta \in\left(-\infty,-\frac{\pi^{2}}{2}\right)$ then the family $\left(\mathcal{E}_{T}\right)_{T \in \mathbb{R}_{++}}$of statistical experiments given in (3.2.3) is PLAMN at $\vartheta$ with period $D=\frac{\pi}{\kappa_{0}(\vartheta)}$, with scaling $r_{\vartheta, T}=\mathrm{e}^{-v_{0}(\vartheta) T}$, $T \in \mathbb{R}_{++}$, and with

$$
\Delta_{\vartheta}(d)=Z \sqrt{J_{\vartheta}(d)}, \quad J_{\vartheta}(d)=\int_{0}^{\infty} \mathrm{e}^{-2 v_{0}(\vartheta) s}\left(V^{(\vartheta)}(d-s)\right)^{2} \mathrm{~d} s, \quad d \in\left[0, \frac{\pi}{\kappa_{0}(\vartheta)}\right)
$$

where

$$
\begin{aligned}
V^{(\vartheta)}(t):= & X_{0}(0) \varphi_{\vartheta}(t)+\vartheta \int_{-1}^{0} \int_{u}^{0} \varphi_{\vartheta}(t+u-s) \mathrm{e}^{-v_{0}(\vartheta)(s-u)} X_{0}(s) \mathrm{d} s \mathrm{~d} u \\
& +\int_{0}^{\infty} \varphi_{\vartheta}(t-s) \mathrm{e}^{-v_{0}(\vartheta) s} \mathrm{~d} W(s), \quad t \in \mathbb{R}_{+},
\end{aligned}
$$

with

$$
\varphi_{\vartheta}(t):=A_{0}(\vartheta) \cos \left(\kappa_{0}(\vartheta) t\right)+B_{0}(\vartheta) \sin \left(\kappa_{0}(\vartheta) t\right), \quad t \in \mathbb{R}
$$

and $Z$ is a standard normally distributed random variable independent of $J_{\vartheta}(d)$.

\subsubsection{Maximum likelihood estimates}

For fixed $T \in \mathbb{R}_{++}$, maximizing $\log \frac{\mathrm{d}_{\mathbb{P}_{\vartheta}, T}}{\mathrm{dP}_{0, T}}\left(\left.X^{(\vartheta)}\right|_{[-1, T]}\right)$ in $\vartheta \in \mathbb{R}$ gives the MLE of $\vartheta$ based on the observations $(X(t))_{t \in[-1, T]}$ having the form

$$
\widehat{\vartheta}_{T}=\frac{\int_{0}^{T} \int_{-1}^{0} X^{(\vartheta)}(t+u) \mathrm{d} u \mathrm{~d} X^{(\vartheta)}(t)}{\int_{0}^{T}\left(\int_{-1}^{0} X^{(\vartheta)}(t+u) \mathrm{d} u\right)^{2} \mathrm{~d} t}
$$


provided that $\int_{0}^{T}\left(\int_{-1}^{0} X^{(\vartheta)}(t+u) \mathrm{d} u\right)^{2} \mathrm{~d} t>0$. Using the SDDE (3.2.1), one can check that

$$
\widehat{\vartheta}_{T}-\vartheta=\frac{\int_{0}^{T} \int_{-1}^{0} X^{(\vartheta)}(t+u) \mathrm{d} u \mathrm{~d} W(t)}{\int_{0}^{T}\left(\int_{-1}^{0} X^{(\vartheta)}(t+u) \mathrm{d} u\right)^{2} \mathrm{~d} t}
$$

hence

$$
r_{\vartheta, T}^{-1}\left(\widehat{\vartheta}_{T}-\vartheta\right)=\frac{\Delta_{\vartheta, T}}{J_{\vartheta, T}}
$$

Using the results of Section 3.2.2 and the continuous mapping theorem, we obtain the following result.

3.2.9 Proposition. If $\vartheta \in\left(-\frac{\pi^{2}}{2}, 0\right)$ then

$$
\sqrt{T}\left(\widehat{\vartheta}_{T}-\vartheta\right) \stackrel{\mathcal{D}}{\longrightarrow} \mathcal{N}\left(0, J_{\vartheta}^{-1}\right) \quad \text { as } T \rightarrow \infty,
$$

where $J_{\vartheta}$ is given in Proposition 3.2.4.

If $\vartheta=0$ then

$$
T\left(\widehat{\vartheta}_{T}-\vartheta\right)=T \widehat{\vartheta}_{T} \stackrel{\mathcal{D}}{\longrightarrow} \frac{\int_{0}^{1} \mathcal{W}(t) \mathrm{d} \mathcal{W}(t)}{\int_{0}^{1} \mathcal{W}(t)^{2} \mathrm{~d} t} \quad \text { as } T \rightarrow \infty
$$

where $(\mathcal{W}(t))_{t \in[0,1]}$ is a standard Wiener process.

$$
\begin{aligned}
& \text { If } \vartheta=-\frac{\pi^{2}}{2} \text { then } \\
& T\left(\widehat{\vartheta}_{T}-\vartheta\right)=T\left(\widehat{\vartheta}_{T}+\frac{\pi^{2}}{2}\right) \\
& \stackrel{\mathcal{D}}{\longrightarrow} \frac{16 \pi \int_{0}^{1}\left(\mathcal{W}_{1}(t) \mathrm{d} \mathcal{W}_{2}(t)-\mathcal{W}_{2}(t) \mathrm{d} \mathcal{W}_{1}(t)\right)-4 \pi^{2} \int_{0}^{1}\left(\mathcal{W}_{1}(t) \mathrm{d} \mathcal{W}_{1}(t)+\mathcal{W}_{2}(t) \mathrm{d} \mathcal{W}_{2}(t)\right)}{16 \int_{0}^{1}\left(\mathcal{W}_{1}(t)^{2}+\mathcal{W}_{2}(t)^{2}\right) \mathrm{d} t}
\end{aligned}
$$

as $T \rightarrow \infty$, where $\left(\mathcal{W}_{1}(t), \mathcal{W}_{2}(t)\right)_{t \in[0,1]}$ is a 2-dimensional standard Wiener process.

If $\vartheta \in(0, \infty)$ then

$$
\mathrm{e}^{v_{0}(\vartheta) T}\left(\hat{\vartheta}_{T}-\vartheta\right) \stackrel{\mathcal{D}}{\longrightarrow} \frac{Z}{\sqrt{J_{\vartheta}}} \quad \text { as } T \rightarrow \infty
$$

where $J_{\vartheta}$ is given in Proposition 3.2.7, and $Z$ is a standard normally distributed random variable independent of $J_{\vartheta}$.

$$
\begin{aligned}
& \text { If } \vartheta \in\left(-\infty,-\frac{\pi^{2}}{2}\right) \text { then for each } d \in\left[0, \frac{\pi}{\kappa_{0}(\vartheta)}\right), \\
& \qquad \mathrm{e}^{v_{0}(\vartheta)\left(k \frac{\pi}{\kappa_{0}(\vartheta)}+d\right)}\left(\widehat{\vartheta}_{k \frac{\pi}{\kappa_{0}(\vartheta)}+d}-\vartheta\right) \stackrel{\mathcal{D}}{\longrightarrow} \frac{Z}{\sqrt{J_{\vartheta}(d)}} \quad \text { as } k \rightarrow \infty,
\end{aligned}
$$

where $J_{\vartheta}(d)$ is given in Proposition 3.2.8, and $Z$ is a standard normally distributed random variable independent of $J_{\vartheta}(d)$. 


\subsubsection{Proofs}

For each $\vartheta \in \mathbb{R}$ and each deterministic continuous function $(y(t))_{t \in \mathbb{R}_{+}}$, consider a continuous stochastic process $\left(Y^{(\vartheta)}(t)\right)_{t \in \mathbb{R}_{+}}$given by

$$
Y^{(\vartheta)}(t):=y(t) X_{0}(0)+\vartheta \int_{-1}^{0} \int_{u}^{0} y(t+u-s) X_{0}(s) \mathrm{d} s \mathrm{~d} u+\int_{0}^{t} y(t-s) \mathrm{d} W(s)
$$

for $t \in[1, \infty)$.

3.2.10 Lemma. Let $(y(t))_{t \in \mathbb{R}_{+}}$be a deterministic continuous function. Put

$$
Z(t):=\int_{-1}^{0} \int_{u}^{0} y(t+u-s) X_{0}(s) \mathrm{d} s \mathrm{~d} u, \quad t \in[1, \infty) .
$$

Then for each $T \in[1, \infty)$,

$$
\int_{1}^{T} Z(t)^{2} \mathrm{~d} t \leqslant \int_{-1}^{0} X_{0}(s)^{2} \mathrm{~d} s \int_{0}^{T} y(v)^{2} \mathrm{~d} v .
$$

Proof. For each $t \in[1, \infty)$, by Fubini's theorem,

$$
Z(t)=\int_{-1}^{0} X_{0}(s) \int_{-1}^{s} y(t+u-s) \mathrm{d} u \mathrm{~d} s=\int_{-1}^{0} X_{0}(s) \int_{t-s-1}^{t} y(v) \mathrm{d} v \mathrm{~d} s .
$$

By the Cauchy-Schwarz inequality,

$$
Z(t)^{2} \leqslant \int_{-1}^{0} X_{0}(s)^{2} \mathrm{~d} s \int_{-1}^{0}\left(\int_{t-s-1}^{t} y(v) \mathrm{d} v\right)^{2} \mathrm{~d} s .
$$

Consequently,

$$
\int_{1}^{T} Z(t)^{2} \mathrm{~d} t \leqslant \int_{-1}^{0} X_{0}(s)^{2} \mathrm{~d} s \int_{1}^{T} \int_{-1}^{0}\left(\int_{t-s-1}^{t} y(v) \mathrm{d} v\right)^{2} \mathrm{~d} s \mathrm{~d} t
$$

where

$$
\int_{1}^{T} \int_{-1}^{0}\left(\int_{t-s-1}^{t} y(v) \mathrm{d} v\right)^{2} \mathrm{~d} s \mathrm{~d} t=\int_{-1}^{0} \int_{1}^{T}\left(\int_{t-s-1}^{t} y(v) \mathrm{d} v\right)^{2} \mathrm{~d} t \mathrm{~d} s .
$$

Here

$$
\begin{aligned}
\int_{1}^{T}\left(\int_{t-s-1}^{t} y(v) \mathrm{d} v\right)^{2} \mathrm{~d} t & \leqslant \int_{1}^{T} \int_{t-s-1}^{t} y(v)^{2} \mathrm{~d} v \mathrm{~d} t=\int_{-s}^{T} y(v)^{2} \int_{v}^{v+s+1} \mathrm{~d} t \mathrm{~d} v \\
& \leqslant \int_{-s}^{T} y(v)^{2} \mathrm{~d} v \leqslant \int_{0}^{T} y(v)^{2} \mathrm{~d} v
\end{aligned}
$$

for all $s \in[-1,0]$, hence we obtain the statement. 
3.2.11 Lemma. Let $(y(t))_{t \in \mathbb{R}_{+}}$be a deterministic continuous function with $\int_{0}^{\infty} y(t)^{2} \mathrm{~d} t<\infty$. Then for each $\vartheta \in \mathbb{R}$,

$$
\begin{gathered}
\frac{1}{T} \int_{0}^{T} Y^{(\vartheta)}(t) \mathrm{d} t \stackrel{\mathbb{P}}{\longrightarrow} 0 \quad \text { as } \quad T \rightarrow \infty, \\
\frac{1}{T} \int_{0}^{T} Y^{(\vartheta)}(t)^{2} \mathrm{~d} t \stackrel{\mathbb{P}}{\longrightarrow} \int_{0}^{\infty} y(t)^{2} \mathrm{~d} t \quad \text { as } \quad T \rightarrow \infty .
\end{gathered}
$$

Proof. Applying Lemma 4.3 of Gushchin and Küchler [16] for the special case $X_{0}(s)=0$, $s \in[-1,0]$, one can obtain

$$
\begin{gathered}
\frac{1}{T} \int_{0}^{T} \int_{0}^{t} y(t-s) \mathrm{d} W(s) \mathrm{d} t \stackrel{\mathbb{P}}{\longrightarrow} 0 \quad \text { as } T \rightarrow \infty \\
\frac{1}{T} \int_{0}^{T}\left(\int_{0}^{t} y(t-s) \mathrm{d} W(s)\right)^{2} \mathrm{~d} t \stackrel{\mathbb{P}}{\longrightarrow} \int_{0}^{\infty} y(t)^{2} \mathrm{~d} t \quad \text { as } T \rightarrow \infty
\end{gathered}
$$

We have

$$
\frac{1}{T} \int_{0}^{T} Y^{(\vartheta)}(t) \mathrm{d} t=\frac{1}{T} \int_{0}^{1} Y^{(\vartheta)}(t) \mathrm{d} t+X_{0}(0) I_{1}(T)+\frac{\vartheta}{T} \int_{1}^{T} Z(t) \mathrm{d} t+\frac{1}{T} \int_{1}^{T} \int_{0}^{t} y(t-s) \mathrm{d} W(s) \mathrm{d} t
$$

for $T \in \mathbb{R}_{+}$, where $(Z(t))_{t \in \mathbb{R}_{+}}$is given in Lemma 3.2.10, and

$$
I_{1}(T):=\frac{1}{T} \int_{1}^{T} y(t) \mathrm{d} t, \quad T \in \mathbb{R}_{+}
$$

By Lemma 3.2.10,

$$
\begin{gathered}
\left|I_{1}(T)\right| \leqslant \sqrt{\int_{0}^{T} \frac{1}{T^{2}} \mathrm{~d} t \int_{1}^{T} y(t)^{2} \mathrm{~d} t}=\sqrt{\frac{1}{T} \int_{0}^{\infty} y(t)^{2} \mathrm{~d} t} \rightarrow 0 \quad \text { as } T \rightarrow \infty \\
\left|\frac{1}{T} \int_{1}^{T} Z(t) \mathrm{d} t\right| \leqslant \sqrt{\frac{1}{T} \int_{1}^{T} Z(t)^{2} \mathrm{~d} t} \leqslant \sqrt{\frac{1}{T} \int_{-1}^{0} X_{0}(s)^{2} \mathrm{~d} s \int_{0}^{\infty} y(v)^{2} \mathrm{~d} v \stackrel{\text { a.s. }}{\rightarrow} 0 \quad \text { as } T \rightarrow \infty,}
\end{gathered}
$$

hence we obtain the first statement. Moreover,

$$
\frac{1}{T} \int_{0}^{T} Y^{(\vartheta)}(t)^{2} \mathrm{~d} t=\frac{1}{T} \int_{0}^{1} Y^{(\vartheta)}(t)^{2} \mathrm{~d} t+I_{2}(T)+2 I_{3}(T)+\frac{1}{T} \int_{1}^{T}\left(\int_{0}^{t} y(t-s) \mathrm{d} W(s)\right)^{2} \mathrm{~d} t
$$

for $T \in \mathbb{R}_{+}$, where

$$
\begin{gathered}
I_{2}(T):=\frac{1}{T} \int_{1}^{T}\left(y(t) X_{0}(0)+\vartheta Z(t)\right)^{2} \mathrm{~d} t, \quad T \in \mathbb{R}_{+}, \\
I_{3}(T):=\frac{1}{T} \int_{1}^{T}\left(y(t) X_{0}(0)+\vartheta Z(t)\right)\left(\int_{0}^{t} y(t-s) \mathrm{d} W(s)\right) \mathrm{d} t, \quad T \in \mathbb{R}_{+} .
\end{gathered}
$$


Again by Lemma 3.2.10,

$$
\begin{aligned}
0 \leqslant I_{2}(T) & \leqslant \frac{1}{T} \int_{1}^{T} 2\left(y(t)^{2} X_{0}(0)^{2}+\vartheta^{2} Z(t)^{2}\right) \mathrm{d} t \\
& \leqslant \frac{2 X_{0}(0)^{2}}{T} \int_{0}^{\infty} y(t)^{2} \mathrm{~d} t+\frac{2 \vartheta^{2}}{T} \int_{-1}^{0} X_{0}(s)^{2} \mathrm{~d} s \int_{0}^{\infty} y(v)^{2} \mathrm{~d} v \stackrel{\text { a.s. }}{\longrightarrow} 0 \quad \text { as } T \rightarrow \infty
\end{aligned}
$$

and

$$
\begin{aligned}
\left|I_{3}(T)\right| & \leqslant \frac{2}{T} \sqrt{\int_{1}^{T}\left(y(t)^{2} X_{0}(0)^{2}+\vartheta^{2} Z(t)^{2}\right)^{2} \mathrm{~d} t \int_{1}^{T}\left(\int_{0}^{t} y(t-s) \mathrm{d} W(s)\right)^{2} \mathrm{~d} t} \\
& =2 \sqrt{\frac{I_{2}(T)}{T} \int_{1}^{T}\left(\int_{0}^{t} y(t-s) \mathrm{d} W(s)\right)^{2} \mathrm{~d} t} \stackrel{\mathbb{P}}{\longrightarrow} 0 \quad \text { as } T \rightarrow \infty
\end{aligned}
$$

hence we obtain the second statement.

3.2.12 Lemma. Let $w \in \mathbb{R}_{++}$and $y(t):=\mathrm{e}^{w t}, t \in \mathbb{R}_{+}$. Then for each $\vartheta \in \mathbb{R}$,

$$
\begin{gathered}
\mathrm{e}^{-w t} Y^{(\vartheta)}(t) \stackrel{\text { a.s. }}{\longrightarrow} U_{w}^{(\vartheta)}, \quad \text { as } t \rightarrow \infty, \\
\mathrm{e}^{-2 w T} \int_{0}^{T}\left(Y^{(\vartheta)}(t)\right)^{2} \mathrm{~d} t \stackrel{\text { a.s. }}{\longrightarrow} \frac{1}{2 w}\left(U_{w}^{(\vartheta)}\right)^{2}, \quad \text { as } T \rightarrow \infty,
\end{gathered}
$$

with

$$
U_{w}^{(\vartheta)}:=X_{0}(0)+\vartheta \int_{-1}^{0} \int_{u}^{0} \mathrm{e}^{w(u-s)} X_{0}(s) \mathrm{d} s \mathrm{~d} u+\int_{0}^{\infty} \mathrm{e}^{-w s} \mathrm{~d} W(s)
$$

Proof. For each $t \in[1, \infty)$, we have

$$
\mathrm{e}^{-w t} Y^{(\vartheta)}(t)=X_{0}(0)+\vartheta \int_{-1}^{0} \int_{u}^{0} \mathrm{e}^{w(u-s)} X_{0}(s) \mathrm{d} s \mathrm{~d} u+\int_{0}^{t} \mathrm{e}^{-w s} \mathrm{~d} W(s)
$$

hence we obtain the first convergence. The second convergence follows by L'Hôspital's rule.

3.2.13 Lemma. Let $w \in \mathbb{R}_{++}, \quad \kappa \in \mathbb{R}$, and $y(t):=\varphi(t) \mathrm{e}^{w t}, \quad t \in \mathbb{R}_{+}$, with $\varphi(t)=\cos (\kappa t)$, $t \in \mathbb{R}_{+}$, or $\varphi(t)=\sin (\kappa t), t \in \mathbb{R}_{+}$. Then for each $\vartheta \in \mathbb{R}$,

$$
\begin{gathered}
\mathrm{e}^{-w t} Y^{(\vartheta)}(t)-V_{w}^{(\vartheta)}(t) \stackrel{\text { a.s. }}{\longrightarrow} 0, \quad \text { as } t \rightarrow \infty, \\
\mathrm{e}^{-2 w T} \int_{0}^{T}\left(Y^{(\vartheta)}(t)\right)^{2} \mathrm{~d} t-\int_{0}^{\infty} \mathrm{e}^{-2 w t}\left(V_{w}^{(\vartheta)}(T-t)\right)^{2} \mathrm{~d} t \stackrel{\mathbb{P}}{\longrightarrow} 0, \quad \text { as } T \rightarrow \infty,
\end{gathered}
$$

with

$$
V_{w}^{(\vartheta)}(t):=X_{0}(0) \varphi(t)+\vartheta \int_{-1}^{0} \int_{u}^{0} \varphi(t+u-s) \mathrm{e}^{w(u-s)} X_{0}(s) \mathrm{d} s \mathrm{~d} u+\int_{0}^{\infty} \varphi(t-s) \mathrm{e}^{-w s} \mathrm{~d} W(s)
$$

for $t \in \mathbb{R}$. 
Proof. Note that for each $t \in[1, \infty)$,

$$
\mathrm{e}^{-w t} Y^{(\vartheta)}(t)-V_{w}^{(\vartheta)}(t)=-\int_{t}^{\infty} \varphi(t-s) \mathrm{e}^{-w s} \mathrm{~d} W(s)
$$

which obviously tends almost surely to zero as $t \rightarrow \infty$, hence we obtain the first convergence.

In order to prove the second convergence, observe that for each $T \in \mathbb{R}_{+}$,

$$
\int_{0}^{\infty} \mathrm{e}^{-2 w t}\left(V_{w}^{(\vartheta)}(T-t)\right)^{2} \mathrm{~d} t=\int_{0}^{T} \mathrm{e}^{-2 w t}\left(V_{w}^{(\vartheta)}(T-t)\right)^{2} \mathrm{~d} t+\int_{T}^{\infty} \mathrm{e}^{-2 w t}\left(V_{w}^{(\vartheta)}(T-t)\right)^{2} \mathrm{~d} t
$$

where

$$
\int_{0}^{T} \mathrm{e}^{-2 w t}\left(V_{w}^{(\vartheta)}(T-t)\right)^{2} \mathrm{~d} t=\int_{0}^{T} \mathrm{e}^{-2 w(T-t)}\left(V_{w}^{(\vartheta)}(t)\right)^{2} \mathrm{~d} t=\mathrm{e}^{-2 w T} \int_{0}^{T}\left(\mathrm{e}^{w t} V_{w}^{(\vartheta)}(t)\right)^{2} \mathrm{~d} t
$$

hence

$$
\begin{aligned}
& \mathrm{e}^{-2 w T} \int_{0}^{T}\left(Y^{(\vartheta)}(t)\right)^{2} \mathrm{~d} t-\int_{0}^{\infty} \mathrm{e}^{-2 w t}\left(V_{w}^{(\vartheta)}(T-t)\right)^{2} \mathrm{~d} t \\
& =\mathrm{e}^{-2 w T} \int_{0}^{T}\left[\left(Y^{(\vartheta)}(t)\right)^{2}-\left(\mathrm{e}^{w t} V_{w}^{(\vartheta)}(t)\right)^{2}\right] \mathrm{d} t-\int_{T}^{\infty} \mathrm{e}^{-2 w t}\left(V_{w}^{(\vartheta)}(T-t)\right)^{2} \mathrm{~d} t \\
& =I_{0}(T)+I_{1}(T)+2 I_{2}(T)-I_{3}(T)
\end{aligned}
$$

with

$$
\begin{aligned}
& I_{0}(T):=\mathrm{e}^{-2 w T} \int_{0}^{1}\left(Y^{(\vartheta)}(t)-\mathrm{e}^{w t} V_{w}^{(\vartheta)}(t)\right)^{2} \mathrm{~d} t \\
& I_{1}(T):=\mathrm{e}^{-2 w T} \int_{1}^{T}\left(Y^{(\vartheta)}(t)-\mathrm{e}^{w t} V_{w}^{(\vartheta)}(t)\right)^{2} \mathrm{~d} t \\
& I_{2}(T):=\mathrm{e}^{-2 w T} \int_{0}^{T}\left(Y^{(\vartheta)}(t)-\mathrm{e}^{w t} V_{w}^{(\vartheta)}(t)\right) \mathrm{e}^{w t} V_{w}^{(\vartheta)}(t) \mathrm{d} t \\
& I_{3}(T):=\int_{T}^{\infty} \mathrm{e}^{-2 w t}\left(V_{w}^{(\vartheta)}(T-t)\right)^{2} \mathrm{~d} t .
\end{aligned}
$$

The processes $\left(Y^{(\vartheta)}(t)\right)_{t \in \mathbb{R}_{+}}$and $\left(V_{w}^{(\vartheta)}(t)\right)_{t \in \mathbb{R}_{+}}$are continuous, hence

$$
\mathbb{E}\left(\left|I_{0}(T)\right|\right)=\mathrm{e}^{-2 w T} \int_{0}^{1} \mathbb{E}\left[\left(Y^{(\vartheta)}(t)-\mathrm{e}^{w t} V_{w}^{(\vartheta)}(t)\right)^{2}\right] \mathrm{d} t \rightarrow 0 \quad \text { as } T \rightarrow \infty,
$$

implying $I_{0}(T) \stackrel{\mathbb{P}}{\longrightarrow} 0$ as $T \rightarrow \infty$. By $(3.2 .5)$,

$$
I_{1}(T)=\mathrm{e}^{-2 w T} \int_{1}^{T} \mathrm{e}^{2 w t}\left(\int_{t}^{\infty} \varphi(t-s) \mathrm{e}^{-w s} \mathrm{~d} W(s)\right)^{2} \mathrm{~d} t
$$


hence

$$
\begin{aligned}
\mathbb{E}\left(\left|I_{1}(T)\right|\right) & =\mathrm{e}^{-2 w T} \int_{1}^{T} \mathrm{e}^{2 w t} \int_{t}^{\infty} \varphi(t-s)^{2} \mathrm{e}^{-2 w s} \mathrm{~d} s \mathrm{~d} t \leqslant \mathrm{e}^{-2 w T} \int_{1}^{T} \mathrm{e}^{2 w t} \int_{t}^{\infty} \mathrm{e}^{-2 w s} \mathrm{~d} s \mathrm{~d} t \\
& =\frac{T}{2 w} \mathrm{e}^{-2 w T} \rightarrow 0 \quad \text { as } T \rightarrow \infty
\end{aligned}
$$

implying $I_{1}(T) \stackrel{\mathbb{P}}{\longrightarrow} 0$ as $T \rightarrow \infty$. Moreover, by the Cauchy-Schwarz inequality,

$$
\left|I_{2}(T)\right| \leqslant \sqrt{I_{1}(T) \mathrm{e}^{-2 w T} \int_{0}^{T}\left(\mathrm{e}^{w t} V_{w}^{(\vartheta)}(t)\right)^{2} \mathrm{~d} t}
$$

with

$$
\mathrm{e}^{-2 w T} \int_{0}^{T}\left(\mathrm{e}^{w t} V_{w}^{(\vartheta)}(t)\right)^{2} \mathrm{~d} t=\int_{0}^{T} \mathrm{e}^{-2 w t} V_{w}^{(\vartheta)}(T-t)^{2} \mathrm{~d} t \leqslant \frac{1}{2 w} \sup _{t \in \mathbb{R}}\left(V_{w}^{(\vartheta)}(t)\right)^{2},
$$

where $\sup _{t \in \mathbb{R}}\left(V_{w}^{(\vartheta)}(t)\right)^{2}<\infty$ almost surely, since $\left(V_{w}^{(\vartheta)}(t)\right)_{t \in \mathbb{R}}$ is a continuous and periodic process. Consequently, $I_{2}(T) \stackrel{\mathbb{P}}{\longrightarrow} 0$ as $T \rightarrow \infty$. Finally,

$$
\left|I_{3}(T)\right| \leqslant \frac{\mathrm{e}^{-2 w T}}{2 w} \sup _{t \in \mathbb{R}}\left(V_{w}^{(\vartheta)}(t)\right)^{2} \stackrel{\text { a.s. }}{\longrightarrow} 0 \quad \text { as } T \rightarrow \infty,
$$

hence we obtain the second convergence of the statement.

Proof of Proposition 3.2.4. For each $t \in[1, \infty)$, by $(3.1 .5)$, we have

$$
\begin{aligned}
\int_{-1}^{0} X^{(\vartheta)}(t+u) \mathrm{d} u= & X_{0}(0) \int_{-1}^{0} x_{0, \vartheta}(t+u) \mathrm{d} u+\vartheta \int_{-1}^{0} \int_{-1}^{0} \int_{v}^{0} x_{0, \vartheta}(t+u+v-s) X_{0}(s) \mathrm{d} s \mathrm{~d} v \mathrm{~d} u \\
& +\int_{-1}^{0} \int_{0}^{t+u} x_{0, \vartheta}(t+u-s) \mathrm{d} W(s) \mathrm{d} u
\end{aligned}
$$

Here we have

$$
\begin{aligned}
\int_{-1}^{0} \int_{-1}^{0} \int_{v}^{0} x_{0, \vartheta}(t+u+v-s) X_{0}(s) \mathrm{d} s \mathrm{~d} v \mathrm{~d} u & =\int_{-1}^{0} \int_{-1}^{0} \int_{v}^{0} x_{0, \vartheta}(t+u+v-s) X_{0}(s) \mathrm{d} s \mathrm{~d} u \mathrm{~d} v \\
& =\int_{-1}^{0} \int_{v}^{0} X_{0}(s) \int_{-1}^{0} x_{0, \vartheta}(t+u+v-s) \mathrm{d} u \mathrm{~d} s \mathrm{~d} v
\end{aligned}
$$

and

$$
\begin{aligned}
& \int_{-1}^{0} \int_{0}^{t+u} x_{0, \vartheta}(t+u-s) \mathrm{d} W(s) \mathrm{d} u \\
& =\int_{0}^{t-1} \int_{-1}^{0} x_{0, \vartheta}(t+u-s) \mathrm{d} u \mathrm{~d} W(s)+\int_{t-1}^{t} \int_{s-t}^{0} x_{0, \vartheta}(t+u-s) \mathrm{d} u \mathrm{~d} W(s) \\
& =\int_{0}^{t} \int_{-1}^{0} x_{0, \vartheta}(t+u-s) \mathrm{d} u \mathrm{~d} W(s),
\end{aligned}
$$


since $t \in[1, \infty), s \in[t-1, t]$ and $u \in[-1, s-t)$ imply $t+u-s \in[-1,0)$, and hence $x_{0, \vartheta}(t+u-s)=0$. Consequently, the process $\left(\int_{-1}^{0} X^{(\vartheta)}(t+u) \mathrm{d} u\right)_{t \in \mathbb{R}_{+}}$has a representation $(3.2 .4)$ with

$$
y(t)=\int_{-1}^{0} x_{0, \vartheta}(t+u) \mathrm{d} u, \quad t \in \mathbb{R}_{+} .
$$

Assumption $\vartheta \in\left(-\frac{\pi^{2}}{2}, 0\right)$ implies $v_{0}(\vartheta)<0$, and hence $\int_{0}^{\infty} x_{0, \vartheta}(t)^{2} \mathrm{~d} t<\infty$ holds. Thus

$$
\begin{aligned}
\int_{1}^{\infty} y(t)^{2} \mathrm{~d} t & =\int_{1}^{\infty}\left(\int_{-1}^{0} x_{0, \vartheta}(t+u) \mathrm{d} u\right)^{2} \mathrm{~d} t \\
& =\int_{-1}^{0} \int_{-1}^{0} \int_{1}^{\infty} x_{0, \vartheta}(t+u) x_{0, \vartheta}(t+v) \mathrm{d} t \mathrm{~d} u \mathrm{~d} v \leqslant \int_{0}^{\infty} x_{0, \vartheta}(t)^{2} \mathrm{~d} t
\end{aligned}
$$

since

$$
\begin{aligned}
\left|\int_{1}^{\infty} x_{0, \vartheta}(t+u) x_{0, a}(t+v) \mathrm{d} t\right| & \leqslant \sqrt{\int_{1}^{\infty} x_{0, \vartheta}(t+u)^{2} \mathrm{~d} t \int_{1}^{\infty} x_{0, \vartheta}(t+v)^{2}} \mathrm{~d} t \\
& =\sqrt{\int_{1+u}^{\infty} x_{0, \vartheta}(s+u)^{2} \mathrm{~d} s \int_{1+v}^{\infty} x_{0, \vartheta}(s+v)^{2} \mathrm{~d} s} \leqslant \int_{0}^{\infty} x_{0, \vartheta}(t)^{2} \mathrm{~d} t .
\end{aligned}
$$

Consequently, $\quad \int_{0}^{\infty} y(t)^{2} \mathrm{~d} t \leqslant \int_{0}^{1} y(t)^{2} \mathrm{~d} t+\int_{0}^{\infty} x_{0, \vartheta}(t)^{2} \mathrm{~d} t<\infty$, thus one can apply Lemma 3.2 .11 to obtain

$$
J_{\vartheta, T}=\frac{1}{T} \int_{0}^{T}\left(\int_{-1}^{0} X^{(\vartheta)}(t+u) \mathrm{d} u\right)^{2} \mathrm{~d} t \stackrel{\mathbb{P}}{\longrightarrow} \int_{0}^{\infty}\left(\int_{-1}^{0} x_{0, \vartheta}(t+u) \mathrm{d} u\right)^{2} \mathrm{~d} t=J_{\vartheta}
$$

as $T \rightarrow \infty$. Moreover, the process

$$
M^{(\vartheta)}(T):=\int_{0}^{T} \int_{-1}^{0} X^{(\vartheta)}(t+u) \mathrm{d} u \mathrm{~d} W(t), \quad T \in \mathbb{R}_{+},
$$

is a continuous martingale with $M^{(\vartheta)}(0)=0$ and with quadratic variation

$$
\left\langle M^{(\vartheta)}\right\rangle(T)=\int_{0}^{T}\left(\int_{-1}^{0} X^{(\vartheta)}(t+u) \mathrm{d} u\right)^{2} \mathrm{~d} t
$$

hence, Theorem VIII.5.42 of Jacod and Shiryaev [25] yields the statement.

Proof of Proposition 3.2.5. We have

$$
\Delta_{0, T}=\frac{1}{T} \int_{0}^{T} \int_{-1}^{0} X^{(0)}(t+u) \mathrm{d} u \mathrm{~d} W(t), \quad T \in \mathbb{R}_{++} .
$$

As in the proof of Proposition 3.2.4, for each $t \in[1, \infty)$, one can obtain

$$
\int_{-1}^{0} X^{(0)}(t+u) \mathrm{d} u=X_{0}(0) \int_{-1}^{0} x_{0,0}(t+u) \mathrm{d} u+\int_{0}^{t} \int_{-1}^{0} x_{0,0}(t+u-s) \mathrm{d} u \mathrm{~d} W(s) .
$$


Here we have

$$
\int_{-1}^{0} x_{0,0}(t+u) \mathrm{d} u=1, \quad \int_{-1}^{0} x_{0,0}(t+u-s) \mathrm{d} u= \begin{cases}1, & \text { for } s \in[0, t-1] \\ t-s, & \text { for } s \in[t-1, t]\end{cases}
$$

hence

$$
\begin{aligned}
\int_{-1}^{0} X^{(0)}(t+u) \mathrm{d} u & =X_{0}(0)+\int_{0}^{t-1} \mathrm{~d} W(s)+\int_{t-1}^{t}(t-s) \mathrm{d} W(s) \\
& =X_{0}(0)+W(t)+\int_{t-1}^{t}(t-s-1) \mathrm{d} W(s)=W(t)+\bar{X}(t)
\end{aligned}
$$

where $\mathbb{E}\left(T^{-2} \int_{0}^{T} \bar{X}(t)^{2} \mathrm{~d} t\right) \rightarrow 0$ as $T \rightarrow \infty$. For each $T \in \mathbb{R}_{++}$, consider the process

$$
W^{T}(s):=\frac{1}{\sqrt{T}} W(T s), \quad s \in[0,1]
$$

Then we have

$$
\begin{aligned}
\Delta_{0, T} & =\int_{0}^{1} W^{T}(t) \mathrm{d} W^{T}(t)+\frac{1}{T} \int_{0}^{T} \bar{X}(t) \mathrm{d} W(t) \\
J_{0, T} & =\int_{0}^{1} W^{T}(t)^{2} \mathrm{~d} t+\frac{2}{T^{2}} \int_{0}^{T} W(t) \bar{X}(t) \mathrm{d} t+\frac{1}{T^{2}} \int_{0}^{T} \bar{X}(t)^{2} \mathrm{~d} t .
\end{aligned}
$$

Here

$$
\frac{1}{T} \int_{0}^{T} \bar{X}(t) \mathrm{d} W(t) \stackrel{\mathbb{P}}{\longrightarrow} 0, \quad \frac{1}{T^{2}} \int_{0}^{T} \bar{X}(t)^{2} \mathrm{~d} t \stackrel{\mathbb{P}}{\longrightarrow} 0
$$

as $T \rightarrow \infty$, since

$$
\mathbb{E}\left[\left(\frac{1}{T} \int_{0}^{T} \bar{X}(t) \mathrm{d} W(t)\right)^{2}\right]=\frac{1}{T^{2}} \int_{0}^{T} \mathbb{E}\left(\bar{X}(t)^{2}\right) \mathrm{d} t \rightarrow 0
$$

By the functional central limit theorem,

$$
W^{T} \stackrel{\mathcal{D}}{\longrightarrow} \mathcal{W} \quad \text { as } T \rightarrow \infty
$$

hence

$$
\begin{aligned}
\left|\frac{1}{T^{2}} \int_{0}^{T} W(t) \bar{X}(t) \mathrm{d} t\right| & \leqslant \sqrt{\left(\frac{1}{T^{2}} \int_{0}^{T} W(t)^{2} \mathrm{~d} t\right)\left(\frac{1}{T^{2}} \int_{0}^{T} \bar{X}(t)^{2} \mathrm{~d} t\right)} \\
& =\sqrt{\left(\int_{0}^{1} W^{T}(t)^{2} \mathrm{~d} t\right)\left(\frac{1}{T^{2}} \int_{0}^{T} \bar{X}(t)^{2} \mathrm{~d} t\right)} \stackrel{\mathbb{P}}{\rightarrow} 0 \quad \text { as } T \rightarrow \infty,
\end{aligned}
$$

and the claim follows from Corollary 4.12 in Gushchin and Küchler [16]. 
Proof of Proposition 3.2.6. We have

$$
\begin{array}{ll}
\Delta_{-\frac{\pi^{2}}{2}, T}=\frac{1}{T} \int_{0}^{T} \int_{-1}^{0} X^{\left(-\pi^{2} / 2\right)}(t+u) \mathrm{d} u \mathrm{~d} W(t), & T \in \mathbb{R}_{++}, \\
J_{-\frac{\pi^{2}}{2}, T}=\frac{1}{T^{2}} \int_{0}^{T}\left(\int_{-1}^{0} X^{\left(-\pi^{2} / 2\right)}(t+u) \mathrm{d} u\right)^{2} \mathrm{~d} t, \quad T \in \mathbb{R}_{++} .
\end{array}
$$

As in the proof of Proposition 3.2.4, for each $t \in[1, \infty)$, we have

$$
\begin{aligned}
\int_{-1}^{0} X^{\left(-\pi^{2} / 2\right)}(t+u) \mathrm{d} u= & X_{0}(0) \int_{-1}^{0} x_{0,-\frac{\pi^{2}}{2}}(t+u) \mathrm{d} u+\int_{0}^{t} \int_{-1}^{0} x_{0,-\frac{\pi^{2}}{2}}(t+u-s) \mathrm{d} u \mathrm{~d} W(s) \\
& -\frac{\pi^{2}}{2} \int_{-1}^{0} \int_{v}^{0} X_{0}(s) \int_{-1}^{0} x_{0,-\frac{\pi^{2}}{2}}(t+u+v-s) \mathrm{d} u \mathrm{~d} s \mathrm{~d} v .
\end{aligned}
$$

We have $v_{0}\left(-\frac{\pi^{2}}{2}\right)=0$ and $\kappa_{0}\left(-\frac{\pi^{2}}{2}\right)=\pi$, hence $A_{0}\left(-\frac{\pi^{2}}{2}\right)=\frac{16}{\pi^{2}+16}$ and $B_{0}\left(-\frac{\pi^{2}}{2}\right)=\frac{4 \pi}{\pi^{2}+16}$. Consequently, by Lemma 3.2.1, there exists $\gamma \in(-\infty, 0)$ such that

$$
x_{0,-\frac{\pi^{2}}{2}}(t)=\frac{16 \cos (\pi t)+4 \pi \sin (\pi t)}{\pi^{2}+16}+\mathrm{o}\left(\mathrm{e}^{\gamma t}\right), \quad \text { as } t \rightarrow \infty,
$$

and hence

$$
\begin{aligned}
\int_{-1}^{0} X^{\left(-\pi^{2} / 2\right)}(t+u) \mathrm{d} u & =\int_{0}^{t} \int_{-1}^{0} \frac{16 \cos (\pi(t+u-s))+4 \pi \sin (\pi(t+u-s))}{\pi^{2}+16} \mathrm{~d} u \mathrm{~d} W(s)+\bar{X}(t) \\
& =\int_{0}^{t} \frac{32 \sin (\pi(t-s))-8 \pi \cos (\pi(t-s))}{\pi\left(\pi^{2}+16\right)} \mathrm{d} W(s)+\bar{X}(t),
\end{aligned}
$$

where $T^{-2} \int_{0}^{T} \bar{X}(t)^{2} \mathrm{~d} t \stackrel{\mathbb{P}}{\longrightarrow} 0$ as $T \rightarrow \infty$. Introducing

$$
X_{1}(t):=\int_{0}^{t} \cos (\pi s) \mathrm{d} W(s), \quad X_{2}(t):=\int_{0}^{t} \sin (\pi s) \mathrm{d} W(s), \quad t \in \mathbb{R}_{+},
$$

we obtain

$$
\begin{aligned}
& \int_{-1}^{0} X^{\left(-\pi^{2} / 2\right)}(t+u) \mathrm{d} u \\
& \quad=\frac{32 X_{1}(t) \sin (\pi t)-32 X_{2}(t) \cos (\pi t)-8 \pi X_{1}(t) \cos (\pi t)-8 \pi X_{2}(t) \sin (\pi t)}{\pi\left(\pi^{2}+16\right)}+\bar{X}(t) .
\end{aligned}
$$

For each $T \in \mathbb{R}_{++}$, consider the following processes on $[0,1]$ :

$$
\begin{gathered}
W^{T}(s):=\frac{1}{\sqrt{T}} W(T s), \\
X_{1}^{T}(s):=\frac{1}{\sqrt{T}} X_{1}(T s)=\int_{0}^{s} \cos (\pi T s) \mathrm{d} W^{T}(s), \\
X_{2}^{T}(s):=\frac{1}{\sqrt{T}} X_{2}(T s)=\int_{0}^{s} \sin (\pi T s) \mathrm{d} W^{T}(s), \\
X^{T}(s):=\frac{32 X_{1}^{T}(s) \sin (\pi T s)-32 X_{2}^{T}(s) \cos (\pi T s)-8 \pi X_{1}(s) \cos (\pi T s)-8 \pi X_{2}(s) \sin (\pi T s)}{\pi\left(\pi^{2}+16\right)} .
\end{gathered}
$$


Then, for each $T \in \mathbb{R}_{++}$, we have

$$
\int_{-1}^{0} X^{\left(-\pi^{2} / 2\right)}(t+u) \mathrm{d} u=\sqrt{T} X^{T}\left(\frac{t}{T}\right)+\bar{X}(t)
$$

and hence,

$$
\begin{aligned}
& \Delta_{-\frac{\pi^{2}}{2}, T}=\frac{1}{\sqrt{T}} \int_{0}^{T} X^{T}\left(\frac{t}{T}\right) \mathrm{d} W(t)+I_{1}(T)=\int_{0}^{1} X^{T}(s) \mathrm{d} W^{T}(s)+I_{1}(T), \\
& J_{-\frac{\pi^{2}}{2}, T}=\frac{1}{T} \int_{0}^{T} X^{T}\left(\frac{t}{T}\right)^{2} \mathrm{~d} t+2 I_{2}(T)+I_{3}(T)=\int_{0}^{1} X^{T}(s)^{2} \mathrm{~d} s+2 I_{2}(T)+I_{3}(T),
\end{aligned}
$$

with

$$
I_{1}(T):=\frac{1}{T} \int_{0}^{T} \bar{X}(t) \mathrm{d} W(t), \quad I_{2}(T):=\frac{1}{T^{3 / 2}} \int_{0}^{T} X^{T}\left(\frac{t}{T}\right) \bar{X}(t) \mathrm{d} t, \quad I_{3}(T):=\frac{1}{T^{2}} \int_{0}^{T} \bar{X}(t)^{2} \mathrm{~d} t .
$$

Introducing the process

$$
Y^{T}(t):=\int_{0}^{t} X^{T}(s) \mathrm{d} W^{T}(s), \quad t \in \mathbb{R}_{+}, \quad T \in \mathbb{R}_{++},
$$

we have

$$
\int_{0}^{t} X^{T}(s)^{2} \mathrm{~d} s=\left[Y^{T}, Y^{T}\right](t), \quad t \in \mathbb{R}_{+}, \quad T \in \mathbb{R}_{++},
$$

where $([U, V](t))_{t \in \mathbb{R}_{+}}$denotes the quadratic covariation process of the processes $(U(t))_{t \in \mathbb{R}_{+}}$ and $(V(t))_{t \in \mathbb{R}_{+}}$. Moreover,

$$
Y^{T}(t)=\frac{32 \int_{0}^{t}\left(X_{1}^{T}(s) \mathrm{d} X_{2}^{T}(s)-X_{2}^{T}(s) \mathrm{d} X_{1}^{T}(s)\right)-8 \pi \int_{0}^{t}\left(X_{1}^{T}(s) \mathrm{d} X_{1}^{T}(s)+X_{2}^{T}(s) \mathrm{d} X_{2}^{T}(s)\right)}{\pi\left(\pi^{2}+16\right)}
$$

for $t \in \mathbb{R}_{+}$. By the functional central limit theorem,

$$
\left(X_{1}^{T}, X_{2}^{T}\right) \stackrel{\mathcal{D}}{\longrightarrow} \frac{1}{\sqrt{2}}\left(\mathcal{W}_{1}, \mathcal{W}_{2}\right) \quad \text { as } T \rightarrow \infty
$$

hence

$$
Y^{T} \stackrel{\mathcal{D}}{\longrightarrow} \mathcal{Y} \quad \text { as } T \rightarrow \infty
$$

with

$$
\mathcal{Y}(t):=\frac{16 \int_{0}^{t}\left(\mathcal{W}_{1}(s) \mathrm{d} \mathcal{W}_{2}(s)-\mathcal{W}_{2}(s) \mathrm{d} \mathcal{W}_{1}(s)\right)-4 \pi \int_{0}^{t}\left(\mathcal{W}_{1}(s) \mathrm{d} \mathcal{W}_{1}(s)+\mathcal{W}_{2}(s) \mathrm{d} \mathcal{W}_{2}(s)\right)}{\pi\left(\pi^{2}+16\right)}
$$

for $t \in \mathbb{R}_{+}$. Further, by Corollary 4.12 in Gushchin and Küchler [16],

$$
\left(Y^{T}(1),\left[Y^{T}, Y^{T}\right](1)\right) \stackrel{\mathcal{D}}{\longrightarrow}(\mathcal{Y}(1),[\mathcal{Y}, \mathcal{Y}](1)) \quad \text { as } T \rightarrow \infty
$$


Here we have

$$
\begin{aligned}
{[\mathcal{Y}, \mathcal{Y}](1) } & =\frac{\int_{0}^{1}\left(16 \mathcal{W}_{1}(s)-4 \pi \mathcal{W}_{2}(s)\right)^{2} \mathrm{~d} s+\int_{0}^{1}\left(16 \mathcal{W}_{2}(s)+4 \pi \mathcal{W}_{1}(s)\right)^{2} \mathrm{~d} s}{\pi^{2}\left(\pi^{2}+16\right)^{2}} \\
& =\frac{16}{\pi^{2}\left(\pi^{2}+16\right)} \int_{0}^{1}\left(\mathcal{W}_{1}(s)^{2}+\mathcal{W}_{2}(s)^{2}\right) \mathrm{d} s
\end{aligned}
$$

Recall that $I_{3}(T) \stackrel{\mathbb{P}}{\longrightarrow} 0$ as $T \rightarrow \infty$, which also implies $I_{1}(T) \stackrel{\mathbb{P}}{\longrightarrow} 0$ as $T \rightarrow \infty$. Finally,

$$
\left|I_{2}(T)\right| \leqslant \sqrt{\frac{1}{T^{3}} \int_{0}^{T} X^{T}\left(\frac{t}{T}\right)^{2} \mathrm{~d} t \int_{0}^{T} \bar{X}(t)^{2} \mathrm{~d} t}=\sqrt{\frac{1}{T^{2}} \int_{0}^{1} X^{T}(s)^{2} \mathrm{~d} s \int_{0}^{T} \bar{X}(t)^{2} \mathrm{~d} t} \stackrel{\mathbb{P}}{\longrightarrow} 0
$$

as $T \rightarrow \infty$, and the claim follows.

Proof of Proposition 3.2.7. We have

$$
J_{\vartheta, T}=\mathrm{e}^{-2 v_{0}(\vartheta) T} \int_{0}^{T}\left(\int_{-1}^{0} X^{(\vartheta)}(t+u) \mathrm{d} u\right)^{2} \mathrm{~d} t \quad T \in \mathbb{R}_{+} .
$$

The process $\left(\int_{-1}^{0} X^{(\vartheta)}(t+u) \mathrm{d} u\right)_{t \in[1, \infty)}$ has a representation $(3.2 .4)$ with $y(t)=\int_{-1}^{0} x_{0, \vartheta}(t+$ u) $\mathrm{d} u, \quad t \in \mathbb{R}_{+}$, see the proof of Proposition 3.2.4. The assumption $\vartheta \in(0, \infty)$ implies $v_{0}(\vartheta)>0$ and $v_{1}(\vartheta)<0$, hence by Lemma 3.2.1, there exists $\gamma \in\left(v_{1}(\vartheta), 0\right)$ such that

$$
x_{0, \vartheta}(t)=\frac{v_{0}(\vartheta)}{v_{0}(\vartheta)^{2}+2 v_{0}(\vartheta)-\vartheta} \mathrm{e}^{v_{0}(\vartheta) t}+\mathrm{o}\left(\mathrm{e}^{\gamma t}\right), \quad \text { as } t \rightarrow \infty .
$$

Consequently,

$$
\int_{-1}^{0} x_{0, \vartheta}(t+u) \mathrm{d} u=\frac{1-\mathrm{e}^{-v_{0}(\vartheta)}}{v_{0}(\vartheta)^{2}+2 v_{0}(\vartheta)-\vartheta} \mathrm{e}^{v_{0}(\vartheta) t}+\mathrm{o}\left(\mathrm{e}^{\gamma t}\right), \quad \text { as } t \rightarrow \infty .
$$

Applying Lemma 3.2.12, one can obtain

$$
J_{\vartheta, T} \stackrel{\mathbb{P}}{\longrightarrow} \frac{1}{2 v_{0}(\vartheta)}\left(\frac{1-\mathrm{e}^{-v_{0}(\vartheta)}}{v_{0}(\vartheta)^{2}+2 v_{0}(\vartheta)-\vartheta}\right)^{2}\left(U^{(\vartheta)}\right)^{2}=J_{\vartheta} \quad \text { as } \quad T \rightarrow \infty .
$$

Theorem VIII.5.42 of Jacod and Shiryaev [25] yields the statement.

Proof of Proposition 3.2.8. We have again

$$
J_{\vartheta, T}=\mathrm{e}^{-2 v_{0}(\vartheta) T} \int_{0}^{T}\left(\int_{-1}^{0} X^{(\vartheta)}(t+u) \mathrm{d} u\right)^{2} \mathrm{~d} t \quad T \in \mathbb{R}_{+}
$$

and the process $\left(\int_{-1}^{0} X^{(\vartheta)}(t+u) \mathrm{d} u\right)_{t \in[1, \infty)}$ has a representation $(3.2 .4)$ with $y(t)=\int_{-1}^{0} x_{0, \vartheta}(t+$ u) $\mathrm{d} u, t \in \mathbb{R}_{+}$, see the proof of Proposition 3.2.4. The assumption $\vartheta \in\left(-\infty,-\frac{\pi^{2}}{2}\right)$ implies $v_{0}(\vartheta)>0$ and $v_{0}(\vartheta) \notin \Lambda_{\vartheta}$, hence by Lemma 3.2.1, there exists $\gamma \in\left(0, v_{0}(\vartheta)\right)$ such that

$$
x_{0, \vartheta}(t)=\varphi_{\vartheta}(t) \mathrm{e}^{v_{0}(\vartheta) t}+\mathrm{o}\left(\mathrm{e}^{\gamma t}\right), \quad \text { as } t \rightarrow \infty .
$$

Applying Lemma 3.2.13, one can obtain

$$
J_{\vartheta, T}-J_{\vartheta}(T) \stackrel{\mathbb{P}}{\longrightarrow} 0, \quad \text { as } T \rightarrow \infty .
$$

The process $\left(J_{\vartheta}(t)\right)_{t \in \mathbb{R}_{+}}$is periodic with period $D=\frac{\pi}{\kappa_{0}(\vartheta)}$. 


\subsection{The general case}

Consider now the SDDE (3.1.1)

$$
\begin{cases}\mathrm{d} X(t)=\vartheta \int_{[-r, 0]} X(t+u) a(\mathrm{~d} u) \mathrm{d} t+\mathrm{d} W(t), & t \in \mathbb{R}_{+}, \\ X(t)=X_{0}(t), & t \in[-r, 0],\end{cases}
$$

where now the delay measure $a$ is an arbitrary finite signed measure on $[-r, 0]$. We would like to investigate the local asymptotic properties of this model. As was mentioned in the beginning of the Section 3.2 in this general model it can not be possible to determine the exact values of the parameter $\vartheta$ where the appropriate local asymptotic property is valid. So the aim is to give sufficient conditions for this.

In the uniform delay case and in each of the cited papers, which concern local asymptotic properties of delayed models (Gushchin and Küchler [16], [19]), LAN has been proved in case of $v_{0}(\vartheta)<0$. It turns out that in case of SDDE (3.1.1), LAN holds whenever $v_{\vartheta}^{*}<0$, where $v_{\vartheta}^{*}$ is defined in (3.3.1), see Theorem 3.3.3, but it can happen that $v_{0}(\vartheta)=0$, see the example in Remark 3.3.6. Moreover, LAQ is shown if $v_{\vartheta}^{*}=0$, and, under some additional conditions, LAMN or PLAMN is valid if $v_{\vartheta}^{*}>0$, see Theorems 3.3.4 and 3.3.5. Note that in Theorems 3.3.4 and 3.3.5 we have $v_{\vartheta}^{*}=v_{0}(\vartheta)$, see Remark 3.3.6.

For each $\lambda \in \Lambda_{\vartheta}$, denote by $\widetilde{m}_{\vartheta}(\lambda)$ the degree of the complex-valued polynomial

$$
P_{\vartheta, \lambda}(t):=\sum_{\ell=0}^{m_{\vartheta}(\lambda)-1} c_{\vartheta, \lambda, \ell} t^{\ell}
$$

with

$$
c_{\vartheta, \lambda, \ell}:=\frac{1}{\ell !} \int_{[-r, 0]} \operatorname{Res}_{z=\lambda}\left(\frac{(z-\lambda)^{\ell} \mathrm{e}^{z u}}{h_{\vartheta}(z)}\right) a(\mathrm{~d} u)=\frac{1}{\ell !} \sum_{j=0}^{m_{\vartheta}(\lambda)-1-\ell} \frac{A_{\vartheta,-j-1-\ell}(\lambda)}{j !} \int_{[-r, 0]} u^{j} \mathrm{e}^{\lambda u} a(\mathrm{~d} u),
$$

where the degree of the zero polynomial is defined to be $-\infty$. Put

$$
\begin{aligned}
v_{\vartheta}^{*} & :=\sup \left\{\operatorname{Re}(\lambda): \lambda \in \Lambda_{\vartheta}, \widetilde{m}_{\vartheta}(\lambda) \geqslant 0\right\}, \\
m_{\vartheta}^{*} & :=\max \left\{\widetilde{m}_{\vartheta}(\lambda): \lambda \in \Lambda_{\vartheta}, \operatorname{Re}(\lambda)=v_{\vartheta}^{*}\right\},
\end{aligned}
$$

where $\sup \emptyset:=-\infty$ and $\max \emptyset:=-\infty$.

\subsubsection{Radon-Nikodym derivatives}

From this section, we consider the SDDE (3.1.1) with fixed continuous initial process $\left(X_{0}(t)\right)_{t \in[-r, 0]}$. Further, for all $T \in \mathbb{R}_{++}$, let $\mathbb{P}_{\vartheta, T}$ be the probability measure induced by $\left(X^{(\vartheta)}(t)\right)_{t \in[-r, T]}$ on $(C([-r, T], \mathbb{R}), \mathcal{B}(C([-r, T], \mathbb{R})))$. In order to calculate Radon-Nikodym

derivatives $\frac{d \mathbb{P}_{\theta, T}}{d \mathbb{P}_{\vartheta, T}}$ for certain $\theta, \vartheta \in \mathbb{R}$, we need the following statement, which can be derived from formula (7.139) in Section 7.6.4 of Liptser and Shiryaev [33]. 
3.3.1 Lemma. Let $\theta, \vartheta \in \mathbb{R}$. Then for all $T \in \mathbb{R}_{++}$, the measures $\mathbb{P}_{\theta, T}$ and $\mathbb{P}_{\vartheta, T}$ are absolutely continuous with respect to each other, and

$$
\begin{aligned}
\log \frac{\mathrm{d} \mathbb{P}_{\theta, T}}{d \mathbb{P}_{\vartheta, T}}\left(\left.X^{(\vartheta)}\right|_{[-r, T]}\right) & =(\theta-\vartheta) \int_{0}^{T} Y^{(\vartheta)}(t) \mathrm{d} X^{(\vartheta)}(t)-\frac{1}{2}\left(\theta^{2}-\vartheta^{2}\right) \int_{0}^{T} Y^{(\vartheta)}(t)^{2} \mathrm{~d} t \\
& =(\theta-\vartheta) \int_{0}^{T} Y^{(\vartheta)}(t) \mathrm{d} W(t)-\frac{1}{2}(\theta-\vartheta)^{2} \int_{0}^{T} Y^{(\vartheta)}(t)^{2} \mathrm{~d} t
\end{aligned}
$$

with

$$
Y^{(\vartheta)}(t):=\int_{[-r, 0]} X^{(\vartheta)}(t+u) a(\mathrm{~d} u), \quad t \in \mathbb{R}_{+} .
$$

In order to investigate local asymptotic properties of the family

$$
\left(\mathcal{E}_{T}\right)_{T \in \mathbb{R}_{++}}:=\left(C\left(\mathbb{R}_{+}, \mathbb{R}\right), \mathcal{B}\left(C\left(\mathbb{R}_{+}, \mathbb{R}\right)\right),\left\{\mathbb{P}_{\vartheta, T}: \vartheta \in \mathbb{R}\right\}\right)_{T \in \mathbb{R}_{++}}
$$

of statistical experiments, we derive the following corollary.

3.3.2 Corollary. For each $\vartheta \in \mathbb{R}, T \in \mathbb{R}_{++}, r_{\vartheta, T} \in \mathbb{R}$ and $h_{T} \in \mathbb{R}$, we have

$$
\log \frac{\mathrm{d} \mathbb{P}_{\vartheta+r_{\vartheta, T}} h_{T}, T}{\mathrm{~d} \mathbb{P}_{\vartheta, T}}\left(\left.X^{(\vartheta)}\right|_{[-r, T]}\right)=h_{T} \Delta_{\vartheta, T}-\frac{1}{2} h_{T}^{2} J_{\vartheta, T},
$$

with

$$
\Delta_{\vartheta, T}:=r_{\vartheta, T} \int_{0}^{T} Y^{(\vartheta)}(t) \mathrm{d} W(t), \quad J_{\vartheta, T}:=r_{\vartheta, T}^{2} \int_{0}^{T} Y^{(\vartheta)}(t)^{2} \mathrm{~d} t
$$

\subsubsection{Local asymptotics of the likelihood ratios}

3.3.3 Theorem. If $\vartheta \in \mathbb{R}$ with $v_{\vartheta}^{*}<0$, then the family $\left(\mathcal{E}_{T}\right)_{T \in \mathbb{R}_{++}}$of statistical experiments given in (3.3.3) is LAN at $\vartheta$ with scaling $r_{\vartheta, T}=T^{-1 / 2}, T \in \mathbb{R}_{++}$, and with

$$
J_{\vartheta}=\int_{0}^{\infty}\left(\int_{[-r, 0]} x_{0, \vartheta}(t+u) a(\mathrm{~d} u)\right)^{2} \mathrm{~d} t .
$$

Particularly, if $a([-r, 0])=0$, then $v_{0}^{*}=-\infty, m_{0}^{*}=-\infty$, and the family $\left(\mathcal{E}_{T}\right)_{T \in \mathbb{R}_{++}}$of statistical experiments given in (3.3.3) is LAN at 0 with scaling $r_{0, T}=T^{-1 / 2}, \quad T \in \mathbb{R}_{++}$, and with

$$
J_{0}=\int_{0}^{r} a([-t, 0])^{2} \mathrm{~d} t .
$$

3.3.4 Theorem. If $\vartheta \in \mathbb{R}$ with $v_{\vartheta}^{*}=0$, then the family $\left(\mathcal{E}_{T}\right)_{T \in \mathbb{R}_{++}}$of statistical experiments given in (3.3.3) is LAQ at $\vartheta$ with scaling $r_{\vartheta, T}=T^{-m_{\vartheta}^{*}-1}$ and with

$$
\begin{gathered}
\Delta_{\vartheta}=\sum_{\substack{\lambda \in \Lambda_{\vartheta} \cap(i \mathbb{R}) \\
\tilde{m}_{\vartheta}(\lambda)=m_{\vartheta}^{*}}} c_{\vartheta, \lambda, m_{\vartheta}^{*}} \int_{0}^{1} \mathcal{Z}_{\operatorname{Im}(\lambda), m_{\vartheta}^{*}}(s) \mathrm{d} \overline{\mathcal{Z}_{\operatorname{Im}(\lambda), 0}(s)}, \\
J_{\vartheta}=\sum_{\substack{\lambda \in \Lambda_{\vartheta} \cap(\mathbb{i} \mathbb{R}) \\
\tilde{m}_{\vartheta}(\lambda)=m_{\vartheta}^{*}}}\left|c_{\vartheta, \lambda, m_{\vartheta}^{*}}\right|^{2} \int_{0}^{1}\left|\mathcal{Z}_{\operatorname{Im}(\lambda), m_{\vartheta}^{*}}(s)\right|^{2} \mathrm{~d} s,
\end{gathered}
$$


with

$$
\mathcal{Z}_{\varphi, 0}:= \begin{cases}\mathcal{W}, & \text { if } \varphi=0 \\ \frac{1}{\sqrt{2}}\left(\mathcal{W}_{\varphi, \operatorname{Re}}+\mathrm{i} \mathcal{W}_{\varphi, \operatorname{Im}}\right), & \text { if } \varphi \in \mathbb{R}_{++}, \\ \mathcal{Z}_{-\varphi, 0}, & \text { if } \varphi \in \mathbb{R}_{--},\end{cases}
$$

where $(\mathcal{W}(s))_{s \in[0,1]}, \quad\left(\mathcal{W}_{\varphi, \operatorname{Re}}(s)\right)_{s \in[0,1]}$ and $\left(\mathcal{W}_{\varphi, \operatorname{Im}}(s)\right)_{s \in[0,1]}, \quad \varphi \in \mathbb{R}_{++}$, are independent standard Wiener processes, and

$$
\mathcal{Z}_{\varphi, \ell}(s):=\int_{0}^{s}(s-u)^{\ell} \mathrm{d} \mathcal{Z}_{\varphi, 0}(u), \quad s \in[0,1], \quad \varphi \in \mathbb{R}, \quad \ell \in \mathbb{N} .
$$

Particularly, if $a([-r, 0]) \neq 0$, then $v_{0}^{*}=0, m_{0}^{*}=0$, and the family $\left(\mathcal{E}_{T}\right)_{T \in \mathbb{R}_{++}}$of statistical experiments given in (3.3.3) is $L A Q$ at 0 with scaling $r_{0, T}=T^{-1}, T \in \mathbb{R}_{++}$, and with

$$
\Delta_{0}=a([-r, 0]) \int_{0}^{1} \mathcal{W}(s) \mathrm{d} \mathcal{W}(s), \quad J_{0}=a([-r, 0])^{2} \int_{0}^{1} \mathcal{W}(s)^{2} \mathrm{~d} s .
$$

Note that $\Delta_{\vartheta}$ is real-valued, since

$$
c_{\vartheta, \bar{\lambda}, m_{\vartheta}^{*}} \int_{0}^{1} \mathcal{Z}_{\operatorname{Im}(\bar{\lambda}), m_{\vartheta}^{*}}(s) \mathrm{d} \overline{\mathcal{Z}_{\operatorname{Im}(\bar{\lambda}), 0}(s)}=\overline{c_{\vartheta, \lambda, m_{\vartheta}^{*}}} \int_{0}^{1} \overline{\mathcal{Z}_{\operatorname{Im}(\lambda), m_{\vartheta}^{*}}(s)} \mathrm{d} \mathcal{Z}_{\operatorname{Im}(\lambda), 0}(s), \quad \lambda \in \Lambda_{\vartheta} .
$$

3.3.5 Theorem. Let $\vartheta \in \mathbb{R}$ with $v_{\vartheta}^{*}>0$. If

$$
H_{\vartheta}:=\left\{\operatorname{Im}(\lambda): \lambda \in \Lambda_{\vartheta} \cap\left(v_{\vartheta}^{*}+i \mathbb{R}_{++}\right), \widetilde{m}_{\vartheta}(\lambda)=m_{\vartheta}^{*}\right\} \neq \emptyset
$$

and the numbers in $H_{\vartheta}$ have a common divisor $D_{\vartheta}$ (namely, they are pairwise commensurable, and the quotients of these numbers and $D_{\vartheta}$ are integers), then the family $\left(\mathcal{E}_{T}\right)_{T \in \mathbb{R}_{++}}$of statistical experiments given in (3.3.3) is PLAMN at $\vartheta$ with period $\frac{2 \pi}{D_{\vartheta}}$, with scaling $r_{\vartheta, T}=$ $T^{-m_{\vartheta}^{*}} \mathrm{e}^{-v_{\vartheta}^{*} T}, T \in \mathbb{R}_{++}$, and with

$$
\Delta_{\vartheta}(d)=Z \sqrt{J_{\vartheta}(d)}, \quad J_{\vartheta}(d)=\int_{0}^{\infty} \mathrm{e}^{-2 v_{\vartheta}^{*} t} \operatorname{Re}\left(\sum_{\substack{\lambda \in \Lambda_{\vartheta} \cap\left(v_{\vartheta}^{*}+\mathrm{i} \mathbb{R}\right) \\ \widetilde{m}_{\vartheta}(\lambda)=m_{\vartheta}^{*}}} c_{\vartheta, \lambda, m_{\vartheta}^{*}} U_{\lambda}^{(\vartheta)} \mathrm{e}^{\mathrm{i}(d-t) \operatorname{Im}(\lambda)}\right)^{2} \mathrm{~d} t
$$

for $d \in\left[0, \frac{2 \pi}{D_{\vartheta}}\right)$, where

$$
U_{\lambda}^{(\vartheta)}:=X_{0}(0)+v_{\vartheta}^{*} \int_{[-r, 0]} \int_{u}^{0} \mathrm{e}^{-\lambda(s-u)} X_{0}(s) \mathrm{d} s a(\mathrm{~d} u)+\int_{0}^{\infty} \mathrm{e}^{-\lambda s} \mathrm{~d} W(s), \quad \lambda \in \mathbb{C},
$$

and $Z$ is a standard normally distributed random variable independent of $\left(X_{0}(t)\right)_{t \in[-r, 0]}$ and $(W(t))_{t \in \mathbb{R}_{+}}$.

If $H_{\vartheta}=\emptyset$, then the family $\left(\mathcal{E}_{T}\right)_{T \in \mathbb{R}_{++}}$of statistical experiments given in (3.3.3) is $L A M N$

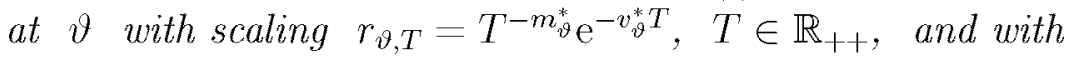

$$
\Delta_{\vartheta}=Z \sqrt{J_{\vartheta}}, \quad J_{\vartheta}=\frac{c_{\vartheta, v_{\vartheta}^{*}, m_{\vartheta}^{*}}^{2}}{2 v_{\vartheta}^{*}}\left(U_{v_{\vartheta}^{*}}^{(\vartheta)}\right)^{2} .
$$


The Theorem 3.3.5 is not complete in the sense, that we have not covered the case when the set $H_{\vartheta}$ is not the empty set, but the elements in it have not a common divisor, namely they are incommensurable.

3.3.6 Remark. According to the definition of $v_{0}(\vartheta)$ and $v_{\vartheta}^{*}$, one can obtain $v_{0}(\vartheta) \geq v_{\vartheta}^{*}$. The aim of the following discussion is to show that $v_{0}(\vartheta)>v_{\vartheta}^{*}$ if and only if $\left\{\lambda \in \Lambda_{\vartheta}\right.$ : $\operatorname{Re}(\lambda) \geqslant 0\}=\{0\}$ and $P_{\vartheta, 0}=0$ (and hence $v_{0}(\vartheta)=0$ and $v_{\vartheta}^{*}<0$ ). Indeed, if $v_{0}(\vartheta)>v_{\vartheta}^{*}$ then for all $\lambda_{0} \in \Lambda_{\vartheta}$ with $\operatorname{Re}\left(\lambda_{0}\right)>v_{\vartheta}^{*}$ we have $P_{\vartheta, \lambda_{0}}=0$, implying

$$
c_{\vartheta, \lambda_{0}, m_{\vartheta}\left(\lambda_{0}\right)-1}=\frac{1}{\left(m_{\vartheta}\left(\lambda_{0}\right)-1\right) !} A_{\vartheta,-m_{\vartheta}\left(\lambda_{0}\right)}\left(\lambda_{0}\right) \int_{[-r, 0]} \mathrm{e}^{\lambda_{0} u} a(\mathrm{~d} u)=0 .
$$

Here $A_{\vartheta,-m_{\vartheta}\left(\lambda_{0}\right)}(\lambda) \neq 0$, since it is the leading coefficient of the polynomial $p_{\vartheta, \lambda_{0}}$ of degree $m_{\vartheta}\left(\lambda_{0}\right)-1$, hence $c_{\vartheta, \lambda_{0}, m_{\vartheta}\left(\lambda_{0}\right)-1}=0$ yields $\int_{[-r, 0]} e^{\lambda_{0} u} a(\mathrm{~d} u)=0$. Taking into account of the characteristic equation, one can get $\lambda_{0}=0$, hence $\left\{\lambda \in \Lambda_{\vartheta}: \operatorname{Re}(\lambda)>v_{\vartheta}^{*}\right\}=\{0\}$. Clearly, this yields also $v_{0}(\vartheta)=0$ and $v_{\vartheta}^{*}<0$, and hence $\left\{\lambda \in \Lambda_{\vartheta}: \operatorname{Re}(\lambda) \geqslant 0\right\}=\{0\}$. Conversely, if $\left\{\lambda \in \Lambda_{\vartheta}: \operatorname{Re}(\lambda) \geqslant 0\right\}=\{0\}$ and $P_{\vartheta, 0}=0$, then, by definition, $v_{0}(\vartheta)=0$ and $v_{\vartheta}^{*}<0$.

In particular, if $\left\{\lambda \in \Lambda_{\vartheta}: \operatorname{Re}(\lambda) \geqslant 0\right\}=\{0\}$ and $m_{\vartheta}(0)=1$, then $v_{0}(\vartheta)>v_{\vartheta}^{*}$ is equivalent to $a([-r, 0])=0$, since $P_{\vartheta, 0}=c_{\vartheta, 0,0}=\int_{[-r, 0]} \mathrm{e}^{\lambda_{0} u} a(\mathrm{~d} u)=a([-r, 0])$.

An example for this situation is, when $r=2 \pi, a(\mathrm{~d} u)=\sin (u) \mathrm{d} u$ and $\vartheta \in\left(0, \frac{1}{\pi}\right)$, see Example 3.3.7.

3.3.7 Example. In this example we investigate the special case, when $r=2 \pi$ and $a(\mathrm{~d} u)=$ $\sin (u) \mathrm{d} u$.

The following results can be derived by applying usual methods (e.g., argument principle in complex analysis and the existence of local inverses of holomorphic functions), see, e.g., Reifs $[38$, Section 2.4].

In the trivial case of $\vartheta=0$ the LAN property holds due to the fact that $a([-2 \pi, 0])=0$, see Theorem 3.3.3. In the sequel, we suppose $\vartheta \neq 0$. The characteristic function has the form

$$
h_{\vartheta}(\lambda)=\lambda-\vartheta \int_{-2 \pi}^{0} \mathrm{e}^{\lambda u} \sin (u) \mathrm{d} u=\left\{\begin{array}{ll}
\frac{\lambda^{3}+\lambda-\left(\mathrm{e}^{-2 \pi \lambda}-1\right) \vartheta}{\lambda^{2}+1}, & \text { if } \lambda \neq \pm \mathrm{i} \\
\lambda \mp \vartheta \pi \mathrm{i}, & \text { if } \lambda= \pm \mathrm{i} .
\end{array} .\right.
$$

Thus $0 \in \Lambda_{\vartheta}$, and hence $v_{0}(\vartheta) \geqslant 0$ for all $\vartheta \in \mathbb{R}$. Moreover, $\pm i \in \Lambda_{\vartheta}$ if and only if $\vartheta=\frac{1}{\pi}$. Any purely imaginary zero $\lambda=\mathrm{i} y$ with $y \in \mathbb{R} \backslash\{ \pm 1\}$ of $h_{\vartheta}$ satisfies the system of equations

$$
\left\{\begin{array}{l}
(\cos (2 \pi y)-1) \vartheta=0 \\
-y^{3}+y+\vartheta \sin (2 \pi y)=0
\end{array}\right.
$$

The first equation and $\vartheta \neq 0$ yield $y=k, k \in \mathbb{Z}$. The second equation implies $k^{3}-k=0$, hence $y \neq \pm 1$ yields $k=0$. Thinking of the parameter $\vartheta=\vartheta(\lambda)$ to be dependent on the zero $\lambda$ of $h_{\vartheta}$ and allowing for complex values of $\vartheta$, we have

$$
\vartheta(\lambda)=\frac{\lambda^{3}+\lambda}{\mathrm{e}^{-2 \pi \lambda}-1}
$$


for $\lambda \in \mathbb{C}$ with $\operatorname{Re}(\lambda) \geqslant 0$ and $\operatorname{Im}(\lambda) \notin \mathbb{Z}$. We have $\lim _{\lambda \rightarrow 0} \vartheta(\lambda)=-\frac{1}{2 \pi}$ and $\lim _{\lambda \rightarrow \pm \mathrm{i}} \vartheta(\lambda)=$ $\frac{1}{\pi}$, hence the number of zeros of $h_{\vartheta}$ is constant as a function of $\vartheta$ on each of the open intervals $\left(-\infty,-\frac{1}{2 \pi}\right),\left(-\frac{1}{2 \pi}, \frac{1}{\pi}\right)$ and $\left(\frac{1}{\pi}, \infty\right)$, see, e.g., Reiß [38, Lemma 3]. Further, we have

$$
\vartheta^{\prime}(\lambda)=\frac{\left(3 \lambda^{2}+1\right)\left(\mathrm{e}^{-2 \pi \lambda}-1\right)+2 \pi\left(\lambda^{3}+\lambda\right) \mathrm{e}^{-2 \pi \lambda}}{\left(\mathrm{e}^{-2 \pi \lambda}-1\right)^{2}}
$$

for $\lambda \in \mathbb{C}$ with $\operatorname{Re}(\lambda) \geqslant 0$ and $\operatorname{Im}(\lambda) \notin \mathbb{Z}$. Applying $\mathrm{e}^{-2 \pi y}=1-2 \pi y+2 \pi^{2} y^{2}+\mathrm{O}\left(y^{3}\right)$ and $\mathrm{e}^{-2 \pi y \mathrm{i}}=\mathrm{e}^{-2 \pi(y-1) \mathrm{i}}=1-2 \pi(y-1) \mathrm{i}-2 \pi^{2}(y-1)^{2}+\mathrm{O}\left((y-1)^{3}\right)$, one can obtain $\lim _{\lambda \rightarrow 0} \vartheta^{\prime}(\lambda)=-\frac{1}{2}$ and $\lim _{\lambda \rightarrow \mathrm{i}} \vartheta(\lambda)=1+\frac{3}{2 \pi} \mathrm{i}$. By the existence of local inverses of holomorphic functions, we can define the inverse $\lambda(\vartheta)$ of $\vartheta(\lambda)$ locally around $\vartheta\left(-\frac{1}{2 \pi}\right)$ and $\vartheta\left(\frac{1}{\pi}\right)$, and its derivative at $-\frac{1}{2 \pi}$ and $\frac{1}{\pi}$ are

$$
\lambda^{\prime}\left(-\frac{1}{2 \pi}\right)=\frac{1}{\vartheta^{\prime}(0)}=-2, \quad \lambda^{\prime}\left(\frac{1}{\pi}\right)=\frac{1}{\vartheta^{\prime}(\mathrm{i})}=\frac{1-\frac{3}{2 \pi}}{\left|1+\frac{3}{2 \pi}\right|^{2}} .
$$

Hence $\operatorname{Re}\left(\lambda^{\prime}\left(-\frac{1}{2 \pi}\right)\right)<0$ and $\operatorname{Re}\left(\lambda^{\prime}\left(\frac{1}{\pi}\right)\right)>0$. Consequently, locally at $-\frac{1}{2 \pi}$, at least one zeros of $h_{\vartheta}$ cross the imaginary axis from the right to the left, and locally at $\frac{1}{\pi}$, at least one zeros of $h_{\vartheta}$ cross the imaginary axis from the left to the right as $\vartheta$ increases. Not more than one real zero moves into the left half plane locally at $-\frac{1}{2 \pi}$, and not more than two zeros move into the right half plane locally at $\frac{1}{\pi}$, see, e.g., Reiß $[38,2.4]$. Thus, we have the following cases:

(i) If $\vartheta<-\frac{1}{2 \pi}$, then $v_{0}(\vartheta)>0, v_{0}(\vartheta) \in \Lambda_{\vartheta}$ and $m_{\vartheta}\left(v_{0}(\vartheta)\right)=1$.

(ii) If $\vartheta=-\frac{1}{2 \pi}$, then $v_{0}\left(-\frac{1}{2 \pi}\right)=0 \in \Lambda_{-\frac{1}{2 \pi}}$ and $\left.m_{-\frac{1}{2 \pi}}(0)\right)=2$.

(iii) If $\vartheta \in\left(-\frac{1}{2 \pi}, \frac{1}{\pi}\right)$, then $v_{0}(\vartheta)=0 \in \Lambda_{\vartheta}$ and $m_{\vartheta}(0)=1$.

(iv) If $\vartheta=\frac{1}{\pi}$, then $v_{0}\left(\frac{1}{\pi}\right)=0,0, \pm \mathrm{i} \in \Lambda_{\frac{1}{\pi}}$ and $m_{\frac{1}{\pi}}(0)=m_{\frac{1}{\pi}}( \pm \mathrm{i})=1$.

(v) If $\vartheta>\frac{1}{\pi}$, then $v_{0}(\vartheta)>0, v_{0}(\vartheta) \notin \Lambda_{\vartheta}$ and $m_{\vartheta}\left(v_{0}(\vartheta)\right)=1$.

Finally, we have to calculate $\widetilde{m}_{\vartheta}(\lambda)$ for some specific $\lambda \in \Lambda_{\vartheta}$ to derive the $\operatorname{sign}$ of $v_{\vartheta}^{*}$. Namely, if $\vartheta \neq-\frac{1}{2 \pi}$, then we have

$$
P_{\vartheta, 0}(t)=c_{\vartheta, 0,0}=A_{\vartheta,-1}(0) \int_{-2 \pi}^{0} \sin (u) \mathrm{d} u=0
$$

hence $\widetilde{m}_{\vartheta}(0)=-\infty$. Furthermore, if $\vartheta=-\frac{1}{2 \pi}$, then we have

$$
\begin{aligned}
& P_{-\frac{1}{2 \pi}, 0}(t)=c_{-\frac{1}{2 \pi}, 0,0}+c_{-\frac{1}{2 \pi}, 0,1} t \\
& =A_{-\frac{1}{2 \pi},-1}(0) \int_{-2 \pi}^{0} \sin (u) \mathrm{d} u+A_{-\frac{1}{2 \pi},-2}(0) \int_{-2 \pi}^{0} u \sin (u) \mathrm{d} u+A_{-\frac{1}{2 \pi},-2}(0) t \int_{-2 \pi}^{0} \sin (u) \mathrm{d} u \\
& =-2 \pi A_{-\frac{1}{2 \pi},-2}(0) \neq 0,
\end{aligned}
$$

hence $\widetilde{m}_{-\frac{1}{2 \pi}}(0)=0$. In the other cases the leading coefficient $c_{\vartheta, \lambda, m_{\vartheta}}(\lambda)-1$ does not vanish, thus $\widetilde{m}_{\vartheta}(\lambda)=m_{\vartheta}(\lambda)-1$, consequently, we conclude the following final results: 
(i) If $\vartheta<-\frac{1}{2 \pi}$, then $v_{\vartheta}^{*}=v_{0}(\vartheta)>0, m_{\vartheta}^{*}=0$ and $H_{\vartheta}=\emptyset$, hence the LAMN property holds with scaling $\mathrm{e}^{-v_{0}(\vartheta) T}, T \in \mathbb{R}_{++}$.

(ii) If $\vartheta=-\frac{1}{2 \pi}$, then $v_{-\frac{1}{2 \pi}}^{*}=v_{0}\left(-\frac{1}{2 \pi}\right)=0$ and $m_{-} \frac{1}{2 \pi}^{*}=0$, hence the LAQ property holds with scaling $T^{-1}, T \in \mathbb{R}_{++}$.

(iii) If $\vartheta \in\left(-\frac{1}{2 \pi}, \frac{1}{\pi}\right)$, then $v_{\vartheta}^{*}<0$, hence the LAN property holds with scaling $T^{-1 / 2}$, $T \in \mathbb{R}_{++}$, although $v_{0}(\vartheta)=0$.

(iv) If $\vartheta=\frac{1}{\pi}$, then $v_{\frac{1}{\tau}}^{*}=v_{0}\left(\frac{1}{\pi}\right)=0$ and $m_{\frac{1}{\pi}}^{*}=0$, hence the LAQ property holds with scaling $T^{-1}, T \in \mathbb{R}_{++}$.

(v) If $\vartheta>\frac{1}{\pi}$, then $v_{\vartheta}^{*}>0, m_{\vartheta}^{*}=0$ and $H_{\vartheta}=\left\{\kappa_{0}(\vartheta)\right\}$, where $\kappa_{0}(\vartheta)=\left|\operatorname{Im}\left(\lambda_{0}(\vartheta)\right)\right|$ is given by $\operatorname{Re}\left(\lambda_{0}(\vartheta)\right)=v_{\vartheta}^{*}$. Hence the PLAMN property holds with period $\frac{2 \pi}{\kappa_{0}(\vartheta)}$ and with scaling $\mathrm{e}^{-v_{0}(\vartheta) T}, T \in \mathbb{R}_{++}$.

\subsubsection{Maximum likelihood estimates}

For fixed $T \in \mathbb{R}_{++}$, maximizing $\log \frac{\mathrm{dP}_{\vartheta, T}}{\mathrm{dP}_{0, T}}\left(\left.X^{(\vartheta)}\right|_{[-r, T]}\right)$ in $\vartheta \in \mathbb{R}$ gives the MLE of $\vartheta$ based on the observations $(X(t))_{t \in[-r, T]}$ having the form

$$
\widehat{\vartheta}_{T}=\frac{\int_{0}^{T} \int_{[-r, 0]} X^{(\vartheta)}(t+u) a(\mathrm{~d} u) \mathrm{d} X^{(\vartheta)}(t)}{\int_{0}^{T}\left(\int_{[-r, 0]} X^{(\vartheta)}(t+u) a(\mathrm{~d} u)\right)^{2} \mathrm{~d} t}
$$

provided that $\int_{0}^{T}\left(\int_{[-r, 0]} X^{(\vartheta)}(t+u) a(\mathrm{~d} u)\right)^{2} \mathrm{~d} t>0$. Using the SDDE (3.1.1), one can check that

$$
\widehat{\vartheta}_{T}-\vartheta=\frac{\int_{0}^{T} \int_{[-r, 0]} X^{(\vartheta)}(t+u) a(\mathrm{~d} u) \mathrm{d} W(t)}{\int_{0}^{T}\left(\int_{[-r, 0]} X^{(\vartheta)}(t+u) a(\mathrm{~d} u)\right)^{2} \mathrm{~d} t},
$$

hence

$$
r_{\vartheta, T}^{-1}\left(\widehat{\vartheta}_{T}-\vartheta\right)=\frac{\Delta_{\vartheta, T}}{J_{\vartheta, T}}
$$

Using the results of Section 3.3 .2 and the continuous mapping theorem, we obtain the following result.

3.3.8 Proposition. If $v_{\vartheta}^{*}<0$ then

$$
\sqrt{T}\left(\widehat{\vartheta}_{T}-\vartheta\right) \stackrel{\mathcal{D}}{\longrightarrow} \mathcal{N}\left(0, J_{\vartheta}^{-1}\right) \quad \text { as } T \rightarrow \infty,
$$

where $J_{\vartheta}$ is given in Proposition 3.3.3.

If $v_{\vartheta}^{*}=0$ then

$$
T^{m_{\vartheta}^{*}+1}\left(\widehat{\vartheta}_{T}-\vartheta\right) \stackrel{\mathcal{D}}{\longrightarrow} \frac{\Delta_{\vartheta}}{J_{\vartheta}} \quad \text { as } \quad T \rightarrow \infty
$$


where $\Delta_{\vartheta}$ and $J_{\vartheta}$ is given in Proposition 3.3.4.

If $v_{\vartheta}^{*}>0$ and $H_{\vartheta} \neq \emptyset$ and the numbers in $H_{\vartheta}$ have a common divisor $D_{\vartheta}$, then for each $d \in\left[0, D_{\vartheta}\right)$,

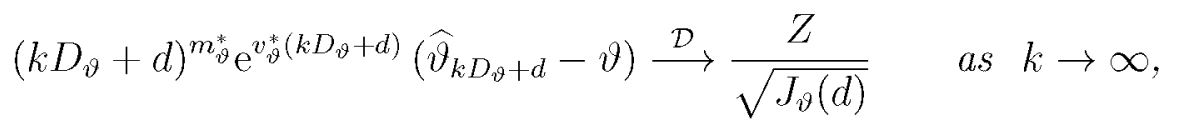

where $J_{\vartheta}(d)$ is given in Proposition 3.3.5, and $Z$ is a standard normally distributed random variable independent of $J_{\vartheta}(d)$.

If $v_{\vartheta}^{*}>0$ and $H_{\vartheta}=\emptyset$ then

$$
T^{m_{\vartheta}^{*}} \mathrm{e}^{v_{\vartheta}^{*} T}\left(\widehat{\vartheta}_{T}-\vartheta\right) \stackrel{\mathcal{D}}{\longrightarrow} \frac{Z}{\sqrt{J_{\vartheta}}} \quad \text { as } T \rightarrow \infty,
$$

where $J_{\vartheta}$ is given in Proposition 3.3.5, and $Z$ is a standard normally distributed random variable independent of $J_{\vartheta}$.

\subsubsection{Proofs}

For each $\vartheta \in \mathbb{R}$ and each $t \in[r, \infty)$, by (3.1.5), we have

$$
\begin{aligned}
Y^{(\vartheta)}(t)= & X_{0}(0) \int_{[-r, 0]} x_{0, \vartheta}(t+u) \mathrm{d} u+\int_{[-r, 0]} \int_{[0, t+u]} W(t+u-s) \mathrm{d} x_{0, \vartheta}(s) a(\mathrm{~d} u) \\
& +\vartheta \int_{[-r, 0]} \int_{[-r, 0]} \int_{v}^{0} x_{0, \vartheta}(t+u+v-s) X_{0}(s) \mathrm{d} s a(\mathrm{~d} v) a(\mathrm{~d} u) .
\end{aligned}
$$

Here we have

$$
\begin{aligned}
\int_{[-r, 0]} \int_{[-r, 0]} & \int_{v}^{0} x_{0, \vartheta}(t+u+v-s) X_{0}(s) \mathrm{d} s a(\mathrm{~d} v) a(\mathrm{~d} u) \\
= & \int_{[-r, 0]} \int_{[-r, 0]} \int_{v}^{0} x_{0, \vartheta}(t+u+v-s) X_{0}(s) \mathrm{d} s a(\mathrm{~d} u) a(\mathrm{~d} v) \\
= & \int_{[-r, 0]} \int_{v}^{0} X_{0}(s) \int_{[-r, 0]} x_{0, \vartheta}(t+u+v-s) a(\mathrm{~d} u) \mathrm{d} s a(\mathrm{~d} v)
\end{aligned}
$$

and

$$
\begin{aligned}
& \int_{[-r, 0]} \int_{0}^{t+u} x_{0, \vartheta}(t+u-s) \mathrm{d} W(s) a(\mathrm{~d} u) \\
& =\int_{0}^{t-r} \int_{[-r, 0]} x_{0, \vartheta}(t+u-s) a(\mathrm{~d} u) \mathrm{d} W(s)+\int_{t-r}^{t} \int_{[s-t, 0]} x_{0, \vartheta}(t+u-s) a(\mathrm{~d} u) \mathrm{d} W(s) \\
& =\int_{0}^{t} \int_{[-r, 0]} x_{0, \vartheta}(t+u-s) a(\mathrm{~d} u) \mathrm{d} W(s),
\end{aligned}
$$


since $t \in[r, \infty), s \in[t-r, t]$ and $u \in[-r, s-t)$ imply $t+u-s \in[-r, 0)$, and hence $x_{0, \vartheta}(t+u-s)=0$. Consequently, the process $\left(Y^{(\vartheta)}(t)\right)_{t \in[r, \infty)}$ has a representation

$$
Y^{(\vartheta)}(t)=y_{\vartheta}(t) X_{0}(0)+\vartheta \int_{[-r, 0]} \int_{v}^{0} y_{\vartheta}(t+v-s) X_{0}(s) \mathrm{d} s a(\mathrm{~d} v)+\int_{0}^{t} y_{\vartheta}(t-s) \mathrm{d} W(s)
$$

for $t \in[r, \infty)$ with

$$
y_{\vartheta}(t):=\int_{[-r, 0]} x_{0, \vartheta}(t+u) a(\mathrm{~d} u), \quad t \in \mathbb{R}_{+} .
$$

Applying Lemma 3.1.1, one can obtain

$$
y_{\vartheta}(t)=\sum_{\substack{\lambda \in \Lambda_{\vartheta} \\ \operatorname{Re}(\lambda) \geqslant c}} \int_{[-r, 0]} \operatorname{Res}_{z=\lambda}\left(\frac{\mathrm{e}^{z(t+u)}}{h_{\vartheta}(z)}\right) a(\mathrm{~d} u)+\int_{[-r, 0]} \psi_{\vartheta, c}(t+u) a(\mathrm{~d} u) .
$$

Here we have

$$
\int_{[-r, 0]} \psi_{\vartheta, c}(t+u) a(\mathrm{~d} u)=\mathrm{o}\left(\mathrm{e}^{c t}\right) \quad \text { as } t \rightarrow \infty
$$

Indeed,

$$
\lim _{t \rightarrow \infty} \mathrm{e}^{-c t} \int_{[-r, 0]} \psi_{\vartheta, c}(t+u) a(\mathrm{~d} u)=\lim _{t \rightarrow \infty} \int_{[-r, 0]}\left[\mathrm{e}^{-c(t+u)} \psi_{\vartheta, c}(t+u)\right] \mathrm{e}^{c u} a(\mathrm{~d} u)=0,
$$

since $a$ is a finite signed measure on $[-r, 0]$. Moreover,

$$
\begin{aligned}
\operatorname{Res}_{z=\lambda}\left(\frac{\mathrm{e}^{z(t+u)}}{h_{\vartheta}(z)}\right) & =\mathrm{e}^{\lambda(t+u)} \sum_{k=-m_{\vartheta}(\lambda)}^{-1} \frac{A_{\vartheta, k}(\lambda)}{(-1-k) !}(t+u)^{-1-k} \\
& =\mathrm{e}^{\lambda(t+u)} \sum_{k=-m_{\vartheta}(\lambda)}^{-1} A_{\vartheta, k}(\lambda) \sum_{\ell=0}^{-1-k} \frac{t^{\ell} u^{-1-k-\ell}}{\ell !(-1-k-\ell) !} \\
& =\mathrm{e}^{\lambda(t+u)} \sum_{\ell=0}^{m_{\vartheta}(\lambda)-1} \frac{t^{\ell}}{\ell !} \sum_{k=-m_{\vartheta}(\lambda)}^{-1-\ell} \frac{A_{\vartheta, k}(\lambda)}{(-1-k-\ell) !} u^{-1-k-\ell} \\
& =\mathrm{e}^{\lambda t} \sum_{\ell=0}^{m_{\vartheta}(\lambda)-1} \frac{t^{\ell}}{\ell !} \operatorname{Res}\left(\frac{(z-\lambda)^{\ell} \mathrm{e}^{z u}}{h_{\vartheta}(z)}\right) .
\end{aligned}
$$

Consequently, one can obtain for each $\vartheta \in \mathbb{R}$ and each $c \in \mathbb{R}$, the representation

$$
y_{\vartheta}(t)=\sum_{\substack{\lambda \in \Lambda_{\vartheta} \\ \operatorname{Re}(\lambda) \geqslant c}} P_{\vartheta, \lambda}(t) \mathrm{e}^{\lambda t}+\Psi_{\vartheta, c}(t) \quad \text { as } t \rightarrow \infty
$$

where $\Psi_{\vartheta, c}: \mathbb{R}_{+} \rightarrow \mathbb{R}$ is a continuous function with $\Psi_{\vartheta, c}(t)=o\left(e^{c t}\right)$ as $t \rightarrow \infty$. Hence we need to analyse the asymptotic behaviour of the right hand side of (3.3.4) as $T \rightarrow \infty$, replacing $y_{\vartheta}(t)$ by $P_{\vartheta, \lambda}(t) \mathrm{e}^{\lambda t}$.

First we derive a good estimate for the second term of the right hand side of (3.3.4). 
3.3.9 Lemma. Let $(y(t))_{t \in \mathbb{R}_{+}}$be a continuous deterministic function. Let a be a signed measure on $[-r, 0]$. Put

$$
I(t):=\int_{[-r, 0]} \int_{u}^{0} y(t+u-s) X_{0}(s) \mathrm{d} s a(\mathrm{~d} u), \quad t \in[r, \infty) .
$$

Then for each $T \in[r, \infty)$,

$$
\begin{gathered}
I(T)^{2} \leqslant|a|([-r, 0]) \int_{-r}^{0} X_{0}(s)^{2} \mathrm{~d} s \int_{0}^{T} y(v)^{2} \mathrm{~d} v \leqslant\|a\| \int_{-r}^{0} X_{0}(s)^{2} \mathrm{~d} s \int_{0}^{T} y(v)^{2} \mathrm{~d} v \\
\int_{r}^{T} I(t)^{2} \mathrm{~d} t
\end{gathered}
$$

where $|a|$ and $\|a\|:=|a|([-r, 0])$ denotes the variation and the total variation of the signed measure a, respectively.

Proof. For each $t \in[r, \infty)$, by Fubini's theorem,

$$
I(t)=\int_{-r}^{0} X_{0}(s) \int_{[-r, s]} y(t+u-s) a(\mathrm{~d} u) \mathrm{d} s .
$$

By the Cauchy-Schwarz inequality,

$$
\begin{aligned}
I(t)^{2} & \leqslant \int_{-r}^{0} X_{0}(s)^{2} \mathrm{~d} s \int_{-r}^{0}\left(\int_{[-r, s]} y(t+u-s) a(\mathrm{~d} u)\right)^{2} \mathrm{~d} s \\
& \leqslant \int_{-r}^{0} X_{0}(s)^{2} \mathrm{~d} s \int_{-r}^{0} \int_{[-r, s]} y(t+u-s)^{2}|a|(\mathrm{d} u) \mathrm{d} s,
\end{aligned}
$$

where, by Fubini's theorem,

$$
\begin{aligned}
\int_{-r}^{0} \int_{[-r, s]} y(t+u-s)^{2}|a|(\mathrm{d} u) \mathrm{d} s & =\int_{[-r, 0]} \int_{u}^{0} y(t+u-s)^{2} \mathrm{~d} s|a|(\mathrm{d} u) \\
& =\int_{[-r, 0]} \int_{t+u}^{t} y(v)^{2} \mathrm{~d} v|a|(\mathrm{d} u) \leqslant \int_{[-r, 0]}|a|(\mathrm{d} u) \int_{0}^{t} y(v)^{2} \mathrm{~d} v
\end{aligned}
$$

hence we obtain (3.3.6). Moreover,

$$
\int_{r}^{T} I(t)^{2} \mathrm{~d} t \leqslant \int_{-r}^{0} X_{0}(s)^{2} \mathrm{~d} s \int_{r}^{T} \int_{-r}^{0} \int_{[-r, s]} y(t+u-s)^{2}|a|(\mathrm{d} u) \mathrm{d} s \mathrm{~d} t
$$


where, by Fubini's theorem,

$$
\begin{array}{r}
\int_{r}^{T} \int_{-r}^{0} \int_{[-r, s]} y(t+u-s)^{2}|a|(\mathrm{d} u) \mathrm{d} s \mathrm{~d} t=\int_{-r}^{0} \int_{[-r, s]} \int_{r}^{T} y(t+u-s)^{2} \mathrm{~d} t|a|(\mathrm{d} u) \mathrm{d} s \\
=\int_{-r}^{0} \int_{[-r, s]} \int_{r+u-s}^{T+u-s} y(v)^{2} \mathrm{~d} v|a|(\mathrm{d} u) \mathrm{d} s \leqslant \int_{0}^{T} y(v)^{2} \mathrm{~d} v \int_{-r}^{0} \int_{[-r, s]}|a|(\mathrm{d} u) \mathrm{d} s \\
=\int_{0}^{T} y(v)^{2} \mathrm{~d} v \int_{[-r, 0]} \int_{u}^{0} \mathrm{~d} s|a|(\mathrm{d} u)=\int_{0}^{T} y(v)^{2} \mathrm{~d} v \int_{[-r, 0]}(-u)|a|(\mathrm{d} u),
\end{array}
$$

hence we obtain $(3.3 .7)$.

3.3.10 Lemma. Let $(y(t))_{t \in \mathbb{R}_{+}}$be a continuous deterministic function with $\int_{0}^{\infty} y(t)^{2} \mathrm{~d} t<\infty$. Let $\vartheta \in \mathbb{R}$. Suppose that $(Y(t))_{t \in \mathbb{R}_{+}}$is a continuous stochastic process such that

$$
Y(t)=y(t) X_{0}(0)+\vartheta \int_{[-r, 0]} \int_{v}^{0} y(t+v-s) X_{0}(s) \mathrm{d} s a(\mathrm{~d} v)+\int_{0}^{t} y(t-s) \mathrm{d} W(s)
$$

for $t \in[r, \infty)$. Then

$$
\begin{gathered}
\frac{1}{T} \int_{0}^{T} Y(t) \mathrm{d} t \stackrel{\mathbb{P}}{\longrightarrow} 0 \quad \text { as } T \rightarrow \infty, \\
\frac{1}{T} \int_{0}^{T} Y(t)^{2} \mathrm{~d} t \stackrel{\mathbb{P}}{\longrightarrow} \int_{0}^{\infty} y(t)^{2} \mathrm{~d} t \quad \text { as } T \rightarrow \infty .
\end{gathered}
$$

Proof. Applying Lemma 4.3 in Gushchin and Küchler [16] for the special case $X_{0}(s)=0$, $s \in[-1,0]$, we obtain

$$
\begin{gathered}
\frac{1}{T} \int_{0}^{T} \int_{0}^{t} y(t-s) \mathrm{d} W(s) \mathrm{d} t \stackrel{\mathbb{P}}{\longrightarrow} 0 \quad \text { as } T \rightarrow \infty \\
\frac{1}{T} \int_{0}^{T}\left(\int_{0}^{t} y(t-s) \mathrm{d} W(s)\right)^{2} \mathrm{~d} t \stackrel{\mathbb{P}}{\longrightarrow} \int_{0}^{\infty} y(t)^{2} \mathrm{~d} t \quad \text { as } T \rightarrow \infty
\end{gathered}
$$

We have

$$
\frac{1}{T} \int_{0}^{T} Y(t) \mathrm{d} t=\frac{1}{T} \int_{0}^{r} Y(t) \mathrm{d} t+X_{0}(0) I_{1}(T)+\frac{\vartheta}{T} \int_{r}^{T} Z(t) \mathrm{d} t+\frac{1}{T} \int_{r}^{T} \int_{0}^{t} y(t-s) \mathrm{d} W(s) \mathrm{d} t
$$

for $T \in \mathbb{R}_{+}$with

$$
\begin{gathered}
I_{1}(T):=\frac{1}{T} \int_{r}^{T} y(t) \mathrm{d} t, \quad T \in \mathbb{R}_{+}, \\
Z(t):=\int_{[-r, 0]} \int_{u}^{0} y(t+u-s) X_{0}(s) \mathrm{d} s a(\mathrm{~d} u), \quad t \in[r, \infty) .
\end{gathered}
$$


By the Cauchy-Schwarz inequality and by (3.3.7),

$$
\begin{gathered}
\left|I_{1}(T)\right| \leqslant \sqrt{\int_{r}^{T} \frac{1}{T^{2}} \mathrm{~d} t \int_{r}^{T} y(t)^{2} \mathrm{~d} t} \leqslant \sqrt{\frac{1}{T} \int_{0}^{\infty} y(t)^{2} \mathrm{~d} t} \rightarrow 0, \\
\left|\frac{1}{T} \int_{r}^{T} Z(t) \mathrm{d} t\right| \leqslant \sqrt{\frac{1}{T} \int_{r}^{T} Z(t)^{2} \mathrm{~d} t} \leqslant \sqrt{\frac{r\|a\|}{T} \int_{-r}^{0} X_{0}(s)^{2} \mathrm{~d} s \int_{0}^{\infty} y(v)^{2} \mathrm{~d} v} \stackrel{\text { a.s. }}{\longrightarrow} 0
\end{gathered}
$$

as $T \rightarrow \infty$, hence we obtain (3.3.9). Moreover,

$$
\frac{1}{T} \int_{0}^{T} Y(t)^{2} \mathrm{~d} t=\frac{1}{T} \int_{0}^{r} Y(t)^{2} \mathrm{~d} t+I_{2}(T)+2 I_{3}(T)+\frac{1}{T} \int_{r}^{T}\left(\int_{0}^{t} y(t-s) \mathrm{d} W(s)\right)^{2} \mathrm{~d} t
$$

for $T \in \mathbb{R}_{+}$, with

$$
\begin{gathered}
I_{2}(T):=\frac{1}{T} \int_{r}^{T}\left(y(t) X_{0}(0)+\vartheta Z(t)\right)^{2} \mathrm{~d} t \\
I_{3}(T):=\frac{1}{T} \int_{r}^{T}\left(y_{i}(t) X_{0}(0)+\vartheta Z_{i}(t)\right)\left(\int_{0}^{t} y_{i}(t-s) \mathrm{d} W(s)\right) \mathrm{d} t .
\end{gathered}
$$

Again by (3.3.7),

$$
\begin{aligned}
0 & \leqslant I_{2}(T) \leqslant \frac{1}{T} \int_{r}^{T} 2\left(y(t)^{2} X_{0}(0)^{2}+\vartheta^{2} Z(t)^{2}\right) \mathrm{d} t \\
& \leqslant \frac{2 X_{0}(0)^{2}}{T} \int_{0}^{\infty} y(t)^{2} \mathrm{~d} t+\frac{2}{T} \vartheta^{2} r\|a\| \int_{-r}^{0} X_{0}(s)^{2} \mathrm{~d} s \int_{0}^{\infty} y(v)^{2} \mathrm{~d} v \stackrel{\text { a.s. }}{\longrightarrow} 0
\end{aligned}
$$

as $T \rightarrow \infty$, and

$$
\begin{aligned}
\left|I_{3}(T)\right| & \leqslant \frac{2}{T} \sqrt{\int_{r}^{T}\left(y(t) X_{0}(0)+\vartheta Z(t)\right)^{2} \mathrm{~d} t \int_{r}^{T}\left(\int_{0}^{t} y(t-s) \mathrm{d} W(s)\right)^{2} \mathrm{~d} t} \\
& =2 \sqrt{\frac{I_{2}(T)}{T} \int_{r}^{T}\left(\int_{0}^{t} y(t-s) \mathrm{d} W(s)\right)^{2} \mathrm{~d} t} \stackrel{\mathbb{P}}{\longrightarrow} 0 \quad \text { as } T \rightarrow \infty,
\end{aligned}
$$

hence we obtain (3.3.10).

3.3.11 Lemma. Let $y_{\ell}(t)=t^{\alpha_{\ell}} \operatorname{Re}\left(c_{\ell} \mathrm{e}^{\lambda_{\ell} t}\right), \quad t \in \mathbb{R}_{+}, \quad \ell \in\{1,2\}$, with some $\alpha_{\ell} \in \mathbb{Z}_{+}$, $c_{\ell}, \lambda_{\ell} \in \mathbb{C}$ with $\operatorname{Re}\left(\lambda_{\ell}\right) \in \mathbb{R}_{++}, \quad \ell \in\{1,2\}$. Let $\vartheta \in \mathbb{R}$. Suppose that $\left(Y_{\ell}(t)\right)_{t \in \mathbb{R}_{+}}, \quad \ell \in\{1,2\}$, are continuous stochastic processes admitting representation $(3.3 .8)$ on $[r, \infty)$ with $y=y_{\ell}$, $\ell \in\{1,2\}$, respectively. Then

$$
t^{-\alpha_{1}} \mathrm{e}^{-t \operatorname{Re}\left(\lambda_{1}\right)} Y_{1}(t)-\operatorname{Re}\left(c_{1} U_{\lambda_{1}}^{(\vartheta)} \mathrm{e}^{\mathrm{i} t \operatorname{Im}\left(\lambda_{1}\right)}\right) \stackrel{\text { a.s. }}{\longrightarrow} 0, \quad \text { as } t \rightarrow \infty,
$$

and

$$
\begin{aligned}
& T^{-\alpha_{1}-\alpha_{2}} \mathrm{e}^{-T \operatorname{Re}\left(\lambda_{1}+\lambda_{2}\right)} \int_{0}^{T} Y_{1}(t) Y_{2}(t) \mathrm{d} t \\
& -\int_{0}^{\infty} \mathrm{e}^{-t \operatorname{Re}\left(\lambda_{1}+\lambda_{2}\right)} \operatorname{Re}\left(c_{1} U_{\lambda_{1}}^{(\vartheta)} \mathrm{e}^{\mathrm{i}(T-t) \operatorname{Im}\left(\lambda_{1}\right)}\right) \operatorname{Re}\left(c_{2} U_{\lambda_{2}}^{(\vartheta)} \mathrm{e}^{\mathrm{i}(T-t) \operatorname{Im}\left(\lambda_{2}\right)}\right) \mathrm{d} t \stackrel{\text { a.s. }}{\longrightarrow} 0,
\end{aligned}
$$


as $T \rightarrow \infty$. Particularly, if $c_{1}, c_{2} \in \mathbb{R}$ and $\lambda_{1}, \lambda_{2} \in \mathbb{R}_{++}$, then

$$
\begin{gathered}
t^{-\alpha_{1}} \mathrm{e}^{-\lambda_{1} t} Y_{1}(t) \stackrel{\text { a.s. }}{\longrightarrow} c_{1} U_{\lambda_{1}}^{(\vartheta)}, \quad \text { as } t \rightarrow \infty, \\
T^{-\alpha_{1}-\alpha_{2}} \mathrm{e}^{-T\left(\lambda_{1}+\lambda_{2}\right)} \int_{0}^{T} Y_{1}(t) Y_{2}(t) \mathrm{d} t \stackrel{\text { a.s. }}{\longrightarrow} \frac{c_{1} c_{2}}{\lambda_{1}+\lambda_{2}} U_{\lambda_{1}}^{(\vartheta)} U_{\lambda_{1}}^{(\vartheta)}, \quad \text { as } T \rightarrow \infty .
\end{gathered}
$$

Proof. Note that

$$
t^{-\alpha_{1}} \mathrm{e}^{-t \operatorname{Re}\left(\lambda_{1}\right)} Y_{1}(t)-\operatorname{Re}\left(c_{1} U_{\lambda_{1}}^{(\vartheta)} \mathrm{e}^{\mathrm{i} t \operatorname{Im}\left(\lambda_{1}\right)}\right)=-I_{1}(t)+I_{2}(t)-I_{3}(t), \quad t \in[r, \infty),
$$

with

$$
\begin{aligned}
& I_{1}(t):=\vartheta \int_{[-r, 0]} \int_{v}^{0}\left[1-\left(1-\frac{s-v}{t}\right)^{\alpha_{1}}\right] \operatorname{Re}\left(c_{1} \mathrm{e}^{\mathrm{i} t \operatorname{Im}\left(\lambda_{1}\right)-\lambda_{1}(s-v)}\right) X_{0}(s) \mathrm{d} s a(\mathrm{~d} v), \\
& I_{2}(t):=\int_{0}^{t}\left[\left(1-\frac{s}{t}\right)^{\alpha_{1}}-1\right] \operatorname{Re}\left(c_{1} \mathrm{e}^{\mathrm{i} t \operatorname{Im}\left(\lambda_{1}\right)-\lambda_{1} s}\right) \mathrm{d} W(s), \\
& I_{3}(t):=\int_{t}^{\infty} \operatorname{Re}\left(c_{1} \mathrm{e}^{\mathrm{i} t \operatorname{Im}\left(\lambda_{1}\right)-\lambda_{1} s}\right) \mathrm{d} W(s) .
\end{aligned}
$$

By the dominated convergence theorem, $I_{1}(t) \stackrel{\text { a.s. }}{\longrightarrow} 0$ as $t \rightarrow \infty$. Moreover,

$$
I_{2}(t)=\sum_{k=1}^{\alpha_{1}}(-1)^{k}\left(\begin{array}{c}
\alpha_{1} \\
k
\end{array}\right) t^{-k} \int_{0}^{t} s^{k} \operatorname{Re}\left(c_{1} \mathrm{e}^{\mathrm{i} t \operatorname{Im}\left(\lambda_{1}\right)-\lambda_{1} s}\right) \mathrm{d} W(s) \stackrel{\text { a.s. }}{\longrightarrow} 0 \quad \text { as } t \rightarrow \infty
$$

by the strong law of martingales, see, e.g., Liptser and Shiryaev [33, Chapter $2, \S 6$, Theorem 10]. Obviously, $I_{3}(t) \stackrel{\text { a.s. }}{\longrightarrow} 0$ as $t \rightarrow \infty$, hence we obtain (3.3.12).

In order to prove (3.3.13), put

$$
V_{\ell}(t):=\operatorname{Re}\left(c_{\ell} U_{\lambda_{\ell}}^{(\vartheta)} \mathrm{e}^{\mathrm{i} t \operatorname{Im}\left(\lambda_{\ell}\right)}\right), \quad t \in \mathbb{R}, \quad \ell \in\{1,2\} .
$$

For each $T \in \mathbb{R}_{+}$, we have

$$
\int_{0}^{T} \mathrm{e}^{-t \operatorname{Re}\left(\lambda_{1}+\lambda_{2}\right)} V_{1}(T-t) V_{2}(T-t) \mathrm{d} t=\mathrm{e}^{-T \operatorname{Re}\left(\lambda_{1}+\lambda_{2}\right)} \int_{0}^{T} \mathrm{e}^{t \operatorname{Re}\left(\lambda_{1}+\lambda_{2}\right)} V_{1}(t) V_{2}(t) \mathrm{d} t,
$$

hence

$$
\begin{aligned}
& T^{-\alpha_{1}-\alpha_{2}} \mathrm{e}^{-T \operatorname{Re}\left(\lambda_{1}+\lambda_{2}\right)} \int_{0}^{T} Y_{1}(t) Y_{2}(t) \mathrm{d} t-\int_{0}^{\infty} \mathrm{e}^{-t \operatorname{Re}\left(\lambda_{1}+\lambda_{2}\right)} V_{1}(T-t) V_{2}(T-t) \mathrm{d} t \\
& =J_{1}(T)+J_{2}(T)+J_{3}(t)-J_{4}(T)-J_{5}(T)
\end{aligned}
$$


with

$$
\begin{aligned}
& J_{1}(T):=T^{-\alpha_{1}-\alpha_{2}} \mathrm{e}^{-T \operatorname{Re}\left(\lambda_{1}+\lambda_{2}\right)} \int_{0}^{T}\left[Y_{1}(t)-t^{\alpha_{1}} \mathrm{e}^{t \operatorname{Re}\left(\lambda_{1}\right)} V_{1}(t)\right]\left[Y_{2}(t)-t^{\alpha_{2}} \mathrm{e}^{t \operatorname{Re}\left(\lambda_{2}\right)} V_{2}(t)\right] \mathrm{d} t \\
& J_{2}(T):=T^{-\alpha_{1}-\alpha_{2}} \mathrm{e}^{-T \operatorname{Re}\left(\lambda_{1}+\lambda_{2}\right)} \int_{0}^{T}\left[Y_{1}(t)-t^{\alpha_{1}} \mathrm{e}^{t \operatorname{Re}\left(\lambda_{1}\right)} V_{1}(t)\right] t^{\alpha_{2}} \mathrm{e}^{t \operatorname{Re}\left(\lambda_{2}\right)} V_{2}(t) \mathrm{d} t \\
& J_{3}(T):=T^{-\alpha_{1}-\alpha_{2}} \mathrm{e}^{-T \operatorname{Re}\left(\lambda_{1}+\lambda_{2}\right)} \int_{0}^{T}\left[Y_{2}(t)-t^{\alpha_{2}} \mathrm{e}^{t \operatorname{Re}\left(\lambda_{2}\right)} V_{2}(t)\right] t^{\alpha_{1}} \mathrm{e}^{t \operatorname{Re}\left(\lambda_{1}\right)} V_{1}(t) \mathrm{d} t \\
& J_{4}(T):=\mathrm{e}^{-T \operatorname{Re}\left(\lambda_{1}+\lambda_{2}\right)} \int_{0}^{T}\left(1-\frac{t^{\alpha_{1}+\alpha_{2}}}{T^{\alpha_{1}+\alpha_{2}}}\right) \mathrm{e}^{t \operatorname{Re}\left(\lambda_{1}+\lambda_{2}\right)} V_{1}(t) V_{2}(t) \mathrm{d} t \\
& J_{5}(T):=\int_{T}^{\infty} \mathrm{e}^{-t \operatorname{Re}\left(\lambda_{1}+\lambda_{2}\right)} V_{1}(T-t) V_{2}(T-t) \mathrm{d} t
\end{aligned}
$$

By (3.3.12) and L'Hôspital's rule, $J_{1}(T) \stackrel{\text { a.s. }}{\longrightarrow} 0$ as $T \rightarrow \infty$. By the Cauchy-Schwarz inequality, $\left|J_{2}(T)\right| \leqslant \sqrt{J_{6}(T) J_{7}(T)}, \quad T \in \mathbb{R}_{+}$, where, by (3.3.12) and L'Hôspital's rule,

$$
J_{6}(T):=T^{-2 \alpha_{1}} \mathrm{e}^{-2 T \operatorname{Re}\left(\lambda_{1}\right)} \int_{0}^{T}\left[Y_{1}(t)-t^{\alpha_{1}} \mathrm{e}^{t \operatorname{Re}\left(\lambda_{1}\right)} V_{1}(t)\right]^{2} \mathrm{~d} t \stackrel{\text { a.s. }}{\longrightarrow} 0, \quad \text { as } T \rightarrow \infty,
$$

and

$$
\begin{aligned}
J_{7}(T) & :=T^{-2 \alpha_{2}} \mathrm{e}^{-2 T \operatorname{Re}\left(\lambda_{2}\right)} \int_{0}^{T} t^{2 \alpha_{2}} \mathrm{e}^{2 t \operatorname{Re}\left(\lambda_{2}\right)} V_{2}(t)^{2} \mathrm{~d} t \\
& =\int_{0}^{T}\left(1-\frac{t}{T}\right)^{2 \alpha_{2}} \mathrm{e}^{-2 t \operatorname{Re}\left(\lambda_{2}\right)} V_{2}(T-t)^{2} \mathrm{~d} t \leqslant \frac{1}{2 \operatorname{Re}\left(\lambda_{2}\right)} \sup _{t \in \mathbb{R}} V_{2}(t)^{2}<\infty \quad \text { a.s., }
\end{aligned}
$$

since $\left(V_{2}(t)\right)_{t \in \mathbb{R}}$ is a continuous and periodic process. Consequently, $J_{2}(T) \stackrel{\text { a.s. }}{\longrightarrow} 0$ as $T \rightarrow \infty$. In a similar way, $J_{3}(T) \stackrel{\text { a.s. }}{\longrightarrow} 0$ as $T \rightarrow \infty$, and

$$
\begin{aligned}
J_{4}(T) & \leqslant\left(\sup _{t \in \mathbb{R}}\left|V_{1}(t) V_{2}(t)\right|\right) \mathrm{e}^{-T \operatorname{Re}\left(\lambda_{1}+\lambda_{2}\right)} \int_{0}^{T}\left(1-\frac{t^{\alpha_{1}+\alpha_{2}}}{T^{\alpha_{1}+\alpha_{2}}}\right) \mathrm{e}^{t \operatorname{Re}\left(\lambda_{1}+\lambda_{2}\right)} \mathrm{d} t \\
& =\left(\sup _{t \in \mathbb{R}}\left|V_{1}(t) V_{2}(t)\right|\right) \int_{0}^{T}\left[1-\left(1-\frac{t}{T}\right)^{\alpha_{1}+\alpha_{2}}\right] \mathrm{e}^{-t \operatorname{Re}\left(\lambda_{1}+\lambda_{2}\right)} \mathrm{d} t \stackrel{\text { a.s. }}{\longrightarrow} 0
\end{aligned}
$$

as $T \rightarrow \infty$ by the dominated convergence theorem. Finally,

$$
\left|J_{5}(T)\right| \leqslant \frac{\mathrm{e}^{-T \operatorname{Re}\left(\lambda_{1}+\lambda_{2}\right)}}{\operatorname{Re}\left(\lambda_{1}+\lambda_{2}\right)} \sup _{t \in \mathbb{R}}\left|V_{1}(t) V_{2}(t)\right| \stackrel{\text { a.s. }}{\longrightarrow} 0 \quad \text { as } T \rightarrow \infty,
$$

hence we obtain (3.3.13).

Proof of Theorem 3.3.3. The continuous process $\left(Y^{(\vartheta)}(t)\right)_{t \in \mathbb{R}_{+}}$admits the representation (3.3.4) on $[r, \infty)$. The aim of the following discussion is to show that the function $\left(y_{\vartheta}(t)\right)_{t \in \mathbb{R}_{+}}$ is square integrable. Let $c \in\left(v_{\vartheta}^{*}, 0\right)$, and apply the representation (3.3.5). By the definition of 
$v_{\vartheta}^{*}$, we obtain $P_{\vartheta, \lambda}=0$ for each $\lambda \in \Lambda_{\vartheta}$ with $\operatorname{Re}(\lambda)>v_{\vartheta}^{*}$, and hence for each $\lambda \in \Lambda_{\vartheta}$ with $\operatorname{Re}(\lambda) \geqslant c$. Thus the representation (3.3.5) gives $y_{\vartheta}(t)=\mathrm{o}\left(\mathrm{e}^{c t}\right)$ as $t \rightarrow \infty$. Since $\left(y_{\vartheta}(t)\right)_{t \in \mathbb{R}_{+}}$ is continuous, the function $\left(\mathrm{e}^{-c t} y_{\vartheta}(t)\right)_{t \in \mathbb{R}_{+}}$is bounded, implying $\int_{0}^{\infty} y_{\vartheta}(t)^{2} \mathrm{~d} t<\infty$. Hence one can apply Lemma 3.3.10 to obtain

$$
J_{\vartheta, T}=\frac{1}{T} \int_{0}^{T} Y^{(\vartheta)}(t)^{2} \mathrm{~d} t=\frac{1}{T} \int_{0}^{r} Y^{(\vartheta)}(t)^{2} \mathrm{~d} t+\frac{1}{T} \int_{r}^{T} Y^{(\vartheta)}(t)^{2} \mathrm{~d} t \stackrel{\mathbb{P}}{\longrightarrow} \int_{0}^{\infty} y_{\vartheta}(t)^{2} \mathrm{~d} t=J_{\vartheta}
$$

as $T \rightarrow \infty$, where $J_{\vartheta}>0$, since $x_{0, \vartheta} \neq 0$ and $a \neq 0$ implies $y_{\vartheta} \neq 0$.

In case of $\vartheta=0$, we have $h_{0}(\lambda)=\lambda, \quad \Lambda_{0}=\{0\}, \quad m_{0}(0)=1$ and $P_{0,0}(t)=$ $A_{0,-1}(0) \int_{[-r, 0]} a(\mathrm{~d} u)=a([-r, 0]), \quad t \in \mathbb{R}$, since $1 / h_{0}(z)=z^{-1}$ yields $A_{0,-1}(0)=1$. The assumption $a([-r, 0])=0$ implies $P_{0,0}=0$, and hence $v_{0}^{*}=-\infty$ and $m_{0}^{*}=-\infty$. Moreover, the assumption $a([-r, 0])=0$ yields

$$
y_{0}(t)=\int_{[-r, 0]} x_{0,0}(t+u) a(\mathrm{~d} u)= \begin{cases}0 & \text { if } t \in[r, \infty), \\ a([-t, 0]) & \text { if } t \in[0, r],\end{cases}
$$

and one can obtain the formula for $J_{0}$.

Further, the process

$$
M^{(\vartheta)}(T):=\int_{0}^{T} Y^{(\vartheta)}(t) \mathrm{d} W(t), \quad T \in \mathbb{R}_{+},
$$

is a continuous martingale with $M^{(\vartheta)}(0)=0$ and with quadratic variation

$$
\left\langle M^{(\vartheta)}\right\rangle(T)=\int_{0}^{T} Y^{(\vartheta)}(t)^{2} \mathrm{~d} t
$$

hence Theorem VIII.5.42 of Jacod and Shiryaev [25] yields the statement.

Proof of Theorem 3.3.4. For each $T \in \mathbb{R}_{++}$, we have

$$
\Delta_{\vartheta, T}=\frac{1}{T^{m_{\vartheta}^{*}+1}} \int_{0}^{T} Y^{(\vartheta)}(t) \mathrm{d} W(t), \quad J_{\vartheta, T}=\frac{1}{T^{2\left(m_{\vartheta}^{*}+1\right)}} \int_{0}^{T} Y^{(\vartheta)}(t)^{2} \mathrm{~d} t .
$$

The continuous process $\left(Y^{(\vartheta)}(t)\right)_{t \in \mathbb{R}_{+}}$admits the representation $(3.3 .4)$ on $[r, \infty)$. We choose $c<0$ with $c>\sup \left\{\operatorname{Re}(\lambda): \lambda \in \Lambda_{\vartheta}, \operatorname{Re}(\lambda)<0\right\}$, and apply the representation (3.3.5). The assumption $v_{\vartheta}^{*}=0$ yields that $P_{\vartheta, \lambda}=0$ for each $\lambda \in \Lambda_{\vartheta}$ with $\operatorname{Re}(\lambda)>0$, hence we obtain

$$
y_{\vartheta}(t)=\sum_{\lambda \in \Lambda_{\vartheta} \cap(i \mathbb{R})} P_{\vartheta, \lambda}(t) \mathrm{e}^{\mathrm{i} t \operatorname{Im}(\lambda)}+\Psi_{\vartheta, c}(t), \quad t \in \mathbb{R}_{+}
$$

The leading term of the polynomial $P_{\vartheta, \lambda}$ is $c_{\vartheta, \lambda, m_{\vartheta}^{*}} t^{\widetilde{m}_{\vartheta}(\lambda)}$, thus, by the representation (3.3.4),

$$
Y^{(\vartheta)}(t)=\sum_{\substack{\lambda \in \Lambda_{\vartheta} \cap(\mathbb{R}) \\ \tilde{m}_{\vartheta}(\lambda)=m_{\vartheta}^{*}}} c_{\vartheta, \lambda, m_{\vartheta}^{*}} \mathrm{e}^{\mathrm{i} t \operatorname{Im}(\lambda)} \int_{0}^{t}(t-s)^{m_{\vartheta}^{*}} \mathrm{e}^{-\mathrm{i} s \operatorname{Im}(\lambda)} \mathrm{d} W(s)+\widetilde{Y}(t), \quad t \in \mathbb{R}_{+},
$$


where $(\widetilde{Y}(t))_{t \in \mathbb{R}_{+}}$is a continuous process satisfying

$$
\widetilde{Y}(t):=y_{\vartheta}(t) X_{0}(0)+\vartheta I_{\vartheta}(t)+I_{\Psi}(t)+S(t), \quad t \in[r, \infty),
$$

with

$$
\begin{gathered}
I_{\vartheta}(t):=\int_{[-r, 0]} \int_{v}^{0} y_{\vartheta}(t+v-s) X_{0}(s) \mathrm{d} s a(\mathrm{~d} v), \quad I_{\Psi}(t):=\int_{0}^{t} \Psi_{\vartheta, c}(t-s) \mathrm{d} W(s), \\
S(t):=\sum_{\lambda \in \Lambda_{\vartheta} \cap(\mathbb{i} \mathbb{R})} \sum_{\ell=0}^{\widetilde{m}_{\vartheta}(\lambda) \wedge\left(m_{\vartheta}^{*}-1\right)} c_{\vartheta, \lambda, \ell} I_{\ell}(t), \quad I_{\ell}(t):=\int_{0}^{t}(t-s)^{\ell} \mathrm{e}^{\lambda(t-s)} \mathrm{d} W(s),
\end{gathered}
$$

for $t \in[r, \infty)$ and $\ell \in \mathbb{Z}_{+}$. The aim of the following discussion is to show that $T^{-2\left(m_{\vartheta}^{*}+1\right)} \int_{r}^{T} \widetilde{Y}(t)^{2} \mathrm{~d} t \stackrel{\mathbb{P}}{\longrightarrow} 0$ as $T \rightarrow \infty$. First we show that $T^{-2\left(m_{\vartheta}^{*}+1\right)} \int_{r}^{T} y_{\vartheta}(t)^{2} \mathrm{~d} t \rightarrow 0$ as $T \rightarrow \infty$. For each $\lambda \in \Lambda_{\vartheta}$ with $\operatorname{Re}(\lambda)=0$, we have

$$
T^{-2\left(m_{\vartheta}^{*}+1\right)} \int_{r}^{T}\left|P_{\vartheta, \lambda}(t) \mathrm{e}^{\lambda t}\right|^{2} \mathrm{~d} t=T^{-2\left(m_{\vartheta}^{*}+1\right)} \int_{r}^{T}\left|P_{\vartheta, \lambda}(t)\right|^{2} \mathrm{~d} t \rightarrow 0 \quad \text { as } T \rightarrow \infty,
$$

since the degree of the complex-valued polynomial $P_{\vartheta, \lambda}$ is $\widetilde{m}_{\vartheta}(\lambda)$, which is at most $m_{\vartheta}^{*}$. Moreover,

$$
T^{-2\left(m_{\vartheta}^{*}+1\right)} \int_{r}^{T} \Psi_{\vartheta, c}(t)^{2} \mathrm{~d} t \leqslant T^{-2} \int_{r}^{T} \Psi_{\vartheta, c}(t)^{2} \mathrm{~d} t \leqslant T^{-2} \int_{r}^{\infty} \Psi_{\vartheta, c}(t)^{2} \mathrm{~d} t \rightarrow 0
$$

as $T \rightarrow \infty$, since $\int_{r}^{\infty}\left|\Psi_{\vartheta, c}(t)\right|^{2} \mathrm{~d} t<\infty$, because the function $\left(\mathrm{e}^{-c t}\left|\Psi_{\vartheta, c}(t)\right|\right)_{t \in \mathbb{R}_{+}}$is bounded. Thus, by the representation (3.3.14), one can obtain $T^{-2\left(m_{\vartheta}^{*}+1\right)} \int_{r}^{T} y_{\vartheta}(t)^{2} \mathrm{~d} t \rightarrow 0$ as $T \rightarrow \infty$. Next, by Lemma 3.3.9,

$$
\int_{r}^{T} I_{\vartheta}(t)^{2} \mathrm{~d} t \leqslant r\|a\| \int_{-r}^{0} X_{0}(s)^{2} \mathrm{~d} s \int_{0}^{T} y_{\vartheta}(v)^{2} \mathrm{~d} v
$$

hence we obtain $T^{-2\left(m_{\vartheta}^{*}+1\right)} \int_{r}^{T} I_{\vartheta}(t)^{2} \mathrm{~d} t \stackrel{\mathbb{P}}{\longrightarrow} 0$ as $T \rightarrow \infty$. Further, we have

$$
\begin{aligned}
& \mathbb{E}\left(\int_{r}^{T} I_{\Psi}(t)^{2} \mathrm{~d} t\right)=\int_{r}^{T}\left(\int_{0}^{t} \Psi_{\vartheta, c}(t-s)^{2} \mathrm{~d} s\right) \mathrm{d} t=\int_{r}^{T}\left(\int_{0}^{t} \Psi_{\vartheta, c}(u)^{2} \mathrm{~d} u\right) \mathrm{d} t \\
& =\int_{r}^{T} \Psi_{\vartheta, c}(u)^{2}\left(\int_{u}^{T} \mathrm{~d} t\right) \mathrm{d} u=\int_{r}^{T}(T-u) \Psi_{\vartheta, c}(u)^{2} \mathrm{~d} u \leqslant T \int_{r}^{T} \Psi_{\vartheta, c}(u)^{2} \mathrm{~d} u,
\end{aligned}
$$

hence $T^{-2} \int_{r}^{T} I_{\Psi}(t)^{2} \mathrm{~d} t \stackrel{\mathbb{P}}{\longrightarrow} 0$ as $T \rightarrow \infty$. Finally, for each $\lambda \in \Lambda_{\vartheta}$ with $\operatorname{Re}(\lambda)=0$ and each $\ell \in \mathbb{Z}_{+}$with $\ell \leqslant m_{\vartheta}^{*}-1$, we have $T^{-2\left(m_{\vartheta}^{*}+1\right)} \int_{0}^{T} I_{\ell}(t)^{2} \mathrm{~d} t \stackrel{\mathbb{P}}{\longrightarrow} 0$ as $T \rightarrow \infty$. Indeed,

$$
\begin{aligned}
& \frac{1}{T^{2\left(m_{\vartheta}^{*}+1\right)}} \mathbb{E}\left(\int_{0}^{T} I_{\ell}(t)^{2} \mathrm{~d} t\right)=\frac{1}{T^{2\left(m_{\vartheta}^{*}+1\right)}} \int_{0}^{T}\left(\int_{0}^{t}(t-s)^{2 \ell} \mathrm{d} s\right) \mathrm{d} t \\
& =\frac{1}{T^{2\left(m_{\vartheta}^{*}+1\right)}} \int_{0}^{T}\left(\int_{0}^{t} u^{2 \ell} \mathrm{d} u\right) \mathrm{d} t=\frac{1}{(2 \ell+1)(2 \ell+2) T^{2\left(m_{\vartheta}^{*}-\ell\right)}} \rightarrow 0 \quad \text { as } T \rightarrow \infty .
\end{aligned}
$$


Hence we conclude $T^{-2\left(m_{\vartheta}^{*}+1\right)} \int_{0}^{T} \widetilde{Y}(t)^{2} \mathrm{~d} t \stackrel{\mathbb{P}}{\longrightarrow} 0$ as $T \rightarrow \infty$.

Introduce the complex-valued processes $\left(Z_{\varphi, \ell}(t)\right)_{t \in \mathbb{R}_{+}}, \varphi \in \mathbb{R}, \quad \ell \in \mathbb{Z}_{+}$, by

$$
Z_{\varphi, \ell}(t):=\int_{0}^{t}(t-s)^{\ell} \mathrm{e}^{-\mathrm{i} \varphi s} \mathrm{~d} W(s) .
$$

Note that $Z_{0,0}=W$. For each $T \in \mathbb{R}_{++}$, consider the complex-valued processes $\left(Z_{\varphi, \ell}^{T}(t)\right)_{s \in[0,1]}$, $\varphi \in \mathbb{R}, \quad \ell \in \mathbb{Z}_{+}$, and the real-valued process $\left(X^{T}(s)\right)_{s \in[0,1]}$, defined by

$$
\begin{gathered}
Z_{\varphi, \ell}^{T}(s):=\frac{1}{T^{\ell+\frac{1}{2}}} Z_{\varphi, \ell}(T s)=\int_{0}^{s}(s-u)^{\ell} \mathrm{e}^{-\mathrm{i} T \varphi u} \mathrm{~d} W^{T}(u), \\
X^{T}(s):=\sum_{\substack{\lambda \in \Lambda_{\vartheta} \cap(\mathbb{R}) \\
\widetilde{m}_{\vartheta}(\lambda)=m_{\vartheta}^{*}}} c_{\vartheta, \lambda, m_{\vartheta}^{*}} \mathrm{e}^{\mathrm{i} T s \operatorname{Im}(\lambda)} Z_{\operatorname{Im}(\lambda), m_{\vartheta}^{*}}^{T}(s) .
\end{gathered}
$$

Then, for each $T \in \mathbb{R}_{++}$, we have

$$
Y^{(\vartheta)}(t)=T^{m_{\vartheta}^{*}+\frac{1}{2}} X^{T}\left(\frac{t}{T}\right)+\widetilde{Y}(t), \quad t \in \mathbb{R}_{+},
$$

and hence,

$$
\begin{gathered}
\Delta_{\vartheta, T}=\frac{1}{\sqrt{T}} \int_{0}^{T} X^{T}\left(\frac{t}{T}\right) \mathrm{d} W(t)+I_{1}(T)=\int_{0}^{1} X^{T}(s) \mathrm{d} W^{T}(s)+I_{1}(T), \\
J_{\vartheta, T}=\frac{1}{T} \int_{0}^{T}\left|X^{T}\left(\frac{t}{T}\right)\right|^{2} \mathrm{~d} t+2 I_{2}(T)+I_{3}(T)=\int_{0}^{1}\left|X^{T}(s)\right|^{2} \mathrm{~d} s+2 I_{2}(T)+I_{3}(T),
\end{gathered}
$$

with

$$
\begin{gathered}
I_{1}(T):=\frac{1}{T^{m_{\vartheta}^{*}+1}} \int_{0}^{T} \widetilde{Y}(t) \mathrm{d} W(t), \quad I_{2}(T):=\frac{1}{T^{m_{\vartheta}^{*}+\frac{3}{2}}} \int_{0}^{T} \operatorname{Re}\left(X^{T}\left(\frac{t}{T}\right) \overline{\widetilde{Y}(t)}\right) \mathrm{d} t, \\
I_{3}(T):=\frac{1}{T^{2\left(m_{\vartheta}^{*}+1\right)}} \int_{0}^{T}|\widetilde{Y}(t)|^{2} \mathrm{~d} t .
\end{gathered}
$$

Introducing the process

$$
Y^{T}(t):=\int_{0}^{t} X^{T}(s) \mathrm{d} W^{T}(s), \quad t \in \mathbb{R}_{+}, \quad T \in \mathbb{R}_{++},
$$

we have

$$
\int_{0}^{t} X^{T}(s)^{2} \mathrm{~d} s=\left[Y^{T}, Y^{T}\right](t), \quad t \in \mathbb{R}_{+}, \quad T \in \mathbb{R}_{++},
$$

where $([U, V](t))_{t \in \mathbb{R}_{+}}$denotes the quadratic covariation process of the processes $(U(t))_{t \in \mathbb{R}_{+}}$ and $(V(t))_{t \in \mathbb{R}_{+}}$. Moreover,

$$
\begin{aligned}
Y^{T}(t) & =\sum_{\substack{\lambda \in \Lambda_{\vartheta} \cap(\mathrm{i} \mathbb{R}) \\
\widetilde{m}_{\vartheta}(\lambda)=m_{\vartheta}^{*}}} c_{\vartheta, \lambda, m_{\vartheta}^{*}} \int_{0}^{t} \mathrm{e}^{\mathrm{i} T s \operatorname{Im}(\lambda)} Z_{\operatorname{Im}(\lambda), m_{\vartheta}^{*}}^{T}(s) \mathrm{d} W^{T}(s) \\
& =\sum_{\substack{\lambda \in \Lambda_{\vartheta} \cap(\mathrm{i} \mathbb{R}) \\
\widetilde{m}_{\vartheta}(\lambda)=m_{\vartheta}^{*}}} c_{\vartheta, \lambda, m_{\vartheta}^{*}} \int_{0}^{t} Z_{\operatorname{Im}(\lambda), m_{\vartheta}^{*}}^{T}(s) \mathrm{d} \overline{Z_{\operatorname{Im}(\lambda), 0}^{T}(s)} .
\end{aligned}
$$


By the functional central limit theorem,

$$
\left(Z_{\operatorname{Im}(\lambda), 0}^{T}: \lambda \in \Lambda_{\vartheta} \cap(\mathrm{i} \mathbb{R})\right) \stackrel{\mathcal{D}}{\longrightarrow}\left(\mathcal{Z}_{\operatorname{Im}(\lambda), 0}: \lambda \in \Lambda_{\vartheta} \cap(\mathrm{i} \mathbb{R})\right), \quad \text { as } T \rightarrow \infty
$$

since

$$
Z_{\varphi, 0}^{T}(s)=\int_{0}^{s} \cos (T \varphi u) \mathrm{d} W(u)-\mathrm{i} \int_{0}^{s} \sin (T \varphi u) \mathrm{d} W(u), \quad Z_{-\varphi, 0}^{T}(s)=\overline{Z_{\varphi, 0}^{T}(s)},
$$

for all $s \in[0,1], \varphi \in \mathbb{R}$ and $T \in \mathbb{R}_{++}$, and

$$
\begin{aligned}
& \int_{0}^{s} \cos \left(T \varphi_{1} u\right) \cos \left(T \varphi_{2} u\right) \mathrm{d} u=\frac{1}{2} \int_{0}^{s}\left[\cos \left(T\left(\varphi_{1}-\varphi_{2}\right) u\right)+\cos \left(T\left(\varphi_{1}+\varphi_{2}\right) u\right)\right] \mathrm{d} u \\
& = \begin{cases}\frac{s}{2}+\frac{\sin \left(2 T \varphi_{1} s\right)}{4 T \varphi_{1}} \rightarrow \frac{s}{2}, & \text { if } \varphi_{1}=\varphi_{2}, \\
\frac{\sin \left(T\left(\varphi_{1}-\varphi_{2}\right) s\right)}{2 T\left(\varphi_{1}-\varphi_{2}\right)}+\frac{\sin \left(T\left(\varphi_{1}+\varphi_{2}\right) s\right)}{2 T\left(\varphi_{1}+\varphi_{2}\right)} \rightarrow 0, & \text { if } \varphi_{1} \neq \varphi_{2},\end{cases} \\
& \int_{0}^{s} \sin \left(T \varphi_{1} u\right) \sin \left(T \varphi_{2} u\right) \mathrm{d} u=\frac{1}{2} \int_{0}^{s}\left[\cos \left(T\left(\varphi_{1}-\varphi_{2}\right) u\right)-\cos \left(T\left(\varphi_{1}+\varphi_{2}\right) u\right)\right] \mathrm{d} u \\
& = \begin{cases}\frac{s}{2}-\frac{\sin \left(2 T \varphi_{1} s\right)}{4 T \varphi_{1}} \rightarrow \frac{s}{2}, & \text { if } \varphi_{1}=\varphi_{2}, \\
\frac{\sin \left(T\left(\varphi_{1}-\varphi_{2}\right) s\right)}{2 T\left(\varphi_{1}-\varphi_{2}\right)}-\frac{\sin \left(T\left(\varphi_{1}+\varphi_{2}\right) s\right)}{2 T\left(\varphi_{1}+\varphi_{2}\right)} \rightarrow 0, & \text { if } \varphi_{1} \neq \varphi_{2},\end{cases} \\
& \int_{0}^{s} \sin \left(T \varphi_{1} u\right) \cos \left(T \varphi_{2} u\right) \mathrm{d} u=\frac{1}{2} \int_{0}^{s}\left[\sin \left(T\left(\varphi_{1}+\varphi_{2}\right) u\right)+\sin \left(T\left(\varphi_{1}-\varphi_{2}\right) u\right)\right] \mathrm{d} u \\
& = \begin{cases}\frac{1-\cos \left(2 T \varphi_{1} s\right)}{4 T \varphi_{1}} \rightarrow 0, & \text { if } \varphi_{1}=\varphi_{2}, \\
\frac{1-\cos \left(T\left(\varphi_{1}+\varphi_{2}\right) s\right)}{2 T\left(\varphi_{1}+\varphi_{2}\right)}+\frac{1-\cos \left(T\left(\varphi_{1}-\varphi_{2}\right) s\right)}{2 T\left(\varphi_{1}-\varphi_{2}\right)} \rightarrow 0, & \text { if } \varphi_{1} \neq \varphi_{2},\end{cases}
\end{aligned}
$$

as $T \rightarrow \infty$ for all $s \in[0,1]$ and $\varphi_{1}, \varphi_{2} \in \mathbb{R}_{+}$. Consequently,

$$
\left(Z_{\operatorname{Im}(\lambda), 0}^{T}, Z_{\operatorname{Im}(\lambda), m_{\vartheta}}^{T}: \lambda \in \Lambda_{\vartheta} \cap(\mathrm{i} \mathbb{R})\right) \stackrel{\mathcal{D}}{\longrightarrow}\left(\mathcal{Z}_{\operatorname{Im}(\lambda), 0}, \mathcal{Z}_{\operatorname{Im}(\lambda), m_{\vartheta}}: \lambda \in \Lambda_{\vartheta} \cap(\mathrm{i} \mathbb{R})\right), \quad \text { as } T \rightarrow \infty,
$$

and hence, by Itô's formula and the continuous mapping theorem,

$$
Y^{T} \stackrel{\mathcal{D}}{\longrightarrow} \mathcal{Y} \quad \text { as } T \rightarrow \infty
$$

with

$$
\mathcal{Y}(t)=\sum_{\substack{\lambda \in \Lambda_{\vartheta} \cap(\mathbb{R}) \\ \tilde{m}_{\vartheta}(\lambda)=m_{\vartheta}^{*}}} c_{\vartheta, \lambda, m_{\vartheta}^{*}} \int_{0}^{t} \mathcal{Z}_{\operatorname{Im}(\lambda), m_{\vartheta}^{*}}(s) \mathrm{d} \overline{\mathcal{Z}_{\operatorname{Im}(\lambda), 0}(s)}
$$

Further, by Corollary 4.12 in Gushchin and Küchler [16],

$$
\left(Y^{T}(1),\left[Y^{T}, Y^{T}\right](1)\right) \stackrel{\mathcal{D}}{\longrightarrow}(\mathcal{Y}(1),[\mathcal{Y}, \mathcal{Y}](1)) \quad \text { as } T \rightarrow \infty
$$


We also have

$$
\begin{aligned}
& \mathcal{Y}(t)=\mathbb{1}_{\left\{0 \in \Lambda_{\vartheta}, \widetilde{m}_{\vartheta}(0)=m_{\vartheta}^{*}\right\}} c_{\vartheta, 0, m_{\vartheta}^{*}} \int_{0}^{t} \mathcal{Z}_{0, m_{\vartheta}^{*}}(s) \mathrm{d} \mathcal{W}(s) \\
& +\frac{1}{\sqrt{2}} \sum_{\substack{\lambda \in \Lambda_{\vartheta} \cap\left(\mathbb{R}_{++}\right) \\
\tilde{m}_{\vartheta}(\lambda)=m_{\vartheta}^{*}}}\left[\int_{0}^{t}\left(c_{\vartheta, \lambda, m_{\vartheta}^{*}} \mathcal{Z}_{\operatorname{Im}(\lambda), m_{\vartheta}^{*}}(s)+\overline{c_{\vartheta, \lambda, m_{\vartheta}^{*}} \mathcal{Z}_{\operatorname{Im}(\lambda), m_{\vartheta}^{*}}(s)}\right) \mathrm{d} \mathcal{W}_{\operatorname{Im}(\lambda), \operatorname{Re}}(s)\right. \\
& \left.+\int_{0}^{t}\left(-c_{\vartheta, \lambda, m_{\vartheta}^{*}} \mathcal{Z}_{\operatorname{Im}(\lambda), m_{\vartheta}^{*}}(s)+\overline{c_{\vartheta, \lambda, m_{\vartheta}^{*}} \mathcal{Z}_{\operatorname{Im}(\lambda), m_{\vartheta}^{*}}(s)}\right) \mathrm{d} \mathcal{W}_{\operatorname{Im}(\lambda), \operatorname{Im}}(s)\right] \\
& =\mathbb{1}_{\left\{0 \in \Lambda_{\vartheta}, \widetilde{m}_{\vartheta}(0)=m_{\vartheta}^{*}\right\}} c_{\vartheta, 0, m_{\vartheta}^{*}} \int_{0}^{t} \mathcal{Z}_{0, m_{\vartheta}^{*}}(s) \mathrm{d} \mathcal{W}(s) \\
& +\sqrt{2} \sum_{\substack{\lambda \in \Lambda_{\vartheta} \cap\left(i \mathbb{R}_{++}\right) \\
\tilde{m}_{\vartheta}(\lambda)=m_{\vartheta}^{*}}}\left[\int_{0}^{t} \operatorname{Re}\left(c_{\vartheta, \lambda, m_{\vartheta}^{*}} \mathcal{Z}_{\operatorname{Im}(\lambda), m_{\vartheta}^{*}}(s)\right) \mathrm{d} \mathcal{W}_{\operatorname{Im}(\lambda), \operatorname{Re}}(s)\right. \\
& \left.-\int_{0}^{t} \operatorname{Im}\left(c_{\vartheta, \lambda, m_{\vartheta}^{*}} \mathcal{Z}_{\operatorname{Im}(\lambda), m_{\vartheta}^{*}}(s)\right) \mathrm{d} \mathcal{W}_{\operatorname{Im}(\lambda), \operatorname{Im}}(s)\right]
\end{aligned}
$$

hence

$$
\begin{aligned}
{[\mathcal{Y}, \mathcal{Y}](1)=} & \mathbb{1}_{\left\{0 \in \Lambda_{\vartheta}, \widetilde{m}_{\vartheta}(0)=m_{\vartheta}^{*}\right\}} c_{\vartheta, 0, m_{\vartheta}^{*}}^{2} \int_{0}^{1} \mathcal{Z}_{0, m_{\vartheta}^{*}}(s)^{2} \mathrm{~d} s \\
& +2 \sum_{\substack{\lambda \in \Lambda_{\vartheta} \cap\left(i \mathbb{R}_{++}\right) \\
\tilde{m}_{\vartheta}(\lambda)=m_{\vartheta}^{*}}}\left[\int_{0}^{1} \operatorname{Re}\left(c_{\vartheta, \lambda, m_{\vartheta}^{*}} \mathcal{Z}_{\operatorname{Im}(\lambda), m_{\vartheta}^{*}}(s)\right)^{2} \mathrm{~d} s+\int_{0}^{1} \operatorname{Im}\left(c_{\vartheta, \lambda, m_{\vartheta}^{*}} \mathcal{Z}_{\operatorname{Im}(\lambda), m_{\vartheta}^{*}}(s)\right)^{2} \mathrm{~d} s\right] \\
= & \sum_{\substack{\lambda \in \Lambda_{\vartheta} \cap(\mathbb{i}) \\
\widetilde{m}_{\vartheta}(\lambda)=m_{\vartheta}^{*}}}\left|c_{\vartheta, \lambda, m_{\vartheta}^{*}}\right|^{2} \int_{0}^{1}\left|\mathcal{Z}_{\operatorname{Im}(\lambda), m_{\vartheta}^{*}}(s)\right|^{2} \mathrm{~d} s .
\end{aligned}
$$

Recall that $I_{3}(T) \stackrel{\mathbb{P}}{\longrightarrow} 0$ as $T \rightarrow \infty$, which also implies $I_{1}(T) \stackrel{\mathbb{P}}{\longrightarrow} 0$ as $T \rightarrow \infty$. Further,

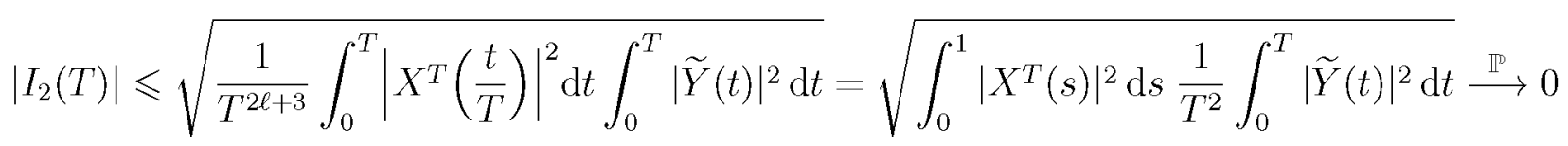

as $T \rightarrow \infty$, hence we obtain

$$
\left(\Delta_{\vartheta, T}, J_{\vartheta, T}\right) \stackrel{\mathcal{D}}{\longrightarrow}\left(\Delta_{\vartheta}, J_{\vartheta}\right) \quad \text { as } T \rightarrow \infty
$$

Moreover, we have $J_{\vartheta}>0$ almost surely. Indeed, $J_{\vartheta}=0$ would imply $\int_{0}^{1}\left|\mathcal{Z}_{\operatorname{Im}(\lambda), m_{\vartheta}^{*}}(s)\right|^{2} \mathrm{~d} s=0$ for all $\lambda \in\left\{\lambda \in \Lambda_{\vartheta} \cap(i \mathbb{R}): \widetilde{m}_{\vartheta}(\lambda)=m_{\vartheta}^{*}\right\}$, which, in turn, would imply $\mathcal{Z}_{\operatorname{Im}(\lambda), m_{\vartheta}^{*}}(s)=0$ for 
all $s \in[0,1]$. But this is in a contradiction with the fact that $\mathcal{Z}_{\operatorname{Im}(\lambda), m_{\vartheta}^{*}}$ is a non-degenerate Gaussian process.

Proof of Theorem 3.3.5. We have

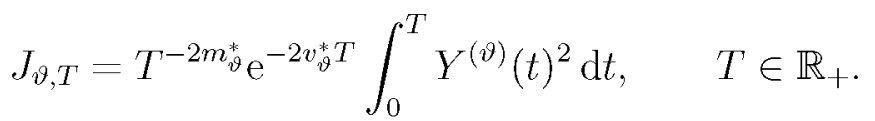

The process $\left(Y^{(\vartheta)}(t)\right)_{t \in[r, \infty)}$ admits the representation (3.3.4). We choose $c<v_{\vartheta}^{*}$ with $c>\sup \left\{\operatorname{Re}(\lambda): \lambda \in \Lambda_{\vartheta}, \operatorname{Re}(\lambda)<v_{\vartheta}^{*}\right\}$, and apply the representation (3.3.5). By the definition of $v_{\vartheta}^{*}$, one can obtain $P_{\vartheta, \lambda}=0$ for each $\lambda \in \Lambda_{\vartheta}$ with $\operatorname{Re}(\lambda)>v_{\vartheta}^{*}$, hence we obtain

$$
y_{\vartheta}(t)=\sum_{\lambda \in \Lambda_{\vartheta} \cap\left(v_{\vartheta}^{*}+\mathbf{i} \mathbb{R}\right)} \sum_{\ell=0}^{\widetilde{m}_{\vartheta}\left(v_{\vartheta}^{*}\right)} c_{\vartheta, \lambda, \ell} t^{\ell} \mathrm{e}^{\lambda t}+\Psi_{\vartheta, c}(t), \quad t \in \mathbb{R}_{+} .
$$

For each $\lambda \in \Lambda_{\vartheta}$ and $\ell \in\left\{0, \ldots, \widetilde{m}_{\vartheta}(\lambda)\right\}$, we have

$$
\begin{aligned}
c_{\vartheta, \lambda, \ell} t^{\ell} \mathrm{e}^{\lambda t}+c_{\vartheta, \bar{\lambda}, \ell} t^{\ell} \mathrm{e}^{\bar{\lambda} t} & =t^{\ell}\left(c_{\vartheta, \lambda, \ell} \mathrm{e}^{\lambda t}+\overline{c_{\vartheta, \lambda, \ell}} \mathrm{e}^{\lambda t}\right) \\
& =2 t^{\ell} \operatorname{Re}\left(c_{\vartheta, \lambda, \ell} \mathrm{e}^{\lambda t}\right)=t^{\ell}\left[\operatorname{Re}\left(c_{\vartheta, \lambda, \ell} \mathrm{e}^{\lambda t}\right)+\operatorname{Re}\left(c_{\vartheta, \bar{\lambda}, \ell} \mathrm{e}^{\bar{\lambda} t}\right)\right],
\end{aligned}
$$

hence (3.3.17) can also be written in the form

$$
y_{\vartheta}(t)=\sum_{\lambda \in \Lambda_{\vartheta} \cap\left(v_{\vartheta}^{*}+\mathrm{i} \mathbb{R}\right)} \sum_{\ell=0}^{\tilde{m}_{\vartheta}(\lambda)} t^{\ell} \operatorname{Re}\left(c_{\vartheta, \lambda, \ell} \mathrm{e}^{\lambda t}\right)+\Psi_{\vartheta, c}(t), \quad t \in \mathbb{R}_{+}
$$

Consequently, by the representation (3.3.4), we have

$$
Y^{(\vartheta)}(t)=Y(t)+\tilde{Y}(t), \quad t \in \mathbb{R}_{+},
$$

with

$$
Y(t):=\sum_{\lambda \in \Lambda_{\vartheta} \cap\left(v_{\vartheta}^{*}+\mathbb{R} \mathbb{R}\right)} \sum_{\ell=0}^{\widetilde{m}_{\vartheta}(\lambda)} Y_{\vartheta, \lambda, \ell}(t), \quad t \in \mathbb{R}_{+}
$$

where the continuous processes $\left(Y_{\vartheta, \lambda, \ell}(t)\right)_{t \in \mathbb{R}_{+}}, \lambda \in \Lambda, \quad \ell \in\left\{0, \ldots, \widetilde{m}_{\vartheta}\left(v_{\vartheta}^{*}\right)\right\}$, and $(\widetilde{Y}(t))_{t \in \mathbb{R}_{+}}$ admit representation $(3.3 .8)$ on $[r, \infty)$ with $y(t)=t^{\ell} \operatorname{Re}\left(c_{\vartheta, \lambda, \ell} \mathrm{e}^{\lambda t}\right), \lambda \in \Lambda, \ell \in\left\{0, \ldots, \widetilde{m}_{\vartheta}\left(v_{\vartheta}^{*}\right)\right\}$, and with $y(t)=\Psi_{\vartheta, c}(t), t \in \mathbb{R}_{+}$, respectively. The aim of the following discussion is to show that $T^{-2 m_{\vartheta}^{*}} \mathrm{e}^{-2 v_{\vartheta}^{*} T} \int_{0}^{T} \widetilde{Y}(t)^{2} \mathrm{~d} t \stackrel{\mathbb{P}}{\longrightarrow} 0$ as $T \rightarrow \infty$. We have

$$
\widetilde{Y}(t):=\Psi_{\vartheta, c}(t) X_{0}(0)+\vartheta I_{\vartheta}(t)+I_{\Psi}(t), \quad t \in[r, \infty),
$$

with

$$
I_{\vartheta}(t):=\int_{[-r, 0]} \int_{v}^{0} \Psi_{\vartheta, c}(t+v-s) X_{0}(s) \mathrm{d} s a(\mathrm{~d} v), \quad I_{\Psi}(t):=\int_{0}^{t} \Psi_{\vartheta, c}(t-s) \mathrm{d} W(s) .
$$


The function $\left(\mathrm{e}^{-c t} \Psi_{\vartheta, c}(t)\right)_{t \in \mathbb{R}_{+}}$is bounded, hence $\left|\Psi_{\vartheta, c}(t)\right| \leqslant C \mathrm{e}^{c t}$ for all $t \in \mathbb{R}_{+}$with $C:=\sup _{t \in \mathbb{R}_{+}} \mathrm{e}^{-c t}\left|\Psi_{\vartheta, c}(t)\right|<\infty$, hence

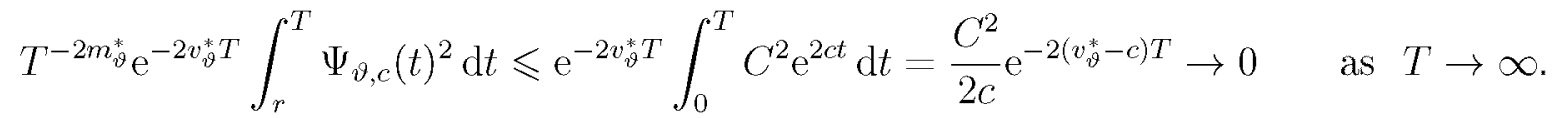

Next, by Lemma 3.3.9,

$$
\int_{r}^{T} I_{\vartheta}(t)^{2} \mathrm{~d} t \leqslant r\|a\| \int_{-r}^{0} X_{0}(s)^{2} \mathrm{~d} s \int_{0}^{T} \psi_{\vartheta}(v)^{2} \mathrm{~d} v
$$

hence one can obtain $T^{-2 m_{\vartheta}^{*}} \mathrm{e}^{-2 v_{\vartheta}^{*} T} \int_{0}^{T} I_{\vartheta}(t)^{2} \mathrm{~d} t \stackrel{\mathbb{P}}{\longrightarrow} 0$ as $T \rightarrow \infty$. Finally, by (3.3.16), $T^{-2 m_{\vartheta}^{*}} \mathrm{e}^{-2 v_{\vartheta}^{*} T} \int_{0}^{T} I_{\Psi}(t)^{2} \mathrm{~d} t \stackrel{\mathbb{P}}{\longrightarrow} 0$ as $T \rightarrow \infty$, and we conclude $T^{-2 m_{\vartheta}^{*}} \mathrm{e}^{-2 v_{\vartheta}^{*} T} \int_{0}^{T} \widetilde{Y}(t)^{2} \mathrm{~d} t \stackrel{\mathbb{P}}{\longrightarrow} 0$ as $T \rightarrow \infty$.

Applying Lemma 3.3.11, one can obtain

$$
\begin{aligned}
& T^{-\ell_{1}-\ell_{2}} \mathrm{e}^{-2 v_{\vartheta}^{*} T} \int_{0}^{T} Y_{\vartheta, \lambda_{1}, \ell_{1}}(t) Y_{\vartheta, \lambda_{2}, \ell_{2}}(t) \mathrm{d} t \\
& -\int_{0}^{\infty} \mathrm{e}^{-2 v_{\vartheta}^{*} t} \operatorname{Re}\left(c_{\vartheta, \lambda_{1}, \ell_{1}} U_{\lambda_{1}}^{(\vartheta)} \mathrm{e}^{\mathrm{i}(T-t) \operatorname{Im}\left(\lambda_{1}\right)}\right) \operatorname{Re}\left(c_{\vartheta, \lambda_{2}, \ell_{2}} U_{\lambda_{2}}^{(\vartheta)} \mathrm{e}^{\mathrm{i}(T-t) \operatorname{Im}\left(\lambda_{2}\right)}\right) \mathrm{d} t \stackrel{\text { a.s. }}{\longrightarrow} 0
\end{aligned}
$$

as $T \rightarrow \infty$ for each $\lambda_{1}, \lambda_{2} \in \Lambda$ with $\operatorname{Re}\left(\lambda_{1}\right)=\operatorname{Re}\left(\lambda_{2}\right)=v_{\vartheta}^{*}$ and $\ell_{1} \in\left\{0, \ldots, \widetilde{m}_{\vartheta}\left(\lambda_{1}\right)\right\}$, $\ell_{2} \in\left\{0, \ldots, \widetilde{m}_{\vartheta}\left(\lambda_{2}\right)\right\}$. Consequently,

$$
T^{-2 m_{\vartheta}^{*}} \mathrm{e}^{-2 v_{\vartheta}^{*} T} \int_{0}^{T} Y(t)^{2} \mathrm{~d} t-\int_{0}^{\infty} \mathrm{e}^{-2 v_{\vartheta}^{*} t}\left(\sum_{\lambda \in \Lambda_{\vartheta} \cap\left(v_{\vartheta}^{*}+\mathrm{i} \mathbb{R}\right)} \operatorname{Re}\left(c_{\vartheta, \lambda, m_{\vartheta}^{*}} U_{\lambda}^{(\vartheta)} \mathrm{e}^{\mathrm{i}(T-t) \operatorname{Im}(\lambda)}\right)\right)^{2} \mathrm{~d} t \stackrel{\text { a.s. }}{\longrightarrow} 0
$$

as $T \rightarrow \infty$, hence we obtain $J_{\vartheta, T}-J_{\vartheta}(T) \stackrel{\text { a.s. }}{\longrightarrow} 0$ as $T \rightarrow \infty$. Since $H_{\vartheta} \neq \emptyset$ and the numbers in $H_{\vartheta}$ have a common divisor $D_{\vartheta}$, the process $\left(J_{\vartheta}(t)\right)_{t \in \mathbb{R}_{+}}$is periodic with period $\frac{2 \pi}{D_{\vartheta}}$, and, by Theorem VIII.5.42 of Jacod and Shiryaev [25], one can conclude

$$
\left(\Delta_{\vartheta, k D+d}, J_{\vartheta, k D+d}\right) \stackrel{\mathcal{D}}{\longrightarrow}\left(\Delta_{\vartheta}(d), J_{\vartheta}(d)\right) \quad \text { as } k \rightarrow \infty
$$

for all $d \in\left[0, \frac{2 \pi}{D_{\vartheta}}\right)$. Moreover, we have $J_{\vartheta}(d)>0$ almost surely for all $d \in\left[0, \frac{2 \pi}{D_{\vartheta}}\right)$. Indeed, if $J_{\vartheta}(d)=0$ almost surely for all $d \in\left[0, \frac{2 \pi}{D_{\vartheta}}\right)$, then

$$
\operatorname{Re}\left(\sum_{\substack{\lambda \in \Lambda_{\vartheta} \cap\left(v_{\vartheta}^{*}+\mathrm{iR}\right) \\ \widetilde{m}_{\vartheta}(\lambda)=m_{\vartheta}^{*}}} c_{\vartheta, \lambda, m_{\vartheta}^{*}} U_{\lambda}^{(\vartheta)} \mathrm{e}^{\mathrm{i}(d-t) \operatorname{Im}(\lambda)}\right)=0
$$

for all $d \in\left[0, \frac{2 \pi}{D_{\vartheta}}\right)$ and $t \in \mathbb{R}_{++}$. But this is in a contradiction with the fact that the left-hand side is a Gaussian random variable with variance

$$
\int_{0}^{\infty} \operatorname{Re}\left(\sum_{\lambda \in \Lambda_{\vartheta} \cap\left(v_{\vartheta}^{*}+\mathrm{i} \mathbb{R}\right)} c_{\vartheta, \lambda, m_{\vartheta}^{*}} \mathrm{e}^{\mathrm{i}(d-t) \operatorname{Im}(\lambda)} \mathrm{e}^{-\lambda s}\right)^{2} \mathrm{~d} s \neq 0
$$


Consequently we obtain that the family $\left(\mathcal{E}_{T}\right)_{T \in \mathbb{R}_{++}}$is PLAMN at $\vartheta$.

If $H_{\vartheta}=\emptyset$, then $\Lambda_{\vartheta} \cap\left(v_{\vartheta}^{*}+i \mathbb{R}\right)=\left\{v_{\vartheta}^{*}\right\}$ and $\widetilde{m}_{\vartheta}\left(v_{\vartheta}^{*}\right)=m_{\vartheta}^{*}$, thus

$$
T^{-2 m_{\vartheta}^{*}} \mathrm{e}^{-2 v_{\vartheta}^{*} T} \int_{0}^{T} Y(t)^{2} \mathrm{~d} t \stackrel{\text { a.s. }}{\longrightarrow} \int_{0}^{\infty} \mathrm{e}^{-2 v_{\vartheta}^{*} t}\left(c_{\vartheta, v_{\vartheta}^{*}, m_{\vartheta}^{*}} U_{\lambda}^{(\vartheta)}\right)^{2} \mathrm{~d} t=J_{\vartheta}, \quad \text { as } T \rightarrow \infty .
$$

By Theorem VIII.5.42 of Jacod and Shiryaev [25], one can conclude

$$
\left(\Delta_{\vartheta, T}, J_{\vartheta, T}\right) \stackrel{\mathcal{D}}{\longrightarrow}\left(\Delta_{\vartheta}, J_{\vartheta}\right) \quad \text { as } T \rightarrow \infty,
$$

and we obtain that the family $\left(\mathcal{E}_{T}\right)_{T \in \mathbb{R}_{++}}$is LAMN at $\vartheta$. 



\section{Appendix A}

\section{Summary}

In this thesis a statistical model of linear stochastic differential equation with time delay is considered. The aim of the investigation is to prove local asymptotic properties of the likelihood function. The thesis contains three chapters. Chapter 1 is an introduction. Section 1.1 includes some applications for deterministic and stochastic differential equations with time delay. These are the delayed logistic or Hutchinson's equation and the Wright's equation. Further references are mentioned on applications for deterministic equation in biology, chemistry, physics, economics and epidemics. To illustrate an application for stochastic delay differential equation, a financial application is presented by Appleby, Riedle és Swords [2], which was one of the most motivational example for the birth of this thesis. In this, a model is constructed to describe the dynamics of a risky security, in which the traders of the security determine their investment strategy by historical information. The central element of the derived model is the equation (1.1.5)

$$
\mathrm{d} Y(t)=L\left(Y_{t}\right) \mathrm{d} t+\sigma \mathrm{d} W(t), \quad t \geq 0,
$$

where $L$ is a linear functional and $W$ is a standard Wiener process. The main result of this thesis is connected to similar equations. Section 1.2 contains an introduction for the local asymptotic properties of the likelihood function. The basic concept of asymptotic statistics is the local asymptotic normality. In this part we give a heuristically description on this with the help of the book by van der Vaart [46]. Finally, in Section 1.3 a historical background of the earlier results connected to investigation of the local asymptotic properties of statistical models described by stochastic delay differential equations can be found.

Chapter 2 contains on the one hand the basic notions and results of asymptotic statistics, which are needed to understand the main results of this thesis. These are the definitions of statistical experiment, convergence of statistical experiments, LAN, LAMN, LAQ and PLAMN, and the Le Cam's lemmas, which can be found in Section 2.1. In Section 2.2 some useful consequences of the local asymptotic properties are stated, namely the asymptotic behaviour of the maximum likelihood estimator and the questions of the efficiency of estimators and optimality of tests.

On the other hand, an important part of the thesis is Section 2.3 , where a well-known 
financial model, the Heston model is considered, and investigated from asymptotic statistics point of view. This is a new result, which has been published in Benke and Pap [12]. The model is the following

$$
\left\{\begin{array}{l}
\mathrm{d} Y_{t}=\left(a-b Y_{t}\right) \mathrm{d} t+\sigma_{1} \sqrt{Y_{t}} \mathrm{~d} W_{t} \\
\mathrm{~d} X_{t}=\left(\alpha-\beta Y_{t}\right) \mathrm{d} t+\sigma_{2} \sqrt{Y_{t}}\left(\varrho \mathrm{d} W_{t}+\sqrt{1-\varrho^{2}} \mathrm{~d} B_{t}\right), \quad t \geqslant 0
\end{array}\right.
$$

where $a>0, b, \alpha, \beta \in \mathbb{R}, \sigma_{1}>0, \sigma_{2}>0, \varrho \in(-1,1)$ and $\left(W_{t}, B_{t}\right)_{t \geqslant 0}$ is a 2-dimensional standard Wiener process. Here one can interpret $X_{t}$ as the log-price of an asset, and $Y_{t}$ as the stochastic volatility of the asset price at time $t \geqslant 0$. One can distinguish three cases: subcritical if $b>0$, critical if $b=0$ and supercritical if $b<0$. We study local asymptotic properties of the likelihood ratios of this model concerning the drift parameter $(a, \alpha, b, \beta)$. We prove LAN in the subcritical case and LAQ in the critical case. In a submodel of the critical case we prove a stronger result, namely LAN property holds. The most interesting case is the supercritical one, because in this case we prove, that LAQ does not hold, although the limit distribution of the likelihood function exists and we can describe it. As in the critical case, in a submodel of the supercritical case we have a stronger result, namely LAMN property holds.

In Chapter 3 the main results of this thesis are presented. We assume that we have a stochastic process $(X(t))_{t \in[-r, T]}$, which satisfies the linear stochastic delay differential equation

$$
\mathrm{d} X(t)=\vartheta \int_{[-r, 0]} X(t+u) a(\mathrm{~d} u) \mathrm{d} t+\mathrm{d} W(t), \quad t \geqslant 0,
$$

where $a$ is a finite signed measure on $[-r, 0], W$ is a standard Wiener process and $\vartheta$ is the unknown, real parameter. In Section 3.1 we make preliminaries to present the earlier results, which are needed. An important one is the so-called variation of constants formula (3.1.5), which is helpful to view the diffusion term in the stochastic differential equation as an inhomogeneous term in a deterministic differential equation. In addition, we can represent the solution of the stochastic differential equation in a term of the so-called fundamental solution of the corresponding deterministic differential equation. An other crucial fact is, that we can describe the asymptotic behaviour of the fundamental solution (Lemma 3.1.1).

Section 3.2 contains a special case, when the delay is uniform, namely when the measure $a$ is the Lebesgue measure and $r=1$. The results have been published in Benke and Pap [10]. In this special case we can determine the exact values of the parameter, where the appropriate local asymptotic property is valid. We prove LAN, when $\vartheta \in\left(-\pi^{2} / 2,0\right)$, LAMN holds, if $\vartheta \in(0, \infty)$ and we prove PLAMN, when $\vartheta \in\left(-\infty,-\pi^{2} / 2\right)$. At the two critical values of the parameter, $-\pi^{2} / 2$ and 0 we show the LAQ property.

In Section 3.3 the main results of the general case are presented. The results have been published in Benke and Pap [11]. In this general model it can not be possible to determine the exact values of the parameter $\vartheta$, where the appropriate local asymptotic property is valid. The aim is to give sufficient conditions for this. In the uniform delay case and in each of the earlier results, which concern local asymptotic properties of delayed models, LAN has been 
proved in case of $v_{0}(\vartheta)<0$, where $v_{0}(\vartheta)$ is the real part of the rightmost characteristic root of the corresponding deterministic differential equation. Accordingly one can imagine, that this condition would be the sufficient for LAN. But it turns out, that is not the truth. We give an example, where $v_{0}(\vartheta)=0$, and LAN holds. A modification is needed to give the proper condition (see, the definition of $v_{\vartheta}^{*}$, Definition 3.3.1). We prove LAN, if $v_{\vartheta}^{*}<0$ and LAQ, if $v_{\vartheta}^{*}=0$. Finally, if $v_{\vartheta}^{*}>0$, then under some additional conditions, LAMN or PLAMN is valid. 



\section{Appendix B}

\section{Összefoglaló}

Az értekezésben lineáris idókésleltetett sztochasztikus differenciálegyenletekkel leírt statisztikai modellt tekintünk. A vizsgálódás célja a likelihood függvény lokális aszimptotikus tulajdonságainak bizonyítása. Az értekezés három fejezetbôl áll. Az elsô fejezet (Chapter 1) egy bevezetô. Elsố szakaszában (Section 1.1) néhány példa, illetve alkalmazás szerepel időkésleltetést tartalmazó determinisztikus és sztochasztikus differenciálegyenletekre. Ezek között szerepel a késleltetett logisztikus-, avagy Hutchinson-egyenlet (1.1.1) és a Wright-egyenlet (1.1.2). Hivatkozások szerepelnek még további determinisztikus példákra a biológia, kémia, fizika, közgazdaságtan és epidemiológia területén. Késleltetett sztochasztikus differenciálegyenletre egy pénzügyi alkalmazás szerepel Appleby, Riedle és Swords [2] cikke nyomán, ami az egyik legfontosabb motivációja az értekezésnek. Ebben a szerzók egy olyan modellt írnak fel egy kockázatos eszköz árfolyamatára, melyben a dinamika részben abból ered, hogy a kereskedók múltbeli információk alapján alakítják kereskedési stratégiájukat. A kapott modell központi építőeleme az (1.1.5) egyenlet:

$$
\mathrm{d} Y(t)=L\left(Y_{t}\right) \mathrm{d} t+\sigma \mathrm{d} W(t), \quad t \geq 0,
$$

ahol $L$ egy lineáris funkcionál, $W$ pedig egy standard Wiener-folyamat. Az értekezés fö eredményében ehhez hasonló egyenletet tekintünk. Ezt követő́en a bevezetô második szakaszában (Section 1.2) a lokális aszimptotikus tulajdonságokra adunk egy bevezetőt. Ebben az aszimptotikus statisztika egy alapvető fogalmára a lokális aszimptotikus normalitásra adunk egy heurisztikus leírást van der Vaart [46] könyvének segítségével. Végül a harmadik szakaszban (Section 1.3) egy történeti áttekintést adunk azon korábbi eredményekröl, amelyek késleltetett sztochasztikus differenciálegyenletekkel leírt statisztikai modellek lokális aszimptotikus tulajdonságait vizsgálják.

A második fejezetben (Chapter 2) egyrészt az aszimptotikus statisztika azon alapvető fogalmai és eredményei szerepelnek, melyek az értekezés fő eredményének megértéséhez szükségesek. Ide tartoznak a statisztikai kísérletek és ezek konvergenciájának definíciója, a LAN, LAMN, LAQ és PLAMN tulajdonságok definíciói, illetve Le Cam lemmái. Ezeket tartalmazza az első szakasz (Section 2.1). A második szakaszban (Section 2.2) néhány fontos következmény található, ami a különböző lokális aszimptotikus tulajdonságok teljesülése esetén adódnak. Ezek a 
maximum likelihood becslés aszimptotikus tulajdonságai, illetve becslések hatásosságának, és tesztek aszimptotikus optimalitásának témakörei.

Másrészt a fejezet másik fontos része a harmadik szakasz (Section 2.3), amelyben egy híres pénzügyi modellt, a Heston-modellt tekintjük, és vizsgáljuk meg aszimptotikus statisztikai szempontból. Ez új eredmény, ami Benke és Pap [12] cikkében került publikálásra. A modell az alábbi formában írható fel.

$$
\left\{\begin{array}{l}
\mathrm{d} Y_{t}=\left(a-b Y_{t}\right) \mathrm{d} t+\sigma_{1} \sqrt{Y_{t}} \mathrm{~d} W_{t} \\
\mathrm{~d} X_{t}=\left(\alpha-\beta Y_{t}\right) \mathrm{d} t+\sigma_{2} \sqrt{Y_{t}}\left(\varrho \mathrm{d} W_{t}+\sqrt{1-\varrho^{2}} \mathrm{~d} B_{t}\right), \quad t \geqslant 0
\end{array}\right.
$$

ahol $a>0, b, \alpha, \beta \in \mathbb{R}, \sigma_{1}>0, \sigma_{2}>0, \varrho \in(-1,1)$ és $\left(W_{t}, B_{t}\right)_{t \geqslant 0}$ egy kétdimenziós standard Wiener-folyamat. Ebben a pénzügyi modellben $X_{t}$ írja le egy kockázatos eszköz $t$ időpontbeli árfolyamának logaritmusát, és $Y_{t}$ pedig a sztochasztikus volatilitását. A $b$ paraméter előjelétől függően három esetet lehet megkülönböztetni, melyeket szubkritikus $(b>0)$, kritikus $(b=0)$ és szuperkritikus $(b<0)$ esetnek nevezzük. A $\boldsymbol{\theta}=(a, \alpha, b, \beta)$ paramétert tekintve a következō eredményeket bizonyítjuk. A szubkritikus esetben LAN tulajdonságot látunk be. A kritikus esetben az egész statisztikai modellre a LAQ tulajdonság teljesül, viszont egy részmodellben a LAN tulajdonságot bizonyítjuk. A legérdekesebb eset talán a szuperkritikus eset, hiszen ebben az esetben bebizonyítjuk, hogy nem teljesül a LAQ tulajdonság, holott a likelihood függvény kérdéses elemeinek van határeloszlása. Úgy ahogy a kritikus esetben, itt is egy részmodellben többet lehet állítani, LAMN tulajdonságot látunk be.

A harmadik fejezetben (Chapter 3) az értekezés fô eredményei szerepelnek. A vizsgált lineáris késleltetett sztochasztikus differenciálegyenlet az alábbi alakú.

$$
\mathrm{d} X(t)=\vartheta \int_{[-r, 0]} X(t+u) a(\mathrm{~d} u) \mathrm{d} t+\mathrm{d} W(t), \quad t \geqslant 0,
$$

ahol a egy véges, elöjeles mérték a $[-r, 0]$ intervallumon, $W$ egy standard Wiener-folyamat, és $\vartheta$ az ismeretlen, valós paraméter. Az első szakaszban (Section 3.1) előkészületeket teszünk, melyben ismertetjük a szükséges korábbi eredményeket. Fontos eszköz az ún. konstansvariációs formula (3.1.5), melynek segítségével a sztochasztikus differenciálegyenletekben szereplö diffúziós tagot úgy képzelhetjük el mint a determinisztikus egyenletek esetében egy inhomogén tagot. Ezáltal a vizsgált sztochasztikus egyenlet megoldását felírhatjuk a kapcsolatos determinisztikus egyenlet ún. fundamentális megoldásának segítségével. Másik központi jelentőségû tény, hogy ezen fundamentális megoldás aszimptotikus viselkedését megfelelően le tudjuk írni (Lemma 3.1.1).

A második szakaszban (Section 3.2) egy speciális esetet vizsgálunk, amikor a késleltetés egyenletes, azaz amikor az a mérték a Lebesgue-mérték, és $r=1$. Ezen modellhez tartozó eredmények Benke és Pap [10] cikkében kerültek publikálásra. Ennek a speciális esetnek az az előnye, hogy pontosan le lehet írni a $\vartheta$ paraméter azon értékeit, amelyek esetén a különböző lokális aszimptotikus tulajdonságok teljesülnek. A LAN tulajdonságot bizonyítjuk, amikor $\vartheta \in\left(-\pi^{2} / 2,0\right)$, LAMN tulajdonság teljesül, ha $\vartheta \in(0, \infty)$, valamint PLAMN, ha 
$\vartheta \in\left(-\infty,-\pi^{2} / 2\right)$. A két kritikus paraméterérték, $-\pi^{2} / 2$ és 0 esetén LAQ tulajdonságot bizonyítunk.

A harmadik szakaszban (Section 3.3) tárgyaljuk az általános esetet. Az eredmények Benke és Pap [11] cikkében kerültek publikálásra. Itt már természetesen nincs esély arra, hogy pontosan meghatározzuk a paraméter azon értékeit, amelyek esetén a különbözô lokális aszimptotikus tulajdonságok teljesülnek. Viszont adunk rá elégséges feltételeket. A korábbi eredményekben, és az egyenletes késleltetés esetében is azt látható, hogy a LAN tulajdonság akkor teljesül, ha a $v_{0}(\vartheta)$ mennyiség - ami a kapcsolatos determinisztikus egyenlet maximális valósrészû karakterisztikus gyökének valós része - szigorúan negativ. Ennek megfelelổen az sejthető, hogy ez a feltétele a LAN tulajdonság teljesülésének. Ezzel szemben mutatunk példát olyan esetre, amikor $v_{0}(\vartheta)=0$, és a LAN tulajdonság áll fenn. A $v_{0}(\vartheta)$ mennyiség egy módosításával (lásd a $\quad v_{\vartheta}^{*}$ mennyiség definícióját, Definition 3.3.1) tudunk megfelelö feltételeket felírni a lokális aszimptotikus tulajdonságok teljesüléséhez. Amennyiben $v_{\vartheta}^{*}<0$, a LAN tulajdonság teljesül, ha $v_{\vartheta}^{*}=0$, akkor LAQ tulajdonságot lehet bizonyítani. Végül $v_{\vartheta}^{*}>0$ esetén egy további feltételtől függően LAMN, illetve PLAMN tulajdonság áll fenn. 



\section{Bibliography}

[1] Appleby, J. A. D., Mao, X. and Riedle, M. (2009). Geometric Brownian motion with delay: mean square characterisation. Proceedings of the American Mathematical Society 137(1) 339-348.

[2] Appleby, J. A. D., Riedle, M. and Swords, C. (2013). Bubbles and crashes in a Black-Scholes model with delay. Finance and Stochastic 17(1) 1-30.

[3] Arriojas, M., Hu, Y., Mohammed, S.-E. and Pap, G. (2007). A delayed Black and Scholes formula Stochastic Analysis and Applications 25(2) 471-492.

[4] Bánhelyi, B., Csendes, T., Krisztin, T. and Neumaier, A. (2014). Global Attractivity of the Zero Solution for Wright's Equation. Siam Journal on Applied Dynamical Systems 13(1) 537-563.

[5] Barczy, M., Döring, L., Li, Z. and PAP, G. (2013). On parameter estimation for critical affine processes. Electronic Journal of Statistics 7 647-696.

[6] BARCZy, M., Döring, L., Li, Z. and PAP, G. (2014). Stationarity and ergodicity for an affine two factor model. Advances in Applied Probability. 46(3) 878-898.

[7] Barczy, M. and PAp, G. (2016). Asymptotic properties of maximum likelihood estimators for Heston models based on continuous time observations. Statistics. 50(2) 389-417.

[8] Ben Alaya, M. and Kebaier, M. (2012). Parameter estimation for the square root diffusions: ergodic and nonergodic cases. Stochastic Models 28(4) 609-634.

[9] Ben Alaya, M. and Kebaier, M. (2013). Asymptotic behavior of the maximum likelihood estimator for ergodic and nonergodic square-root diffusions. Stochastic Analysis and Applications 31(4) 552-573.

[10] Benke, J. M. and PAP, G. (2015). Asymptotic inference for a stochastic differential equation with uniformly distributed time delay. Journal of Statistical Planning and Inference. $167182-192$.

[11] Benke, J. M. and PAP, G. (2017). One-parameter statistical model for linear stochastic differential equation with time delay. Statistics 51(3) 510-531. 
[12] Benke, J. M. and PAP, G. (2017). Local asymptotic quadraticity of statistical experiments connected with a Heston model. Acta Scientiarum Mathematicarum 83(1-2) 313344.

[13] Cox, J. C., Ingersoll, J. E. and Ross, S. A. (1985). A theory of the term structure of interest rates. Econometrica 53(2) 385-407.

[14] Diekmann, O., van Gils, S. A., Verduyn Lunel, S. M. and Walther, H.-O. (1995). Delay Equations. Functional, Complex, and Nonlinear Analysis, Springer-Verlag, New York.

[15] Erneux, T. (2009). Applied Delay Differential Equations. Springer, New York.

[16] Gushchin, A. A. and Küchler, U. (1999). Asymptotic inference for a linear stochastic differential equation with time delay. Bernoulli 5(6) 1059-1098.

[17] Gushchin, A. A. and KÜChler, U. (2000). On stationary solutions of delay differential equations driven by a Lévy process. Stochastic Processes and their Applications 88(2) $195-211$.

[18] Gushchin, A. A. and Küchler, U. (2001). Addendum to 'Asymptotic inference for a linear stochastic differential equation with time delay'. Bernoulli 7(4) 629-632.

[19] Gushchin, A. A. and KüChler, U. (2003). On parametric statistical models for stationary solutions of affine stochastic delay differential equations. Mathematical Methods of Statistics 12(1) 31-61.

[20] Gushchin, A. A. and KÜChler, U. (2011). On estimation of delay location. Statistical Inference for Stochastic Processes 14(3) 273-305.

[21] Hale, J. K. and Verduyn Lunel, S. M. (1993). Introduction to Functional Differential Equations. Springer-Verlag, New York.

[22] Heston, S. (1993). A closed-form solution for options with stochastic volatilities with applications to bond and currency options. The Review of Financial Studies 6 327-343.

[23] Höpfner, R. (2014). Asymptotic Statistics. With a View to Stochastic Processes. De Gruyter Graduate. De Gruyter, Berlin.

[24] Hurn, A. S., Lindsay, K. A. and McClelland, A. J. (2013). A quasi-maximum likelihood method for estimating the parameters of multivariate diffusions. Journal of Econometrics 172(1) 106-126.

[25] Jacod, J. and Shinyaev, A. N. (2003). Limit Theorems for Stochastic Processes, 2nd ed. Springer-Verlag, Berlin.

[26] Jeganathan, P. (1995). Some aspects of asymptotic theory with applications to time series models. Econometric Theory 11(5) 818-887. 
[27] KÜChler, U. and Kutoyants, Y. A. (2000). Delay estimation for some stationary diffusion-type processes. Scandinavian Journal of Statistics 27(3) 405-414.

[28] KÜchler, U. and Sørensen, M. (2010). A simple estimator for discrete-time samples from affine stochastic delay differential equations. Statistical Inference for Stochastic Processes 13(2) 125-132.

[29] KÜChler, U. and Sørensen, M. (2013). Statistical inference for discrete-time samples from affine stochastic delay differential equations. Bernoulli 19(2) 409-425.

[30] Kutoyants, Y. A. (2004). Statistical Inference for Ergodic Diffusion Processes. Springer Series in Statistics. Springer-Verlag London, Ltd., London.

[31] Le Cam, L. and Yang, G. L. (2000). Asymptotics in Statistics: Some Basic Concepts, 2nd ed. Springer, New York.

[32] LI, Z. and MA, C. (2015). Asymptotic properties of estimators in a stable Cox-IngersollRoss model. Stochastic Processes and their Applications 125(8) 3196-3233.

[33] Liptser, R. S. and Shiryaev, A. N. (2001). Statistics of Random Processes I. General Theory, 2nd edition. Springer-Verlag, Berlin, Heidelberg.

[34] Liz, E. amd Röst, G. (2013). Global dynamics in a commodity market model. Journal of Mathematical Analysis and Application 398(2) 707-714.

[35] MAO, X. (2007). Stochastic Differential Equations and Applications, 2nd ed. Chichester: Horwood Publishing.

[36] Overbeck, L. (1998). Estimation for continuous branching processes. Scandinavian Journal of Statistics 25(1) 111-126.

[37] Putschke, M. U. (2001). Affine stochastische Funktionaldifferentialgleichungen und lokal asymptotische Eigenschaften ihrer Parameterschätzungen (in German). PhD Thesis, Humboldt-Universität, Mathematisch-Naturwissenschaftliche Fakultät II, v, 199 S., Berlin.

[38] Reiß, M. (2002). Nonparametric Estimation for Stochastic Delay Differential Equations. PhD Thesis, Humboldt-Universität, Mathematisch-Naturwissenschaftliche Fakultät II, 123 S., Berlin.

http://dochost.rz.hu-berlin.de/dissertationen/reiss-markus-2002-02-13/

[39] Reiß, M. (2002). Minimax rates for nonparametric drift estimation in affine stochastic delay differential equations. Statistical Inference for Stochastic Processes 5(2) 131-152.

[40] Reiß, M. (2005). Adaptive estimation for affine stochastic delay differential equations. Bernoulli 11(1) 67-102. 
[41] Reiß, M., Riedle, M. and van GaAns, O. (2007). On Émery's inequality and a variationof-constants formula. Stochastic Analysis and Applications 25(2) 353-379.

[42] Revuz, D. and Yor, M. (1999). Continuous Martingales and Brownian Motion, 3rd ed. Springer-Verlag, Berlin, Heidelberg.

[43] Röst, G. and Wu, J. (2008). SEIR epidemiological model with varying infectivity and infinite delay. Mathematical Biosciences and Engineering 5(2) 389-402.

[44] Shiryaev, A. N. and Spokoiny, V. G. (2000). Statistical Experiments and Decisions. Asymptotic Theory. Advanced Series on Statistical Science \& Applied Probability, 8. World Scientific Publishing Co., Inc., River Edge, NJ.

[45] Stroock, D. W. (1993). Probability Theory, an Analytic View. Cambridge University Press, Cambridge.

[46] VAn der VAart, A. W. (1998). Asymptotic Statistics, Cambridge University Press.

[47] Wright, E. M. (1961). A functional equation in the heuristic theory of primes. The Mathematical Gazette 45 15-16.

[48] VAN ZANTEN, H. (2000). A multivariate central limit theorem for continuous local martingales. Statistics 8 Probability Letters 50(3) 229-235. 